\title{
An Investigation into the Corrosion Behaviour and Effect of Inhibitor Additions on Commercial Zn-Mg-Al Alloys
}

\author{
Lewis, Thomas A.
}

How to cite:

Lewis, Thomas A. (2018) An Investigation into the Corrosion Behaviour and Effect of Inhibitor Additions on Commercial Zn-Mg-Al Alloys. thesis, Swansea University.

http://cronfa.swan.ac.uk/Record/cronfa40713

Use policy:

This item is brought to you by Swansea University. Any person downloading material is agreeing to abide by the terms of the repository licence: copies of full text items may be used or reproduced in any format or medium, without prior permission for personal research or study, educational or non-commercial purposes only. The copyright for any work remains with the original author unless otherwise specified. The full-text must not be sold in any format or medium without the formal permission of the copyright holder. Permission for multiple reproductions should be obtained from the original author.

Authors are personally responsible for adhering to copyright and publisher restrictions when uploading content to the repository.

Please link to the metadata record in the Swansea University repository, Cronfa (link given in the citation reference above.)

http://www.swansea.ac.uk/library/researchsupport/ris-support/ 


\title{
An Investigation into the Corrosion \\ Behaviour and Effect of Inhibitor Additions \\ on Commercial Zn-Mg-Al Alloys
}

By

Thomas Adam Lewis

\author{
A Thesis \\ Presented to Swansea University \\ In fulfilment of the \\ requirements for the degree of \\ Engineering Doctorate (EngD)
}

in

Materials Engineering

College of Engineering, Swansea University, 2018

(C) Thomas A. Lewis 2018 


\section{Author Declaration}

This work has not previously been accepted in substance for any degree and is not being concurrently submitted in candidature for any degree.

Signed:

Date:

STATEMENT 1

This thesis is the result of my own investigations, except where otherwise stated. Other sources are acknowledged by footnotes giving explicit references.

Signed:

Date:

\section{STATEMENT 2}

I hereby give consent for my thesis, if accepted, to be available for photocopying and for inter-library loan, and for the title and summary to be made available to outside organisations.

Signed:

Date: 


\begin{abstract}
The general premise of this work was to better understand the corrosion behaviour of newergeneration zinc-magnesium-aluminium galvanising alloys. In addition to this, the impact of both novel and established corrosion inhibitor additions dosed into solution were studied to assess the effects to prospective alloy lifetimes and the underlying mechanisms of inhibitor action. This was through means of microstructural characterisation and analysis, and accelerated corrosion testing under immersion conditions; this included the use of the SVET, time-lapse optical microscopy, open-circuit potential, potentiodynamic polarisation, and gravimetric analysis. Accordingly, the microstructural attributes of three commercial grade zincmagnesium-aluminium alloys were studied according to the differing quantities of magnesium and aluminium included in each alloy. The primary zinc-rich dendritic phases were observed to diminish in both volume fraction percentage and in size for increasing alloying addition. This was accompanied by a corresponding increase in eutectic phase volume fraction, which consisted of a binary and ternary lamellar eutectic, as confirmed by SEM-EDS. Alongside the microstructural changes, corrosion performance was noted to improve as alloying additions were increased. This was realised by SVET-measured metal loss values, of which SVET revealed fewer anodic sites and a lessened extent of anodic evolution. Time-lapse microscopy data demonstrated that corrosion was initiated in eutectic phases, attacking the $\mathrm{MgZn}_{2}$ phase in the first instance. The improved corrosion resistance for higher alloyed samples was associated with the preferential attack of magnesium-rich phases, forming beneficial corrosion products which enabled a reduction in corrosion reaction kinetics. The remaining work utilised a selected alloy of $\mathrm{Zn}-2 \mathrm{wt} . \% \mathrm{Mg}-2 \mathrm{wt} . \% \mathrm{Al}$ composition for experimental studies. The effect of solution $\mathrm{pH}$ was next considered to understand the impact to corrosion behaviour in such environments. For neutral and alkaline conditions, a characteristic localised attack was noted, with improvements in corrosion performance corresponding to higher $\mathrm{pH}$ conditions. Acidic conditions instead led to a generalised corrosion mechanism, illustrating a more widespread and more pronounced corrosive attack on the alloy surface. The increased corrosion resistance associated with higher $\mathrm{pH}$ conditions was attributed to an enhanced presence and stability of beneficial corrosion products. Further work was performed to assess the effectiveness and mechanisms of action for both established and more novel corrosion inhibitor additions on the selected zincmagnesium-aluminium alloy. This was performed by dosing designated concentrations of the inhibitor species into solution. The addition of sodium phosphate was recognised to progressively reduce the formation and evolution of anodic sites, providing enhanced levels of corrosion resistance accordingly. The growth of anodic sites was observably restricted through
\end{abstract}


the local formation of insoluble metal phosphate precipitates, predicted to be tertiary phosphate species according to solubility calculations. An anodic inhibition effect was suggested via reaction of phosphate anions with that of metal cations in solution, to produce insoluble metal phosphate species at regions of anodic activity. An amino acid, L-tryptophan, was studied as a prospective corrosion inhibitor for the designated zinc-magnesium-aluminium alloy coated steel. The addition of this compound at higher concentrations revealed a beneficial impact to the corrosion performance, whereby metal loss values were reduced and localised anodic activity was curtailed. This was realised to transpire via the formation of a film on the sample surface, precipitating predominantly in cathodic regions and eventually extending to moderate coverage of anodic regions, according to time-lapse microscopy. The data suggested that this inhibitor species acted primarily as a cathodic inhibitor, restricting mass transport of oxygen at the sample surface. The mechanism of action was not definitively demonstrated, and several mechanisms were discussed. A rare earth metal compound in the form of cerium(III) chloride was also studied as a corrosion inhibitor for the zinc-magnesium-aluminium alloy in question. An inverse relationship between extent of corrosion and concentration of inhibitor addition was realised, whereby higher concentrations enabled favourable corrosion resistance levels. The development of anodic activity was hindered by the deposition of films at the sample surface, and these were noted to form only in regions of cathodic activity. It was proposed that these films were of a cerium oxide/hydroxide composition, and limit adsorption of oxygen at the sample surface, regulating corrosion kinetics and thus the rate of anodic growth. Accordingly, the overall data suggested that this compound acted through means of cathodic inhibition. The combination of techniques has enabled valuable insights to be gained into the corrosion behaviour of commercial zinc-magnesium-aluminium alloys in different environments; this has also aided in the understanding of the underlying mechanisms of action for a range of prospective corrosion inhibitors within the zinc-magnesium-aluminium system. 


\section{Acknowledgements}

It's difficult to know where to start, I'm thankful to so many of you. First off, I have to give a massive thank you to the whole EngD scheme at Swansea, Tata Steel Europe, and the funding bodies that have made this work possible - thank you.

I am hugely grateful to my supervisor, Jim Sullivan, not only for the patience and wisdom, but for the laughs over the years. I couldn't have asked for a better mentor to work with. This of course extends to Jon Elvins, for your character, reassurance and guidance through the years. A big thank you also to the wealth of knowledge that is the corrosion group, Materials department at Swansea, and SPECIFIC. It's been like my second home. I couldn't go without also giving my thanks to the admin family and all of those that make the whole scheme look seamless. I've caught glimpses of the behind-the-scenes work that goes on, and you're all soldiers for it. Thank you.

To friends and family, I'm so appreciative for you all. You've put up with my highs and lows, and my ever-evolving grumpy nature for these last few months. You've supported me throughout. To the hoard of friends I've made, who've come and gone over the years, thank you so much for the collective moaning, fits of laughter, and occasional bouts of intelligent conversations you've brought into my life. I'm proud to have worked with all of you fantastic people, it's been a privilege.

Ali, thank you for reminding me about most things. And that it's lunch time.

Becky, you've been there for me more than I could have ever hoped for. Thank you so much. You're my rock.

Mum, Dad, I could never articulate what you've done for me, but you've always had faith in me and encouraged me no matter what. I wouldn't be here without you.

Thank you all so much, for all that you do. I'll remember my time fondly. Apart from the polishing. 


\section{Table of Contents}

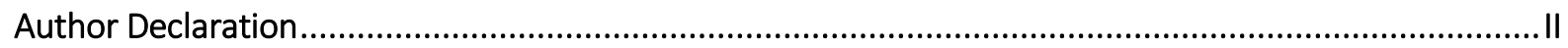

Abstract

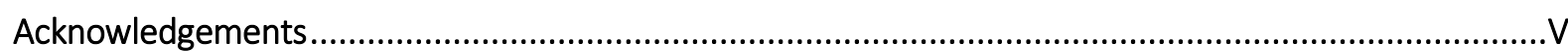

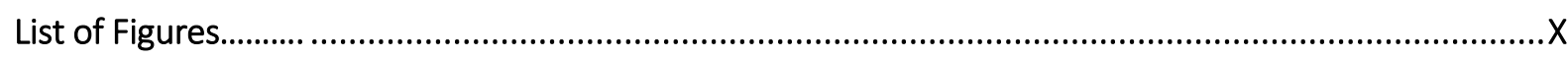

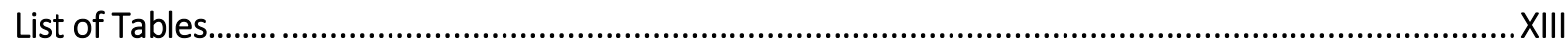

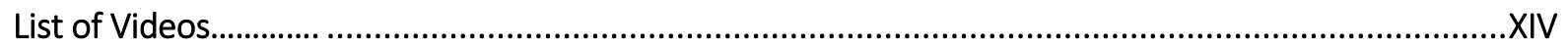

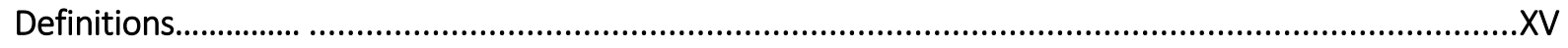

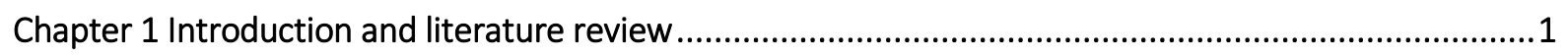

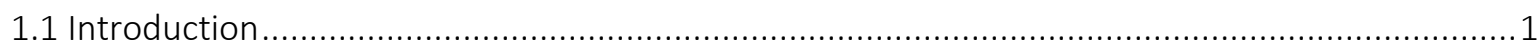

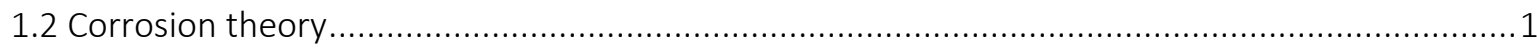

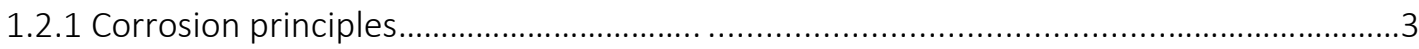

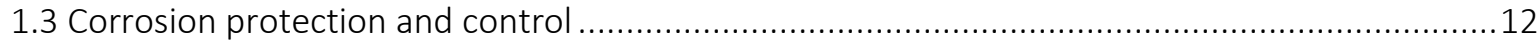

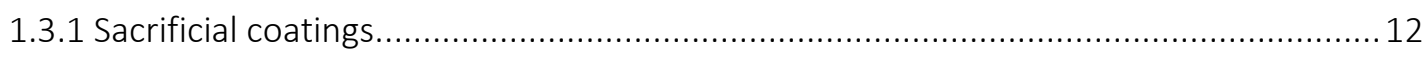

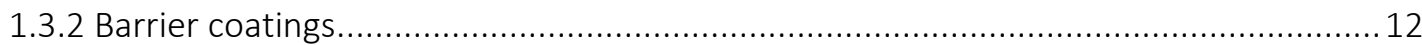

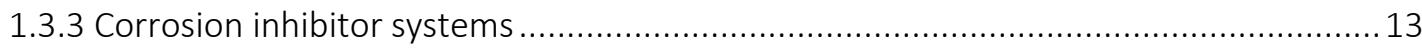

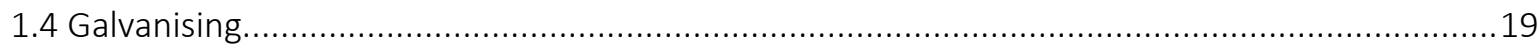

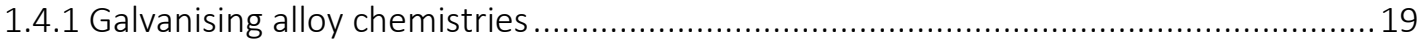

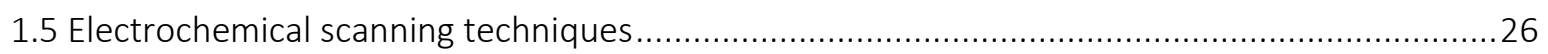

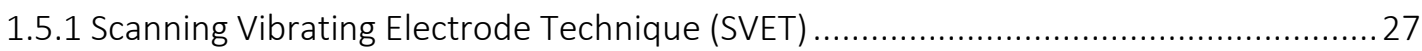

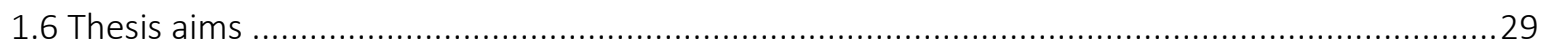

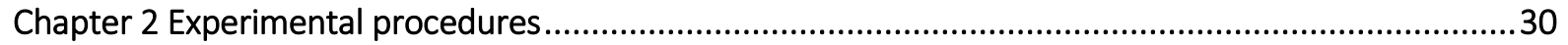

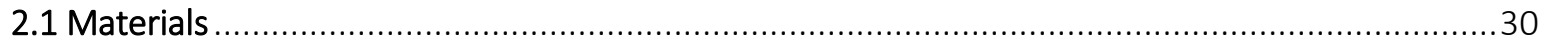

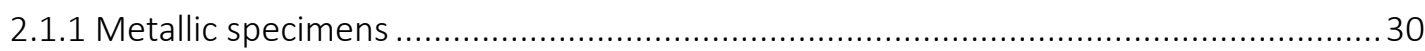

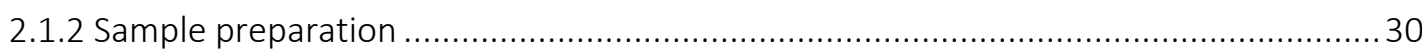

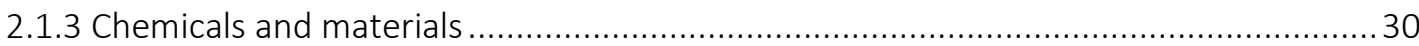

2.2 Study of corrosion behaviour using the Scanning Vibrating Electrode Technique (SVET) .............31

2.2.1 SVET apparatus and point current source response .................................................. 31

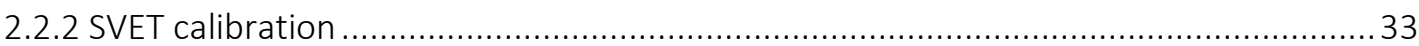

2.2.3 SVET operation for the examination of corrosion behaviour.........................................36

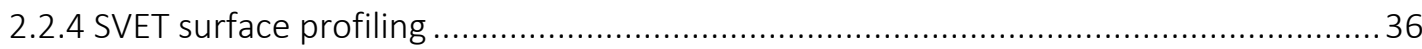

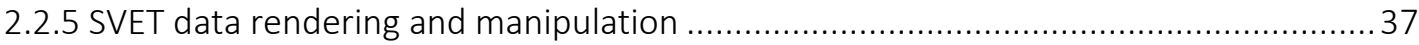

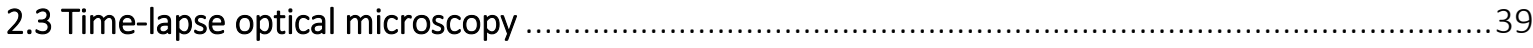

2.4 Scanning electron microscopy and energy-dispersive X-ray spectroscopy ...............................40

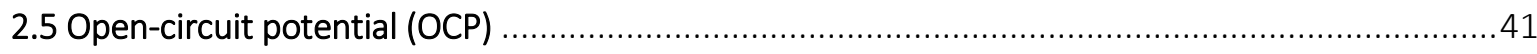

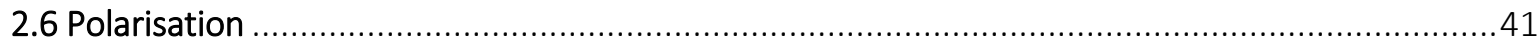

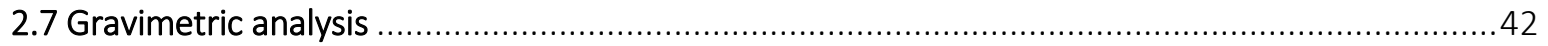


2.8.1 SVET image analysis

Chapter 3 Influence of composition variation on microstructure and corrosion behaviour of zincmagnesium-aluminium (ZMA) galvanising alloys

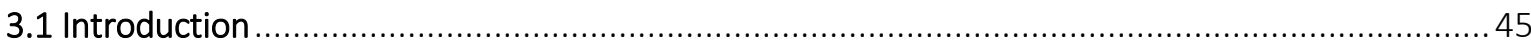

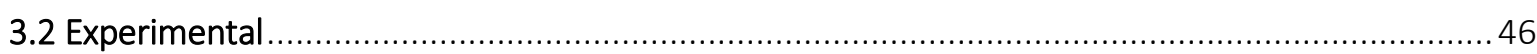

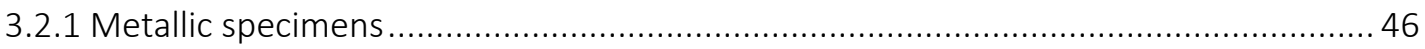

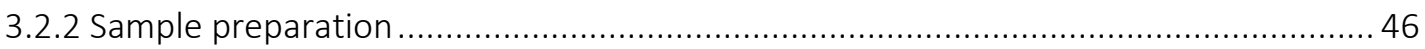

3.2.3 Assessment of ZMA alloy corrosion performance and mechanisms ......................... 47

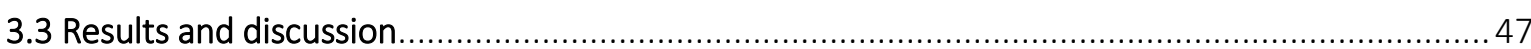

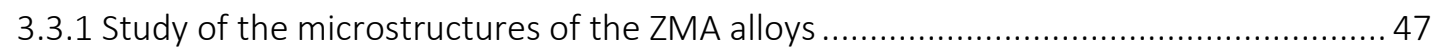

3.3.2 Investigation of corrosion behaviour using SVET ................................................... 51

3.3.3 Examination of ZMA alloy corrosion using time-lapse microscopy .............................60

3.3.4 Examination of corrosion behaviour of ZMA alloys according to alloy content variation

3.4 Conclusions

Chapter 4 The role of $\mathrm{pH}$ variation on the corrosion behaviour of a zinc-magnesium-aluminium alloy

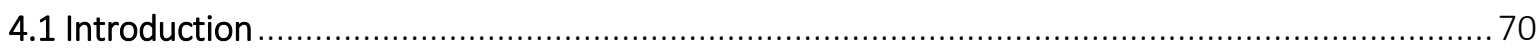

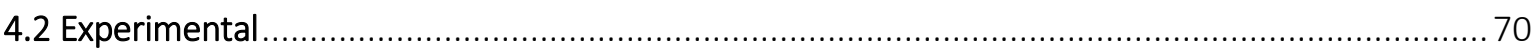

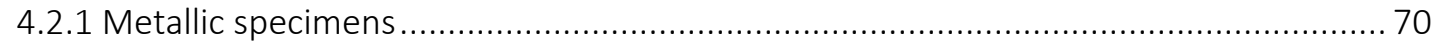

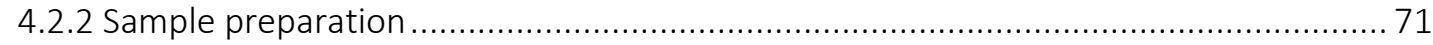

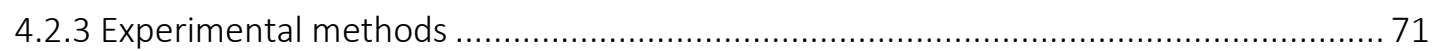

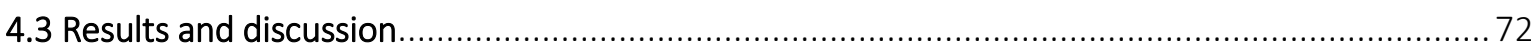

4.3.1 Assessment of $\mathrm{pH}$ variation on the corrosion behaviour of a ZMA alloy using SVET .... 72

4.3.2 Monitoring of ZMA corrosion activity using time-lapse optical microscopy................. 77

4.3.3 Electrochemical study of pH variation on ZMA 2 alloy ............................................. 79

4.3.4 Confirmation of SVET-realised metal loss using gravimetric analysis .......................... 81

4.3.5 Exploration of the effect of $\mathrm{pH}$ variation on corrosion behaviour of ZMA 2 ............... 83

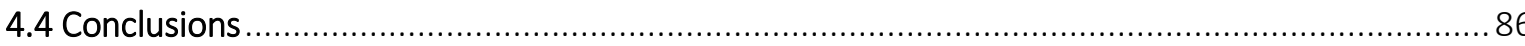

Chapter 5 The impact of dosed sodium phosphate into solution on the corrosion behaviour of a

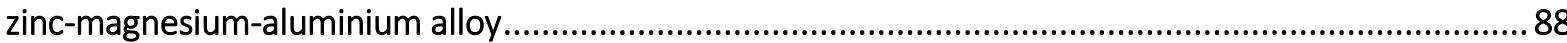

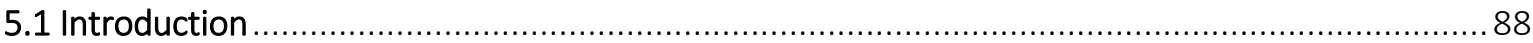

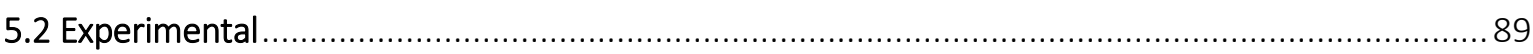

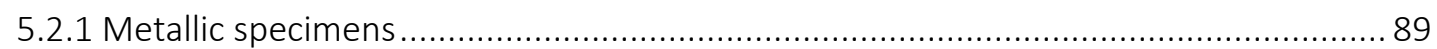

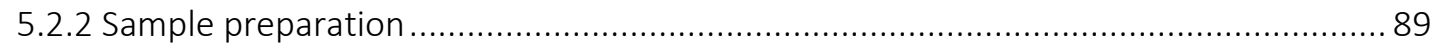

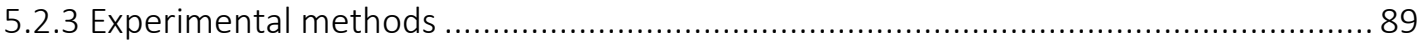

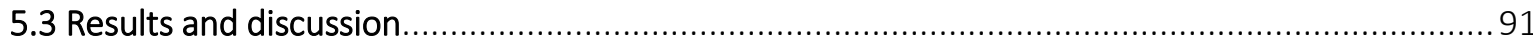


5.3.1 Study of sodium phosphate as an inhibitor on the behaviour of a ZMA alloy using SVET

5.3.2 Examination of the effects of sodium phosphate inhibitor additions on ZMA 2 using time-lapse optical microscopy.

5.3.3 Polarisation curves per the studied sodium phosphate inhibitor additions ................101

5.3.4 Surface analysis of corrosion inhibitor deposition using SEM-EDS ........................... 103

5.3.5 Calculation of solubility characteristics for the dosed sodium phosphate inhibitor ...104

5.3.6 Evaluation of sodium phosphate inhibitor action on a ZMA 2 alloy .........................106

5.4 Conclusions

Chapter 6 Study of the inhibition effects of an amino acid during corrosion of a zinc-magnesium-

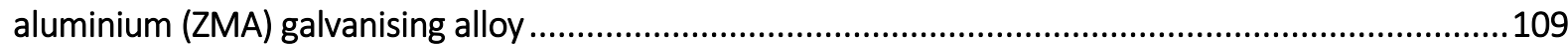

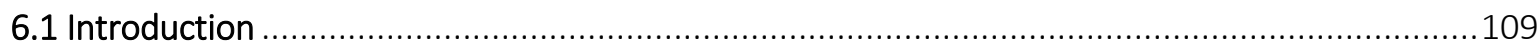

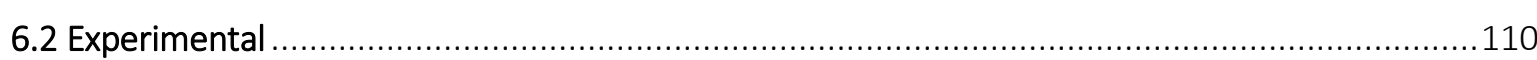

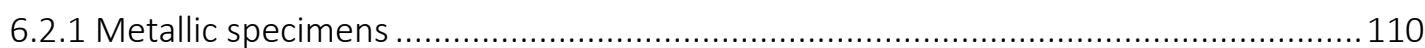

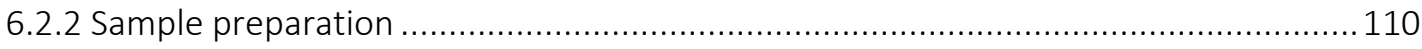

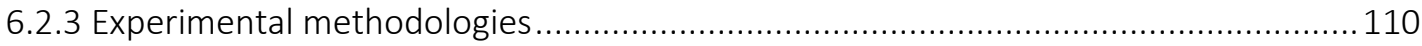

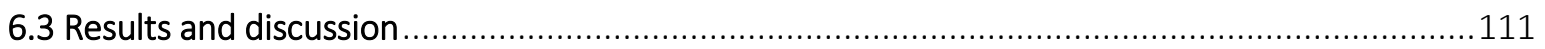

6.3.1 Determination of corrosion inhibition of a ZMA alloy by L-tryptophan using SVET ....111

6.3.2 Time-lapse optical microscopy examination of L-tryptophan interaction as a corrosion

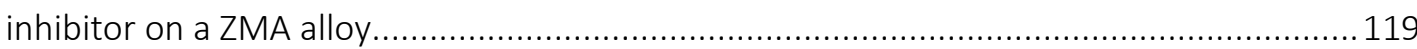

6.3.3 Open-circuit potential and potentiodynamic polarisation of varying concentrations of

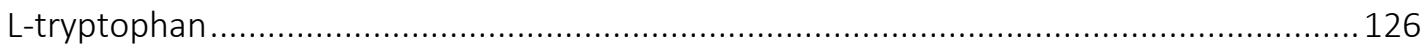

6.3.4 Surface analysis of corrosion inhibitor deposition via SEM and EDS ........................130

6.3.5 Validation of SVET-measured metal loss using gravimetric analysis .........................132

6.3.6 Evaluation of the corrosion inhibition properties of L-tryptophan in a ZMA alloy......133

6.4 Conclusions

Chapter 7 Examination of the inhibition properties of a rare earth element during corrosion of a zinc-magnesium-aluminium (ZMA) galvanising alloy...

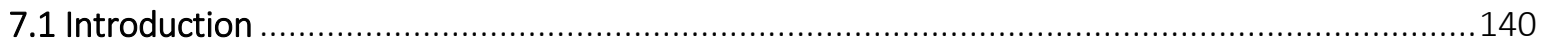

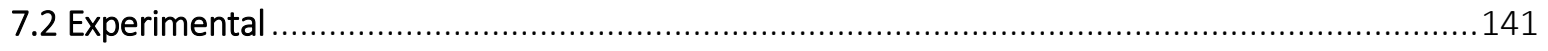

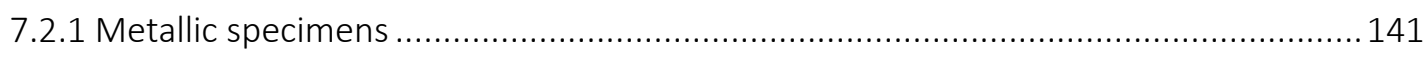

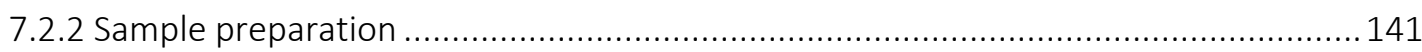

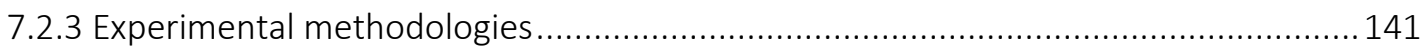

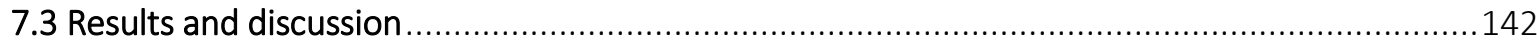

7.3.1 Corrosion inhibition effects of $\mathrm{CeCl}_{3}$ on a ZMA alloy via SVET ................................. 142

7.3.2 Examination of $\mathrm{CeCl}_{3}$ interaction as a corrosion inhibitor on a ZMA alloy using time-

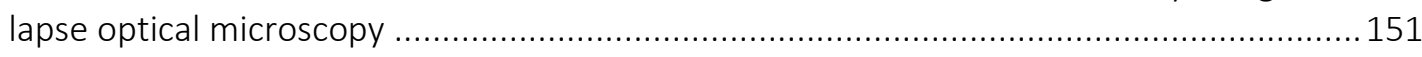

7.3.3 Open-circuit potential and potentiodynamic polarisation of $\mathrm{CeCl}_{3}$ additions............. 156

7.3.4 Study of inhibitor surface deposition using SEM and EDS ......................................160

7.3.5 Validation of SVET-measured metal loss using gravimetric analysis.........................161 
7.3.6 Exploration of the corrosion inhibition behaviour of $\mathrm{CeCl}_{3}$ in a $\mathrm{ZMA}$ alloy 163

7.4 Conclusions 167

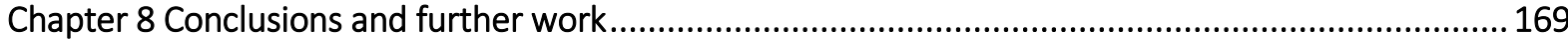

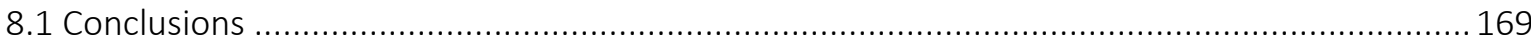

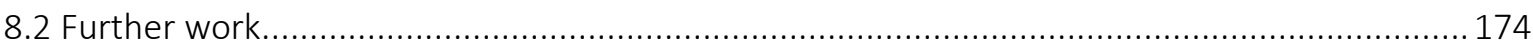

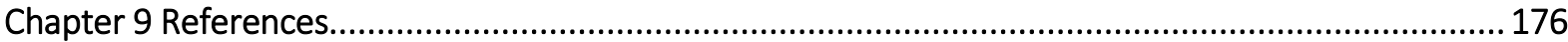

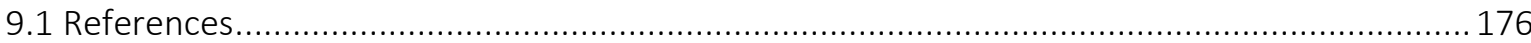

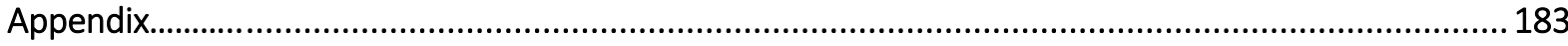




\section{List of Figures}

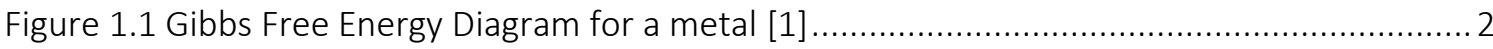

Figure 1.2 Schematic representation of typical aqueous corrosion cell as an Evans droplet ..........5

Figure 1.3 Galvanic series for metals in seawater [1] ......................................................... 6

Figure 1.4 Schematic representation of an Evans diagram for a metal immersed in an aerated

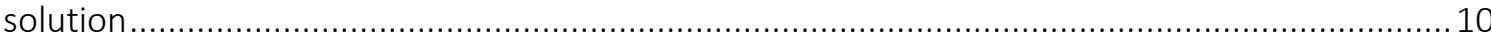

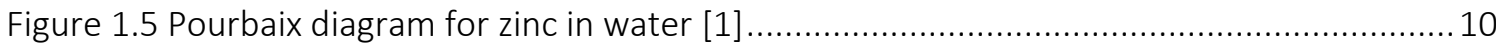

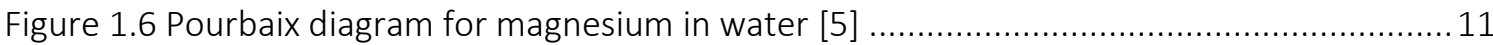

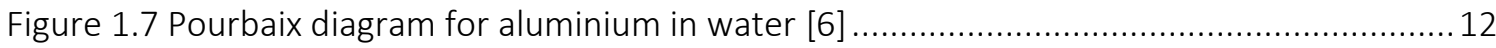

Figure 1.8 Schematic representation of the Evans diagram corresponding to anodic inhibition .. 14

Figure 1.9 Schematic representation of the Evans diagram corresponding to cathodic inhibition 15

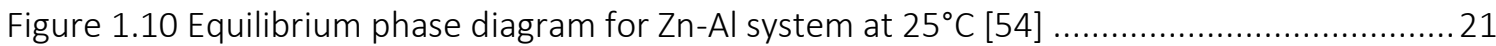

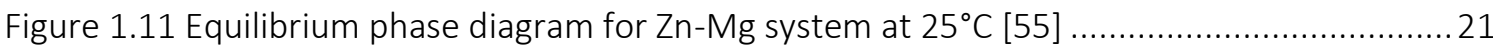

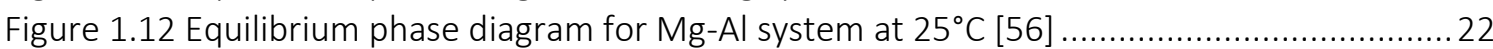

Figure 1.13 Ternary phase diagram for Zn-Mg-Al system [58] ................................................2 24

Figure 1.14 Schematic representation of typical microstructure at the cut-edge for Galfan and

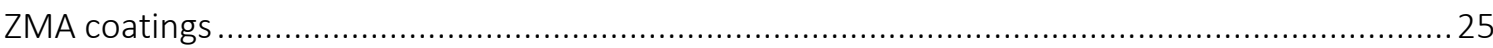

Figure 1.15 Schematic diagram of SVET operation principles above a point anodic site...............28

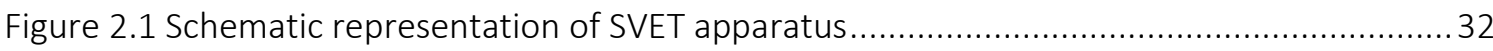

Figure 2.2 Schematic depiction of an SVET calibration setup using a two-compartment cell ........35

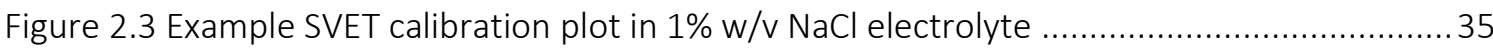

Figure 2.4 Schematic representation of time-lapse optical microscopy setup ............................. 40

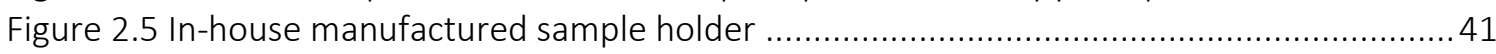

Figure 3.1 (A-C) Optical light microscope images of each ZMA alloy. All alloys encompass a

microstructure of primary dendrites and eutectic phase ............................................................ 48

Figure 3.2 Volume fraction (\%) of microstructural phases present in the three ZMA alloy

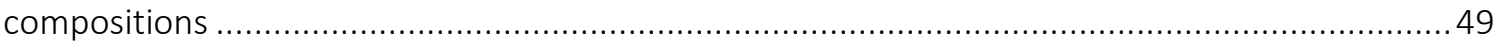

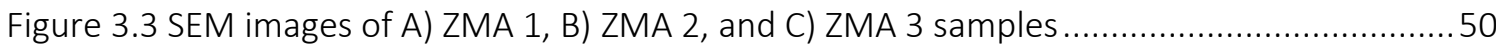

Figure 3.4 SEM-EDS analysis of: (A-D) ZMA 1, (E-H) ZMA 2, (I-L) ZMA 3 ..................................51

Figure 3.5 SVET-derived metal loss over 24-hour experiment time for the ZMA alloy range

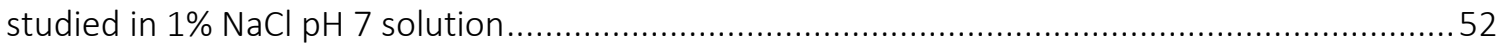

Figure 3.6 SVET-measured current density maps of ZMA 1 in $1 \% \mathrm{NaCl}$ pH 7 electrolyte ..............56

Figure 3.7 SVET-measured current density maps of ZMA 2 in $1 \% \mathrm{NaCl}$ pH 7 electrolyte ..............57

Figure 3.8 SVET-measured current density maps of ZMA 3 in $1 \% \mathrm{NaCl} p H 7$ electrolyte ..............58

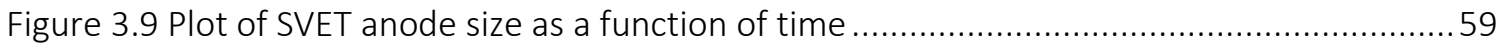

Figure 3.10 The area-averaged anodic-going current density ( $\mathrm{Ja}_{\mathrm{t}}$ ) plotted as a function of time for

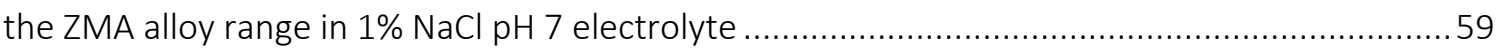

Figure 3.11 Time-lapse microscopy images taken at intervals of 2 hours in a baseline $1 \% \mathrm{NaCl}$

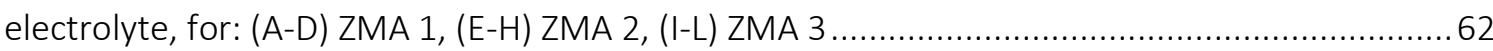

Figure 3.12 Total corroded area as measured by time-lapse microscopy for each ZMA alloy ......63 Figure 3.13 Microstructural examination of ZMA 1 using SEM, following 4 hours immersion time

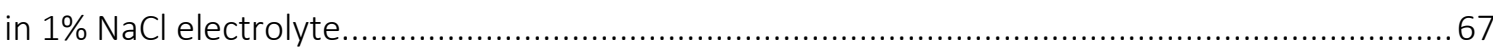

Figure 4.1 SVET estimated metal loss over 24-hour experiment time for ZMA 2 in $1 \% \mathrm{NaCl}$ at

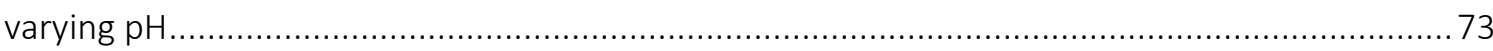

Figure 4.2 SVET-measured current density maps of ZMA 2 in $1 \% \mathrm{NaCl}$ electrolyte at A) pH 3, B) pH

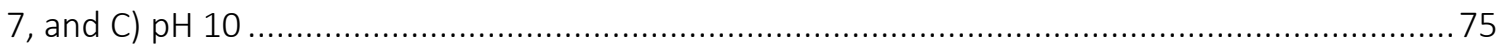

Figure 4.3 Plot of SVET anode size as a function of time for the three $\mathrm{pH}$ conditions................... 76

Figure 4.4 The area-averaged anodic-going current density $\left(\mathrm{Ja}_{\mathrm{t}}\right)$ plotted as a function of time for

ZMA 2 alloy for the studied $\mathrm{pH}$ range in $1 \% \mathrm{NaCl}$ electrolyte. 
Figure 4.5 Time-lapse microscopy images taken at intervals of 9 hours in a $1 \% \mathrm{NaCl}$ electrolyte for (A-D) pH 3, (E-H) pH 7, and (I-L) pH 10 ...

Figure 4.6 Plot of open-circuit potential testing (versus SCE) for the varied pH condition in $1 \%$

$\mathrm{NaCl}$ electrolyte

Figure 4.7 Plot of polarisation curves according to $\mathrm{pH}$ variation in a de-aerated $1 \% \mathrm{NaCl}$

electrolyte

Figure 4.8 Chart of measured metal loss from gravimetric and SVET methods including the studied $\mathrm{pH}$ range in a $1 \% \mathrm{NaCl}$ electrolyte.....

Figure 5.1 SVET estimated metal loss over 24-hour experiment time for ZMA 2 in $1 \% \mathrm{NaCl}$ pH 7 including the range of sodium phosphate inhibitor additions.

Figure 5.2 SVET-measured current density maps of ZMA 2 in $1 \% \mathrm{NaCl} \mathrm{pH} 7$ electrolyte for A) $1 \mathrm{x}$ $10^{-4} \mathrm{~mol} \mathrm{dm}^{-3}$, and B) $1 \times 10^{-3} \mathrm{~mol} \mathrm{dm}^{-3} \mathrm{Na}_{3} \mathrm{PO}_{4}$.

Figure 5.3 SVET-measured current density maps of ZMA 2 in $1 \% \mathrm{NaCl} \mathrm{pH} 7$ electrolyte for A) $1 \mathrm{x}$

$10^{-2} \mathrm{~mol} \mathrm{dm}^{-3} \mathrm{Na}_{3} \mathrm{PO}_{4}$, and B) $1 \times 10^{-4} \mathrm{~mol} \mathrm{dm}^{-3} \mathrm{Na}_{2} \mathrm{Cr}_{2} \mathrm{O}_{7}$

Figure 5.4 SVET estimated metal loss over 24-hour experiment for ZMA 2 in $1 \% \mathrm{NaCl} \mathrm{pH} \mathrm{7,}$

including sodium phosphate and sodium dichromate inhibitor additions ................................. 95

Figure 5.5 Plot of SVET anode size as a function of time for the three different sodium phosphate inhibitor species

Figure 5.6 The area-averaged anodic current density ( $\left.\mathrm{Ja}_{\mathrm{t}}\right)$ plotted as a function of time for ZMA 2

alloy in $1 \% \mathrm{NaCl} \mathrm{pH} \mathrm{7,} \mathrm{encompassing} \mathrm{the} \mathrm{range} \mathrm{of} \mathrm{studied} \mathrm{inhibitor} \mathrm{additions} \mathrm{..........................} 97$

Figure 5.7 Time-lapse microscopy images of ZMA 2 captured, in a $1 \% \mathrm{NaCl}$ pH 7 electrolyte for

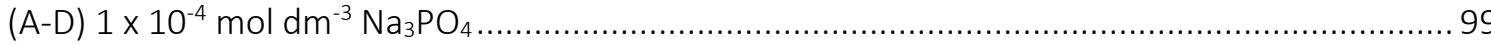

Figure 5.8 Time-lapse microscopy images of ZMA 2 captured, in a $1 \% \mathrm{NaCl}$ pH 7 electrolyte for

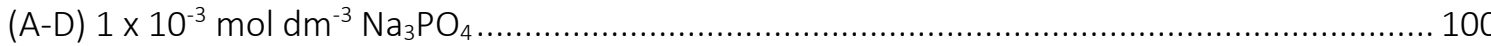

Figure 5.9 Time-lapse microscopy images of ZMA 2 captured, in a $1 \% \mathrm{NaCl}$ pH 7 electrolyte for

(A-D) $1 \times 10^{-2} \mathrm{~mol} \mathrm{dm}^{-3} \mathrm{Na}_{3} \mathrm{PO}_{4}$

101

Figure 5.10 Plot of polarisation curves for the range of dosed sodium phosphate concentrations

in $1 \% \mathrm{NaCl}$ electrolyte

Figure 5.11 (A-D) SEM and EDS analysis of a ZMA sample following immersion in $1 \times 10^{-2} \mathrm{~mol} \mathrm{dm}^{-3}$ $\mathrm{Na}_{3} \mathrm{PO}_{4}$

Figure 6.1 SVET estimated metal loss for ZMA 2 over 24 -hour experiment time in $1 \% \mathrm{NaCl} p H 7$

with given concentrations of L-tryptophan inhibitor....

Figure 6.2 SVET normal current density maps of ZMA 2 in $1 \% \mathrm{NaCl} \mathrm{pH} \mathrm{7,} \mathrm{including} 1 \times 10^{-4} \mathrm{~mol}$

$\mathrm{dm}^{-3}$ L-tryptophan

Figure 6.3 SVET normal current density maps of ZMA 2 in $1 \% \mathrm{NaCl} \mathrm{pH} \mathrm{7,} \mathrm{including} 1 \times 10^{-3} \mathrm{~mol}$

$\mathrm{dm}^{-3}$ L-tryptophan

Figure 6.4 SVET normal current density maps of ZMA 2 in $1 \% \mathrm{NaCl} \mathrm{pH} 7$, including $1 \times 10^{-2} \mathrm{~mol}$

$\mathrm{dm}^{-3}$ L-tryptophan

Figure 6.5 Plot of SVET anode size as a function of time for L-tryptophan .............................. 118

Figure 6.6 The area-averaged anodic current density $\left(\mathrm{Ja}_{\mathrm{t}}\right)$ plotted as a function of time for ZMA

alloy in $1 \% \mathrm{NaCl} \mathrm{pH} 7$ electrolyte, encompassing the range of L-tryptophan inhibitor additions 118

Figure 6.7 Time-lapse microscopy images taken at hourly intervals in a baseline $1 \% \mathrm{NaCl}$

electrolyte, including: (A-D) uninhibited control, (E-H) $1 \times 10^{-4} \mathrm{~mol} \mathrm{dm}^{-3} \operatorname{Trp},(\mathrm{I}-\mathrm{L}) 1 \times 10^{-3} \mathrm{~mol} \mathrm{dm}^{-3}$

Trp, (M-P) $1 \times 10^{-2} \mathrm{~mol} \mathrm{dm}^{-3} \operatorname{Trp}$

Figure 6.8 Size of corroded area versus time for each inhibitor concentration in $1 \% \mathrm{NaCl}$

electrolyte

Figure 6.9 Total corroded area for each inhibitor concentration in 1\% $\mathrm{NaCl}$ electrolyte ............ 125

Figure 6.10 Plot of open-circuit potential testing (versus SCE) over the range of dosed inhibitor concentrations in $1 \% \mathrm{NaCl} \mathrm{pH} 7$ baseline electrolyte.

Figure 6.11 Plot of cathodic-going polarisation curves for the range of dosed inhibitor concentrations in $1 \% \mathrm{NaCl} \mathrm{pH} 7$ baseline electrolyte. 
Figure 6.12 Plot of anodic-going polarisation curves for the range of dosed inhibitor concentrations in $1 \% \mathrm{NaCl}$ pH 7 baseline electrolyte.....

Figure 6.13 (A-G) SEM and EDS analysis of a ZMA sample following immersion in $1 \times 10^{-2} \mathrm{~mol} \mathrm{dm}^{-3}$

L-tryptophan

Figure 6.14 Chemical structure of L-tryptophan in A) zwitterion, B) protonated, and C)

deprotonated forms

Figure 7.1 SVET estimated metal loss over 24-hour experiment time for ZMA 2 in $1 \% \mathrm{NaCl} \mathrm{pH} 7$

with varying concentrations of $\mathrm{CeCl}_{3}$ inhibitor

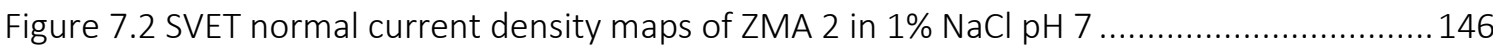

Figure 7.3 SVET normal current density maps of ZMA 2 in $1 \% \mathrm{NaCl} \mathrm{pH} 7$, including $1 \times 10^{-4} \mathrm{~mol}$

$\mathrm{dm}^{-3} \mathrm{CeCl}_{3}$

Figure 7.4 SVET normal current density maps of ZMA 2 in $1 \% \mathrm{NaCl} \mathrm{pH} \mathrm{7,} \mathrm{including} 1 \times 10^{-3} \mathrm{~mol}$

$\mathrm{dm}^{-3} \mathrm{CeCl}_{3}$

Figure 7.5 SVET normal current density maps of ZMA 2 in $1 \% \mathrm{NaCl} \mathrm{pH} \mathrm{7,} \mathrm{including} 1 \times 10^{-2} \mathrm{~mol}$

$\mathrm{dm}^{-3} \mathrm{CeCl}_{3}$

Figure 7.6 Plot of SVET anode size as a function of time for $\mathrm{CeCl}_{3}$

Figure 7.7 The area-averaged anodic-going current density $(\mathrm{Jat})$ plotted as a function of time for

ZMA 2 alloy in $1 \% \mathrm{NaCl} \mathrm{pH} 7$ electrolyte, encompassing the range of $\mathrm{CeCl}_{3}$ inhibitor additions. 150 Figure 7.8 Time-lapse microscopy images taken at hourly intervals in a baseline $1 \% \mathrm{NaCl}$

electrolyte, including: (A-D) $1 \times 10^{-4} \mathrm{~mol} \mathrm{dm}^{-3} \mathrm{CeCl}_{3},(\mathrm{E}-\mathrm{H}) 1 \times 10^{-3} \mathrm{~mol} \mathrm{dm}^{-3} \mathrm{CeCl}_{3}$, (I-L) $1 \times 10^{-2} \mathrm{~mol}$

$\mathrm{dm}^{-3} \mathrm{CeCl}_{3}$

Figure 7.9 Size of corroded area versus time for each inhibitor concentration in $1 \% \mathrm{NaCl}$

electrolyte 155

Figure 7.10 Total corroded area for each inhibitor concentration in 1\% $\mathrm{NaCl}$ electrolyte .......... 156

Figure 7.11 Plot of open-circuit potential testing (versus SCE) over the range of dosed inhibitor concentrations in $1 \% \mathrm{NaCl}$ pH 7 baseline electrolyte.....

Figure 7.12 Plot of cathodic-going polarisation curves for the range of dosed inhibitor

concentrations in $1 \% \mathrm{NaCl}$ pH 7 baseline electrolyte.... 158

Figure 7.13 Plot of anodic-going polarisation curves for the range of dosed inhibitor

concentrations in $1 \% \mathrm{NaCl}$ pH 7 baseline electrolyte.....

Figure 7.14 (A-G) SEM and EDS analysis of a ZMA sample following immersion in $1 \times 10^{-2} \mathrm{~mol} \mathrm{dm}^{-3}$

$\mathrm{CeCl}_{3}$

Figure 8.1 Summary of SVET-recorded metal loss values for the studied corrosion inhibitors ... 174

Figure 8.2 Summary of gravimetric analysis for the studied corrosion inhibitors 


\section{List of Tables}

Table 2.1 List of items used throughout body of work for experimental purposes...................... 30

Table 3.1 Composition of the three zinc-magnesium-aluminium alloy coatings of interest ......... 46

Table 4.1 Composition of the selected zinc-magnesium-aluminium alloy coating ....................... 71

Table 5.1 Composition of the selected zinc-magnesium-aluminium alloy coating ....................... 89

Table 5.2 Comparison of inhibition efficiency for sodium phosphate additions as acquired by

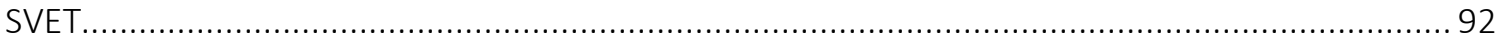

Table 5.3 Metal loss values using SVET and Tafel-extrapolation ........................................... 103

Table 5.4 Estimation of tertiary zinc phosphate precipitation likelihood in according to solution

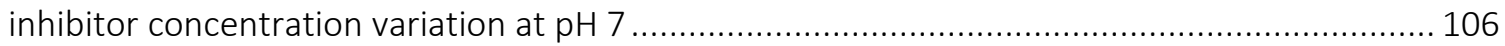

Table 5.5 Estimation of tertiary magnesium phosphate precipitation likelihood in according to

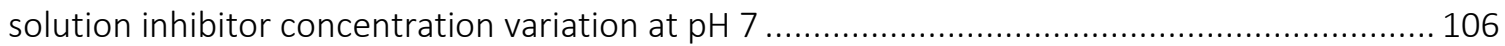

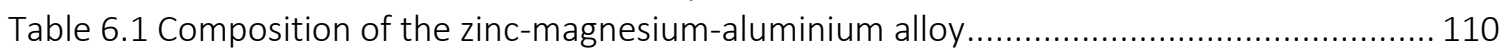

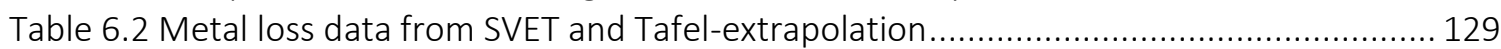

Table 6.3 Comparison of measured metal loss values via gravimetric and SVET analysis ........... 132

Table 6.4 Summary of metal loss data for different concentrations of L-tryptophan ................. 135

Table 7.1 Composition of the zinc-magnesium-aluminium alloy under scrutiny........................ 141

Table 7.2 Measured metal loss values via SVET and Tafel-extrapolation ................................... 159

Table 7.3 Comparison of measured metal loss values via gravimetric and SVET analysis ........... 162

Table 7.4 Summary of measured metal loss values................................................................ 163

Table 8.1 Summary of quantitative corrosion data for the corrosion inhibitor additions studied. 


\section{List of Videos}

Video 3.1 ZMA 1 alloy in $1 \% \mathrm{w} / \mathrm{v} \mathrm{NaCl} \mathrm{pH} 7$ electrolyte Video 3.2 ZMA 2 alloy in $1 \% \mathrm{w} / \mathrm{v} \mathrm{NaCl} \mathrm{pH} 7$ electrolyte Video 3.3 ZMA 3 alloy in $1 \% \mathrm{w} / \mathrm{v} \mathrm{NaCl} \mathrm{pH} 7$ electrolyte

Video 5.1 ZMA 2 alloy in $1 \% \mathrm{w} / \mathrm{v} \mathrm{NaCl} \mathrm{pH} 7$ with $1 \times 10^{-4} \mathrm{~mol} \mathrm{dm}^{-3}$ sodium phosphate Video $5.2 \mathrm{ZMA} 2$ alloy in $1 \% \mathrm{w} / \mathrm{v} \mathrm{NaCl} \mathrm{pH} 7$ with $1 \times 10^{-3} \mathrm{~mol} \mathrm{dm}^{-3}$ sodium phosphate Video 5.3 ZMA 2 alloy in $1 \% \mathrm{w} / \mathrm{v} \mathrm{NaCl} \mathrm{pH} 7$ with $1 \times 10^{-2} \mathrm{~mol} \mathrm{dm}^{-3}$ sodium phosphate Video $6.1 \mathrm{ZMA} 2$ alloy in $1 \% \mathrm{w} / \mathrm{v} \mathrm{NaCl} \mathrm{pH} 7$ with $1 \times 10^{-4} \mathrm{~mol} \mathrm{dm}^{-3}$ L-tryptophan Video 6.2 ZMA 2 alloy in $1 \% \mathrm{w} / \mathrm{v} \mathrm{NaCl} \mathrm{pH} 7$ with $1 \times 10^{-3} \mathrm{~mol} \mathrm{dm}^{-3}$ L-tryptophan Video 6.3 ZMA 2 alloy in $1 \% \mathrm{w} / \mathrm{v} \mathrm{NaCl} \mathrm{pH} 7$ with $1 \times 10^{-2} \mathrm{~mol} \mathrm{dm}^{-3}$ L-tryptophan Video $7.1 \mathrm{ZMA} 2$ alloy in $1 \% \mathrm{w} / \mathrm{v} \mathrm{NaCl} \mathrm{pH} 7$ with $1 \times 10^{-4} \mathrm{~mol} \mathrm{dm}^{-3}$ cerium(III) chloride Video 7.2 ZMA 2 alloy in $1 \% \mathrm{w} / \mathrm{v} \mathrm{NaCl} \mathrm{pH} 7$ with $1 \times 10^{-3} \mathrm{~mol} \mathrm{dm}^{-3}$ cerium(III) chloride Video 7.3 ZMA 2 alloy in $1 \% \mathrm{w} / \mathrm{v} \mathrm{NaCl} \mathrm{pH} 7$ with $1 \times 10^{-2} \mathrm{~mol} \mathrm{dm}^{-3}$ cerium(III) chloride 


\section{Definitions}

\begin{tabular}{|c|c|}
\hline ZMA & Zinc-magnesium-aluminium \\
\hline $\mathrm{mol} \mathrm{dm}$ & Moles per decimetre ${ }^{3}$ \\
\hline wt.(\%) & Weight percentage \\
\hline K & Degrees Kelvin \\
\hline$E_{\text {corr }}$ & Free corrosion potential \\
\hline$I_{\text {corr }}$ & Corrosion current \\
\hline$E$ & Electrode potential \\
\hline i & Current \\
\hline $\mathrm{F}$ & Faradays constant $\left(96487 \mathrm{C} \mathrm{mol}^{-1}\right)$ \\
\hline$A m^{-2}$ & Amps per metre ${ }^{2}$ \\
\hline$J_{2}$ & Normal current density \\
\hline $\mathrm{Ja}_{\mathrm{t}}$ & Area-averaged anodic current density \\
\hline tzl & Total zinc loss \\
\hline$x, y, z$ & Directional co-ordinates \\
\hline HDG & Hot-dipped galvanised steel \\
\hline LDH & Layered double hydroxides \\
\hline SVET & Scanning vibrating electrode technique \\
\hline OCP & Open-circuit potential \\
\hline SEM & Scanning electron microscopy \\
\hline EDS & Energy dispersive X-ray spectroscopy \\
\hline SCE & Saturated calomel electrode \\
\hline$K_{\mathrm{sp}}$ & Solubility product \\
\hline$Q_{s}$ & Solubility quotient \\
\hline Trp & L-tryptophan \\
\hline
\end{tabular}





\section{Chapter 1 Introduction and literature review}

\subsection{Introduction}

The use of steel and steel products has been prolific for some time and continues to be in the present day. More specifically, galvanised steels are heavily utilised, forming everyday components that are nowadays often taken for granted. Their use is ever present amongst sizeable industries, including construction and automotive sectors; these employ not only galvanised steels in their bare form, but the pre-finished steels that they also constitute. Typically, these pre-finished or galvanised steels are exposed to the environment, whereby they can perform as intended; this is by providing measurable corrosion resistance, and thus prolonging the lifetime of the product for its application of choice in a range of environments. This essentially safeguards the structure for a given period of time, comparative to that without any considered corrosion protection. Without such technology, the already ample implications associated with corrosion would be magnified. Accordingly, it is not difficult to comprehend the reason for consistent and progressive development of galvanising alloys and the technology relating to this. The intense competition within the steel industry accompanied by modern-day economic and legislative practices, both nationally and globally, add weight to the requirement of innovations in this field, acting as huge drivers in this area. Additional motivation exists in the form of EU-wide legislation, phasing out the use of hexavalent chromium $(\mathrm{Cr}(\mathrm{VI}))$ by 2019, ultimately due to the hazards it poses to the health of living organisms; this particular valence state of chromium embodies the critical component of prominent and readily used corrosion inhibitors which are responsible for the provision of excellent corrosion properties and thus, long lifetimes in coated steel systems. Measures must be taken to develop coated steel systems and pursue suitable alternatives for the less environmentally-friendly corrosion inhibitors. This pursuit opens the door for a range of research projects to consider, which ultimately forms the premise of this body of work.

\subsection{Corrosion theory}

The process of corrosion can be identified as the occurrence of an electrochemical reaction within an environment, causing degradation of a metal. This corrosion process is a large obstacle in terms of lifetimes of materials, which often leads to the eventual weakening and failure of the metal or alloy within its designated application. Obviously, this is undesirable for not only this reason, but for the tremendous costs it incurs in terms of repairs, replacements and engineering solutions that are required to overcome these inherent issues. The realisation 
of the drawbacks associated with corrosion of metals has brought about the birth of global industries devoted to preventing or minimising the onset of corrosion, or the adverse effects that they have on their associated applications. This even extends to features such as aesthetics, whereby the formation of corrosion product affects the appearance of the surface in an undesirable manner.

The reason metals corrode is the fact that the metal itself is at a higher energy state than that of its corrosion products. Metals undergo extraction methods having originated in a different state (i.e. a metal oxide) and take on some of this energy used during the extraction processes. This renders them thermodynamically unstable. The majority of metals form an oxide, producing a negative Gibbs free energy change $(\Delta G)$ to bring about a lower and more stable energy state. The activation energy barrier $\left(\Delta \mathrm{G}^{*}\right)$ is the energy needed to produce the oxides of the metal, and this $\Delta \mathrm{G} *$ value dictates the rate at which corrosion will take place, illustrated in Figure 1.1. A very slow rate of corrosion will arise from a large $\Delta G^{*}$ value, whereas a lower activation energy barrier will result in a rapid corrosion rate. The presence of water during corrosion acts to lower the $\Delta G^{*}$ value, allowing corrosion to proceed at a rapid rate.

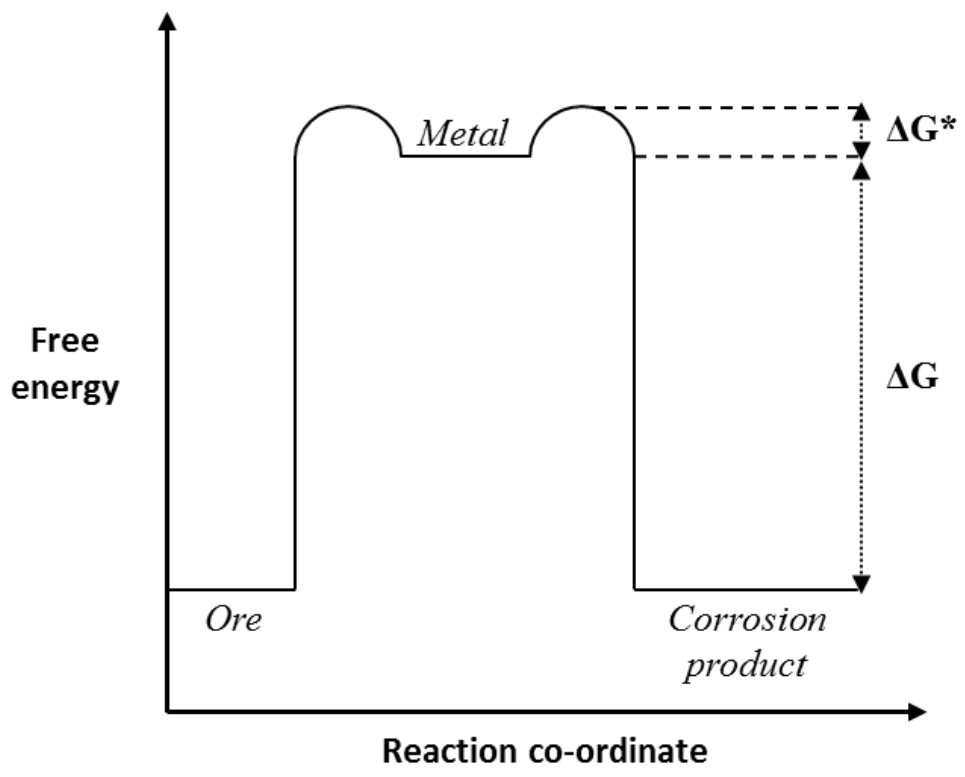

Figure 1.1 Gibbs Free Energy Diagram for a metal [1]

The $\Delta G$ value for a reaction is given by half reactions expressed as reductions:

$$
\Delta G=\Delta G_{1}-\Delta G_{2}
$$

Faradays law links the electrode potential of each half reaction to the Gibbs free energy by: 
$\mathrm{n}=$ the number of electrons transferred

$\mathrm{F}=$ charge per one mole of electrons $\left(96847 \mathrm{C} \mathrm{mol}^{-1}\right)$

$\mathrm{E}=$ the electrode potential

The standard electrode potential is a thermodynamic indicator of the ease of extraction of a particular metal, which thereby dictates how easily the metal will corrode. When a metal has a large positive electrode potential, it is more difficult to corrode whilst also being easier to extract from its ore, and the reverse also applies for a metal with a more negative electrode potential. The electrode potential of metals is measured using a standard hydrogen half-cell whereby the hydrogen is set as zero. The results are fully comparable by ensuring that solutions are kept at $1 \mathrm{~mol} \mathrm{dm}^{-3}$, gases are kept at 1 atmosphere pressure and temperature is held at 298 $K$. The standard hydrogen half-cell is made up from a sample of the metal in question, immersed into a solution of that same metals ions. The other half-cell is the platinum electrode enveloped by hydrogen gas, and these half cells are divided by a porous separator. The two electrodes are connected via a voltmeter which subsequently measures the electrode potential of the concerning pure metal, be it positive or negative.

\subsubsection{Corrosion principles}

There are two general categories of corrosion, one being dry corrosion, and the other being wet corrosion, the latter of which is common when considering metallic applications. Dry corrosion is the exposure of a metal to oxygen in the air, whereby the surface undergoes oxidation without the presence of an electrolyte. This form of corrosion is often particularly slow unless the metal is heated thereby increasing the kinetics. Wet corrosion occurs in the presence of a conducting electrolyte, where a metal is subject to anodic and cathodic activity, and electrons can flow between both sites. If any one of those mechanisms are disrupted, then corrosion will come to a halt. The combination of metal dissolution at the anode and the reduction reaction at the cathode, in the presence of an electrolyte and an electrical connection complete such a corrosion cell.

It is commonplace for both anodic and cathodic half reactions to occur on the same sample surface, and the distance between these two sites of reaction can vary. Circumstances where anodes and cathodes are separated by only a small distance and are numerous gives rise 
to generalised corrosion. Those individual sites with larger separation distances which are more easily distinguished instead form an environment of localised corrosion. The action of localised corrosion permits the ability to inflict substantial damage due to the formation of focal anodic sites on a metallic surface. The oxidation reaction typical of an anodic site results in the dissolution of metal ions originating from the metal (M) surface, and the associated emission of electrons, as given in Equation 1.3:

$$
M \leftrightharpoons M^{n+}+n e^{-}
$$

The metallic ions can either disperse into the solution or go on to react with other species, forming insoluble precipitates on the surface such as metal oxides or hydroxides. These can essentially passivate an area of the surface by impeding contact from the electrolyte and reducing the rate of corrosion, depending on the conditions. The cathodic reaction occurs in parallel with the anodic one, where electrons liberated from this anodic reaction are transported to the cathodic site via the metal itself. At normal to higher $\mathrm{pH}^{\prime} \mathrm{s}$, the oxygen reduction reaction is more common, as given in Equation 1.4:

$$
2 \mathrm{H}_{2} \mathrm{O}+\mathrm{O}_{2}+4 e^{-} \leftrightharpoons 4 \mathrm{OH}^{-}
$$

However, at lower $\mathrm{pH}$ ranges the oxygen reduction reaction becomes less favourable, and the hydrogen evolution reaction can occur in its place:

$$
2 H^{+}+2 e^{-} \leftrightharpoons H_{2}
$$

An illustration of the corrosion process under common circumstances is given in Figure 1.2: 


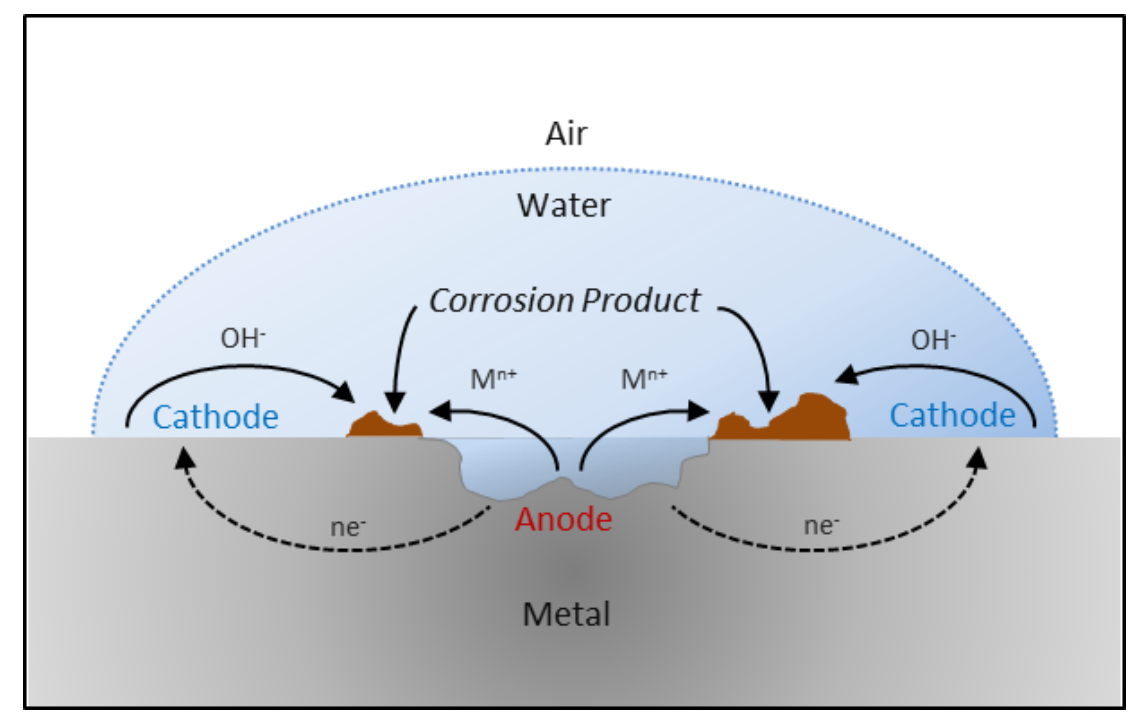

Figure 1.2 Schematic representation of typical aqueous corrosion cell as an Evans droplet

\subsubsection{Localised forms of corrosion}

There are various kinds of corrosion that can occur depending on the circumstances that arise. The nature of this work only considers specific types of corrosion, and as such, other types have been omitted from this section.

\subsubsection{Galvanic}

This form of corrosion is also well known as dissimilar metals or bimetallic corrosion. This corrosion takes place by the direct connection of two different metals in the presence of an electrolyte. This is brought about by differing electrode potentials for each of the individual metals. The standard electrode potentials, expressed as reductions, for those in an electrochemical series exhibit larger positive values per an increased level of nobility. Whereas, the opposite will be true of a metal with the most negative electrode potential. Referring to these standard electrode potentials, coupling of two different metals in a wet corrosion cell will reveal the thermodynamic likelihood that the least noble metal will act anodically, and undergo metal dissolution. Meanwhile, the other, more noble metal will normally act as the cathodic site.

Instead, considering the galvanic series given in Figure 1.3, listing the free corrosion potentials ( $\mathrm{E}_{\text {corr }}$ ) of metals in seawater immersion conditions, larger separations of measured electrode potentials indicate an increased driving force for corrosion between the coupled system. Zinc maintains a lessened degree of nobility comparative to steel; this is the reason that zinc is used as a galvanising coating for the underlying steel substrate. This then allows the zinc to corrode in preference to the steel during corrosion conditions, and accordingly, this is 
considered a form of galvanic protection. The following figure represents the galvanic series which displays the free corrosion potential for each alloy category:

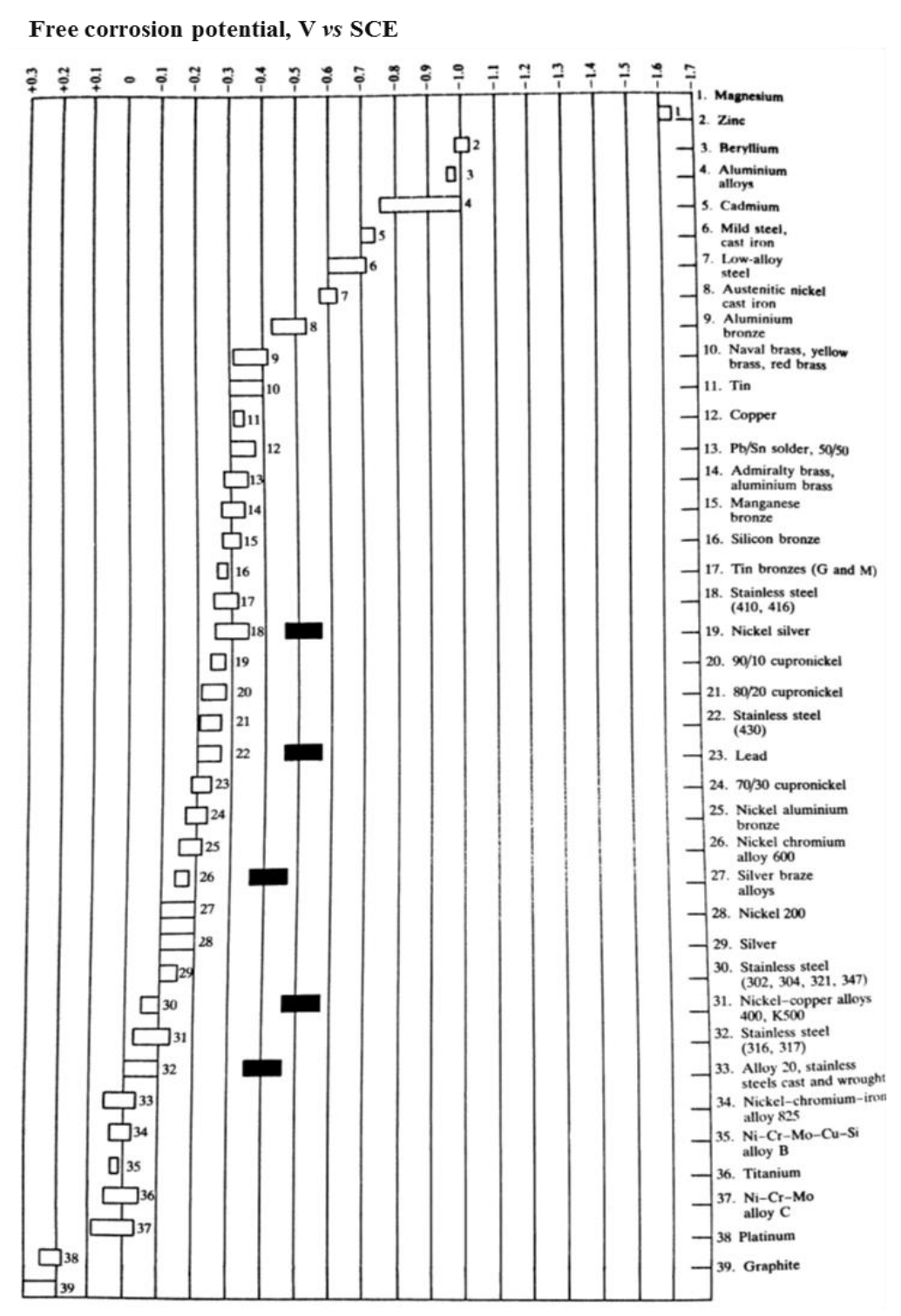

Figure 1.3 Galvanic series for metals in seawater [1]

\subsubsection{Crevice}

This form of corrosion occurs when a portion of a metal is within a confined space, or restricted environment, comparative to the remaining metal surface openly exposed to an electrolyte [2]. This can lead to conditions of focused anodic activity within the crevice, and generally these crevices would range from 25-100 $\mu \mathrm{m}$ in width. A crevice corrosion mechanism proposed for stainless steels is known as the Fontana Greene mechanism [1], although the process of crevice corrosion for other metal systems does not differ significantly.

Initially, the anodic and cathodic processes occur over the metal entirety at equal rates. As the corrosion process progresses, oxygen is depleted near the surface consequent of the 
cathodic reaction, and more oxygen must diffuse in to replace it. As oxygen is more easily replenished at the surface of the metal than within the crevice itself, the rate of oxygen reduction is diminished within the crevice. The anodic activity continues unaffected due to the increase in hydrogen ion concentration. This in essence creates a differential aeration effect whereby the depletion of oxygen within the crevice allows the formation of a focal anode. Within this crevice a net positive charge accumulates, setting up a potential difference and attracting negative ions from the electrolyte to counterbalance the charge. These negative ions are typically chloride ions and promote rates of metal dissolution, increasing corrosion further. The chloride ions can also lead to the production of acid by forming complexes with metal ions, which proceed to react with water and form acid. Corrosive attack within the crevice is consequently elevated due to the diminished $\mathrm{pH}$ conditions.

\subsubsection{Differential aeration}

The process of differential aeration corrosion is one where a metal surface is once again exposed to an electrolyte solution. The local concentration of oxygen at the surface can then act to dictate the site of preferential anodic or cathodic activity. The area with a lower oxygen concentration will predominantly form an anodic site, whereas the region with higher oxygen content will tend towards cathodic activity. This essentially generates a form of localised corrosion. Considering a droplet of rainwater upon a metallic surface, where corrosion events have initiated and proceed over the surface. The cathodic activity depletes levels of oxygen local to this site, so inward diffusion of oxygen from the atmosphere must occur via the droplet to restore these levels. The diffusion path for oxygen within the droplet varies along its hemispherical profile; a shorter diffusion path along the droplet edge will be less tortuous, whereas the centre will have the longest diffusion path. The longer diffusion path limits the oxygen availability at the central region of the metal, forming a preferential anodic site. Meanwhile, the outer edges of the droplet act as a cathodic site. Metal ions dissolved in solution diffuse outwards from the central region and meet hydroxide ions diffusing inward towards the centre, developing an intermediary ring of corrosion product. This process can be envisaged as the aqueous corrosion schematic given in Figure 1.2.

\subsubsection{Pitting}

Pitting corrosion stems from a metallurgical defect on a surface, and this can consist of a simple scratch, a dislocation, or a surface inclusion. These imperfections act as sites for preferential attack. Discounting the different initiation process of pitting corrosion, it is similar to that of crevice corrosion in that oxygen is consumed within the pit and ions are transported to achieve 
a balanced charge. Following the formation of a pit, corrosion product film coverage makes inwards oxygen diffusion more difficult. This in effect sets up a differential aeration cell and creates a stable pit. Many unstable pits can also form on the surface which are not sustained, since there is an ineffective cover of oxide film. However, some pits do survive and are recognised as metastable pits, as these are reliant on the maintenance of the oxide layer; if this oxide film is removed then the pit will expire due to oxygen diffusion. When a pit does reach a point of stabilisation and is free to propagate, the corrosion can become particularly damaging.

\subsubsection{Corrosion kinetics}

Thermodynamics can determine the eventuality of a corrosion reaction taking place, which is given in the form of electrode potential. Whilst this thermodynamic measure is highly useful, it is also limited. This is seated in the fact that it does not communicate information relating to the rate of the corrosion reaction. This rate, or the kinetics, of corrosion can be established by measuring the number of electrons that are transferred within a given period of time. Hence, corrosion kinetics can be realised via measurement of the current travelling between the anodic and the cathodic sites. As given in Equation 1.6, the Nernst equation is able to calculate the electrode potential of half cells when the concentrations and temperatures are not standard values:

$$
E=E^{O}+\frac{R T}{n F} \ln \frac{[\text { products }]}{[\text { reactants }]}
$$

$E=$ non-standard condition electrode potential

$\mathrm{E}^{0}=$ standard electrode potential (vs standard hydrogen electrode)

$\mathrm{R}=$ molar gas constant

$\mathrm{T}=$ temperature $(\mathrm{K})$

[products] $=$ product concentration (oxidation)

[reactants] $=$ reactant concentration (reduction)

Going on to consider corrosion events on a single metallic surface, where anodic and cathodic reaction rates are given by currents $l_{a}$ and $I_{c}$, respectively. As given in Equation 1.7, when the anodic and cathodic reactions are occurring at equal rates then the metal is in a state of equilibrium and no current is flowing; this is the equivalent of the exchange current density $\left(I_{0}\right)$. 


$$
I_{a}=-I_{c}=I_{0}
$$

However, when a metal is in the process of corroding then this equilibrium is lost and the metal has a potential that differs from that predicted by the Nernst equation. These metals are therefore said to be polarised in relation to the equilibrium potential by a polarisation or overpotential, $\eta[3]$.

$$
\eta=E-E_{e q}
$$

$\mathrm{E}=$ electrode potential

$E_{\text {eq }}=$ Nernst predicted potential (zero current)

These over-potentials can then be incorporated for construction of Tafel data.

\subsubsection{Evans diagram}

A single Evans diagram summarises current and potential data by utilising Tafel data for each electrode reaction. It illustrates the potential that a freely corroding system adopts $\left(E_{c o r r}\right)$, as well as the rate at which it will corrode in the form of corrosion current ( $\left.I_{\text {corr }}\right)$. The Tafel data is taken from a Tafel plot, which is a linear semi-log plot, of log I against $\eta$ for the relative oxidation and reduction half reactions. The Tafel plot characterises the effects of $\eta$ on each half reaction taking place at an electrode. This type of plot can convey valuable information relating to the rate of reaction for overall corrosion events in a system. Upon construction of an Evans diagram, a plot where rates of metal oxidation and oxygen reduction taking place are balanced, a stable corresponding value of potential can be realised. When both the anodic and cathodic processes are occurring at an equal rate and the corresponding lines overlap one another on the diagram, both $I_{\text {corr }}$ and $E_{\text {corr }}$ values can be identified. 


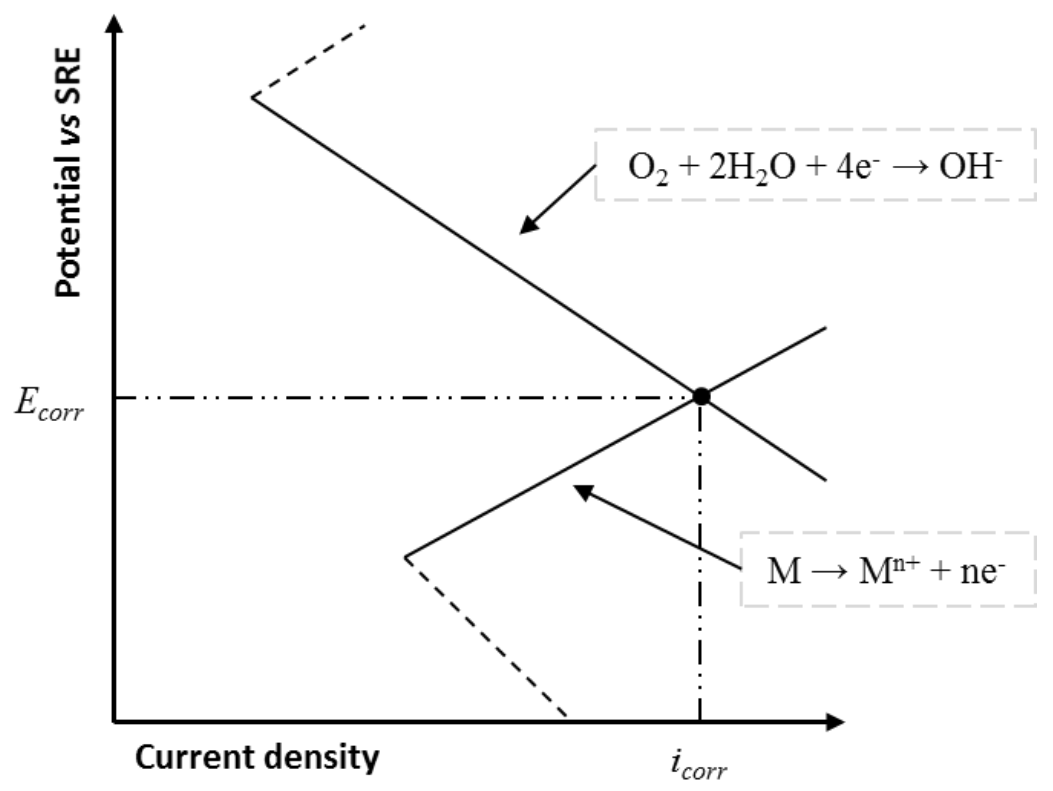

Figure 1.4 Schematic representation of an Evans diagram for a metal immersed in an aerated solution

\subsubsection{Pourbaix diagram}

Marcel Pourbaix was an electrochemist whom devised single diagrams that summarise complex corrosion systems for particular metals. The Pourbaix diagram displays whether a metal system is in a state of corrosion, immunity, or passivity depending on the conditions of potential and $\mathrm{pH}[4]$.

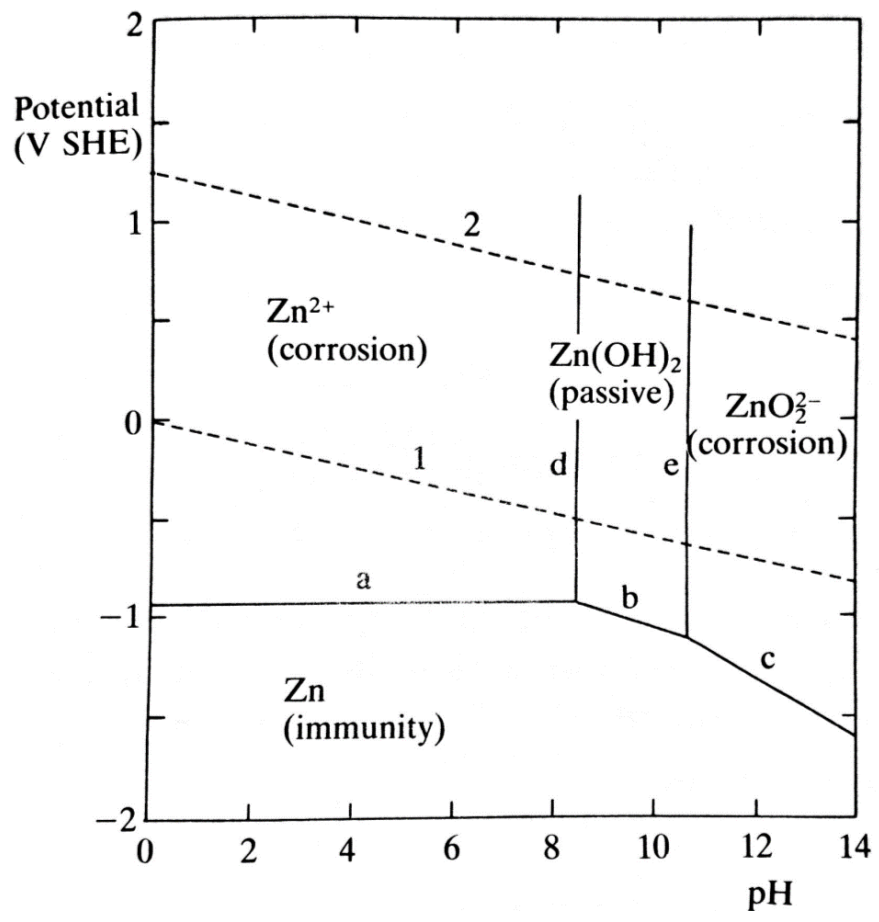

Figure 1.5 Pourbaix diagram for zinc in water [1] 
The reaction state is given according to a plot of the potential and the $\mathrm{pH}$, and three different states were outlined by Pourbaix. A metal is recognised to be actively corroding when the concentration of metal ions is $\geq 1 \times 10^{-6} \mathrm{~mol} \mathrm{dm}^{-3}$, accordant with the continuing destruction of the metal. If the metal ion concentration is below the given value of $1 \times 10^{-6} \mathrm{~mol} \mathrm{dm}^{-3}$ then it is understood to be in a state of immunity, whereby corrosion is understood to be thermodynamically impossible in the given environment. Alternatively, the metal is seen to be in a passive state and corrosion is prevented by surface modification, such as protection by an insoluble layer (i.e. a metal hydroxide film). The Pourbaix diagram enables otherwise complex systems to be interpreted more easily regarding the potential and $\mathrm{pH}$ environment.

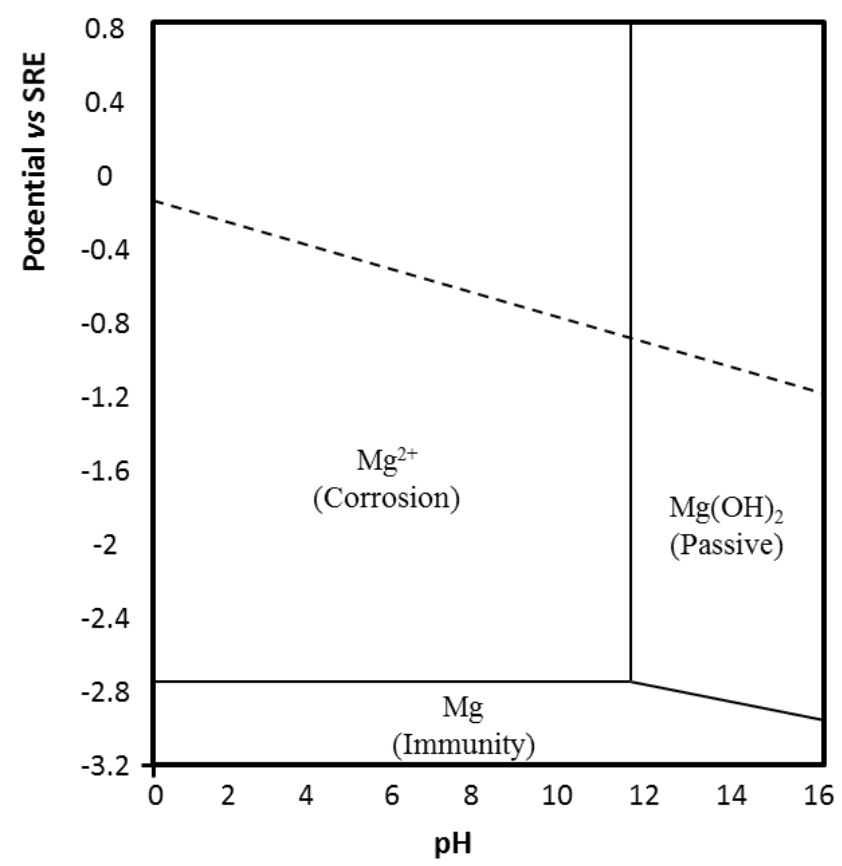

Figure 1.6 Pourbaix diagram for magnesium in water [5] 


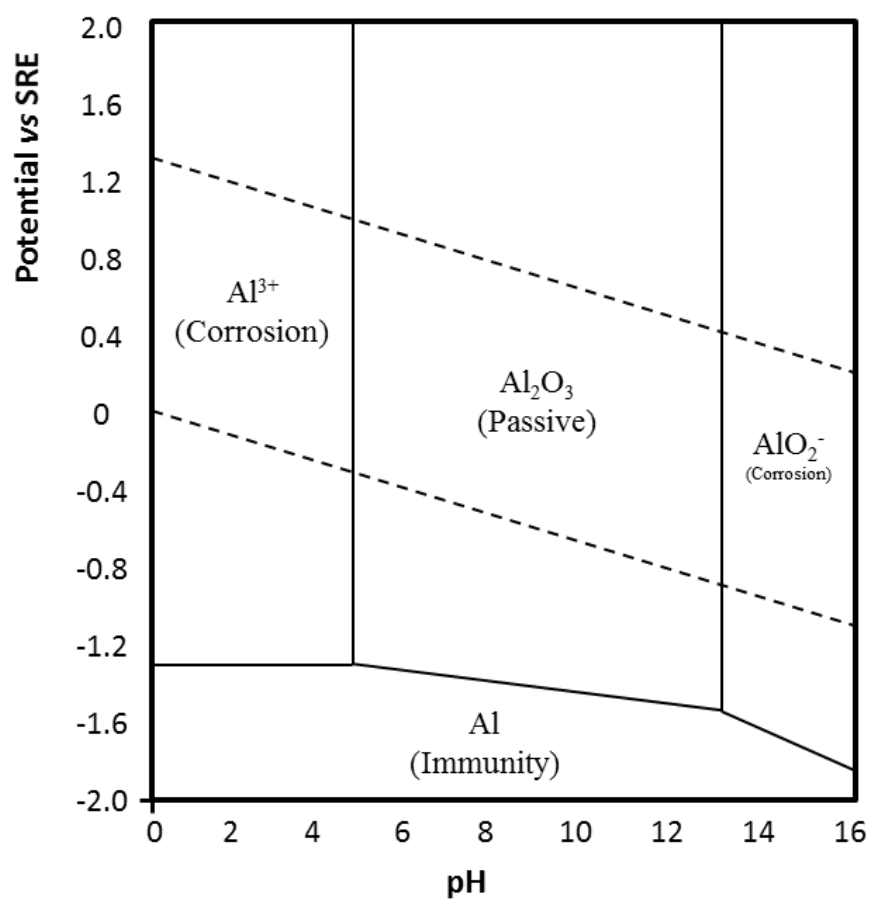

Figure 1.7 Pourbaix diagram for aluminium in water [6]

\subsection{Corrosion protection and control}

There are measures designed to diminish the rate at which corrosion occurs or prevent the onset of corrosion for as long as is feasible. This can be accomplished by eliminating one of the four elements to an electrochemical cell, consisting of the anode, the cathode, the electrolyte solution, or the electrical contact established between both anodic and cathodic features.

\subsubsection{Sacrificial coatings}

Another method of corrosion prevention is the application of a coating which corrodes in preference to that metal which is being protected. This follows the principle of dissimilar metals corrosion whereby a metal with a more negative electrode potential will act as a site for anodic activity. Therefore, the anodic metal will undergo preferential attack, thereby cathodically protecting the normally susceptible metal species. A commonly employed instance of this is the use of zinc to protect a steel base substrate.

\subsubsection{Barrier coatings}

Another simple method for corrosion prevention is via the physical separation of the exposed metal by a barrier coating. This barrier removes direct contact of the material to the electrolyte, excluding direct contact with ionic species, and so reducing the rate of the corrosion process. However, any defects or scratches that create a break in this coating will trigger the onset of corrosion once more, typically leading to delamination of the coating. Examples of barrier 
coatings are organic coatings for construction purposes, or simply the application of paint. It is worth noting that an organic coating of this nature will still allow oxygen and water to permeate, reaching the underlying substrate. As useful as this method of protection is, it is not permanent, and corrosion will eventually occur.

\subsubsection{Corrosion inhibitor systems}

Corrosion inhibitors are additives included into the pre-treatment or primer layers of organic coating systems that function to slow down the overall corrosion rate of a system. Corrosion inhibitor additions can be added in minute quantities whilst producing hefty improvements in levels of corrosion resistance. These additions act to affect either one half, or both half reactions associated with corrosion events, limiting the rate at which corrosion progresses.

\subsubsection{Anodic inhibitors}

Anodic inhibitor species act to control the metal dissolution reaction and can form lowsolubility precipitates by reacting with metal ions produced during corrosion. The precipitates that form plug the anodic site and reduce the anodic rate of corrosion, lowering the corrosion current with respect to the Evans diagram, as given in Figure 1.8. Examples of inhibitor species used can be phosphates $\left(\mathrm{PO}_{4}{ }^{3-}\right)$, molybdates $\left(\mathrm{MoO}_{4}{ }^{2-}\right)$ and chromates $\left(\mathrm{CrO}_{4}{ }^{2-}\right)$. When dealing with organically-coated steel systems, metal salt inhibitors are commonly implemented due to their tendencies for low solubility levels. A consequence of the rise in $E_{\text {corr }}$ from the presence of an anodic inhibitor is that in the instance where an insufficient quantity is present, aggressive pitting corrosion can occur. 


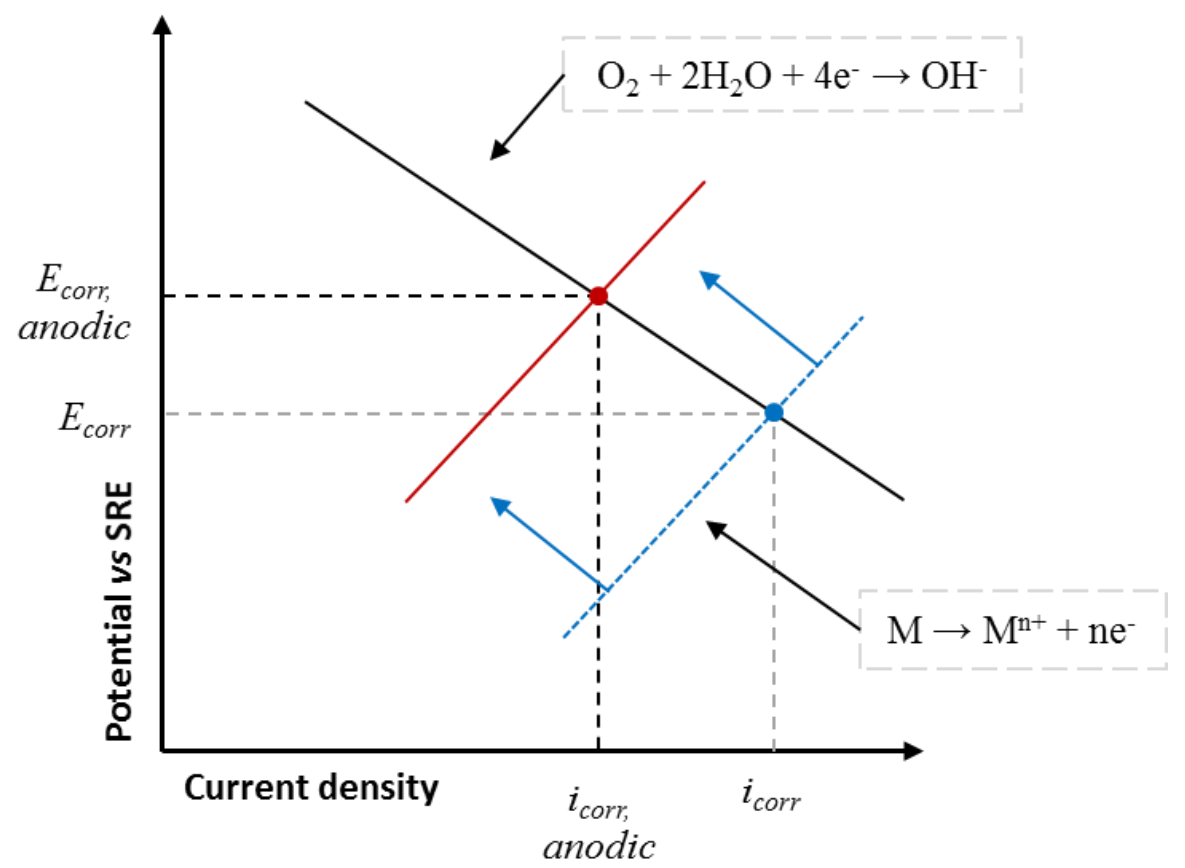

Figure 1.8 Schematic representation of the Evans diagram corresponding to anodic inhibition

\subsubsection{Cathodic inhibitors}

Cathodic inhibitors work in a similar manner to anodic inhibitors, in as much as they can precipitate insoluble layers, but at the site of cathodic activity instead. The employment of cathodic inhibition offers the benefit of lowering not only the corrosion current, but the free corrosion potential as well, as visualised in Figure 1.9. It is typical for the additions to eventually form oxides or hydroxides at the site of the cathode, limiting the availability of oxygen reaching the surface and ultimately, slowing the rate of corrosion. Whilst cathodic inhibitors are recognised as an effective means of limiting the extent of corrosive attack on a system, they are not considered as effective as anodic inhibitors; this is because they do not intercept the most aggressive half of the reaction. Yet, they are advantageous in that they do not raise $E_{\text {corr }}$ and thus, are a safer potential option. Despite this, they are still less effective than anodic inhibition and can also bring about hydrogen embrittlement. 


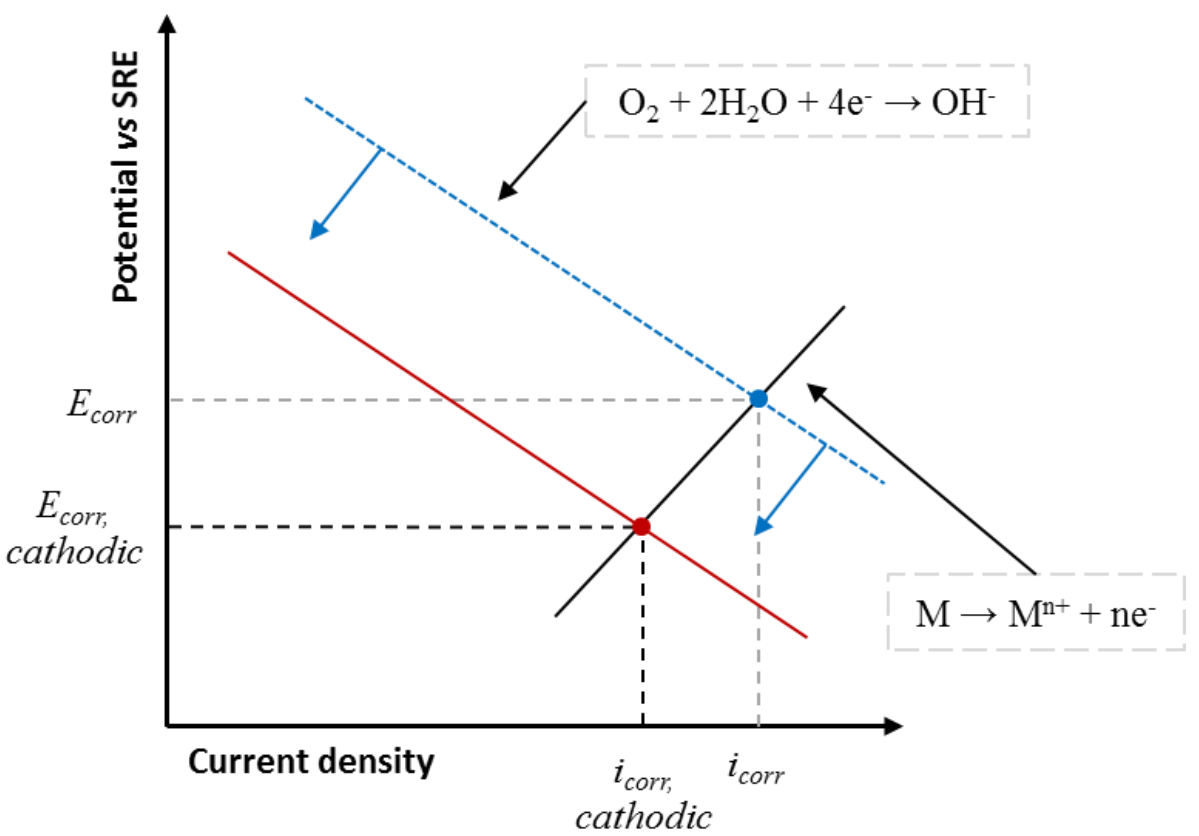

Figure 1.9 Schematic representation of the Evans diagram corresponding to cathodic inhibition

\subsubsection{Chromate}

Chromate is one of the more significant corrosion inhibitors understood to grant large improvements in corrosion performance, and has been incorporated into galvanised steel products by industry for many years. Contrary to its effectiveness, it has also proven to be highly hazardous to health and within living organisms. For this reason, within the EU it is included as part of REACH legislation and consequently its use is being phased out for such applications. This forms the immense driver for industry, both within the EU and worldwide, to seek alternatives for this compound; any suitable alternative must be able to provide similar levels of corrosion protection to ensure product lifetimes, and in that same vein, minimise costs.

Chromate is often utilised as a conversion coating but can also be used as a soluble corrosion inhibitor within a coating. The benefit of chromate is that when used as a conversion coating, it is 'self-healing', diffusing through solution and slowing pit growth, which is a difficult property to find from alternatives [7], [8]. It is understood that under neutral pH conditions, $\mathrm{Cr}(\mathrm{VI})$ readily reduces to $\mathrm{Cr}(\mathrm{III})$ to form a chromium oxide layer.

$$
2 \mathrm{CrO}_{4}^{2-}+5 \mathrm{H}_{2} \mathrm{O}+6 e^{-} \rightarrow \mathrm{Cr}_{2} \mathrm{O}_{3}+10 \mathrm{OH}^{-}
$$

This reduction reaction will often occur at sites of oxidation (at the anode), permitting the observable healing properties. The conversion coating on the steel surface often still contains 
$\mathrm{Cr}(\mathrm{VI})$, present in solution, which then reduce to the trivalent form and passivate the surface at anodic sites [9]-[11]. A mixed salt of zinc chromate, potassium chromate and zinc hydroxide form the basis for a commonly employed pigment known as zinc yellow. A consequence of the zinc hydroxide addition within this pigment is the elevation in $\mathrm{pH}$, favouring $\mathrm{Cr}$ (III) stability and encouraging formation of protective $\mathrm{Zn}(\mathrm{OH})_{2}$ at cathodic sites [9], [12].

\subsubsection{Associated hazards of chromate}

Chromium in its hexavalent state is understood to be acutely damaging when interacting with cellular tissue, causing skin ulceration, kidney and lung impairment, and ultimately, DNA damage [13], [14]. A range of research has been conducted proving that $\mathrm{Cr}(\mathrm{VI})$ is a genotoxic compound, and also possesses mutagenic and carcinogenic properties [14]-[19]. It is stated that the oxidation state of chromium-based compounds is key, and the aforementioned conditions do not occur when instead considering $\mathrm{Cr}(\mathrm{III})$ [13].

\subsubsection{Phosphate}

Phosphates are considered to be a viable alternative to chromates, and their use in place of chromates is widespread within industry. Phosphates are thought to be anodic inhibitors, offering appreciable corrosion inhibition in the presence of oxygen [20], [21]. Zin et al. explained that specific environmental conditions can enable phosphates to act as cathodic inhibitors; this arises via phosphate anions in solution reacting with metal cations from the anodic site, enabling precipitation of a surface film. This cathodic inhibition quality has been observed using polyphosphates and orthophosphates containing divalent $\mathrm{Ca}^{2+}$ or $\mathrm{Zn}^{2+}$ ions in solution, restricting access of dissolved oxygen to the metal surface [20], [22], [23].

With regard to application, phosphate has been utilised for some time, not only as a soluble corrosion inhibitor but also as a conversion coating [11]. Furthermore, phosphate has been incorporated into paint formulations as a pigment to improve corrosion resistance levels [20]. Typically, zinc phosphate is used for such applications, due to its level of performance more akin to the zinc yellow chromate-based formulation [11]. Other studies into phosphate on zinc and its alloys demonstrated a greater extent of corrosion inhibition in lower $\mathrm{pH}$ environments [20]. The work conducted by Bastos et al. did highlight that in a chloridecontaining environment, the action of phosphate took multiple hours to provide sufficient surface protection, in comparison with chromate which was nearly instantaneous. This was also observed on an iron surface, where very minimal corrosion inhibition effects were perceivable, especially in the early stages of immersion [9]. Considering this same iron surface, open circuit potential measurements revealed that when zinc phosphate additions were introduced into 
solution, there was little shift in potential observed comparative to the uninhibited solution. However, Bastos et al. showed that chromate-containing zinc yellow contrarily revealed a rapid increase in potential.

Considering the interaction of sodium phosphate in solution on a galvanised steel, it is understood that zinc ions released at an anodic site react with the phosphate, forming a zinc phosphate precipitate:

$$
3 \mathrm{Zn}^{2+}+2\left(\mathrm{PO}_{4}\right)^{3-} \rightarrow \mathrm{Zn}_{3}\left(\mathrm{PO}_{4}\right)_{2}
$$

Only a minute concentration of $\mathrm{Zn}^{2+}$ ions are necessary for precipitation of zinc phosphate due to its low solubility limit [24]. This indicates how zinc phosphate can form in preference to zinc hydroxide or zinc carbonate species.

It is worth noting that in some parts of the world, phosphates are recognised as nonhazardous to human health, as well as acceptable from an environmental perspective [25]. Contrarily, environmental regulations also consider them toxic to aquatic life and so the extent of their use is restricted by law [26]. However, due to the widespread industrial application of phosphate for means of conversion coatings and corrosion inhibition, sodium phosphate has been selected as one of the corrosion inhibitors under investigation within this body of work.

\subsubsection{Inhibitor systems}

Having discussed some powerful corrosion inhibitors above, there are a range of different compounds utilised for purposes of corrosion inhibition, acting on the anodic or cathodic reaction, or both. Again, these compounds can be used as sparingly soluble inhibitor pigments incorporated into organic coatings, as surface pre-treatments, and as conversion coatings to promote coating adhesion. Examples of these are silicates, molybdates, borates, phosphonates, tungstates, and even rare-earth metals. Silicates have been shown to match the inhibition capability of phosphates under polarisation conditions [21]. However, that same study recognised that molybdate, tetraborate, and phosphonates exhibited lower degrees of effectiveness than headline inhibitors such as chromates. A similar result was observed for molybdate and tungstate additions compared to chromate [27]. Furthermore, molybdate was shown to be an effective self-healing coating when used in conjunction with an iron surface [28]. Powell et al. went on to show that individual rare-earth metal cation additions (cerium, lanthanum, yttrium) to an aqueous chloride electrolyte acted as cathodic inhibitors and were 
highly effective; these also benefitted from no localised corrosion features induced as a result of low concentrations, often observed in some anodic inhibitor systems [27].

\subsubsection{Organic inhibitors}

The continuing focus on phasing out chromate-based compounds and instead developing corrosion inhibitors with environmentally-friendly properties has brought about research into compounds such as amino acids [29]-[32] or other organic inhibitors [33]. Bereket et al. conducted an investigation into the effect of amino acids and hydroxy carboxylic acids in chloride-containing solutions on an aluminium alloy [34]. It was found during polarisation experiments that both types of inhibitor exhibited high degrees of inhibitor efficiency capable of competing with chromates with regard to pitting. Bereket et al. found that amino acids were more effective in acidic environments [34], whereas this was true for hydroxy carboxylic acids in neutral or alkaline environments. Additionally, it was suggested that amino acids have a greater inhibition effect when increasingly distant from the $\mathrm{pH}$ value seated around their isoelectric point, primarily a result of higher dipole moments [34]. A study into the inhibition effect of amino acids on steel in acidic media showed that they were indeed effective according to polarisation measurements and relevant data extraction [35]. They were realised to inhibit corrosion via surface adsorption, and the extent of inhibition was dependant on the individual amino acid and the concentration in which it was used. Several bodies of work have been completed exploring the effects of numerous amino acids for the corrosion of steels in acidic environments [36]-[38]. The inhibition efficiencies offered by these varied according to species concentration, solution temperature, and the molecular structure, all of which formed a film adsorbed to the substrate surface. It was noted that much of the work reviewed on amino acid inhibitor additions was conducted under perturbed conditions, rather than that of freely corroding conditions, thereby potentially affecting the results obtained.

\subsubsection{Cerium salts}

Lanthanide salts are understood to be useful prospective alternatives to chromate-based compounds as corrosion inhibitors, due to their ability to form insoluble hydroxide layers and act as cathodic inhibitors [39]. These are considered to be rare-earth elements, but unlike the implication, not all of the elements classified as lanthanides are in fact limited in terms of abundance; instead, the likes of cerium are akin to copper in terms of presence in the earth's crust [39]. Bethencourt et al. also presented the point that these lanthanide compounds maintain a low degree of toxicity and are not considered as hazardous to human health. This is highly relevant due to the consideration of these compounds as potential chromate 
replacements. Research conducted by several authors deduced that cerium salts impacted on the cathodic behaviour of a range of metals, inhibiting the cathodic reaction via the formation of an insoluble film [40]-[43]. Literature has reported that in these cathodic areas where oxygen reduction occurs, cerium-based oxides and hydroxides form [43]-[46]. The reported valence of the cerium-rich layers varies across literature [41], although it has been suggested that this variation is in part, a consequence of differing lengths of immersion time in the cerium salt [44], [45]. An optimum efficiency in terms of inhibition has not been strictly linked with a particular concentration, as this depends on the metal or alloy in question [39]. However, cerium additions have been shown as highly effective [27], [45], and consequently offer great potential.

\subsection{Galvanising}

Galvanising is a form of zinc, or zinc alloy coating that is applied to a steel substrate as a method of providing protection to the underlying steel. The most prevalent method of application is via hot dip galvanising; this is where the substrate metal, such as strip steel, is immersed into a bath of molten zinc or zinc alloy, so as to coat and therefore, galvanise the base metal. This entails continuous monitoring and adjustment of additions such as aluminium, silicon, or even mischmetal to the molten bath to counteract the formation of undesirable Fe- $\mathrm{Zn}$ intermetallics. Whilst there are other methods of applying zinc as a means of corrosion protection, these are less prevalent. One such method is electrodeposition, whereby pure zinc is deposited onto the steel substrate using an electric current [47]. However, considering the volume and scale of production for galvanised steel within commercial purposes, costs are critical; consequently, the hot-dipping process is more frequently employed ultimately due to the production costs of such materials. Nevertheless, a galvanising coating acts to not only provide barrier protection, but also sacrificial protection. The barrier aspect of the coating is where the substrate is physically shielded from the environment where it would otherwise corrode. The sacrificial characteristic of the coating acts to corrode in preference to the steel when exposed to the atmosphere; the zinc alloy functions anodically and thus cathodically polarises the steel. This sacrificial protection is fundamental as it also provides corrosion protection at the cut-edge.

\subsubsection{Galvanising alloy chemistries}

There are a multitude of compositions that constitute a galvanising coating, and which coating is best is dependent on factors such as its use, and obviously, cost. Common examples of these coatings are that of hot-dip galvanised steel (HDG), Galfan, and Galvalume. More recent 
chemistries are Galfan-type alloys with additions of magnesium, which will be discussed in detail later in this Chapter.

A typical HDG coating consists of predominantly $\mathrm{Zn}$ and 0.15-0.5 wt.\% Al and is considered to be a standard within the industry. The premise of this coating is that zinc is more reactive than iron according to the electrochemical series; as such, the zinc can preferentially corrode and protect the steel substrate in a sacrificial manner. A coating composed purely of zinc causes the formation of various Fe-Zn intermetallic compounds during the hot-dipping process. These intermetallic compounds which form at the coating/steel substrate interface are brittle and adversely affect the formability of the product. For this reason, small quantities of aluminium introduced into the coating are important, as they inhibit the formation of these intermetallics detrimental to the resultant mechanical properties. Without the additions of aluminium, the coating would be strictly limited in terms of both production and application.

A variation on HDG is the Galfan coating, which once again is primarily made up of zinc, but instead constitutes a larger quantity of aluminium, approximately $4.5 \mathrm{wt} . \%$ Al. There are numerous industrial variations of this alloy, all of which contain a similar zinc-to-aluminium ratio. The increased content of aluminium is advantageous over HDG due to the increased corrosion resistance levels, alongside enhanced formability properties [48]. Under standard solidification conditions, the aluminium is contained within the eutectic and readily forms a protective oxide layer (i.e. $\mathrm{Al}_{2} \mathrm{O}_{3}$ ) under atmospheric conditions [49]. Considering the microstructure of a Galfan alloy, it is typically made up of pro-eutectic zinc-rich dendrites, accompanied by a zinc and aluminium lamellar eutectic, or less common rod eutectic [50], [51]. It is understood that increasing the rate of cooling during production influences the microstructure by reducing the size of both the zinc-rich dendrites and eutectic cells, and the associated inter-lamellar spacing [49]. Other work conducted by Elvins et al. showed that ternary alloying elements introduced into a Galfan coating increased the number and volume fraction of zinc-rich dendrites present in the microstructure [52].

It has been shown that increasing the content of $\mathrm{Al}$ in this type of coating offers dramatic improvement through a reduced extent of anodic attack in an aqueous chloride environment [53]. Considering the beneficial properties of an increased Al content, it is easy to recognise why other galvanising alloys were developed to exploit this. One such alloy coating is known as Galvalume, containing $55 \mathrm{wt} . \% \mathrm{Al}, 43.4 \mathrm{wt} . \% \mathrm{Zn}$, and $1.6 \mathrm{wt} . \% \mathrm{Si}$. The Al content is significant, and this is considered to be the limit in terms of retaining the ability to provide sacrificial protection to the underlying steel substrate. Typically, Galvalume is used in its 
uncoated state as roofing or cladding for buildings, or as a pre-painted coating in the construction industry.

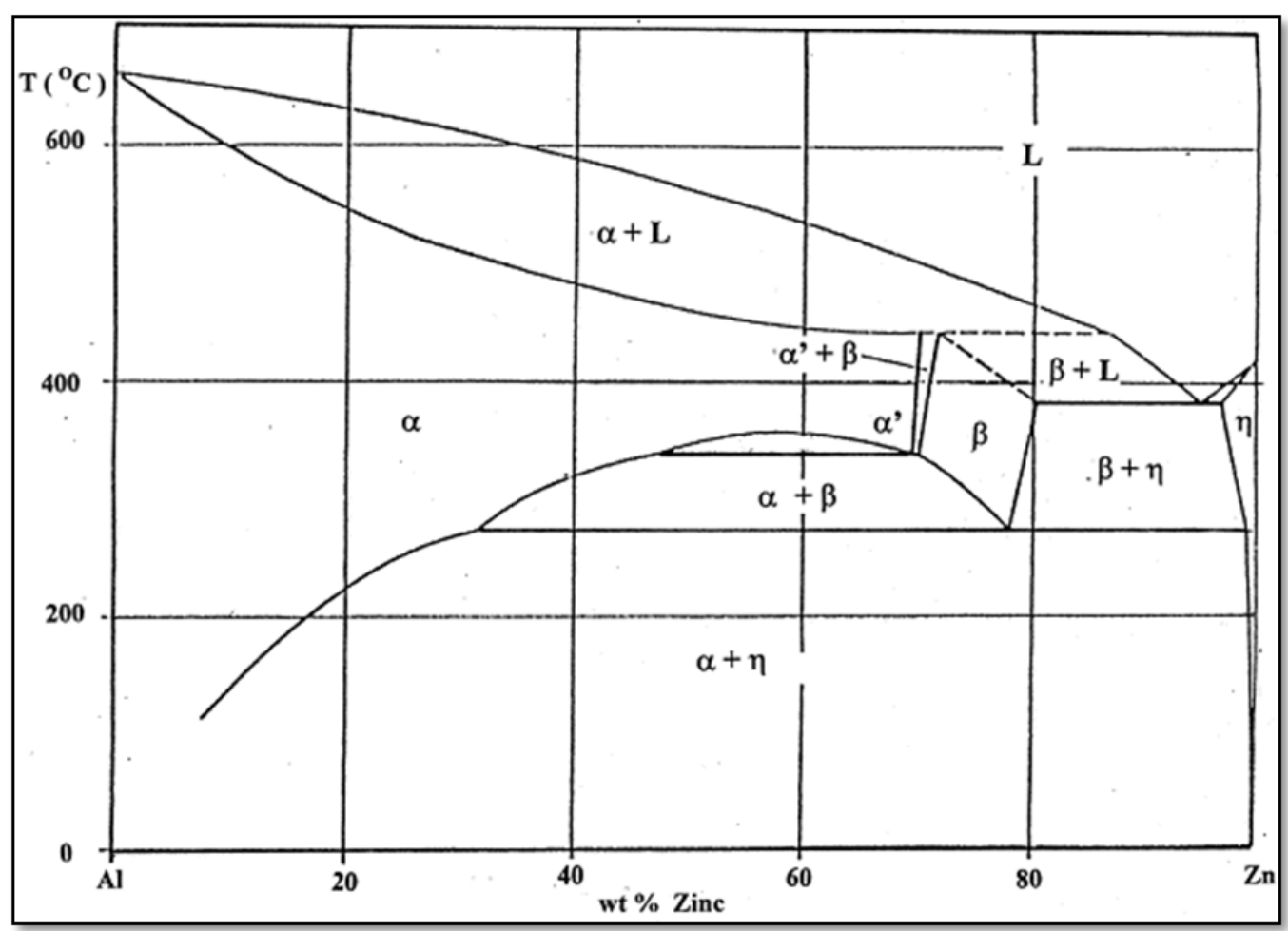

Figure 1.10 Equilibrium phase diagram for $\mathrm{Zn}$-Al system at $25^{\circ} \mathrm{C}$ [54]

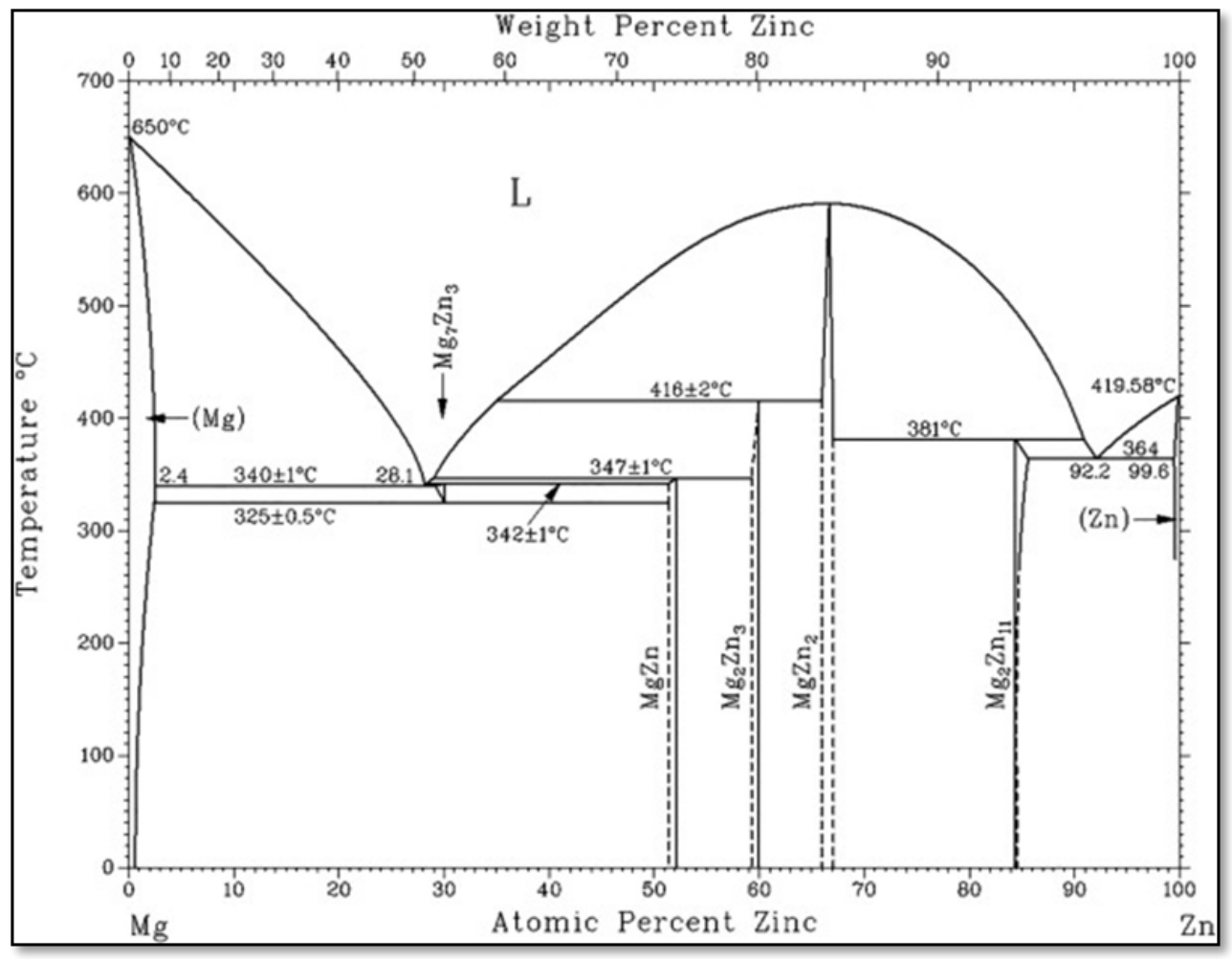

Figure 1.11 Equilibrium phase diagram for $\mathrm{Zn}-\mathrm{Mg}$ system at $25^{\circ} \mathrm{C}$ [55] 


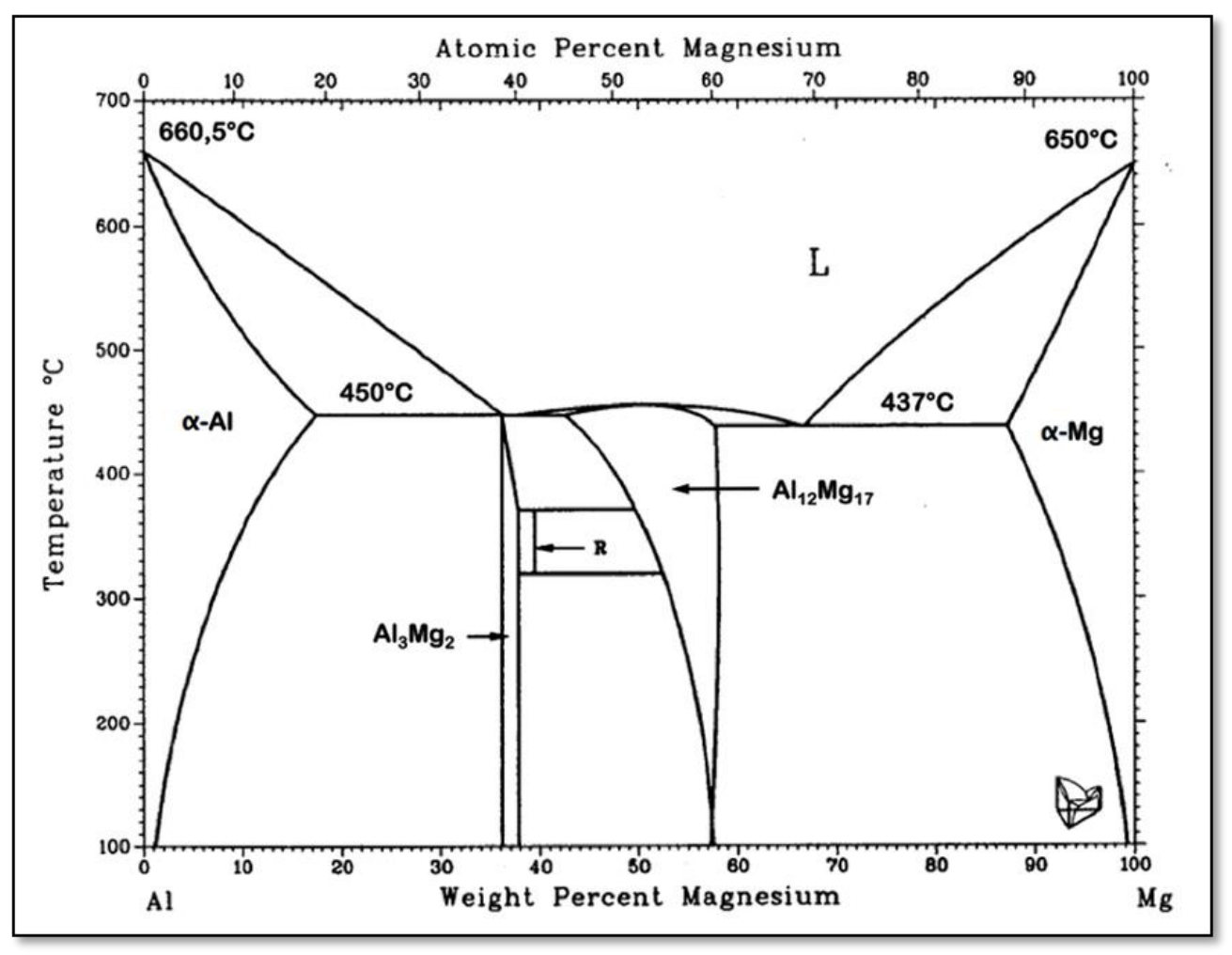

Figure 1.12 Equilibrium phase diagram for Mg-Al system at $25^{\circ} \mathrm{C}[56]$

Equilibrium phase diagrams can be represented in different formats, including that of binary and ternary, and these diagrams can offer predictions of solidification sequences under equilibrium conditions. Considering Figure 1.10, this binary diagram can be utilised to predict the resultant microstructure of a Galfan-type alloy; typically, this might consist of proeutectoid zinc-rich $\eta$ crystals upon interception of the liquidus line, followed by nucleation and growth of eutectic constituting lamellae of $\eta$ zinc and $\beta$ aluminium [57]. In agreement with this, such an alloy would possess a microstructure of primary zinc dendrites amongst a eutectic matrix. Whilst this is useful for a binary alloy, it is somewhat limited when considering a ternary composition, but equally may provide some indication of phase formation in such alloys. For the zinc-magnesium-aluminium alloy compositions studied in this work, a microstructure not dissimilar to the Galfan alloy described above might be expected based upon Figure 1.10; the likely differences would exist as a greater volume fraction of the zinc-rich $\eta$ phase, and a corresponding reduction in $\beta$ phase within the eutectic of the ternary alloy. The phase diagram for Zn-Mg (Figure 1.11) suggests that cooling beyond the liquid phase would yield partial solidification, permitting nucleation and growth of a zinc-rich phase. Continued cooling would next bring about solidification of any remaining liquid to a eutectic, constituting a zinc-rich phase and $\mathrm{Mg}_{2} \mathrm{Zn}_{11}$. The Mg-Al equilibrium diagram (Figure 1.12) would suggest the independent formation of $\alpha$ aluminium and $\alpha$ magnesium phases when considering the overall contribution to the microstructure from all three phase diagrams, with the potential for minor 
amounts of intermetallic phase formation. All of these predictions are based upon equilibrium cooling conditions however, and the hot-dipped galvanising process conventionally makes use of rapid cooling following coating application via the galvanising bath. Therefore, these alloys do not typically follow equilibrium cooling conditions and may not be entirely representative of the microstructural make-up of the alloy.

The complex nature of the ZMA system regarding phase equilibria means that the zincmagnesium-aluminium system may not necessarily be simplified to binary phase diagram interpretation. Accordingly, the ternary phase diagram may be more representative for such a ternary alloy system, as given in Figure 1.13 calculated by Petrov et al [58]. The final microstructure for the alloying quantities considered within this work would be composed of a $\mathrm{Zn}$-rich phase, an Al-Zn phase, and a $\mathrm{Mg}_{2} \mathrm{Zn}_{11}$ phase. Once again, this is based upon equilibrium solidification conditions which did not likely transpire for a hot-dipped product. Consequently, it would be expected that the resultant microstructure may differ for the alloys of interest being studied. As described, it may be expected during equilibrium cooling conditions that $\mathrm{Mg}_{2} \mathrm{Zn}_{11}$ forms as part of this type of alloy. However, the high cooling rate associated with the hot-dip galvanising process may act to inhibit the transformation of the Laves phase of $\mathrm{MgZn}_{2}$, to $\mathrm{Mg}_{2} \mathrm{Zn}_{11}$. This may explain the prevalence of this phase in the binary eutectic for the alloy range considered in other works [59], [60]. 


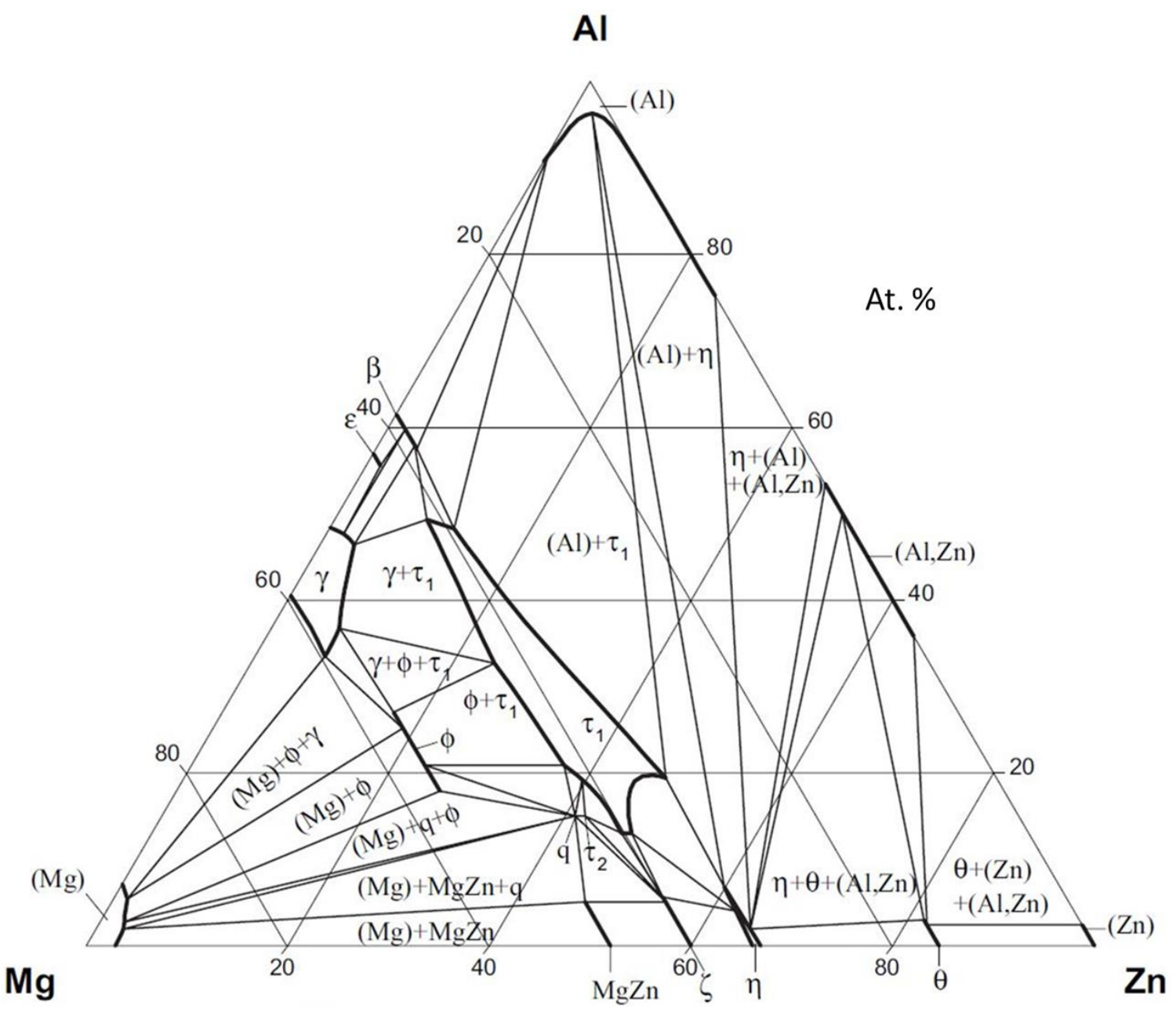

Figure 1.13 Ternary phase diagram for Zn-Mg-Al system [58]

\subsubsection{Zinc-magnesium-aluminium}

More recent developments of coating compositions have resulted in the addition of magnesium into zinc galvanising coatings. Towards the late 1990's, magnesium-containing alloys were made available commercially in the form of Super Dyma and ZAM for use in conjunction with highly corrosive building environments [61], [62], [63, p.]. Research by several of the major steelmakers into zinc-magnesium-aluminium alloys with differing alloy contents is ongoing, primarily considering the hot-dip coating route for both automotive and construction applications [64]. Although, other studies have also held focus on physical vapour deposition (PVD) for Zn-Mg coatings, and this is being extended into Zn-Mg-Al coatings [47], [65], [66]. Previous work has demonstrated that small ternary additions of Mg up to 0.05 wt.\% in a Galfantype alloy were in fact detrimental to the cut-edge corrosion performance, resulting from changes to the microstructure [52]. Conversely, larger alloying additions of magnesium have shown great promise for corrosion resistance levels comparative to conventional galvanising alloy compositions. Results obtained by Prosek et al. even indicated that a coating weight value 
of half or less was required by $\mathrm{Zn}-\mathrm{Mg}$-Al (ZMA) alloys without conceding a fall in performance compared to a traditional HDG coating [61].
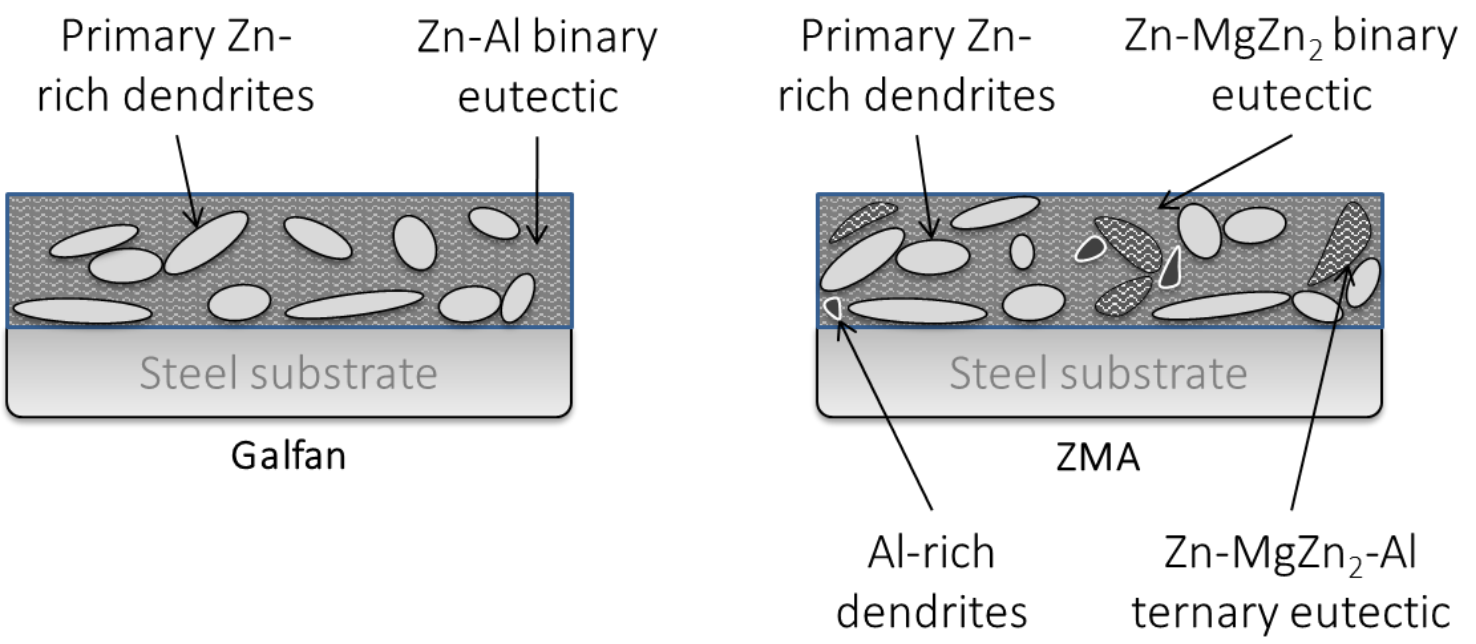

Figure 1.14 Schematic representation of typical microstructure at the cut-edge for Galfan and ZMA coatings

Initial work into magnesium additions exhibited impressive improvements with regard to time to red rust formation under salt spray and cyclic corrosion testing conditions, compared to zinccoated steels [47], [67]-[69]. More recent research by LeBozec et al. revealed that the durability of ZMA coatings is more pronounced depending on the testing conditions and environmental configuration [70]. It was demonstrated that magnesium-containing alloys exhibited obvious performance improvements in environments with higher salt loads; under lower salt loads, those improvements were less apparent relative to conventional coatings. LeBozec et al. also noted that the beneficial behaviour associated with ZMA was more marked for open testing conditions rather than confined ones [70]. Amongst the literature pertaining to the addition of magnesium to zinc or zinc alloy coatings, several theories have been suggested, the majority of which are founded on corrosion product analyses [47], [65], [67], [68], [71], [72]. The formation of stable films has been shown to proceed under salt spray testing conditions, forming an adherent surface layer containing both zinc and aluminium in a ZMA alloy [68]. Prosek et al. studied the composition of corrosion products for both conventional and ZMA alloys under chloride-containing model atmospheric conditions [72]; comparative to HDG, the alloyed coatings presented an increased presence of carbonate corrosion product, with hydrozincite dominating on ZMA in place of simonkolleite. This was accompanied by the formation of layered double hydroxides (LDH). Another body of work performed by Persson et al., again considered the corrosion product analysis of a ZMA coating, but instead focused on the initial stages of atmospheric corrosion [73]. This study corroborated the presence of LDH on 
a ZMA coating, formed by initial dissolution of $\mathrm{Mg}$, Al and $\mathrm{Zn}$. It was concluded that $\mathrm{ZnO}$ formation was ultimately hindered as a consequence, which agrees with the theory outlined by Prosek et al. [72]. There is also agreement in that a buffering effect is instigated by the $\mathrm{Mg}$ and Al content, preventing simonkolleite formation. The electrochemical behaviour of the phases present in magnesium-containing zinc alloys (i.e. $M g Z n_{2}$ ) have been investigated, explaining the increased resistance provided during organic coating delamination conditions [74]. Additional work considering the atmospheric corrosion of galvanising alloys reported formation of LDH at early stages, with hydrozincite and simonkolleite appearing after several hours of exposure [75]. However, immersion conditions of corrosion would be less likely to offer the benefits realised by Mg during atmospheric corrosion as a result of the higher solubility of such compounds [75]. Additionally, the buffering effect of $\mathrm{Mg}$ at cathodic sites is believed to be less effective than in atmospheric conditions, affording more opportunity for zincite formation [75]. The combination of layered double hydroxides, hydrozincite, and simonkolleite were also found during investigations by Salgueiro Azevedo et al [76]; these corrosion products were observed under conditions of salt spray testing and at various chloride loads.

As previously outlined, the addition of small quantities of magnesium to a zinc alloy resulted in alterations to the microstructure, ultimately affecting its corrosion performance [52]. Given the understanding that a variety of microstructural phases can form as a result of magnesium additions, it is necessary to characterise the impact of such phases on corrosion performance. A zinc coating containing 1-2 wt.\% Mg and 1-2 wt.\% Al produced by hot dipping has been examined [77]; the microstructures constitute a Zn phase, a binary Zn/Al eutectic, a $\mathrm{Zn} / \mathrm{Al} / \mathrm{MgZn}_{2}$ ternary eutectic, and $\mathrm{MgZn}_{2}$. In a ZMA alloy of similar composition, Sullivan et al. found that $\mathrm{MgZn}_{2}$ phases were preferentially attacked under aqueous corrosion conditions [78]. As discussed in Chapters 3 and 4, and in [79], some efforts have been made to approach this.

\subsection{Electrochemical scanning techniques}

When considering the corrosion of a metal or alloy within aqueous environments, these particular techniques can prove incredibly useful as they are able to provide insight into the mechanisms of corrosion in addition to the kinetics of these reactions. The primary benefit associated with these techniques is the ability to electrochemically 'map' the surface of a sample; traditional electrochemical techniques indicate bulk corrosion surface events instead. As a general rule, such techniques employ a probe, or micro-tip electrode, scanning at a given distance from the surface of the corroding metal of interest. As the probe rasters across the metallic surface, its physical profile and the values of selected quantity can be simultaneously 
recorded for each individual measurement. This enables a map of the scanned area to be generated from the collation of this data, whereby differences in local surface corrosion activity can be deduced.

\subsubsection{Scanning Vibrating Electrode Technique (SVET)}

This electrochemical scanning technique is considered an iteration on the SRET (Scanning reference electrode technique) and was utilised for the study of the alloys of interest within this area of work. It enables the monitoring of surface corrosion in an aqueous electrolyte and can generate mechanistic understandings of surface corrosion events, which is highly suitable for this field of investigation. For steels encompassing either zinc-based coatings, or organic coating layers, SVET has been used extensively to explore such systems and characterise their cut-edge corrosion performance [49], [52], [80]-[84]. Corrosion behaviour assessment by SVET also offers similar predictions to long-term alternative weathering techniques [85].

When a metal is submerged in an electrolyte, corrosion has the opportunity to develop on the exposed surface, generating localised corrosion features. Depending on the extent of the reaction, these discrete features can be detected. The resultant development of an anode can effectively output ionic current that flows towards remote cathodic areas, and the electrical circuit is completed via the flow of electrons through the substrate metal. The flow of this ionic current can be envisaged as lines of iso-potential that can be intersected by the probe and thus measured by the SVET. The metallic sample of interest is realised to have negligible resistance and consequently, is considered as a plane of constant potential. However, resistance levels will be significantly higher in solution (i.e. the electrolyte); the ionic current flux consequent of anodic activity will give rise to ohmic potential gradients emergent in solution. The iso-potential lines, normal to the ionic current flux can be envisaged as represented in Figure 1.15. Accordingly, the potential and ionic current distribution can be derived via the Laplace equation:

$$
\nabla^{2} E=0
$$

$$
E=\text { potential }
$$

$$
i=-\kappa \nabla E
$$

$\mathrm{i}=$ current 
$\mathrm{K}=$ solution conductivity

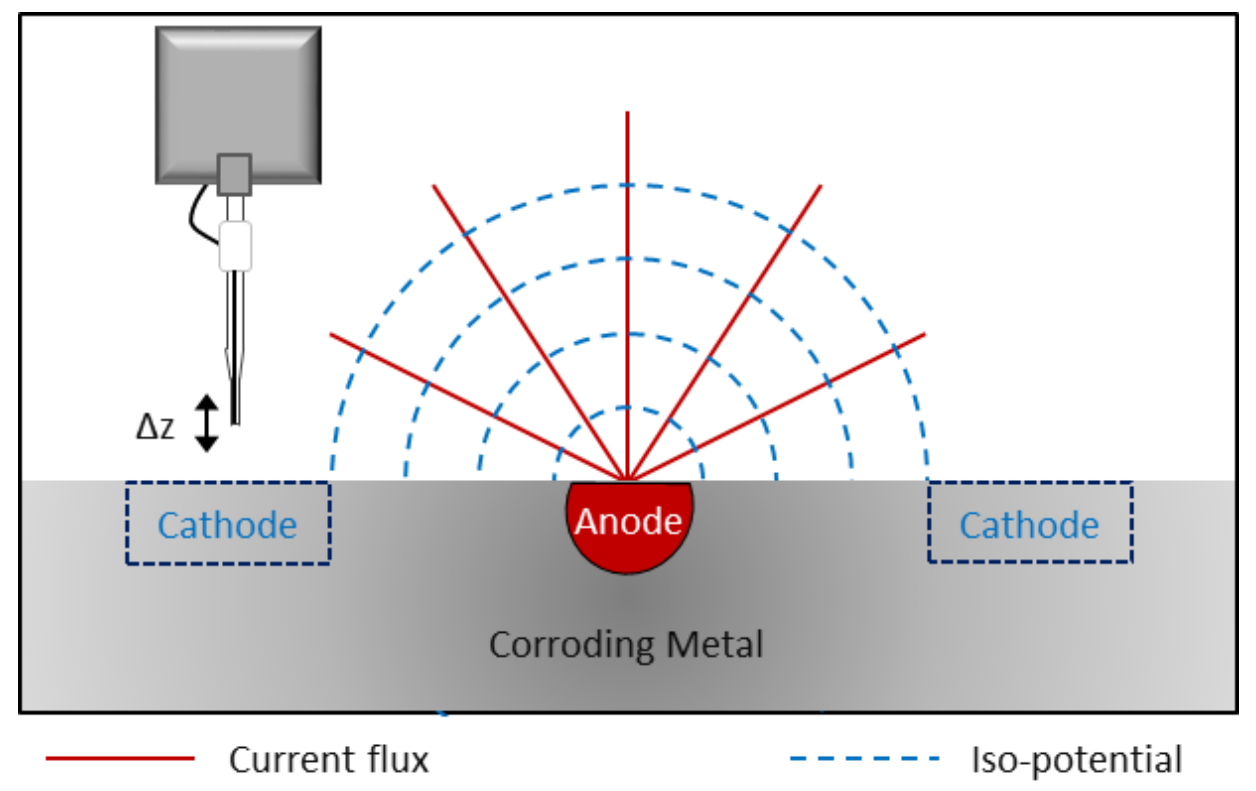

Figure 1.15 Schematic diagram of SVET operation principles above a point anodic site 


\subsection{Thesis aims}

This body of work has been conducted with the following aims in mind, to realise:

1) The effect of $\mathrm{Mg}$ and $\mathrm{Al}$ alloying additions to the microstructure and corrosion rate of commercial ZMA alloys,

2) The role of varying electrolyte $\mathrm{pH}$ condition on the corrosion behaviour of a ZMA alloy,

3) The effects of a sodium phosphate corrosion inhibitor on the ZMA alloy system at varying concentrations,

4) The impact and action of a prospective environmentally-friendly amino acid inhibitor addition within the ZMA system, and

5) The influence of a rare-earth metal inhibitor addition to the corrosion performance of a commercial ZMA system. 


\section{Chapter 2 Experimental procedures}

\subsection{Materials}

\subsubsection{Metallic specimens}

The primary set of materials used for the work completed was a variety of zinc-magnesiumaluminium alloy coatings on steel substrate. These coatings were $10 \mu \mathrm{m}$ in thickness and manufactured under production line conditions, all of which were supplied by the French Corrosion Institute via commercial producers.

\subsubsection{Sample preparation}

\subsubsection{Zinc-magnesium-aluminium samples}

The ZMA coated samples of varying composition were guillotined to approximately $20 \mathrm{~mm} \times 20$ $\mathrm{mm}$ coupons prior to mounting. The mounting process for these coated steel coupons was that of pressure mounting in a non-conductive phenolic resin powder. The thickness of the ZMA coating precluded any mechanical grinding using grit paper, so polishing with a $1 \mu \mathrm{m}$ diamondbased suspension and polishing cloth was performed. This was to provide a flat, consistent surface free of oxide layers and contaminants from the manufacturing process. Samples were cleaned thoroughly via rinsing with distilled water, followed by ethanol. Any samples that underwent any form of imaging were exposed to an etchant in the form of dilute nitric acid in ethanol ( $2 \%$ Nital) as a means of revealing microstructural attributes through preferential attack of grain boundaries. The sample cleaning procedure was repeated as necessary.

\subsubsection{Chemicals and materials}

Table 2.1 List of items used throughout body of work for experimental purposes

\begin{tabular}{|c|c|c|}
\hline Material, Chemical & Supplier & Purity, Grade \\
\hline Cerium chloride heptahydrate, $\mathrm{CeCl}_{3} \cdot 7 \mathrm{H}_{2} \mathrm{O}$ & Sigma-Aldrich & $99.9 \%$ \\
\hline De-ionised water, $\mathrm{H}_{2} \mathrm{O}$ & In situ & $18 \mathrm{M} \Omega$-cm \\
\hline Glycine, $\mathrm{NH}_{2} \mathrm{CH}_{2} \mathrm{COOH}$ & Sigma-Aldrich & $\geq 99 \%$ \\
\hline Hydrochloric acid, $\mathrm{HCl}$ & Sigma-Aldrich & ACS Reagent \\
\hline L-tryptophan, $\mathrm{C}_{11} \mathrm{H}_{12} \mathrm{~N}_{2} \mathrm{O}_{2}$ & Sigma-Aldrich & $\geq 98 \%$ \\
\hline Sodium chloride, $\mathrm{NaCl} \mathrm{Na}_{2} \mathrm{Cr}_{2} \mathrm{O}_{7} \cdot 2 \mathrm{H}_{2} \mathrm{O}$ & Sigma-Aldrich & $\geq 99 \%$ \\
\hline Sodium dichromate dihydrate, $\mathrm{NaOH}$ & Sigma-Aldrich & $\geq 99.5 \%$ \\
\hline Sodium hydroxide, $\mathrm{NaOH}_{3}$ & Sigma-Aldrich & $\geq 98 \%$ \\
\hline Sodium phosphate, $\mathrm{Na}_{3} \mathrm{PO}_{4}$ & Sigma-Aldrich & $\geq 99 \%$ \\
\hline Extruded PTFE tape & $3 \mathrm{M}$ & - \\
\hline
\end{tabular}


2.2 Study of corrosion behaviour using the Scanning Vibrating Electrode Technique (SVET)

When considering cut-edge corrosion on steels that incorporate either organic layers or simply a zinc-based coating, previous research has demonstrated the usefulness of SVET when exploring such systems [49], [52], [80]-[84]; this allows the ability to generate mechanistic understandings of these surface corrosion events. Furthermore, the SVET has demonstrated comparable results for the prediction of corrosion behaviour over extended periods of time in comparison with alternative weathering techniques [85]. Essentially, the SVET is able to resolve localised corrosion events taking place on the surface of a metallic sample under immersion conditions. Surplus to this, SVET can provide values of anodic current density for each experiment, which in turn can produce a corresponding value for metal loss in a semiquantitative fashion. Accordingly, this metal loss value may be utilised for comparing relative performance of different corrosion inhibitor additions in electrolyte or variation in alloy composition. With regard to the area of the sample being studied by SVET, the surface was masked using an extruded PTFE tape, with a ca. $10 \mathrm{~mm} \times 10 \mathrm{~mm}$ area exposed and available for scanning. Additionally, the error bars included in any figures representing SVET-derived metal loss values are the most extreme values realised during repeated experiments of the studied parameters.

\subsubsection{SVET apparatus and point current source response}

As given in the schematic diagram of Figure 2.1, the SVET employs a platinum wire microtip, $125 \mu \mathrm{m}$ in diameter, enveloped in borosilicate glass. This probe microtip vibrates at a constant amplitude, frequency, and height above the surface of a sample whilst immersed in an electrolyte bath. During operation, the SVET detects alternating potential at the frequency of vibration, which is proportional to the vertical component of the potential gradient in solution. The lock-in amplifier (EG\&G Instruments 7265) governs the frequency of vibration whilst also measuring the signal received at the probe tip. The lock-in amplifier also provides the vibration through an external amplifier, the frequency of which is $140 \mathrm{~Hz}$, with a probe vibration amplitude of $c a .25 \mu \mathrm{m}$ by the use of an appropriate drive voltage. On the corroding sample surface, anode and cathode locations can be resolved at known points, in addition to their intensities. The sample surface is scanned with the probe tip at a set height of $100 \mu \mathrm{m}$ in stepped intervals using a micro manipulator stage, in conjunction with a PC. Ionic current flux originating at any anode existing on the corroding surface passes through the electrolyte and sets up potential fields in solution. The SVET can then record an alternating potential by crossing these lines of current flux. The signal measured by the SVET probe is directly 
proportional to the vertical component of ionic current density in the direction of the probe vibration (z axis). In effect, point current densities can be calculated from the potential data via the use of a calibration factor, which in turn can be realised as a means of determining corrosion levels occurring on the sample surface.

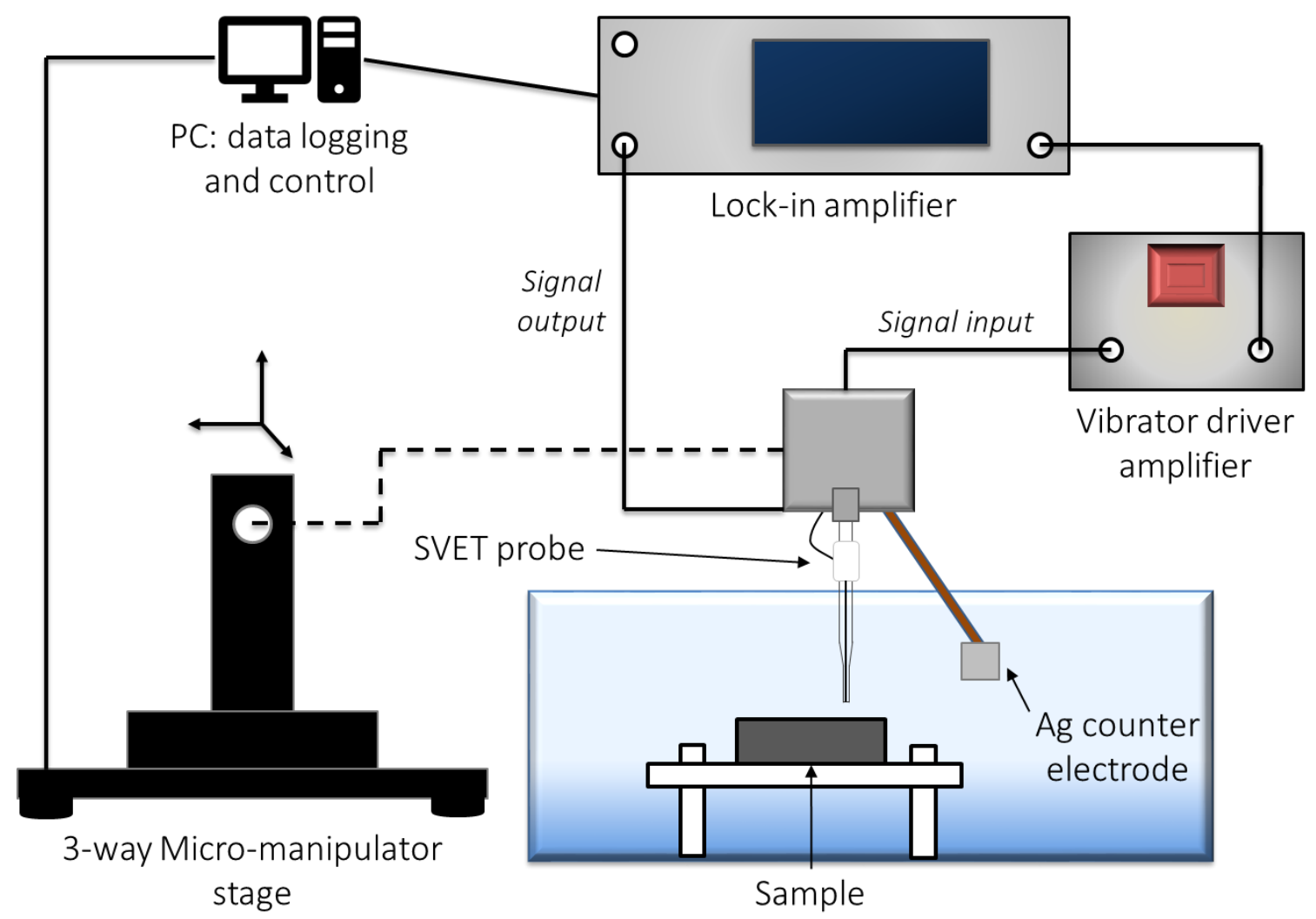

Figure 2.1 Schematic representation of SVET apparatus

With regard to the operation of the SVET, it is understood that potential fields in solution are generated by ionic current flux, which originate from an anodic current source. The SVET microtip registers alternating potentials at the frequency of vibration, of which are recognised to be proportional to the electrical field strength, $\mathrm{F}$, or the potential gradient normal to a surface anodic site. Equation 2.1 denotes the change in electrical field strength, F, with proximity from a point current source:

$$
\mathrm{F}=\frac{\mathrm{dE}}{\mathrm{dz}}=\frac{\mathrm{iz}}{2 \pi \mathrm{k}\left(\mathrm{x}^{2}+\mathrm{y}^{2}+\mathrm{z}^{2}\right)^{1.5}}
$$

$\mathrm{k}=$ electrolyte conductivity

$x, y, z=$ distance in each coordinate plane

$\mathrm{i}=$ current source 


$$
F_{\max }=\frac{\mathrm{i}}{2 \pi \mathrm{kz}^{2}}
$$

Equation 2.2 denotes that the maximum electrical field strength measurable will exist at the origin of a point current source. The relationship between probe height and $F_{\max }$ also means that the distance of the SVET probe in relation to the sample surface is vital.

It is necessary to realise that SVET does have limitations in terms of resolution. In effect, the spatial resolution of the SVET is the ability of the probe to distinguish the distance separating two localised corrosion features. Due to the Gaussian distribution of the normal electrical field strength from the point source origin, the theoretical spatial resolution of the SVET can be determined by the full-width half-maximum $(w h m)$ of the signal peak. Considering a scan height of $100 \mu \mathrm{m}$ used throughout the entirety of this work, a theoretical resolution of $153 \mu \mathrm{m}$ would result, as considered in other work [86]. However, the observed whm value in practice is typically realised to be $0.27 \mathrm{~mm}$, providing a resolution of approximately $250 \mu \mathrm{m}$. This is in part attributable to the SVET microtip dimensions, of which the platinum microdisc has a diameter of $125 \mu \mathrm{m}$. Recognition of this limit in spatial resolution explains that the SVET will be unable to view localised corrosion features separated by a distance less than that stipulated. This highlights the ability of SVET to view localised corrosion activity rather than generalised activity, as during the latter, potential gradients in solution terminate prior to SVET probe intersection.

\subsubsection{SVET calibration}

The SVET registers and records potential gradients in solution in $\mathrm{nV}$, which alone is not incredibly useful. In order to generate meaningful data, these voltages must be converted to current density values in $\mathrm{A} \mathrm{m}^{-2}$ through a means of calibration. A method of calibration can be achieved by the use of a point current source. Under conditions that correspond to Ohm's law, the current flux in the direction of vibration $\left(\mathrm{j}_{2}\right)$ is correlated with $\mathrm{V}_{\mathrm{pp}}$, the peak-to-peak voltage signal measured by SVET. At a point current source, current emerges from its centre evenly and with a hemispherical profile. Therefore, the current density in the plane of the $\operatorname{scan}\left(\mathrm{j}_{2}\right)$ can be calculated by dividing the applied current by the hemispherical surface area, as set out in Equation 2.3:

$$
j_{z}=\frac{\mathrm{i}}{2 \pi \mathrm{z}^{2}}
$$


$\mathrm{i}=$ current source

$z=$ height of probe above current source

Plotting $\mathrm{V}_{\mathrm{pp}}$ registered by the SVET against the above equation (2.3) provides a method of calibration by converting the SVET voltage signal to a current density value in the direction of probe vibration $\left(\mathrm{j}_{2}\right)$.

The outlined means of calibration is calculated prior to each SVET experiment and is completed with the use of a two-compartment cell, as set out in Figure 2.2. Each compartment individually contains a $1 \mathrm{~cm}^{2}$ platinum electrode, as well as the electrolyte being used within the experiment. Both compartments are linked by a glass tube, which is vertically positioned and has an internal diameter of $5 \mathrm{~mm}$. The SVET tip is lowered into the vertical opening of the tube and a range of currents are applied using an in-house manufactured nano-galvanostat. The known area of the glass tube allows current densities to be calculated for each applied current. It is assumed that the orientation and profile of the tube creates uniform current density as the lines of current flux align parallel to its length, and thus the direction of SVET probe vibration. The voltage measured by the SVET for each applied current density is recognised to vary in a linear fashion, as shown by Figure 2.3. Therefore, the gradient obtained from the plot of measured SVET voltage against current density is considered as the calibration factor. This calibration factor can be applied to the SVET signal recorded during the length of the experiment in its given electrolyte to produce a value for current density in the plane of scan. Whilst there are other means of acquiring a calibration factor, this is considered an efficient means of converting the voltage data detected by SVET into current density values. 


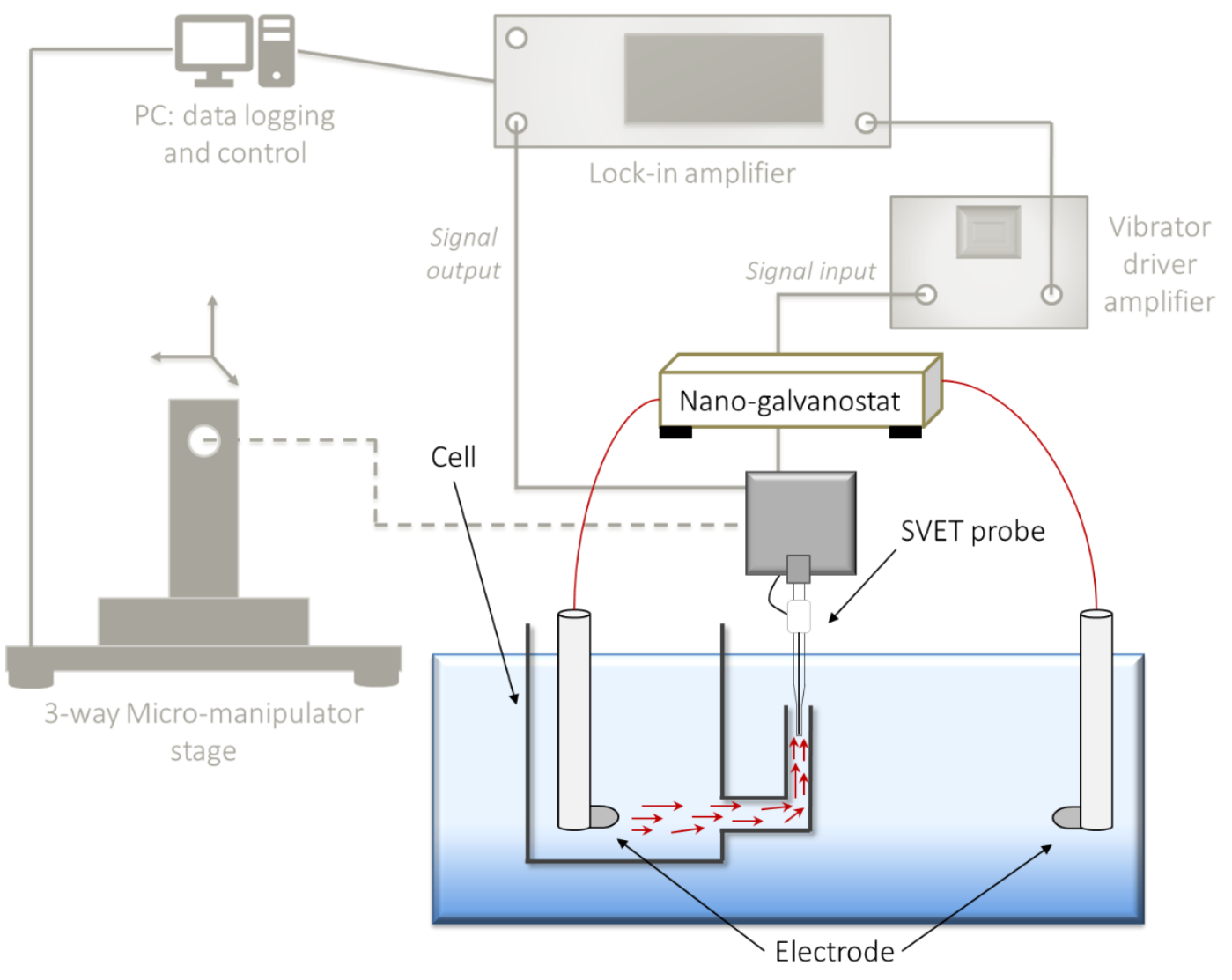

Figure 2.2 Schematic depiction of an SVET calibration setup using a two-compartment cell

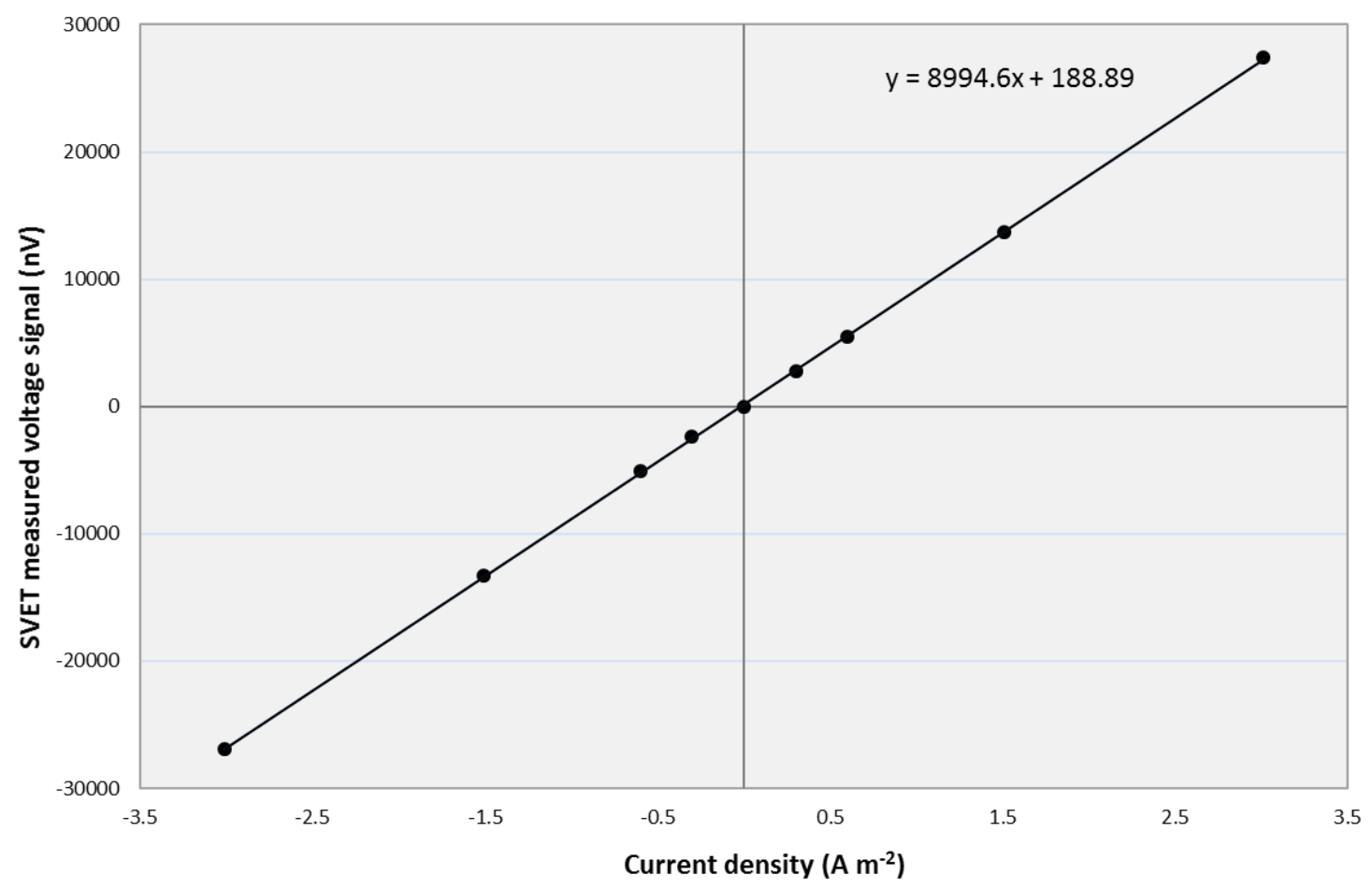

Figure 2.3 Example SVET calibration plot in $1 \% \mathrm{w} / \mathrm{v} \mathrm{NaCl}$ electrolyte 


\subsubsection{SVET operation for the examination of corrosion behaviour}

The SVET was utilised to produce both qualitative and semi-quantitative data of the corrosion behaviour of the range of metallic samples used throughout this body of work. These tests were conducted under immersion conditions, whereby the samples are submerged in their relevant electrolytes and the surface scanned by the platinum SVET microtip. The specific electrolytes used during such experiments are stated within the individual chapter, including any corrosion inhibitor additions. Scans were performed hourly over a total 24-hour period, creating a set of time-dependant corrosion surface maps per experiment. The dissolved oxygen concentration in the bulk solution was presumed to be the equilibrium concentration for air saturated water of $2.8 \times 10^{-4} \mathrm{~mol} \mathrm{dm}^{-3}[79]$. All experiments were conducted in laboratory, at a controlled temperature of $25^{\circ} \mathrm{C}$.

The SVET platinum microtip is bridged by a Teflon sheath to a glass push rod, which is fixed to the cone of a loudspeaker and functions to deliver the vibration to the microtip. The glass capillary push rod and loudspeaker are enclosed in a header box made up of mu-metal, and acts to shield against electromagnetic leakage generated from operation of the loudspeaker. In effect, this is to minimise any signal interference due to the sensitive nature of the data readings obtained from SVET.

Under conditions of localised corrosion, emergence of currents from point anodes on a metallic surface bare huge resemblance to point current sources. This clarifies the SVETs ability to resolve and monitor corrosion activity occurring on a sample surface. The SVET was utilised throughout each chapter of work as a means of evaluating corrosion performance of each metallic surface in an aqueous medium.

\subsubsection{SVET surface profiling}

Equation 2.3 demonstrates that the SVET signal is proportional to the inverse square of the scan height, $z$, and thus careful control of this height is critical for each experiment to ensure accurate data readings. Accordingly, the sample of interest must either be height profiled, or levelled and therefore seated in a plane normal to the direction of probe vibration. The latter option comprises a manual levelling procedure, and necessitates a flat, bevel-free surface. To perform manual levelling, the sample was fastened to a platform using small nylon screws, and the square platform itself levelled via adjustment of four larger nylon screws installed towards its corners. At each corner of the exposed sample surface, the SVET probe was moved in the $z$ direction to periodically establish the distance of the sample from the resting position of the probe. Repeating this procedure in each corner of the sample allows the probe to be at a 
consistent height of $100 \mu \mathrm{m}$ above the surface, and for the means of this type of experiment is considered level.

Alternatively, the SVET has the capability of height profiling via an interpolated or 3D height scan, the latter of which is used for a non-planar sample. However, for the purposes of this body of work, an interpolated height scan procedure was employed due to the use of flat, polished samples. This form of height scan utilises the loudspeaker incorporated within the SVET head and is measured against a reference loudspeaker. This enables fine fluctuations in the measured signal to be realised. Therefore, a height detection threshold value can be defined whereby interaction of the SVET tip with the sample surface is registered, allowing profiling of the surface. This is accomplished through lowering of the tip in the $z$ direction at a corner until surface detection, and recording the change in height from its resting position. The SVET tip returns to its rest height, and continues this procedure in each corner of the sample, logging the differences in height at each position. The software interpolates these four discrete points in a linear fashion, generating a planar profile for the sample according to the $x, y$ and $z$ positions. When the experiment is commenced to record active corrosion measurements, the height scan profile allows the SVET probe to trace $100 \mu \mathrm{m}$ above the sample surface at each given position.

\subsubsection{SVET data rendering and manipulation}

All data captured from SVET testing was subject to calibration as outlined in 2.2.2. This is to convert the raw SVET signal measured in $\mathrm{nV}$, into more meaningful data in the form of $\mathrm{A} \mathrm{m}^{-2}$. A contouring and surface modelling software package, Golden Software's Surfer 10 was the platform used for mapping of the data. The calibrated data is rendered as a contour map per hourly scan. A false colour is imposed onto the maps to distinguish the locations and intensities of anodic and cathodic surface activity. The magnitude of this activity is then portrayed by the colour intensity, and anodic and cathodic activities are identified by a change in colour from red to blue, respectively. A scale bar is used as a reference point for surface activity, displaying the range of current density values observable for each experiment. This in essence is a spatially resolved map of normal current density occurring on the scanned surface.

Additional meaningful data can be derived from SVET surplus to the normal current density values distributed on the surface $\left(\mathrm{j}_{2}\right)$. For each map of current density distribution $\left(\mathrm{j}_{2}\right)$ throughout an experiment, a corresponding value of anodic-going current $\left(\mathrm{Ia}_{\mathrm{t}}\right)$ can be produced through numerical area integration: 


$$
I a_{t}=\int_{0}^{X} \int_{0}^{Y}\left[j_{z(x, y)}\right] d x d y
$$

$\mathrm{la}_{\mathrm{t}}=$ anodic current

$\mathrm{j}_{\mathrm{z}}=$ SVET-derived current density

$x, y=$ length and width of SVET scan

The resulting value for each individual scan can be considered as the total anodic current emerging from the scanned surface. The sum of these values over the entirety of the experiment can give an indication of the progression of surface corrosion events with respect to time. Per each scan, the value of $l a_{t}$ can in turn be divided by the scanned sample area to give the area-averaged anodic current density, Jat. A value for total estimated metal loss can be produced from these positive anodic current values by the application of Faraday's Law. Whilst the ability to quantify the metal lost from a corroding surface is very beneficial, this is considered a semi-quantitative metal loss value. This consideration is due to the assumption that corrosion activity remains constant between each scan, and the fact that the SVET measures a normal component of ionic current flux rather than direct surface current density. To ascertain a value for charge, according to equation 2.5 , the anodic current determined for an individual scan is multiplied by the scan time:

$$
Q=I x t
$$

$$
\begin{aligned}
& Q=\text { charge } \\
& I=\text { current } \\
& t=\text { time }
\end{aligned}
$$

The calculated charge $(\mathrm{Q})$ can then be entered into Faraday's Law to approximate the total metal loss from a sample. For example, in the case of zinc, total zinc loss (tzl) could be determined as follows:

$$
t z l=\frac{Q}{2 F} x M_{(Z n)}
$$

$Q=$ charge 
$\mathrm{F}=$ Faraday's constant $\left(96487 \mathrm{c} \mathrm{mol}^{-1}\right)$

$\mathrm{M}_{(\mathrm{Zn})}=$ molar mass of zinc $\left(65.28 \mathrm{~g} \mathrm{~mol}^{-1}\right)$

The SVET measured metal loss value is a valuable indicator of trend, but it must be realised that this is not a direct measure of metal loss. That being said, it stands to reason that manipulating the data in this fashion is a powerful tool for comparison of similar alloy systems.

\subsection{Time-lapse optical microscopy}

Time-lapse optical microscopy is a valuable technique that permits in situ observation of surface corrosion events at a microstructural level over a designated time period. A cylindrical PET waterproof shroud is fitted to the lens of a Meiji Techno MT7100 metallurgical microscope and encompasses a glass viewing window on its base. Ultimately, this enables the sample of interest to be monitored under immersion conditions. This experimental setup, depicted in Figure 2.4 , is an iteration based on a previously developed technique [78].

All samples used in conjunction with this technique were prepared as outlined in 2.1.2.1. Subsequently, samples are masked with adhesive PTFE tape, revealing an exposed circular feature of $c a .1 \mathrm{~mm}$ diameter, or $0.785 \mathrm{~mm}^{2}$ area. The sample being studied was affixed to a platform using PTFE tape within a glass receptacle, of which was subsequently filled with $250 \mathrm{~mL}$ of electrolyte so as to fully immerse the sample. The microscope lens was then moved into position above the exposed area of the immersed sample surface to allow images of the microstructure to be captured using the connected Infinity 2 digital camera. An image was captured at two-minute intervals as designated using the time-lapse function of the software, corresponding to the attached camera. Time-lapse experiment duration extended to 17 hours in length or to the point where the microstructure was completely obscured and was no longer viewable, whichever of these transpired first. The microstructural images captured over the experiment duration were then assembled into a time-lapse video using Windows Live Movie Maker software. The time-lapse video is a rapid, and straightforward means of observing the change in surface corrosion events over time. However, for those experiments that employed corrosion inhibitor additions, the experiment duration was extended to 36 hours, or until the distinct features of the microstructure were no longer visible due to extensive corrosive attack.

It is understood that the resolution of an optical microscope is dependent on the numerical aperture of the objective lens, of which is partially dictated by the refractive index of the medium existing between the objective lens and the sample of interest. Accordingly, when utilising optical light microscopy for means of high resolution image capture, it is common 
practice to apply a form of immersion oil. This specialised oil tends to have a high refractive index, and improves the light-gathering ability of the objective lens, and therefore, its resolution. The objective lens used for this form of characterisation is not strictly designed for immersion using water as a medium. Nevertheless, sample images captured under electrolyte solution immersion conditions did not show excessive deviations from those images captured in air. As such, the apparatus was considered more than adequate for the purposes of in situ corrosion monitoring. This was aided by the design, whereby the lens was proximal to the sample surface and the bridging electrolyte layer was not excessive, in order to minimise augmentation of the captured images.

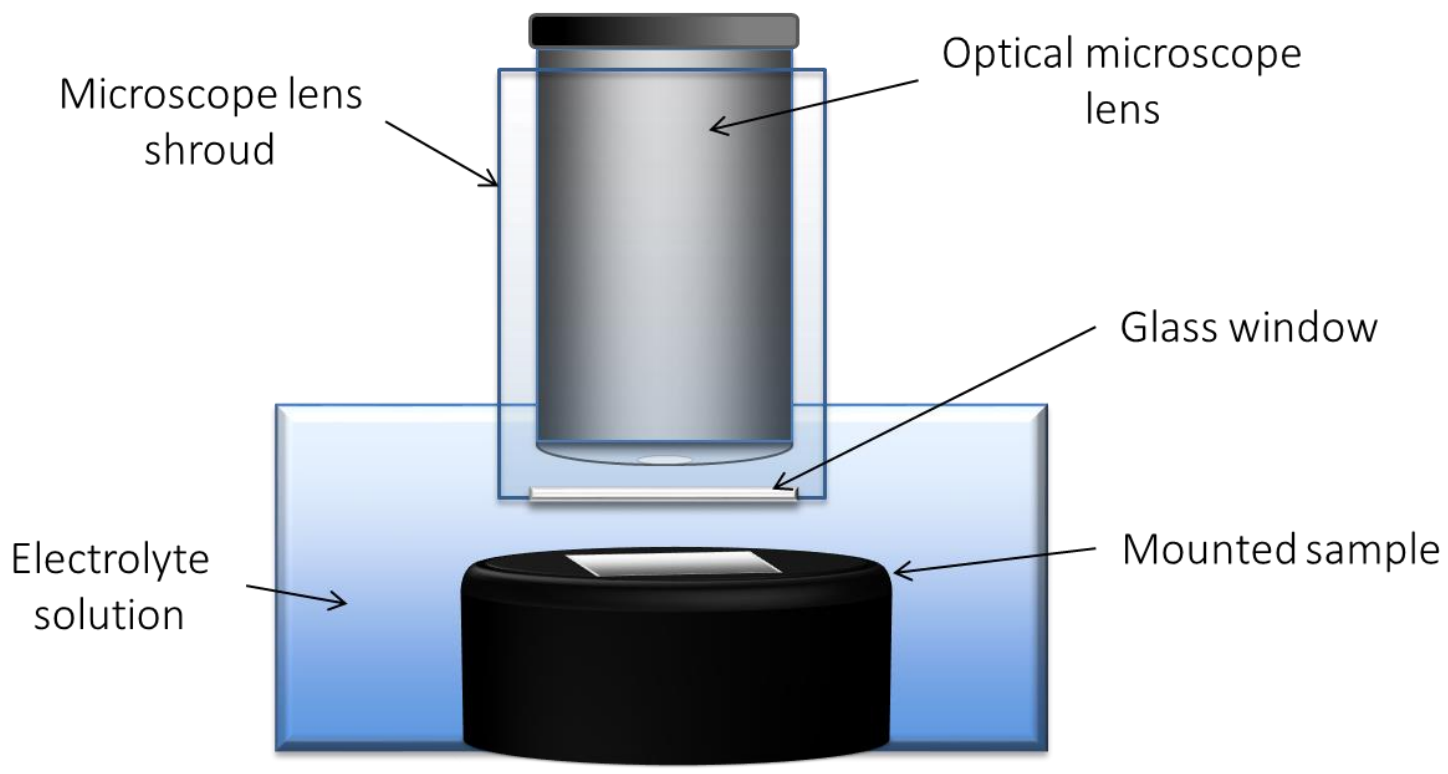

Figure 2.4 Schematic representation of time-lapse optical microscopy setup

\subsection{Scanning electron microscopy and energy-dispersive X-ray spectroscopy}

Scanning electron microscopy (SEM) is a broadly used characterisation technique that is employed for the rendering of high resolution images. This is via emission of a focused beam of electrons that raster a sample surface in a given pattern; the interaction with the sample generates a signal that is realised by the signal detector, which combined with the known beam position can form an image. The SEM utilised during this body of work was a Hitachi TM3000 Tabletop Microscope. The observation condition was set to $15 \mathrm{kV}$ accelerating voltage for imaging of samples, under the Analysis selection. Another widely used technique in the form of energy-dispersive X-ray spectroscopy (EDS) was utilised for means of elemental composition analysis of samples. This was performed using the Bruker Quantax70 EDS apparatus integrated into the SEM suite. This function was primarily used for elemental mapping and validation of the elements present in corrosion products. 


\subsection{Open-circuit potential (OCP)}

The testing of selected metallic specimens using open-circuit potential was conducted as a means of establishing their free corrosion potential in a corrosive electrolyte. This is considered as a two electrode cell, whereby the exposed metal of interest functions as the working electrode (WE); the potential of which is measured by the potentiostat against a standard reference electrode (SRE). The reference electrode used throughout this form of electrochemical testing took the form of a saturated calomel electrode (SCE). The Solartron SI 1280 potentiostat simply acts as a voltmeter, taking measurements of potential over a set timescale.

Each sample was prepared as set out in section 2.1.2.1, whereby these were fitted into an in-house manufactured sample holder, as seen in Figure 2.5. This isolated the metal surface from electrolyte under immersion conditions, with the exception of a circular gasket exposing an area of $0.95 \mathrm{~cm}^{2}$ to any present electrolyte. A contact seated at the rear of the sample holder was used to establish an electrical connection with the WE. The electrolyte used for this series of samples was a $1 \% \mathrm{w} / \mathrm{v}(0.172 \mathrm{M}) \mathrm{NaCl}$ solution neutralised to $\mathrm{pH} 7$ unless otherwise noted. This served as the foundation electrolyte for any corrosion inhibitor additions introduced, of which is described in full in chapters to follow. Unless otherwise stated, each open-circuit potential test was carried out over a 30-minute time-frame to establish stable readings for each sample.

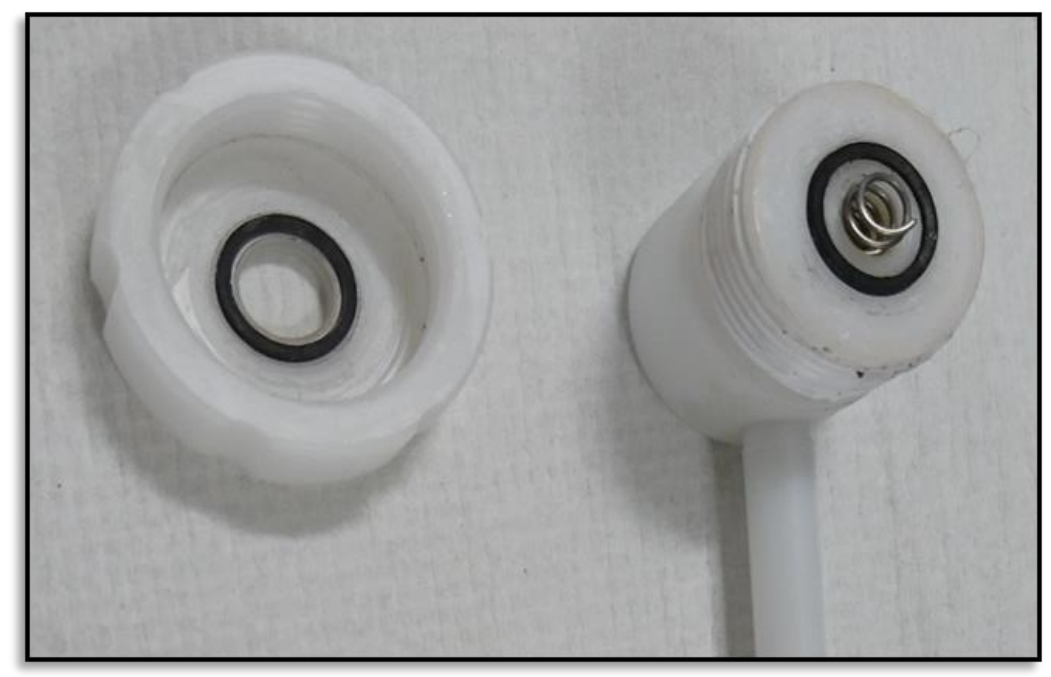

Figure 2.5 In-house manufactured sample holder

\subsection{Polarisation}

Potentiodynamic experiments were performed to observe changes in corrosion behaviour due to polarisation effects. All samples used during this electrochemical characterisation were 
prepared as described in 2.1.2.1, and housed within the custom-made sample holder as detailed in 2.4. The testing exercised a three-electrode cell, all of which were immersed in an electrolyte setup, used in conjunction with a potentiostat (Solartron SI 1280). The three electrodes were comprised of the sample of interest as the working electrode, a saturated calomel electrode serving as the reference electrode, and a platinum gauze sheet functioning as the counter electrode. Under immersions conditions, an external potential is applied to the working electrode at a pre-determined rate and starting point, which was measured against the saturated calomel electrode until the desired potential is reached. The resulting current output is recorded between the working electrode and platinum counter electrode. This form of testing produces a plot of measured current against potential (vs. SCE). The corrosion inhibitor additions introduced to the $1 \% \mathrm{w} / \mathrm{v} \mathrm{NaCl}$ electrolyte throughout the polarisation experiments are detailed in the relevant chapters. The sweep rate for each experiment was $1 \mathrm{mV} \mathrm{s}^{-1}$, and the potential sweep range is specified per chapter.

An additional means of assessing the rate of corrosion via the generation of metal loss values was utilised from data generated from potentiodynamic polarisation experiments. This was performed using Tafel extrapolation from the linear regions of the cathodic and anodic curves. The corrosion current, $I_{\text {corr }}$ was determined at the intersection point between these curves to enable the corrosion rate of the sample (working electrode) to be calculated. This was achieved using Equation 2.6, which was also used for SVET-derived metal loss calculations. To maintain comparability between data sets, zinc was selected as the corroding species for these calculations, and the time period was considered as 24 hours.

\subsection{Gravimetric analysis}

A set of gravimetric analyses were conducted according to procedures as set out in ISO 8407, to assess and substantiate the estimated metal loss data gathered via SVET. Samples were cut into $24 \mathrm{~mm} \times 24 \mathrm{~mm}$ coupons and were polished and etched to the same standard as others used within this investigation. The prepared samples were washed thoroughly with deionised water and then ethanol to remove any residual contaminants obtained from storage, handling and the metallography process. These were dried using a hot air blower and then moved to a desiccator for 24 hours before recording a pre-corroded mass value. Once a mass was recorded for each individual sample, each surface was encased in PTFE tape, excluding a circular feature $16 \mathrm{~mm}$ in diameter, resulting in an exposed area of $201 \mathrm{~mm}^{2}$. Following this, the samples were allowed to corrode via immersion in electrolyte for an allocated time of 24 hours. All electrolytes used during the gravimetric analysis were a $1 \% \mathrm{w} / \mathrm{v}$ sodium chloride $(\mathrm{NaCl})$ solution, with a variation 
in $\mathrm{pH}$ of 3,7 , or 10 . Once the immersion times were elapsed, samples were removed from their electrolyte solution and gently brushed to remove lightly adherent corrosion products whilst being thoroughly rinsed with tap water. This was followed by washing with deionised water, and finally ethanol. The corroded samples were then submerged in deionised water in an ultrasonic bath for five minutes to remove water-soluble corrosion products. Subsequently, samples underwent a chemical cleaning procedure to remove remaining corrosion products, whereby they were individually immersed in a saturated glycine solution whilst irradiated in an ultrasonic bath, in 4-minute intervals. Following each interval, samples were cleaned with tap water, deionised water, and ethanol, where they were dried with a hot air blower and then placed into a desiccator for a further 24 hours. Upon completion of each corrosion product removal and cleaning cycle, the sample mass was recorded. These cleaning cycles were repeated until all corrosion products were removed from the sample following visual inspection. This produced a mass loss for a given area that can be contrasted with that measured by SVET to evaluate the reliability of such results. Any error accounted for in the results from this type of experimentation were based on the fluctuations observed during measurements between a control sample and the samples under scrutiny.

\subsection{Image analyses}

The digital images captured through means of optical microscopy were analysed to extract meaningful quantitative data. This was performed for purposes of both time-lapse optical microscopy, and optical light microscope images corresponding to different microstructures per alloy content variation. Regarding the latter, volume fraction analysis of phases present was performed using Photoshop CS6 software. A user-adjustable contour selection feature was used to designate threshold boundaries whereby only phases of interest were highlighted. The areas associated with the phases of interest were subsequently measured as a percentage of the total area present for each image. Procedurally, the time-lapse microscopy analysis was similar in the use of the contour selection tool via the Photoshop CS6 software. This analysis differed in that the darkened area associated with the anodic attack was highlighted instead of specific microstructural phases. The area of these highlighted regions was measured with respect to a calibrated reference image of known dimensions. Due to the large quantity of images produced from the time-lapse microscope experiments, only a selection of images was analysed during this process. Further information regarding the time intervals and corresponding images are designated within the relevant chapters. The error bars included in image analysis results are based on the tolerances dictated by the selection tool of the Photoshop software, which utilises a threshold value. 


\subsubsection{SVET image analysis}

The SVET current density plots were analysed to provide an indication of approximate size of anodic features, and to monitor any change in size for such features over time. Using

Photoshop CS6, the length of a prominent point anode was measured about its midpoint, taking the largest value. Typically forming as a point anode, where possible, a ring-profile corrosion feature in the form of an anodic ring with a cathodic inner feature was measured to represent the activity on the surface. These measurements were plotted at 4-hour intervals for the 24hour experiment length, except for analysis of the hour 1 scan in place of hour 0 , due to the minimal activity normally visible on the corroding sample surface. In the case of progressively coalescing anodes, a boundary for a single anode was estimated until there was no appreciable boundary remaining to measure individually. 


\section{Chapter 3 Influence of composition variation on microstructure and corrosion behaviour of zinc-magnesium-aluminium (ZMA) galvanising alloys}

\subsection{Introduction}

The utilisation of pre-finished steel products is extensive and confirms the requirement for their continual development. Galvanising alloys are recognised to be a major component of these pre-finished, organically-coated steels, which are utilised across the globe and in a range of industries. The widespread use of these alloys stem from the ability of the coating to sacrificially protect the underlying steel substrate through preferential corrosion of the zinc alloy under environmental exposure conditions. When these coated systems are implemented within the construction industry (e.g. roofing applications), the failure mechanism is acknowledged as cutedge corrosion of the galvanic couple, leading to organic coating delamination. This enables the realisation that any improvement to the performance of the zinc alloy-steel couple will reap rewards in terms of product lifetime. Therefore, drive to consistently develop these alloy systems to maintain or extend their lifetimes is apparent. Whilst a prominent aim of this persistent development is to minimise production costs due to the highly competitive nature of the business, there is also motivation in light of impending REACH legislation. This EU-wide legislation prohibits the use of chromium(VI) compounds, which, compared to more commonplace alternatives, function as highly effective yet conversely toxic, carcinogenic, and mutagenic corrosion inhibitors; this is discussed further in 1.3.3.

The result of the above factors warrants the significant interest from steel manufacturers in ternary zinc-magnesium-aluminium, or ZMA, galvanising alloys. As discussed in Chapter 1, the development of this ternary alloy offers prospective improvements in corrosion resistance compared to more traditionally employed galvanising compositions. This has captured the attention of not only the construction market, but the automotive sector also. Previous research clearly demonstrated that minor ternary additions of magnesium had a negative impact on the corrosion resistance of $\mathrm{Zn}$-Al alloys at the cut-edge [52]. However, on the whole, studies utilising greater Mg additions have indicated enhanced levels of corrosion resistance in contrast with HDG under accelerated corrosion testing [61], [68], [70], [72], [73]. It is understood that corrosion resistance is highly influenced by microstructural effects, including factors such as the volume fraction of phases and their morphology [49], [82], [83]. It has been demonstrated that $M g Z n_{2}$ phase is attacked preferentially in a ZMA alloy during aqueous 
corrosion [78]. It is understood that amongst other phases, $M g Z n_{2}$ readily forms in these magnesium-containing alloys, increasingly so with higher $\mathrm{Mg}$ content [77]. This $\mathrm{MgZn}_{2}$ phase has also been realised to be a highly beneficial phase when used in conjunction with an organically-coated steel system [74]. However, the same study indicated that the highly reactive nature of this phase may not prove as beneficial for a Zn-Mg alloy system in the uncoated condition. The variation in microstructure for ZMA alloys provides ample reason as to why studies must consider the corrosion performance of different compositions, to suit the application in which they are used. Consequently, the aim of this chapter is to consider the microstructure, the corrosion performance, and related mechanisms according to changes in Mg and Al content.

This work explores three different ZMA-coated galvanising alloy compositions, the details of which are set out in Table 3.1. The microstructures of the differing alloys were examined using optical light microscopy, and scanning electron microscopy (SEM) coupled with energy-dispersive X-ray spectroscopy (EDS) for elemental analysis. The relative corrosion performance of each alloy was assessed using scanning vibrating electrode technique (SVET) to electrochemically map localised surface corrosion features, and in situ time-lapse optical microscopy to study corrosion on a microstructural level. A portion of this work has gone to publication (Appendix 1), and thus, some of those outlined understandings are also ventured here.

\subsection{Experimental}

\subsubsection{Metallic specimens}

The details of the differing ZMA alloys in question are displayed in Table 3.1. Each of the alloys are from commercial manufacturers, which were supplied via the French Corrosion Institute. These coated steel products were all produced under production line conditions.

Table 3.1 Composition of the three zinc-magnesium-aluminium alloy coatings of interest

\begin{tabular}{|c|c|c|c|c|}
\hline Sample & Zn (wt\%) & Mg (wt\%) & Al (wt\%) & $\begin{array}{c}\text { Coating thickness } \\
(\mu \mathrm{m})\end{array}$ \\
\hline ZMA 1 & 98 & 1 & 1 & 10 \\
\hline ZMA 2 & 96 & 2 & 2 & 10 \\
\hline ZMA 3 & 93.3 & 3 & 3.7 & 10 \\
\hline
\end{tabular}

3.2.2 Sample preparation

All samples were prepared as described in 2.1.2.1, whereby they were guillotined into ca. 20 $\mathrm{mm} \times 20 \mathrm{~mm}$ coupons, mounted, polished to a flat $1 \mu \mathrm{m}$ finish, and then cleaned with distilled 
water followed by ethanol. Microstructural features were revealed using a Nital etchant solution, proceeded by the same cleaning procedure. For investigation using SVET, further preparations involved taping the entirety of the sample surface with adhesive PTFE tape, except for an exposed $10 \mathrm{~mm} \times 10 \mathrm{~mm}$ approximate area of the surface. Time-lapse microscopy experiments followed a similar masking procedure, the difference being a circular feature of 1 $\mathrm{mm}$ diameter $\left(0.785 \mathrm{~mm}^{2}\right)$ surface was exposed in its place.

\subsubsection{Assessment of ZMA alloy corrosion performance and mechanisms}

SVET scans were performed on the range of alloy compositions in question as a method of determining the extent of corrosion activity occurring on the surface. This was achieved by not only mapping a visual representation of the surface corrosion events in terms of normal current density distributions, but through production of a semi-quantitative value for metal loss. All experiments performed on the three alloy types were conducted in a $1 \% \mathrm{w} / \mathrm{v}(0.172 \mathrm{M}) \mathrm{NaCl} \mathrm{pH}$ 7 solution, which for this work was considered as the baseline electrolyte; the samples were allowed to freely corrode over the 24-hour experimental time-frame. Samples were scanned at the onset of the experiment, and scanned hourly therein until the experiment time-frame was elapsed. Further details of the SVET apparatus and operation can be found in 2.2. Calibration of the SVET was performed preceding each experiment using the two-compartment cell described in 2.2.2.

To further assess the trio of alloy compositions being studied, a novel time-lapse microscopy technique was utilised. This technique, based on a previously developed system, enables in situ examination of features of corrosion activity at a microstructural level when immersed in a corrosive electrolyte. Each prepared sample was fitted onto the stage and then immersed into a $1 \% \mathrm{w} / \mathrm{v} \mathrm{NaCl} \mathrm{pH} 7$ electrolyte within the glass tank, and the microscope lens set into position ready for image capture. These images were taken successively in 2-minute intervals for the duration of the experiment. A more in-depth explanation of the setup is outlined in 2.3 .

\subsection{Results and discussion}

\subsubsection{Study of the microstructures of the ZMA alloys}

Microstructural images of the individual ZMA alloys were captured using optical light microscopy, as displayed in Figure 3.1. The images clearly showed a distinct primary dendritic phase surrounded by a eutectic matrix. As the alloying additions of $\mathrm{Mg}$ and $\mathrm{Al}$ increased, it was apparent that volume fraction of the different phases varied considerably. The dendritic phase, 
understood to be the primary zinc phase, exhibited a decrease in volume fraction coincident with an increase in alloying additions, similarly observed by Vlot et al. [77]. Consequently, a corresponding increase in the eutectic phase was evident. A substantial change in the morphology was also present. The profile of the primary dendrites underwent a marked refinement when considering the highest alloyed sample in comparison with that of ZMA 1 and 2. The sheer extent of overall refinement seen in Figure 3.1 C suggests a potentially higher cooling rate during the manufacturing process; however, this may simply be a phenomenon of the increased $\mathrm{Mg}$ and $\mathrm{Al}$ quantities.
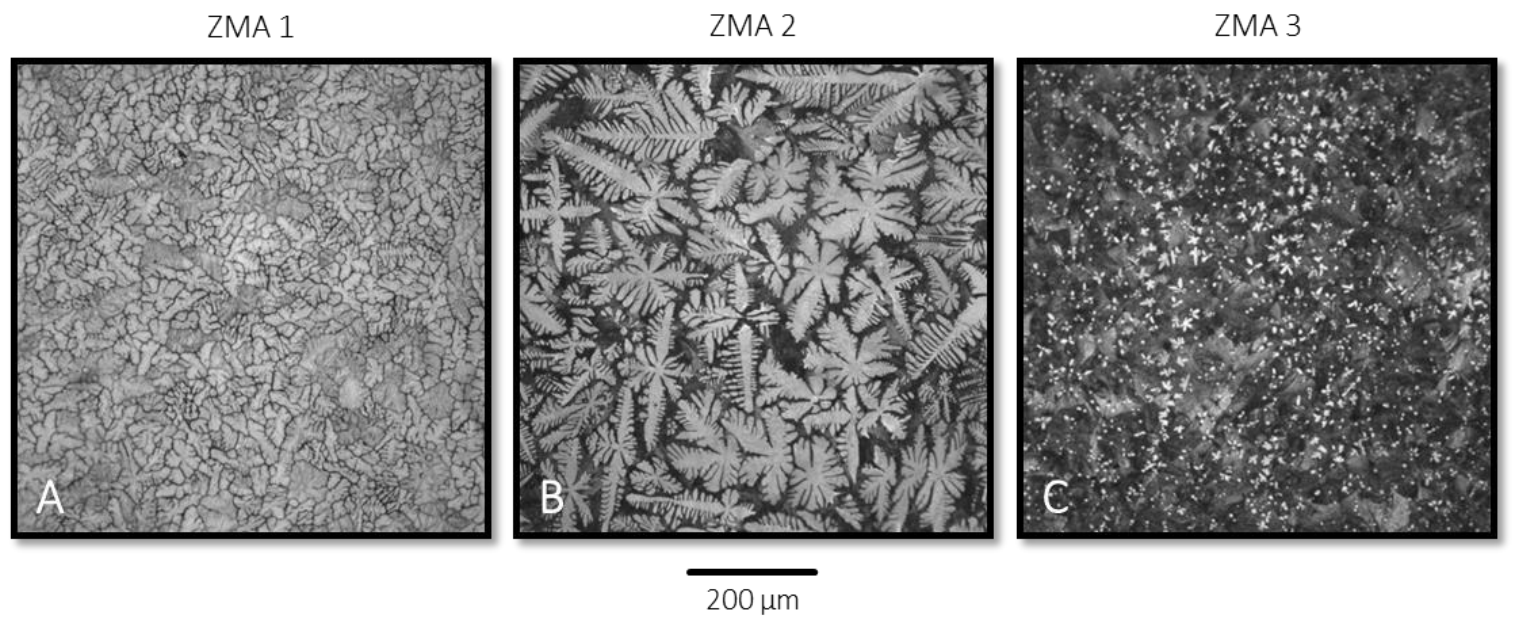

Figure 3.1 (A-C) Optical light microscope images of each ZMA alloy. All alloys encompass a microstructure of primary dendrites and eutectic phase

The optical light microscope images of each alloy were analysed to quantify the volume fraction of phases present as a percentage, as seen in Figure 3.2. The data highlights the substantial shift in the formation of phases occurring with change in alloy addition. The increasing amount of magnesium and aluminium in ZMA 2 and 3 comparative to the ZMA 1 sample, exhibited a sharp fall in primary $\mathrm{Zn}$ with a concurrent increase of the eutectic phases present. Contrasting ZMA alloys 1 and 3, the upturn in volume fraction of the eutectic phases from $18 \%$ to $91 \%$ respectively, is clear.

Previous work on this class of alloy demonstrated the formation of a primary zinc dendritic phase containing small amounts of $\mathrm{Al}$, and minute quantities of $\mathrm{Mg}$ [77]. The microstructure is understood to be accompanied by a binary and ternary lamellar eutectic, of which the binary is rich in zinc and magnesium, and the ternary composed of $M g Z n_{2}$ and $Z n$, with nodules of Al appearing in the ternary lamellae [72], [78]. Under production line conditions, these alloys likely undergo a rapid, non-equilibrium cooling, and as such, $\mathrm{MgZn}_{2}$ forms in preference to $\mathrm{Mg}_{2} \mathrm{Zn}_{11}$ as confirmed in other work [77]. To validate the current 
understanding of the ZMA microstructure, further imaging was completed in the form of scanning electron microscopy, coupled with energy dispersive X-ray spectroscopy (SEM-EDS). Figure 3.3 supported the presence of a dendritic primary zinc phase amongst a eutectic matrix in each sample. This dendritic phase is understood to be rich in zinc, containing minimal magnesium and a small amount of aluminium ( $<0.7 \mathrm{wt} . \%)$ according to the phase equilibrium diagram [58]. The occurrence of eutectic phases differing in morphology was definitive, where both a coarse and finer eutectic structure was present across the range of alloys. Other work considering ZMA-type alloys has found a binary eutectic comprising that of alternating Zn$\mathrm{MgZn}_{2}$ lamellar [60], and the ternary eutectic $\mathrm{Zn}, \mathrm{MgZn}_{2}$, and Al [78], [87]. The corresponding EDS results from Figure 3.4 verified the phase compositions described above, highlighting the presence of $\mathrm{Zn}$ and $\mathrm{MgZn} 2$ in the coarse and thus, binary eutectic. There was also fitting agreement that the finer eutectic was comprised of $\mathrm{Zn}, \mathrm{MgZn}_{2}$, and $\mathrm{Al}$, and consequently of ternary composition. At the higher magnifications achieved using SEM, the presence of Al-rich dendrites was also apparent, and increasingly so as the alloying content was increased. This was highlighted via the EDS mapping, and may be of similar composition to the $\mathrm{Zn}$-containing Al phase observed in previous work [77].

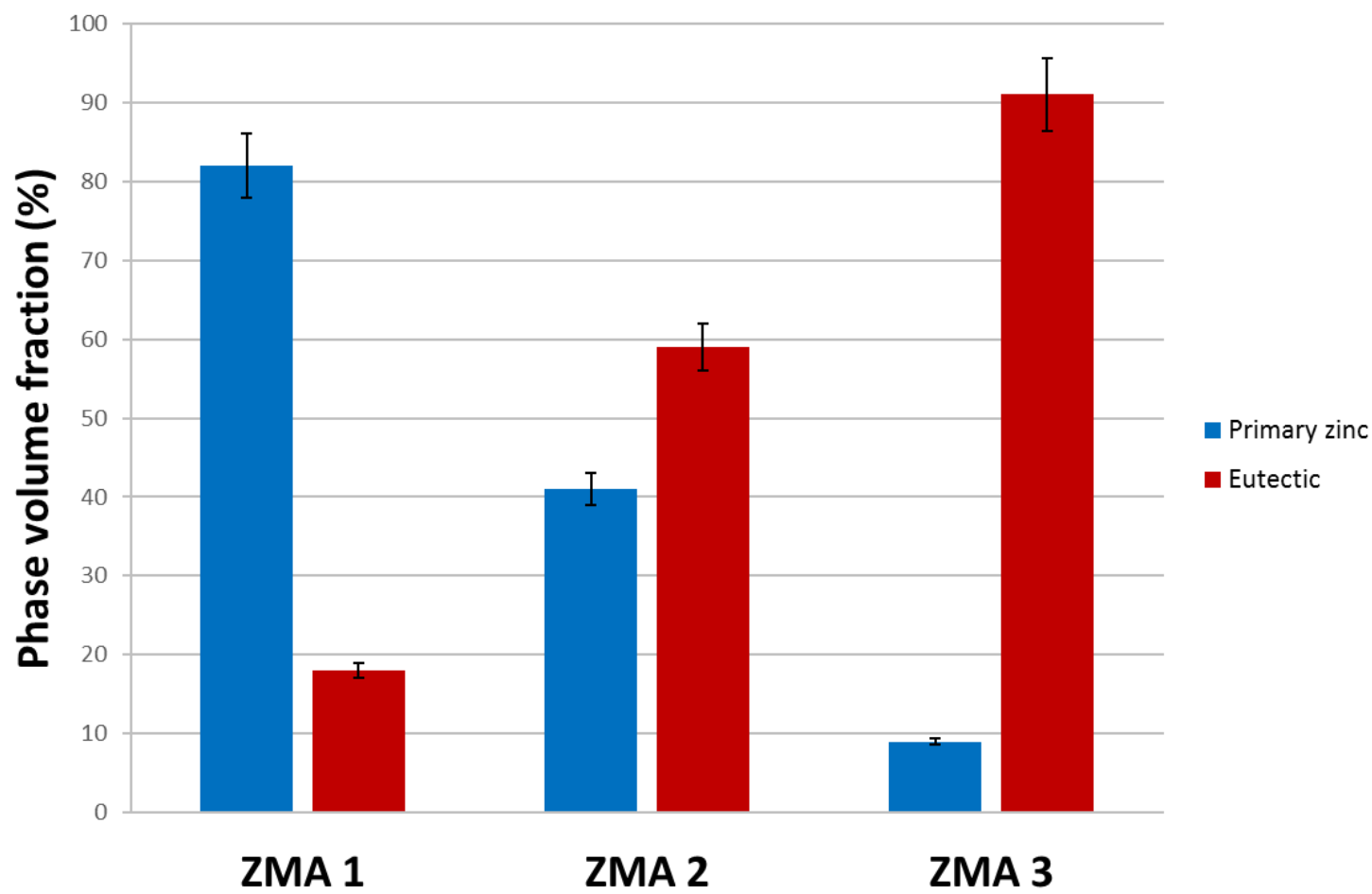

Figure 3.2 Volume fraction (\%) of microstructural phases present in the three ZMA alloy compositions 

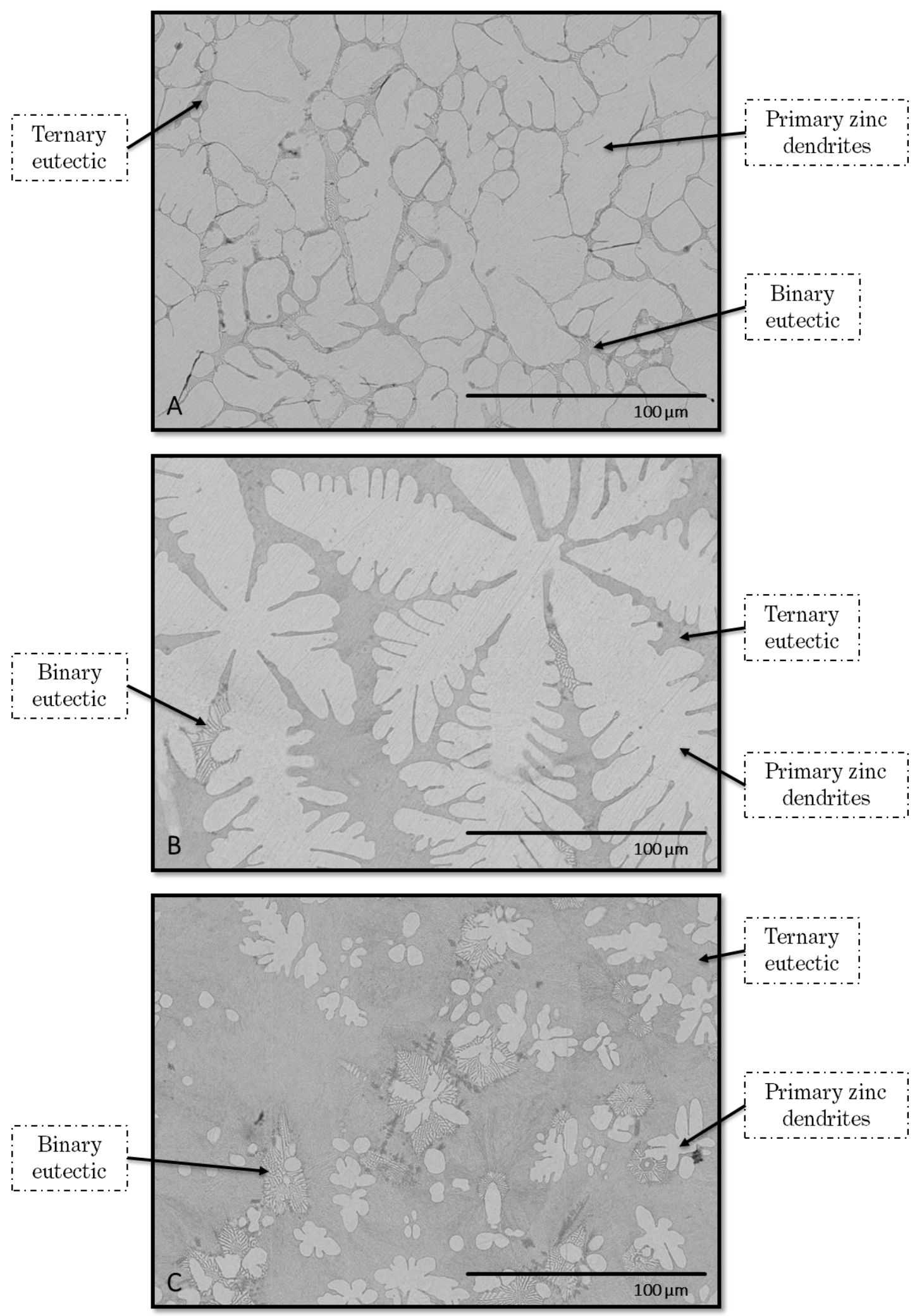

Figure 3.3 SEM images of A) ZMA 1, B) ZMA 2, and C) ZMA 3 samples 


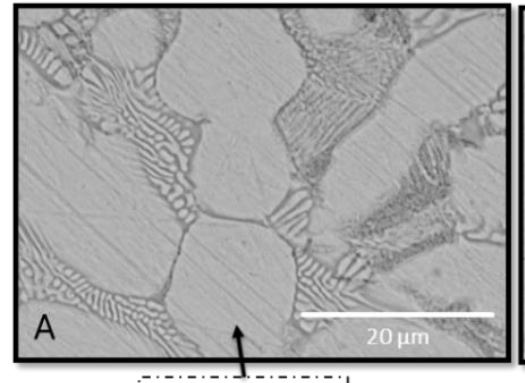

Primary zinc dendrite
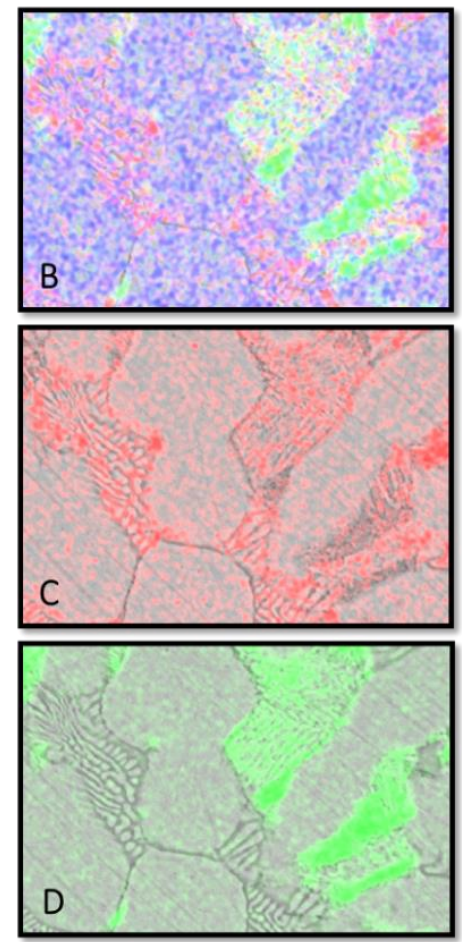
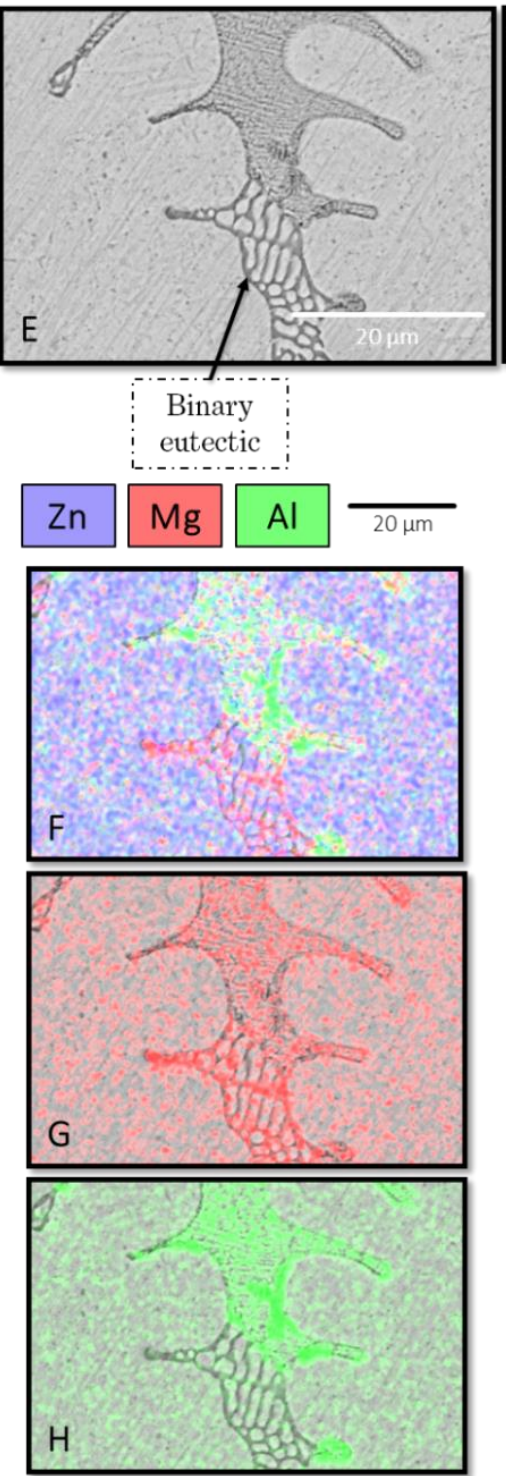

J
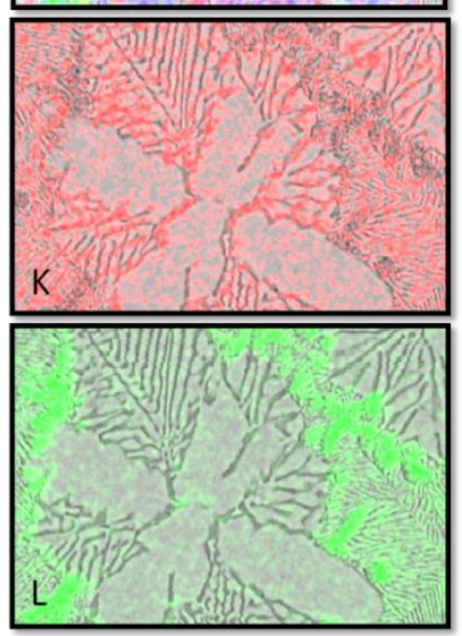

Figure 3.4 SEM-EDS analysis of: (A-D) ZMA 1, (E-H) ZMA 2, (I-L) ZMA 3

\subsubsection{Investigation of corrosion behaviour using SVET}

The SVET was used to study the surface corrosion events for the three ZMA alloys of interest in an aerated $1 \% \mathrm{w} / \mathrm{v} \mathrm{NaCl} \mathrm{pH} 7$ electrolyte. The resulting surface-resolved current density maps of the alloys have been plotted, in addition to the estimated values for metal loss based over the entirety of the experiment. The full explanation for the data calculations can be found in 2.2.5. As the principal element in each of the alloys, zinc was selected as that undergoing metal dissolution during the metal loss calculations. During corrosion of these alloys, both $\mathrm{Zn}^{2+}$ and $\mathrm{Mg}^{2+}$ ions are likely to be involved, and therefore, definitively stating which element governs these reactions with respect to time is problematic. However, due to the identical charge associated with each element to be used in the Faraday equation, a parallel relationship of performance would be achieved; selection of the other element (i.e. $\mathrm{Mg}^{2+}$ ) would only act to 
alter the overall metal loss value, but the fundamental relationship between each of the alloys would continue irrespective of element selection. For this reason, the SVET-derived metal loss values have been used as an indicator of corrosion performance between each of the ZMA alloys, keeping in mind that the values do not represent an exact metal loss value for the 24 hour experiment duration.

Figure 3.5 signifies the estimated values for metal loss for each of the ZMA alloys as determined by SVET; from this it was evident that the highest observable metal loss stems from the alloy containing the minimum additions of $\mathrm{Mg}$ and Al. The ZMA 2 alloy exhibited a fall in measured metal loss by $22 \%$ to that observed in ZMA 1, followed by ZMA 3 demonstrating a reduction of $39 \%$ compared to that of ZMA 1 . The results showed that an increase in levels of Mg and Al translated to a decline in measured metal loss. The distinct trend suggests a pronounced increase in corrosion performance within the higher alloyed samples.

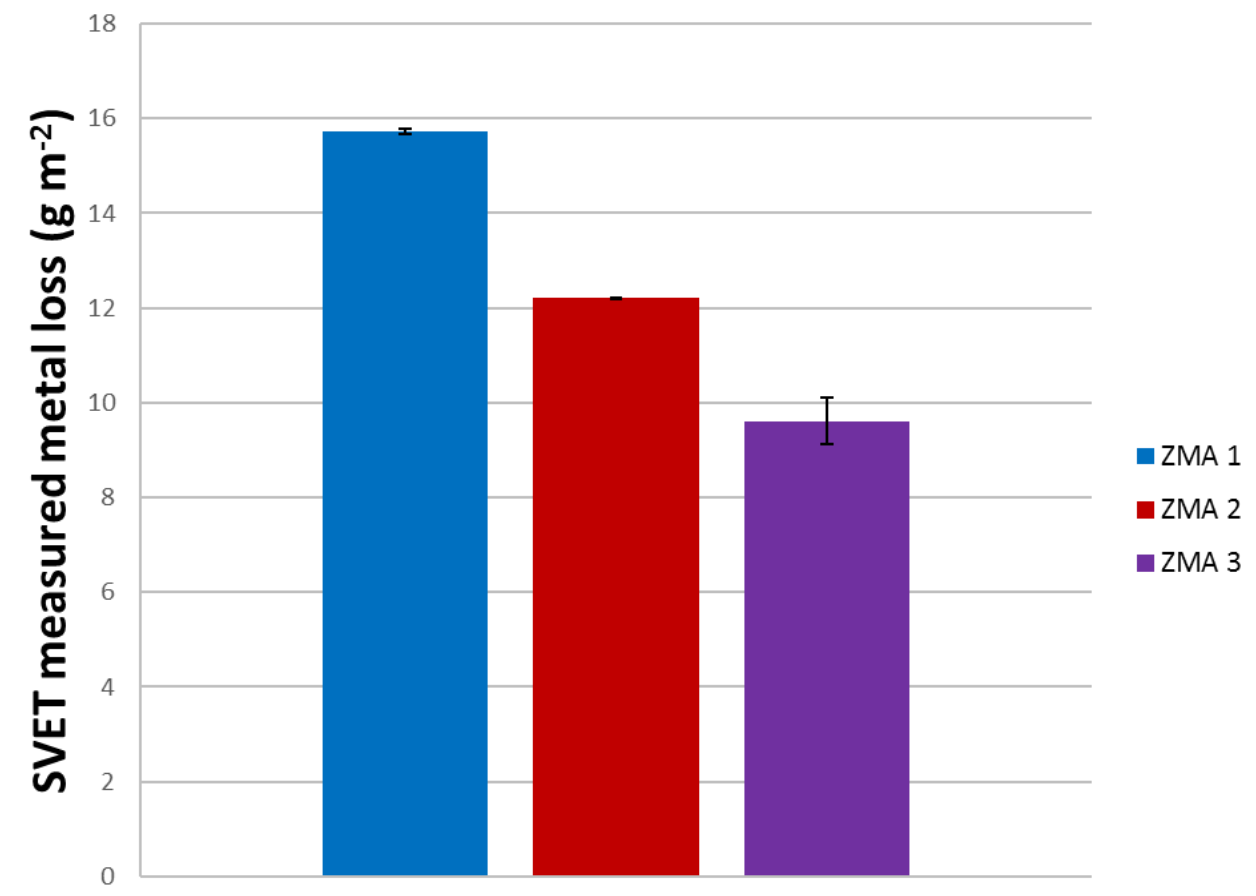

Alloy composition

Figure 3.5 SVET-derived metal loss over 24-hour experiment time for the ZMA alloy range studied in $1 \% \mathrm{NaCl}$ pH 7 solution

The SVET can provide further data in the form of spatially resolved maps of normal current density during each scan. Essentially, this provides a visual representation of the location and intensity of anodic and cathodic events, as registered by the SVET probe. The colour change from red to blue is indicative of the shift from anodic to cathodic activity. The current density contour maps for ZMA alloys 1-3 are portrayed in Figures 3.6-3.8. These include a selection of 
maps at set intervals over the duration of the experiment for the sake of brevity. Regarding all three ZMA alloys, the scan performed at hour 1, or that from the launch of the experiment, was omitted due to the minute amount of surface activity compared with the remainder of the scans. Figure 3.6 showed that a multitude of point anodes were apparent on the surface from hour 1 , seeming to form in clusters, which therein revealed a large portion of the remaining sample surface to be cathodic. Whilst the point anodes were still present by hour 4 , their profiles noticeably began to change, progressing outwards in a radial manner. Some of these anodes displayed signs of agglomeration, and despite this, continued to follow a radial pattern of progression. On the whole, the intensity of the anodes also diminished. Upon hour 8, the majority of the previously observed point anodes lessened in magnitude or de-activated towards the top half of the sample. The two remaining anodes also reduced in intensity, countered by the nearby formation of islands of fresh anodic activity, showing a comparatively high current density. The newer and more intense anodic activity displayed signs of passivation and lessened intensity by hour 12 . Interestingly, the area of the sample that had previously retained a passive quality had instead become overwhelmed with anodic sites, made up of both focal and island-like arrangements. Between hours 12-20, the larger anodic islands passivated for the most part, and numerous point anodes were prevalent on the surface elsewhere. Following this, the radially progressing anodes were observable once again, continuing to form island-like contours. Considering hours 20-24, the propensity for variation in surface activity was comparatively reduced, where less anodic profile evolution was visible than previously seen. However, the increase in intensity of cathodic activity was evident, focused predominantly on the interior void of the anodic islands. Furthermore, these cathodic 'cavities' were not apparent in every instance, appearing only within anodic islands of sufficient dimension. The reason for this activation of interior cathodic activity may be due to the presence of fresh substrate material consequent of the anodic attack.

The study of ZMA 2, shown in Figure 3.7, yielded similarities to that seen in ZMA 1, but in a less pronounced manner. Several localised point anodes were obvious following the hour 1 scan, which were of a reasonably high intensity as dictated by the limits of the scale bar. These anodic features persisted throughout the majority of the experiment, until hour 20 . The outlines of these enduring features were still evident at hour 24 , no longer acting anodically but having adopted passive properties instead. A recurrence of the radial anodic progression observed in Figure 3.6 was noticed between hours 4-20, developing from single points to ringlike features. This was in addition to the observed change in morphology, where larger ring-like attributes were established following coalescence of the smaller, focal anodes. The hollow 
pseudo-circular anodic features were realised to have a limited lifetime, slowly growing until a break was visible on the perimeter of the anodic ring; this, ultimately led to passivation of the feature, as visualised in hour 20 of Figure 3.7. Upon hour 20 of the experiment, islands of anodic activity developed akin to that noticed in the ZMA 1 alloy. Simultaneously, anodes active from the first hour either began to diminish in intensity or passivate, as evidenced from hours 20-24. The final scan displayed remnants of previously active anodes, together with newly developed anodic islands, some of which were of high current density. The island-like anodes arose mostly in regions of cathodic activity on the surface, demonstrating a transition from what was originally a site for cathodic activity to a more aggressive anodic one. Overall, the fluctuations in corrosion events observable from scan to scan seemed less prominent, and exhibited a more gradual variation in activity than previously noted in ZMA 1. Once again, cathodic activity within emanating anodic sites noted for ZMA 1 was also present during the experiment, however the magnitude of this activity was notably reduced.

The ZMA 3 alloy of Figure 3.8 displayed an onset of intense anodic activity upon examination of hour 1 . The cluster of anodes of mixed profile, yet again demonstrated outward progression in a radial fashion by the fourth hour. Amalgamation of some anodes was clear as the experiment proceeded from hours 8-16, where a larger anode formed with an irregular profile akin to a two-dimensional dendrite. Within that same time-frame, new anodes were also formed proximal to the existing larger feature. Whilst more anodes slowly formed between hours 16-24, mild cathodic activity was present in the expanding cavity of the larger anodic feature; this increased in intensity concurrent with the outward growth. As the cathode intensity increased on the inner region of the distinct anode, the anodic activity conversely reduced, potentially indicating imminent passivation of this feature. The cathodic activity was noticed to increase in intensity with progression of the experiment, while the cathodic wave swept across the surface gravitating towards the anodically dominated area. The evolution of corrosion events for ZMA 3 was less dramatic than that realised from the other two alloys, and there were fewer new anodes registered by the SVET, as visualised in Figure 3.8.

Considering Figure 3.7, the evolution of anodic activity was less marked on the whole in contrast to that shown in ZMA 1, but more so than visible in Figure 3.8. This was indicative of the corrosion kinetics per change in alloy composition. The ZMA 2 SVET maps showed that the positive-going current density features possessed lifetimes that endured the majority of the experiment, regardless of the observable differences in profile. However, ZMA 3 exhibited less aggressive anodic activity than observed for both other alloys. Reflecting on the differences between all three ZMA samples, the quantity of surface area occupied by anodes maintained an 
inverse relationship with regard to measure of alloy content; the highest alloyed sample displayed the smallest extent of anodic coverage on the surface. Accordingly, it was also the case that the higher content of $\mathrm{Mg}$ and $\mathrm{Al}$ present lowered the magnitude of current density values registered by SVET. Additionally, SVET anode analysis given in Figure 3.9, aided this to some extent; the plot demonstrated ZMA 1 to offer a varied rate of growth over the course of the experiment. The ZMA 2 anode growth was more stable than for ZMA 1 and reached a similar size before the selected ZMA 2 site was no longer active. The ZMA 3 anode size in fact suggested that this alloy enabled the most aggressive attack, however this was not the case. The ZMA 3 sample exhibited coalescence of multiple anodic sites, whilst supporting fewer sites of lower intensity overall. This was similarly the case for the ZMA 2 alloy, possessing fewer anodic sites of a lessened intensity than ZMA 1 . This creates the realisation that the increase in alloy content manifests as a corresponding increase in corrosion performance, and fully corroborates with the estimated metal loss results of Figure 3.5 .

The anodic current density averaged over an area $\left(\mathrm{Ja}_{\mathrm{t}}\right)$ for a given scan as measured by SVET was plotted in Figure 3.10. This considered the three ZMA alloys to envisage the progression of anodic current over the experimental time-frame, which can be related to the SVET contour maps as given in Figures 3.6-3.8. Figure 3.10 shows that both ZMA 1 and 2 exhibited a stark increase in $\mathrm{Ja}_{\mathrm{t}}$ from the beginning of the experiment up until the first hour. The prompt increase in $\mathrm{Ja}_{\mathrm{t}}$ value was curtailed from this point, and showed a more gradual escalation with time. The ZMA 2 alloy demonstrated a more moderate change in anodic current density from hour 1, agreeing with the stable progression of local anodes visible in Figure 3.7. The $\mathrm{Ja}_{\mathrm{t}}$ values for ZMA 1 undulated to a much greater extent than seen in ZMA 2, with the largest peak at hour 14 coinciding with the observed changes in anodic events between hours 12-16 in Figure 3.6. The ZMA 3 alloy displayed a higher Jat relative to the other samples from the onset of the experiment, which paralleled the intensity of the anodic features shown in hour 1 of Figure 3.8. The stable and gradual fall apparent in the values for ZMA 3 similarly conformed to the visual representation of the SVET maps. The plotted Ja data was derived from surface events observed in the SVET contour plots and in that sense, are parallel; however, they still provide useful means of drawing a relationship between both visual representation and data plot. The plots also confirmed the same noticeable trend, showing an enhanced corrosion performance via reduced overall Jat value for the highest alloyed sample. 

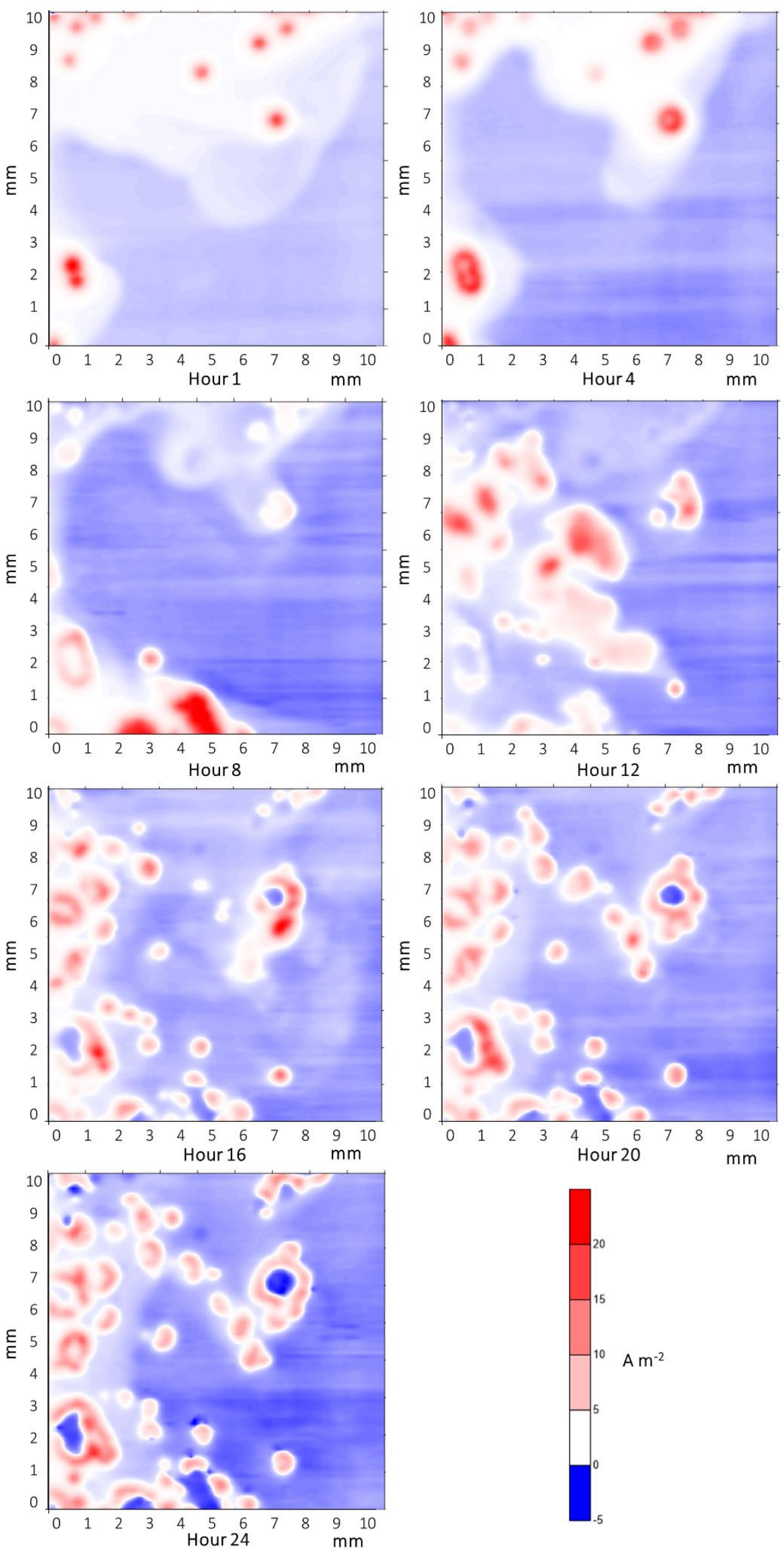

Figure 3.6 SVET-measured current density maps of ZMA 1 in $1 \% \mathrm{NaCl}$ pH 7 electrolyte 

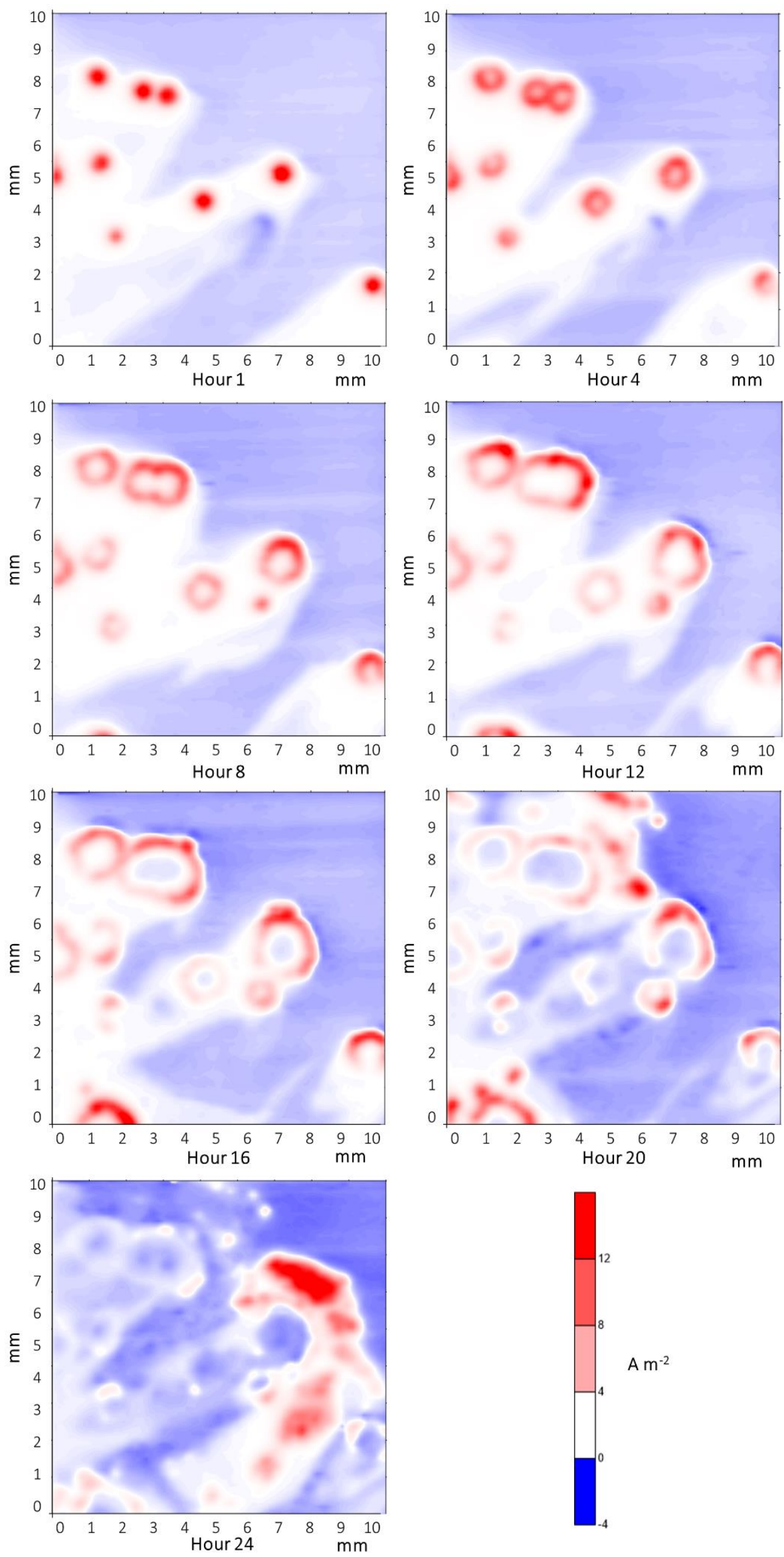

Figure 3.7 SVET-measured current density maps of ZMA 2 in $1 \% \mathrm{NaCl} \mathrm{pH} 7$ electrolyte 

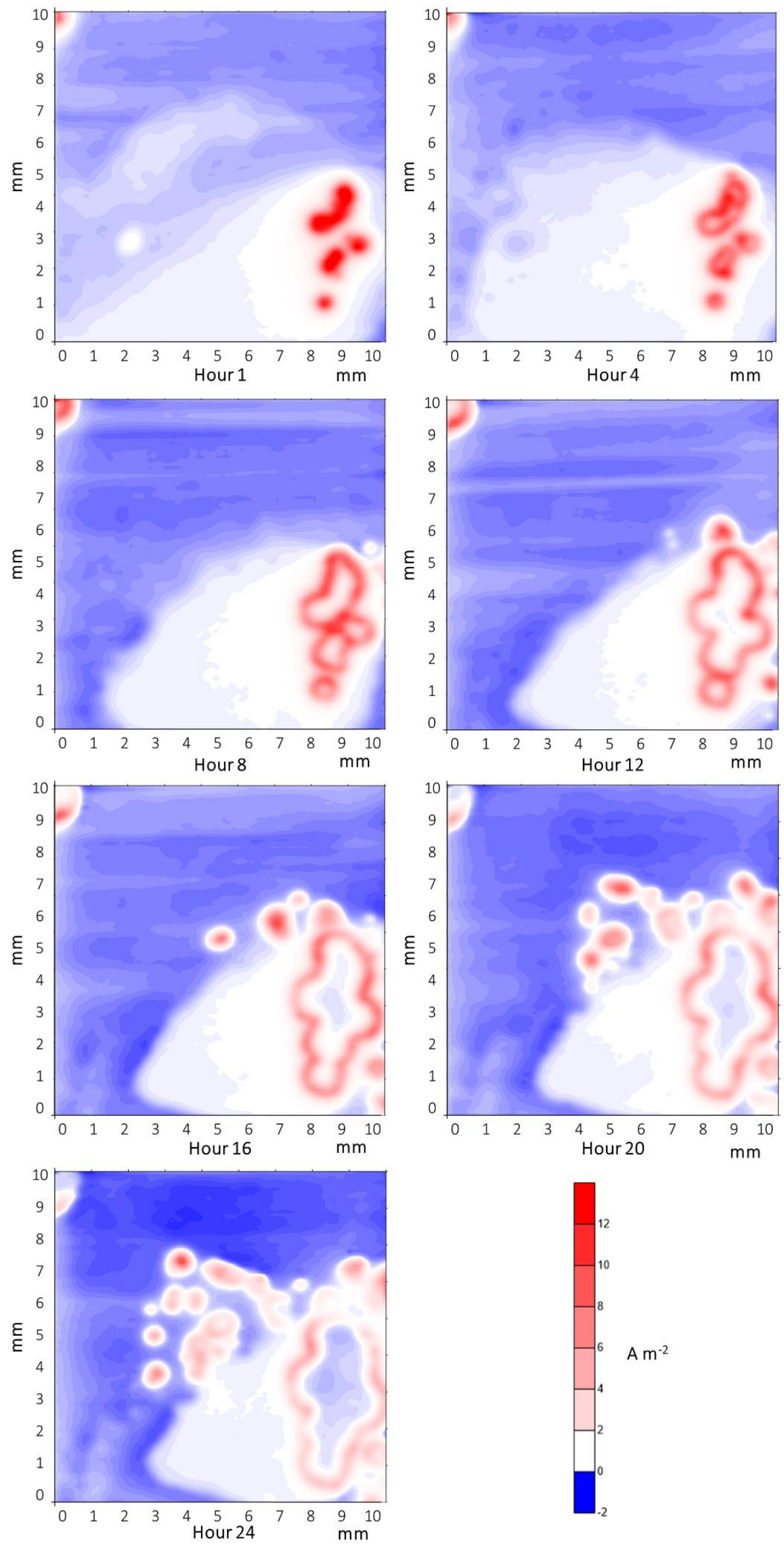

Figure 3.8 SVET-measured current density maps of ZMA 3 in $1 \% \mathrm{NaCl} \mathrm{pH} 7$ electrolyte 


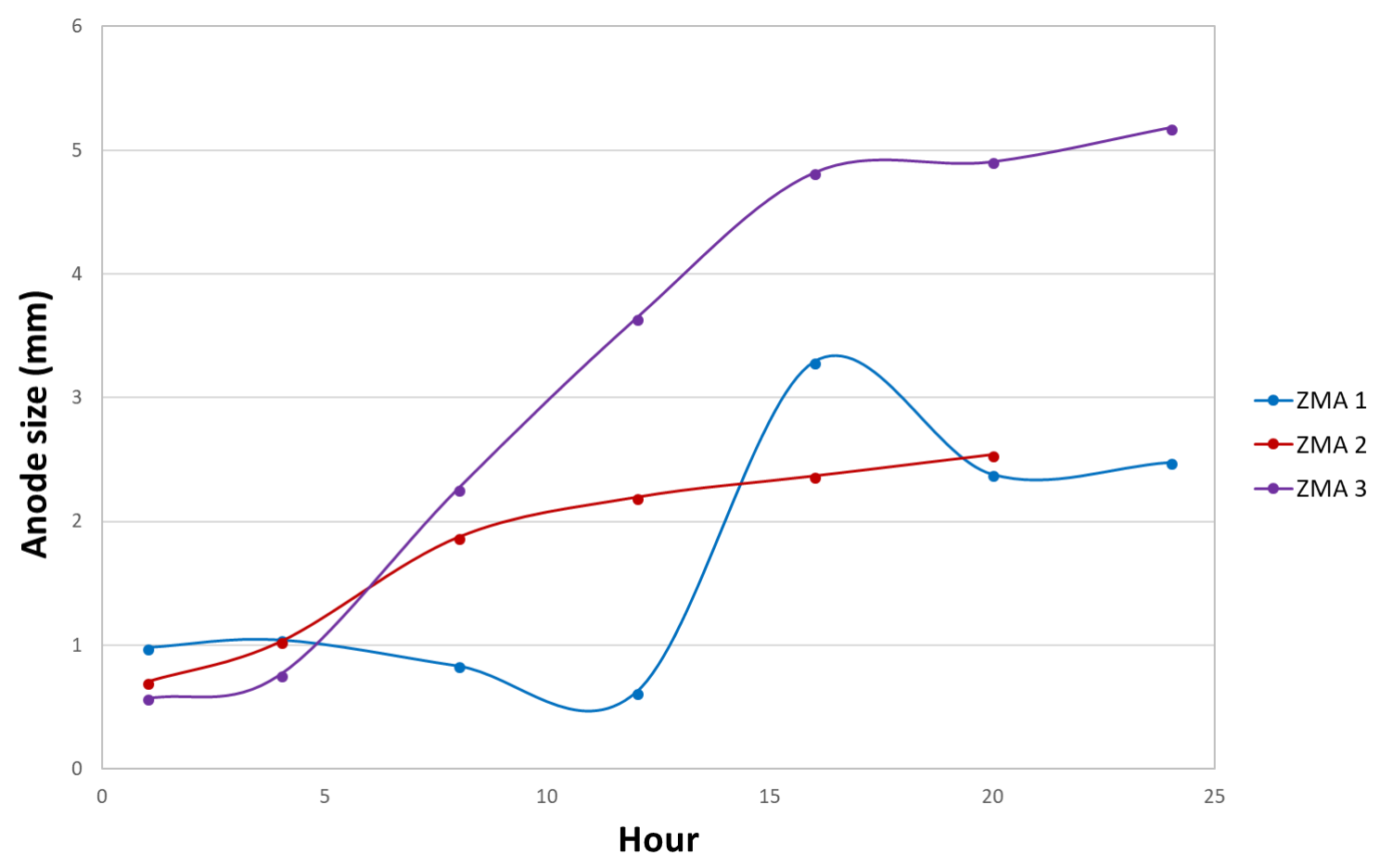

Figure 3.9 Plot of SVET anode size as a function of time

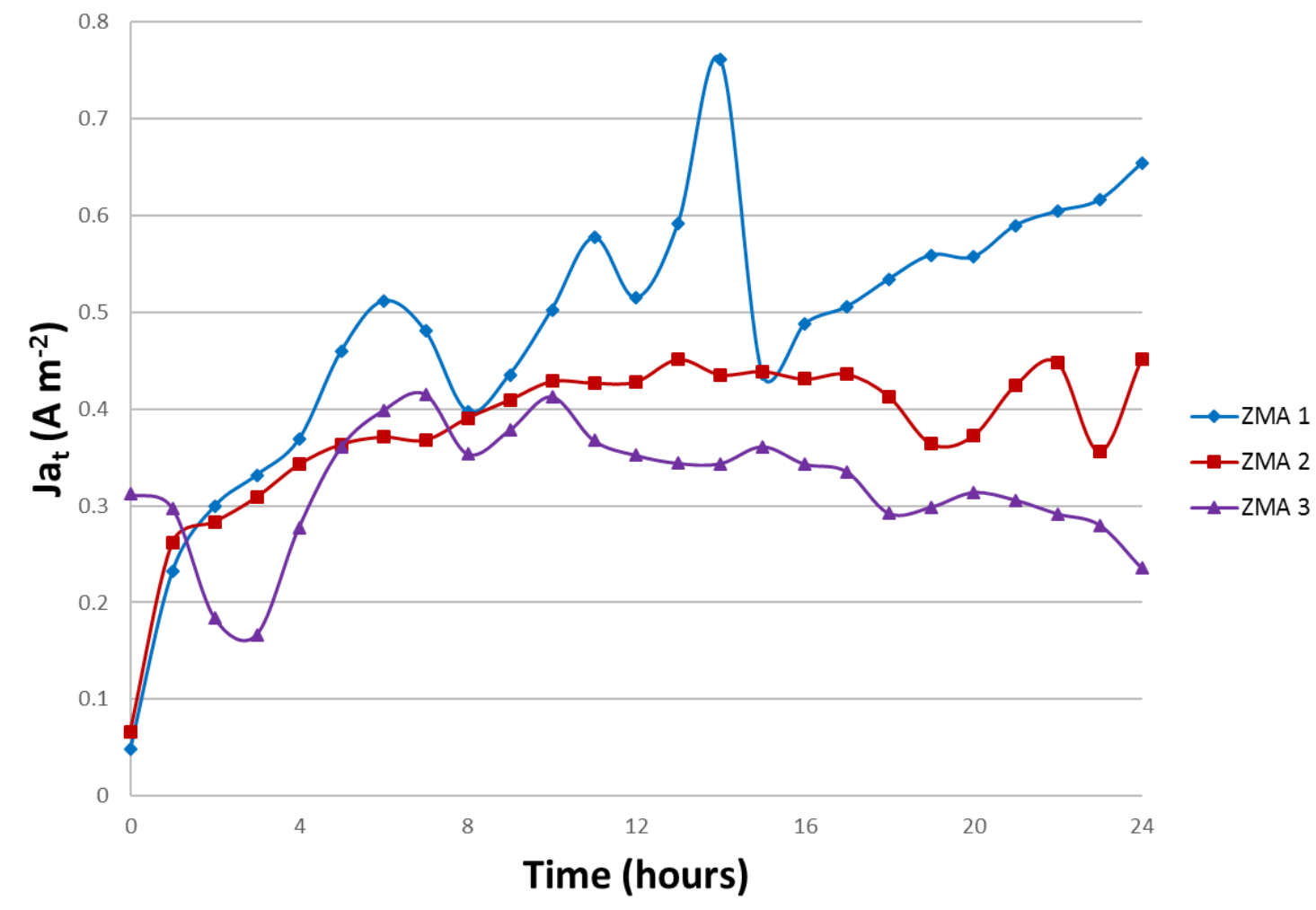

Figure 3.10 The area-averaged anodic-going current density $\left(\mathrm{J} \mathrm{at}_{\mathrm{t}}\right)$ plotted as a function of time for the ZMA alloy range in $1 \% \mathrm{NaCl}$ pH 7 electrolyte 


\subsubsection{Examination of ZMA alloy corrosion using time-lapse microscopy}

Figure 3.11 features the microstructures of each ZMA alloy and the associated corrosion mechanisms when under immersion conditions. Digital images were captured from the point of electrolyte introduction at two-minute intervals. In the case of Figure 3.11, it features the images captured at 2-hour intervals. Considering ZMA 1 of Figure 3.11 A-D, the initial image showed little visible corrosion activity occurring on the surface. However, the interval that follows demonstrated a definite anodic feature on the lower portion of the exposed surface. A corrosion product proceeded to deposit on the sample surface at the periphery of the anodic front, and precipitation of this corrosion product ring continued with progressive growth of the anode. It was worth noting that the discernible boundary seen in Figure $3.11 \mathrm{~B}$ between the anodic front and corrosion product was indicative of a transition from anodic to cathodic activity on the surface. Proceeding through each time interval, it was apparent that corrosion progressed through toward the midpoint of the sample, as elaborated on in the corresponding Video 3.1. It was observed that the progression of anodic activity preferentially followed the eutectic regions of the microstructure in the first instance, followed by attack of the primary zinc phase. The corrosion product ring was seen to migrate with respect to the anode, whereby portions of the ring dissolved and once again deposited on the surface at a given distance from the progressing anode. This dynamic movement of the corrosion product is suggestive of a decrease in $\mathrm{pH}$ local to the site of metal dissolution (i.e. the anode), which could cause such corrosion products to become soluble in solution once again. A similar result was observed for ZMA 2, which demonstrated a distinct and progressive anodic site accompanied by a ring of corrosion product proximal to the site, as shown in Figure 3.11 E-H. The time-lapse images for both ZMA 1 and 2 showed that anodic attack was focused preferentially on the eutectic phases, although this was much more apparent for the ZMA 2 sample. The anodic site originated in the eutectic and this activity was sustained in these phases, avoiding the primary zinc dendrites for some time. Video 3.2 proceeded to show that after approximately 4 hours, progression of corrosion events led to the eventuality of anodic attack on the primary phase. The ZMA 3 alloy, as shown in Figure $3.11 \mathrm{I}-\mathrm{L}$, experienced a noticeably reduced number and rate of progression of corrosion events in contrast to that seen with ZMA 1 and 2. Onset of visible corrosion activity was not established until the first hour, at which point activity commenced largely in the same manner as observed for the other alloys. The anodic attack predominated in the eutectic, seemingly avoiding the primary zinc-rich phase until the anode reached a critical size. A more extensive appreciation of the surface events is provided in Video 3.3. 
The time-lapse images were used to plot the total corroded area over time, extracted from the area of anodic attack, for a given time interval. This analysis was completed at 1-hour intervals over a total of 17 hours to plot the extent of anodic evolution with respect to alloy composition, depicted in Figure 3.12. This data assumes that excessive vertical pitting of the sample did not occur, and that corrosion instead progressed laterally across the sample surface; this is a restriction of using this two-dimensional technique. Using Photoshop CS6, the regions of anodic attack were selected and these areas were subsequently measured. The measurements were calibrated on a pixel-to-distance ratio by an image of known area, and an identical resolution and magnification to those of the time-lapse images. Figure 3.12 demonstrated a palpable trend, exhibiting the differences observed in extent of anodic evolution according to the different ZMA alloys of interest. The degree of lateral anodic progression coincided with a decrease in $\mathrm{Mg}$ and $\mathrm{Al}$ content for the alloy set, firmly agreeing with the data set from time-lapse microscopy and SVET experimentation.

The corrosion performance measured between SVET and time-lapse microscopy data sets indicated an equivalent trend, although the extent of the observable performance differences was not strictly proportional between each technique. By design, the SVET measures ionic current flux in solution emergent from the sample surface, whereas time-lapse microscopy considers only that envisaged as a two-dimensional area; therefore, the nature of the apparatus may permit the observation of corrosion activity, but to a less significant degree. 

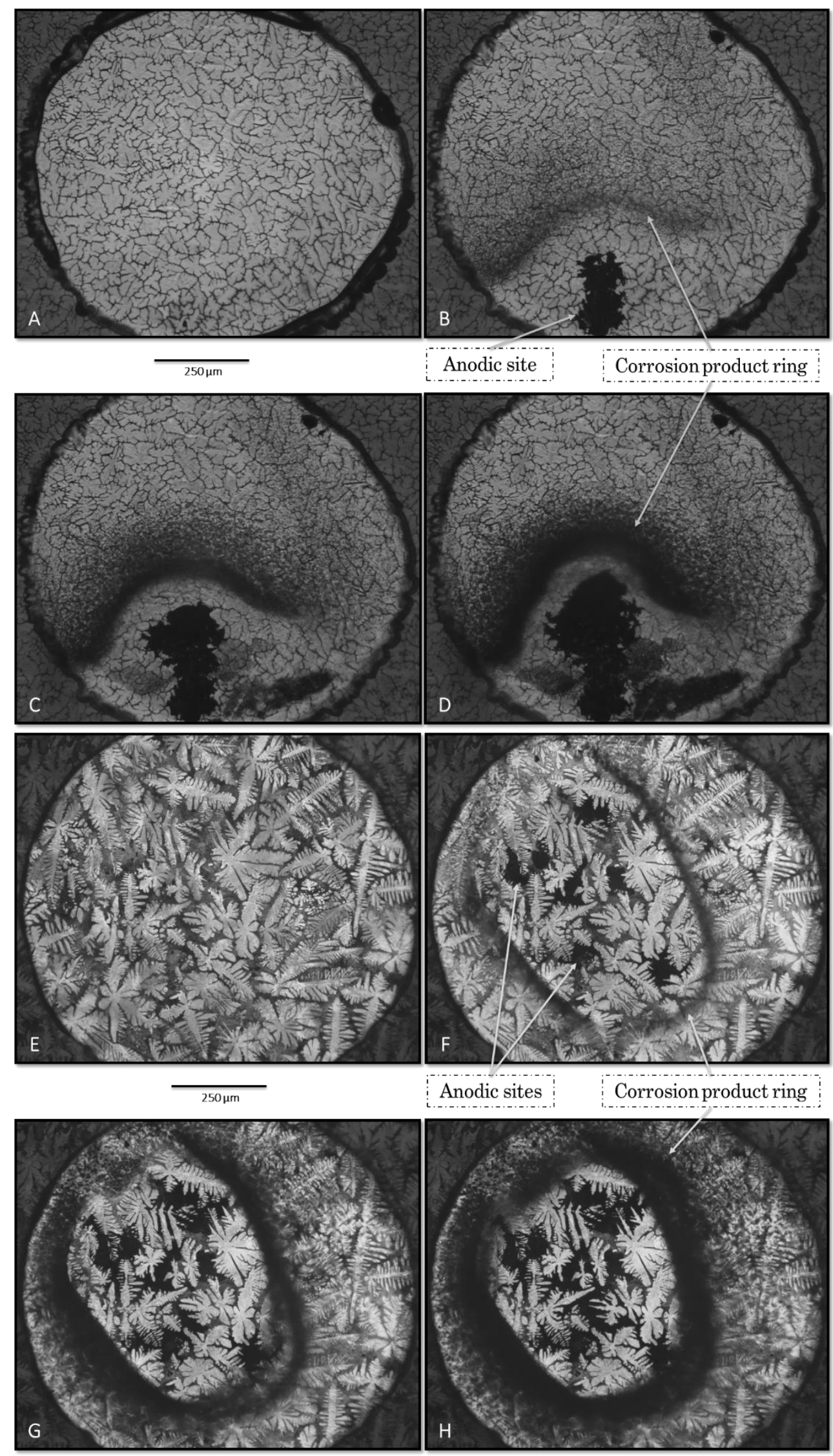

Figure 3.11 Time-lapse microscopy images taken at intervals of 2 hours in a baseline $1 \% \mathrm{NaCl}$ electrolyte, for: (A-D) ZMA 1, (E-H) ZMA 2, (I-L) ZMA 3 


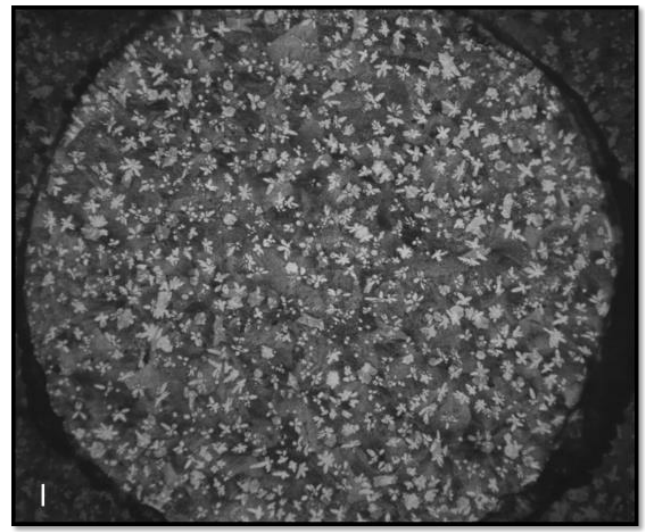

$\overline{250 \mu \mathrm{m}}$

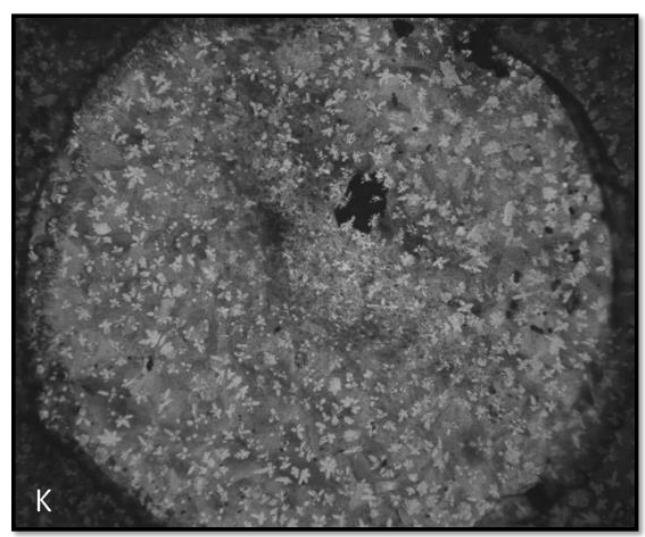

Corrosion product ring $\quad$ Anodic site

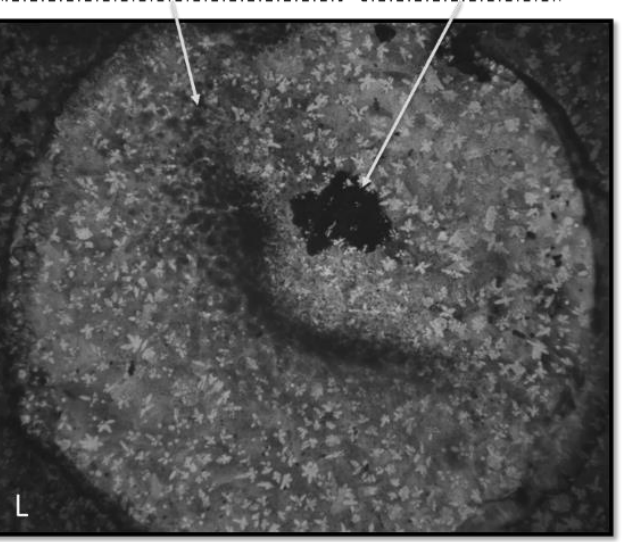

Figure 3.11 Continued

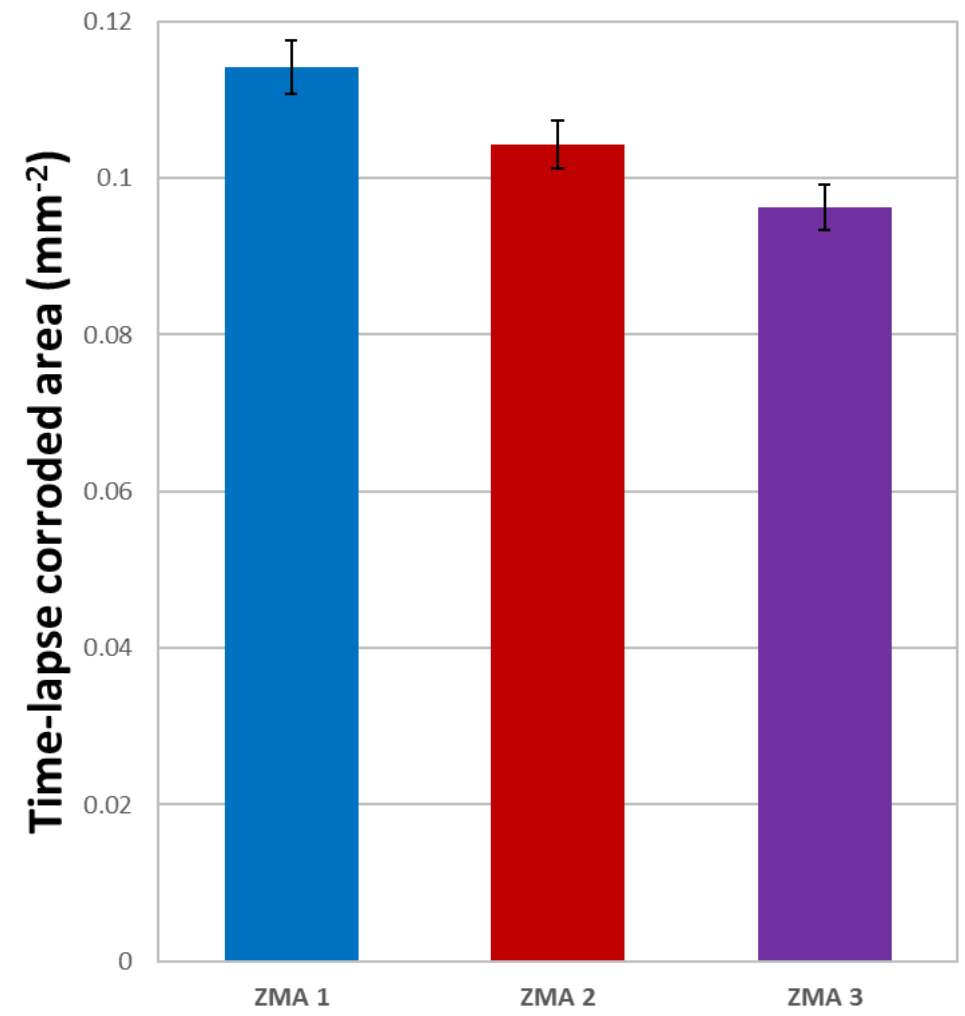

Figure 3.12 Total corroded area as measured by time-lapse microscopy for each ZMA alloy 


\subsubsection{Examination of corrosion behaviour of ZMA alloys according to alloy content}

variation

As discussed above, all SVET-derived data exhibited a distinct trend. This data showed that estimated metal loss values reduced according to an increase in both $\mathrm{Mg}$ and $\mathrm{Al}$ content within the zinc alloy range. The SVET current density maps also represented this correlation, where increasing the alloy content acted to reduce the extent of evolution in anodic attack. This was visually represented by the lessened degree of change in anodic profile between the ZMA alloys. The number of individual anodic sites also diminished following the increased quantity of $\mathrm{Mg}$ and $\mathrm{Al}$ addition. These SVET maps also revealed a characteristic evolution for all three ZMA samples, originating as point anodes and progressing outward in a somewhat radial manner, into ring anodes. The SVET performance trend was echoed by that of the time-lapse microscopy results (Figure 3.11), which displayed a parallel ranking between each of the three alloys. The corresponding plot of total corroded area according to each alloy, Figure 3.12, reaffirmed this trend. The time-lapse results evidenced the initiation of corrosion events in the eutectic, whereby these phases were preferentially attacked. Only once corrosion was sufficiently established in these regions did the attack then progress to the primary Zn phase. The extent of anodic progression diminished with increasing alloy content, indicating reduction in corrosion kinetics; this was most notable for ZMA 3 which took several hours to realise an appreciable growth of the anodic site.

As a means of verification, higher magnification SEM images were captured to further clarify sites of preferential attack on the ZMA microstructure. Samples were prepared to the same standard as for time-lapse microscopy experimentation discussed in section 3.2, revealing a circular area of $0.785 \mathrm{~mm}^{2}$. These samples were immersed in the baseline $1 \% \mathrm{NaCl}$ solution for 4 hours, and then removed to examine the surface using SEM. Figure 3.13 A clearly depicts that corrosive attack was focused on areas of eutectic, leaving the surrounding primary Zn phases intact. The higher magnification image of Figure 3.13 B more specifically illustrated the attack on the binary eutectic, showing that $\mathrm{MgZn}_{2}$ had been preferentially dissolved and the $\mathrm{Zn}$ lamellar remained. This corroborates research indicating that $\mathrm{MgZn}_{2}$ is attacked in the first instance on similar alloy compositions, acting as the less noble phase [47], [67], [78]. These results endorse the understanding that the higher reactivity of magnesium encourages corrosive attack of magnesium-containing phases in preference to zinc, within a ZMA microstructure. The associated Mg-containing corrosion products are purported to form surface films, providing ZMA alloys with a greater degree of corrosion resistance comparative to more traditionally employed compositions [71]-[73]. It has been suggested by Persson et al. that 
rapid dissolution of Mg-containing phases and the ensuing corrosion product is not beneficial exclusively during the initial stages of corrosion; the author indicated that ensuing corrosion product formation may be influenced during longer term exposure [73]. Although, such advantageous effects in these latter stages have not been confirmed.

$$
Z n \rightarrow Z n^{2+}+2 e^{-}
$$

$$
M g \rightarrow M g^{2+}+2 e^{-}
$$

$$
\mathrm{O}_{2}+2 \mathrm{H}_{2} \mathrm{O}+4 e^{-} \rightarrow 4 \mathrm{OH}^{-}
$$

Based upon the observations, it can be understood that the anodic reactions likely corresponded to Equations 3.1-3.2, whilst Al in turn supported the cathodic half reaction of Equation 3.3. The progression of corrosion under these conditions could potentially lead to the formation of several corrosion product species; this includes the formation of zinc hydroxide, magnesium hydroxide, hydrozincite, and simonkolleite according to Equations 3.4-3.7, respectively. Given that layered double hydroxides containing Al have been found experimentally in the study of various ZMA-type alloys [76], it seems likely that there is a breakdown in the passive $\mathrm{Al}_{2} \mathrm{O}_{3}$ layers despite the suggestion via the Pourbaix diagram in Figure 1.7. The Pourbaix diagram provides a thermodynamic indication of the state of a metal, however it may not offer a true reflection of the corrosion events. The ZMA alloys are made up of several different phases which contain varying quantities of each constituent metal, and relying on the predicted state for a single metal may not be as straightforward. Additionally, the electrolyte in question is that of a chloride-containing solution, whereas the Pourbaix diagrams considered in this work are for immersion in chloride-free media. Accordingly, Equation 3.8 may occur, thereby allowing the formation of layered double hydroxides given in Equations 3.9-3.10.

$$
\begin{gathered}
\mathrm{Zn}^{2+}+2 \mathrm{OH}^{-} \rightarrow \mathrm{Zn}(\mathrm{OH})_{2} \\
\mathrm{Mg}^{2+}+2 \mathrm{OH}^{-} \rightarrow \mathrm{Mg}(\mathrm{OH})_{2}
\end{gathered}
$$




$$
5 \mathrm{Zn}^{2+}+6 \mathrm{OH}^{-}+2 \mathrm{CO}_{3}^{2-} \rightarrow \mathrm{Zn}_{5}(\mathrm{OH})_{6}\left(\mathrm{CO}_{3}\right)_{2}
$$

$$
5 \mathrm{Zn}^{2+}+8 \mathrm{OH}^{-}+2 \mathrm{Cl}^{-} \rightarrow \mathrm{Zn}_{5}(\mathrm{OH})_{8} \mathrm{Cl}_{2}
$$

$$
\mathrm{Al}_{2} \mathrm{O}_{3}+3 \mathrm{H}_{2} \mathrm{O}+2 \mathrm{OH}^{-} \rightarrow 2 \mathrm{Al}(\mathrm{OH})_{4}^{-}
$$

$$
\begin{aligned}
& 2 \mathrm{Al}(\mathrm{OH})_{4}^{-}+6 \mathrm{Zn}^{2+}+8 \mathrm{OH}^{-}+\mathrm{CO}_{3}^{2-} \rightarrow \mathrm{Zn}_{6} \mathrm{Al}_{2}(\mathrm{OH})_{16} \mathrm{CO}_{3} \\
& 2 \mathrm{Al}(\mathrm{OH})_{4}^{-}+6 \mathrm{Mg}^{2+}+8 \mathrm{OH}^{-}+\mathrm{CO}_{3}^{2-} \rightarrow \mathrm{Mg}_{6} \mathrm{Al}_{2}(\mathrm{OH})_{16} \mathrm{CO}_{3}
\end{aligned}
$$

Considering once more the volume fraction of phases between the alloy set, given in Figure 3.2, the eutectic phase volume fraction increased in tandem with $\mathrm{Mg}$ and $\mathrm{Al}$ content. Understanding that the eutectic is attacked favourably, it could be assumed that the extent of corrosion would simply increase as a by-product of this preferential attack. However, the opposite of this statement was true. The cumulative alloy content instead improved the corrosion resistance, which cements the premise of favourable corrosion product formation. The presence and reactive nature of $\mathrm{Mg}$ and $\mathrm{Al}$, and the production of their relevant oxide layers, can be recognised as constructive for means of corrosion resistance. Whilst $\mathrm{Al}$ is more reactive than $\mathrm{Zn}$ according to the electrochemical series, it is realised that passive $\mathrm{Al}_{2} \mathrm{O}_{3}$ layers positively influence corrosion behaviour and are essentially inert for the cathodic oxygen reduction reaction [88], [89]. Therefore, an increase in Al content in a $\mathrm{Zn}$ alloy can explain, in part, the improvements observed in corrosion performance from the current data set [67]. 

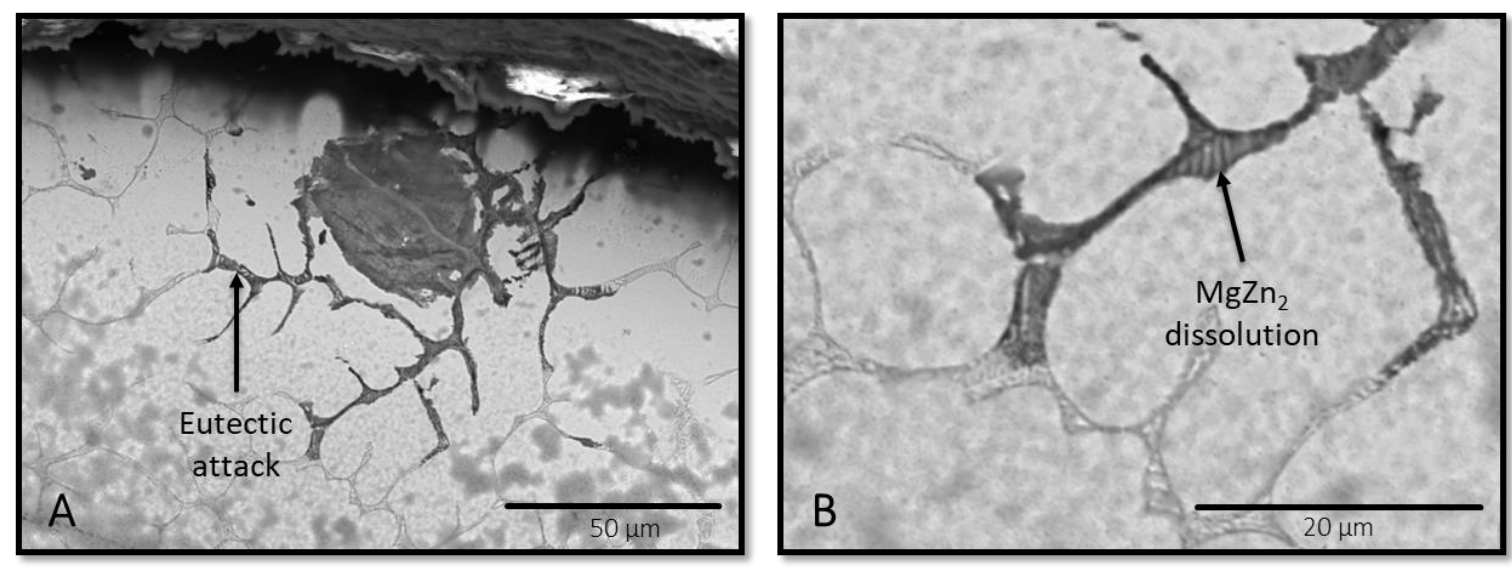

Figure 3.13 Microstructural examination of ZMA 1 using SEM, following 4 hours immersion time in $1 \% \mathrm{NaCl}$ electrolyte

The reactive nature of $\mathrm{Mg}$ would likely generate oxide layers on the surface of the ZMA alloys in question. Work by Hausbrand et al. showed that the native oxide layer in the $\mathrm{MgZn}_{2}$ phase is a Mg-rich passive oxide; the author stipulated that the semiconducting properties of the Mg-rich oxide is of a large bandgap [90]. This understanding was endorsed in other work, explaining that MgO having a larger bandgap than $\mathrm{ZnO}$ acted to significantly decrease the rate of cathodic reaction [91]; the larger bandgap corresponds to a lessened ability to transfer electrons from a metal, thereby reducing efficiency of oxygen reduction at the surface and contributing to improvements in corrosion resistance levels [71]. Further work by Prosek et al. found that the presence of Mg-based corrosion products hindered zincite ( $\mathrm{ZnO}$ ) formation [72], contributing to the favourable corrosion properties of ZMA alloys. The mechanism responsible has been proposed as a buffering effect of magnesium at higher $\mathrm{pH}$, inhibiting formation of $\mathrm{ZnO}$ and forming other favourable corrosion product species [47], [65], [72]. It has been documented that corrosion of zinc is largely stabilised in the presence of $\mathrm{CO}_{2}$ under conditions of atmospheric corrosion [92]. Similarly, LeBozec et al. demonstrated that the beneficial corrosion properties of ZMA comparative to other galvanising alloys were markedly reduced when in $\mathrm{CO}_{2}$ depleted environments [70]. The reaction of $\mathrm{Mg}$ with carbonate species was shown to be preferential over that of Zn [47], [66], which aligns with such work. Surplus to these mechanisms, Mg-based compounds in the form of magnesium carbonates or hydroxides have been proposed to provide direct protection by forming at the coating-corrosion product interface [71], [72]. Some authors have also postulated that ZMA offers improved protection capabilities due to the formation of simonkolleite [47], [65]. However, work instead revealed that hydrozincite and carbonate-containing compounds dominated the corrosion products under model atmospheric conditions [71], [72]. LeBozec et al. in fact concluded that the corrosion performance of ZMA cannot be explained entirely on the basis of specific corrosion 
product formation, which instead is most likely dependant on exposure conditions [93]. It was proposed that even the ratio of $\mathrm{Mg}$ and $\mathrm{Al}$ may play a crucial part in formation of these complex corrosion products, as they may possess synergistic effects [66]. Furthermore, under conditions of standardised salt spray (SST) testing, the formation of stable layered-double hydroxides (LDH) were attributed to the enhancements in corrosion resistance [67], [68]. The simple presence of $\mathrm{Mg}$ has been suggested to encourage the formation of LDH compounds, earlier than might be observed without $\mathrm{Mg}$ [66]. Contrarily, results obtained by Prosek et al. demonstrated that LDH were present on all but the $\mathrm{Zn}-\mathrm{Mg}$ alloy free of $\mathrm{Al}$, and this outperformed that of the ZMA alloys in this study [72]. Therefore, the mechanism with which LDH can provide corrosion protection in these alloys is not definitive. Considering the discussed research, it seems likely that Mg-based compounds are the primary factor for the improved corrosion performance observed within ZMA alloys. However, the oxide layers of both $\mathrm{Mg}$ and $\mathrm{Al}$ are prospective contributors as well. This body of work has studied the corrosion behaviour of ZMA under immersion conditions, whereas much of the previous work on this class of alloy has proceeded under different conditions. The high solubility of most magnesium-containing compounds may therefore only provide a short-term effect in terms of protection, and instead rely on zinc-based corrosion product formation in latter stages; simonkolleite would be one such stable compound, forming in preference to that of zincite [66]. The presence of Al would also support enhanced performance under chloride load due to the Al-rich oxide layer [67], surplus to the likely formation of LDH incorporating Al upon its dissolution, beyond the initial stages of corrosion [66].

\subsection{Conclusions}

The SVET was used to map the corrosion behaviour of three zinc-magnesium-aluminium alloys containing varying degrees of magnesium and aluminium. The data was used to distinguish mechanism and performance ranking amongst the alloys in an aqueous $1 \% \mathrm{w} / \mathrm{v} \mathrm{NaCl} \mathrm{pH} 7$ electrolyte. The SVET revealed that the extent of corrosion diminished for increasing amounts of $\mathrm{Mg}$ and $\mathrm{Al}$ present in the alloyed coatings, as given by the semi-quantitative metal loss values. The current density maps generated from SVET echoed the same trend that a higher alloy content coincided with improved corrosion resistance, alongside a reduced extent of anodic evolution with time and a fall in total number of anodic sites present.

The microstructure of each ZMA alloy was characterised using optical light microscopy, scanning electron microscopy (SEM), and energy dispersive X-ray spectroscopy (EDS). The images showed the microstructure to vary with the volume fraction of eutectic increasing in 
tandem with greater $\mathrm{Mg}$ and $\mathrm{Al}$ additions, and an associated drop in primary $\mathrm{Zn}$-rich phase. The morphological differences between each alloy exhibited a size reduction in primary $\mathrm{Zn}$ phase with larger alloying additions, most notably in ZMA 3. The markedly refined microstructure for ZMA 3 was likely consequent of a higher production cooling rate, than strictly an effect of increased $\mathrm{Mg}$ and $\mathrm{Al}$ presence. The eutectic phases were notable, consisting of a binary and ternary composition as revealed by SEM coupled with EDS.

Time-lapse optical microscopy was used effectively to monitor the microstructure of a corroding surface in situ. Corrosion was observed to initiate in the eutectic phases rich in magnesium, as confirmed by higher resolution SEM imaging which exhibited initial dissolution of $M g Z n_{2}$ phase. The preferential attack of eutectics was evident in each scenario, whereby the remaining zinc-rich phases were indeed corroded once the eutectic attack had taken place. The time-lapse data was subjected to image analysis by plotting the area of anodic attack for a series of given time-lapse intervals as a function of time. The overall results disclosed an identical trend to SVET in terms of performance ranking, showing that the higher alloyed samples delivered increased corrosion resistance levels via reduced kinetics.

The gathered results exhibited a distinct relationship of improved corrosion performance levels according to increases in magnesium and aluminium additions to a zincbased alloy. This favourable corrosion behaviour can be linked to the potentially increased formation of $\mathrm{Mg}$ and $\mathrm{Al}$ oxides (i.e. $\mathrm{MgO}, \mathrm{Al}_{2} \mathrm{O}_{3}$ ) on the surface; the semiconducting properties of these compounds would limit rates of electron transfer and thereby, reduce the extent of cathodic oxygen reduction occurring in a given period. The higher reactivity of magnesium encouraged the preferential attack of magnesium-rich phases, and the presence of both magnesium and aluminium and their associated corrosion products, such as $\mathrm{Mg}(\mathrm{OH})_{2}$ most likely hindered formation of less protective zincite $(\mathrm{ZnO})$. The underlying mechanism for this is understood to be due to the buffering effect of magnesium at higher $\mathrm{pH}$ ranges, favouring the precipitation of stable and more protective corrosion product species. These protective species were likely composed of magnesium- and zinc-based compounds during initial stages, followed by stable zinc-based compounds in the form of hydrozincite and simonkolleite, and aluminiumcontaining layered double hydroxides. 


\section{Chapter 4 The role of $\mathrm{pH}$ variation on the corrosion behaviour of a zinc-magnesium-aluminium alloy}

\subsection{Introduction}

A galvanising alloy may be used for a range of applications, externally or otherwise, and is likely to face exposure to a diverse set of environmental conditions. These circumstances often lead to changing $\mathrm{pH}$ conditions, which can dramatically impact the corrosion performance and thus, lifetime of the galvanised steel product. ZMA alloys are a more recent advent in galvanising alloy composition, and provide improved corrosion resistance for reduced coating weights comparative to traditionally employed counterparts [61]. The microstructures and corrosion behaviours of three zinc-magnesium-aluminium (ZMA) alloys under full aqueous conditions were studied in the previous chapter, albeit at neutral pH. Accordingly, this current body of work considers, in part, the effect that $\mathrm{pH}$ variation has on the corrosion behaviour of a selected ZMA alloy-coated steel. This is based on the likelihood of varied pH environments inservice.

This chapter focuses on a single ZMA alloy, specifically ZMA 2, due to the combination of balanced performance (as documented in the previous chapter) and its relevance from an industrial perspective; the alloy composition is detailed in 3.2.1. The effect of $\mathrm{pH}$ variation on ZMA was examined using scanning vibrating electrode technique (SVET) to resolve localised corrosion features and assess relative corrosion performance. The differing $\mathrm{pH}$ conditions were also studied on a microstructural level in situ, using time-lapse optical microscopy. These corrosion measurements were supported by open-circuit potential and potentiodynamic polarisation testing in conjunction with a potentiostat, to expand on any findings. Additionally, gravimetric analysis was performed to validate corrosion performance results.

\subsection{Experimental}

\subsubsection{Metallic specimens}

The details of the selected ZMA alloy composition for this chapter of work are detailed in Table 4.1. The given sample alloy was supplied by the French Corrosion Institute, in the asmanufactured state from a commercial producer. 
Table 4.1 Composition of the selected zinc-magnesium-aluminium alloy coating

\begin{tabular}{|c|c|c|c|c|}
\hline Sample & Zn (wt\%) & $\mathrm{Mg}(w t \%)$ & $\mathrm{Al}(w t \%)$ & $\begin{array}{c}\text { Coating thickness } \\
(\mu \mathrm{m})\end{array}$ \\
\hline ZMA 2 & 96 & 2 & 2 & 10 \\
\hline
\end{tabular}

\subsubsection{Sample preparation}

As outlined in 2.1.2.1, sample alloy sheets were guillotined into $20 \mathrm{~mm} \times 20 \mathrm{~mm}$ coupons, followed by mounting, polishing to a $1 \mu \mathrm{m}$ surface finish, and a cleaning procedure using distilled water and ethanol. A Nital etchant solution (2\%) was used to reveal the microstructure, followed by the prior cleaning procedure. To perform SVET experimentation, adhesive PTFE tape was used to mask the sample surface, excluding an area of $c a .10 \mathrm{~mm} \times 10 \mathrm{~mm}$ exposed for study. A largely similar masking procedure was followed for time-lapse microscopy experimentation, whereby a circular feature of approximately $0.785 \mathrm{~mm}^{2}$ area ( $1 \mathrm{~mm}$ diameter) was instead exposed. Further electrochemical and polarisation testing involved preparations described in 2.5-2.6; samples removed from their mounts prior to masking were subsequently fitted securely in the custom-made sample holder, revealing an area of $0.95 \mathrm{~mm}^{2}$ exposed.

\subsubsection{Experimental methods}

SVET was in part used to ascertain the differences in corrosion performance and localised mechanisms, performing scans according to $\mathrm{pH}$ variation. This data allowed the production of spatially-resolved maps, visually representing surface corrosion events, as well as data manipulation leading to a value of semi-quantitative metal loss and area-averaged anodic current density plots. SVET experimentation was performed using samples of ZMA 2 under immersion conditions, specifically in a baseline $1 \% \mathrm{w} / \mathrm{v} \mathrm{NaCl}$ electrolyte adjusted to $\mathrm{pH} 7$. Samples freely corroded over the 24-hour experimental time-frame. All samples were scanned immediately upon experimental onset, and hourly therein until the close of experiment. The SVET apparatus, operation, and calibration using the two-compartment cell is described in detail in 2.2. To maintain the accuracy of results, a minimum of two repeat scans were performed for each parameter varied.

To enable the study of $\mathrm{pH}$ variation on $\mathrm{ZMA}$, electrolytes were adjusted to $\mathrm{pH} 3,7$, and 10. These adjustments to $\mathrm{pH}$ involved incremental additions of dilute $\mathrm{HCl}$ or $\mathrm{NaOH}$. In addition to SVET, time-lapse optical microscopy was used to study the experimental parameters within this body of work. This version of the technique enables the study of corroding samples under immersion conditions in situ, and at a microstructural level. Following relevant preparation, samples were fitted to the glass tank, subsequently immersed in the $1 \% \mathrm{NaCl}$ electrolyte, and 
the microscope lens positioned for image capture. Digital images were captured successively in 2-minute intervals over the course of the experiment. The operation of the equipment is further outlined in 2.3. Electrochemical tests, in the form of open-circuit potential (OCP) and potentiodynamic polarisation curves were all conducted versus a saturated calomel electrode (SCE). For the means of polarisation curves, the tests ranged between approximately $-1.5 \mathrm{~V}$ and $-0.6 \mathrm{~V} v s \mathrm{SCE}$; these polarisation tests were conducted at a sweep rate of $1 \mathrm{mV} \mathrm{s}^{-1}$. This form of electrochemical testing is further detailed in 2.5-2.6. To verify the accuracy of SVET-measured results, gravimetric analysis was performed for the three variables of electrolyte $\mathrm{pH}$ condition. This was performed as 24-hour tests to adequately mimic the SVET time-frame and increase the comparability of the testing procedures, the details of which can be found in 2.7.

\subsection{Results and discussion}

The results relating to the $\mathrm{pH} 7$ condition for ZMA 2, as discussed in Chapter 3, have been included to illustrate the differences between the varying $\mathrm{pH}$ conditions for the alloy in question.

\subsubsection{Assessment of $\mathrm{pH}$ variation on the corrosion behaviour of a ZMA alloy using SVET} The SVET has been utilised to monitor the surface corrosion activity for a ZMA 2 alloy in $1 \% \mathrm{w} / \mathrm{v}$ electrolyte, at conditions ranging from $\mathrm{pH} 3-10$. The results generated from SVET encompass spatially-resolved current density maps, semi-quantitative values of metal loss, and areaaveraged anodic-going current density plots. The associated procedures to obtain these data sets are outlined in 2.2. As discussed in Chapter 3, the predominant element in the sample alloy is zinc and as such, was selected as the element involved in the dissolution during any metal loss calculations. Figure 4.1 represents the values of metal loss derived from SVET data according to the change in electrolyte $\mathrm{pH}$ conditions. The results displayed that at more extreme $\mathrm{pH}$ conditions away from approximately neutral $(\mathrm{pH} 7)$, the extent of corrosion was reduced. At $\mathrm{pH} 10$, the values were found to be less than $21 \%$ to that of $\mathrm{pH} 7$, the lowest recorded and thus best performing. At pH 3, the reduction in metal loss was closely followed, offering a reduction in excess of $70 \%$ comparative to $\mathrm{pH} 7$. However, $\mathrm{pH} 3$ did offer the largest extent of error between experiments, whereas this was minimal for the remaining parameters. The results indicated that at $\mathrm{pH} \mathrm{7,} \mathrm{the} \mathrm{ZMA} \mathrm{alloy} \mathrm{offered} \mathrm{the} \mathrm{least} \mathrm{corrosion} \mathrm{resistance} \mathrm{levels,}$ with the highest $\mathrm{pH}$ condition providing the greatest levels of protection. 


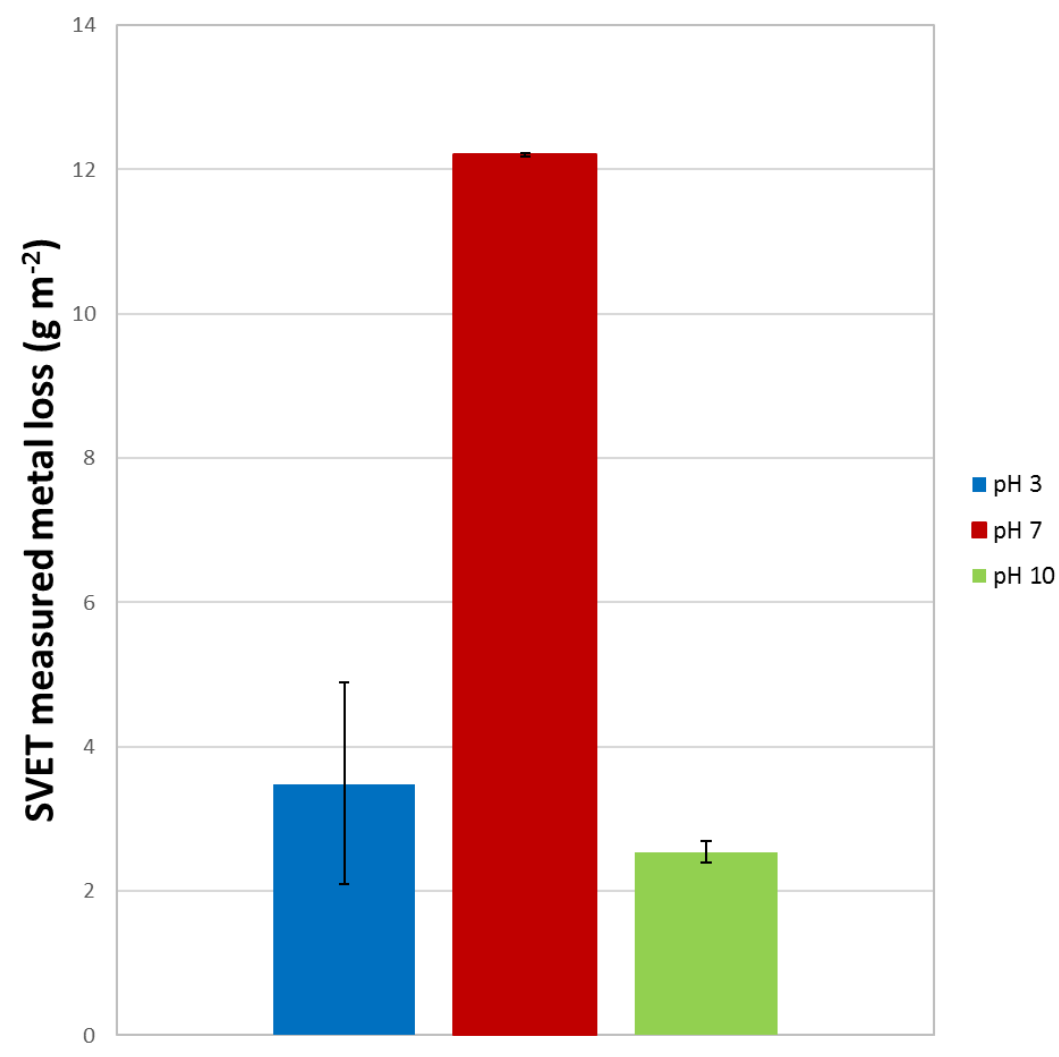

Figure 4.1 SVET estimated metal loss over 24-hour experiment time for ZMA 2 in $1 \% \mathrm{NaCl}$ at varying $\mathrm{pH}$

The corrosion activity has been visually represented in the form of current density maps generated for each full scan. A selection of scans, given in Figure 4.2 A-C, indicate the changes in localised corrosion activity realised by SVET. Considering Figure $4.2 \mathrm{~A}$ and the hour 1 scan for $\mathrm{pH} 3$, it was immediate that anodic activity was present over a large area of the exposed surface. The majority of these were present on the upper half of the sample and existed as anodic island-like features. There were also smaller anodic-going features akin to point anode profile, but of a lower intensity level. At hour 12 , the overall intensity was observably diminished. The weaker anodic activity however encompassed the majority of the active surface in a near-continuous fashion. The final scan at hour 24 further evidenced a decreasing magnitude of activity, alongside a reduced coverage of anodic sites. The anodic activity retained the island-like profile observed from previous hours, as well as its continuity. As discussed in Chapter 3, the scan at hour 1 for $\mathrm{pH} 7$ (Figure 4.2 B) displayed multiple distinctive point anodes of high intensity on the surface. At hour 12, these point anodes were observed to progress outwards in a radial fashion to form 'ring' anodes, accompanied by the formation of a fresh anodic site. Generally, these ring anodes were of a lower intensity than the point anodes present in hour 1. By hour 24 many of the original anodic features were no longer active, although remnants of their existence were still present from the scan. A larger island of anodic 
activity formed on the surface, varying in intensity depending on the position of the site. Whilst the anodic features were more persistent across the experiment time-frame relative to the $\mathrm{pH}$ 3 experiment, the intensity was somewhat greater. The prominent variation between both the $\mathrm{pH} 3$ and $\mathrm{pH} 7$ experiments indicated a change in corrosion mechanism, as given by the variation in surface activity. Figure $4.2 \mathrm{C}$ displayed the presence of multiple point anodes on the surface at hour 1, the intensity of which varied depending on the individual anodic site. By hour 12 these sites persisted but were also seen to diminish in intensity on the whole. The final scan of hour 24 revealed the continued lifetime of the original anodic features, yet of a lower intensity than observed at the beginning of the experiment. A ring anode formation was also present in some of these sites, the evolution of which was much more gradual comparative to the $\mathrm{pH} 7$ counterpart at hour 12 . This dampened anodic evolution can be understood by the reduced levels of metal loss recorded by the SVET for $\mathrm{pH} 10$. The action of the corrosion events had a greater degree of similarity between $\mathrm{pH} 7$ and $\mathrm{pH} 10$ experiments, which exhibited multiple point anodes and a ring anode profile evolution; the differences between these existed in the extent of the anodic evolution, corroborated by Figure 4.1. This trend is echoed in the anode analysis plotted in Figure 4.3, with pH 7 displaying a degree of similarity in rate of anodic growth to $\mathrm{pH} 10$, but of greater magnitude. The $\mathrm{pH} 3$ condition clarified the larger anodic front that continued to grow until the latter stages of the experiment, whereby the size of the anodic feature diminished. The anodic area analysed for the $\mathrm{pH} 3$ condition was not of the more regular point anode profile, and instead was island-like in shape. 
A
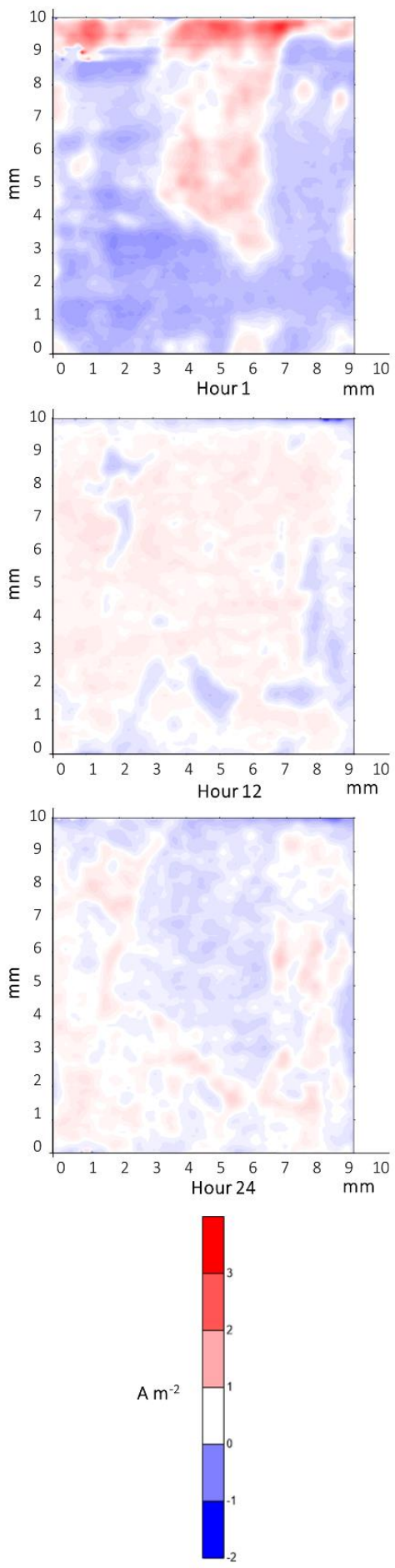

B
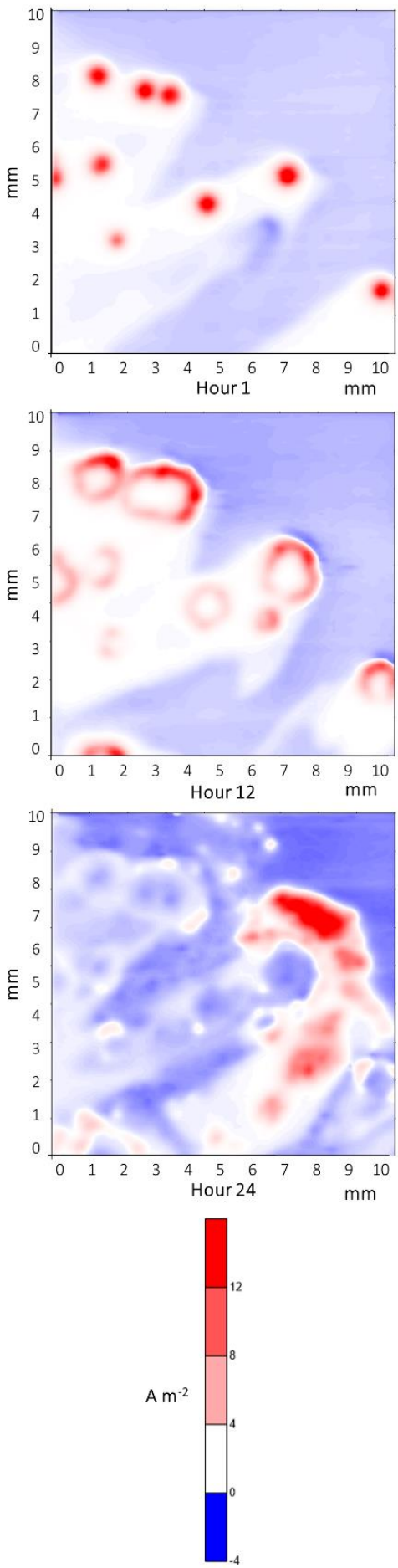

C
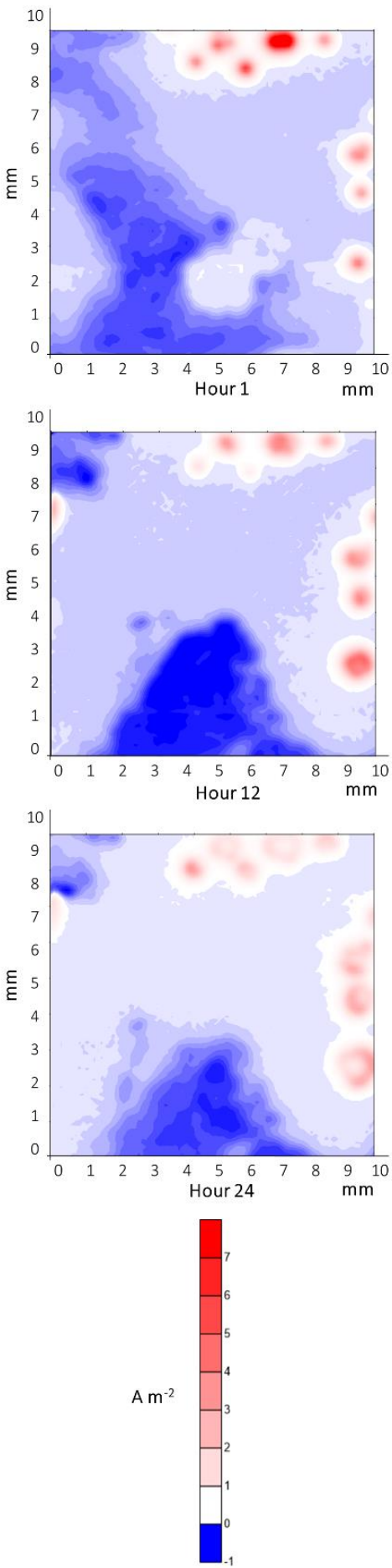

Figure 4.2 SVET-measured current density maps of ZMA 2 in $1 \% \mathrm{NaCl}$ electrolyte at A) pH 3, B) pH 7, and C) $\mathrm{pH} 10$ 


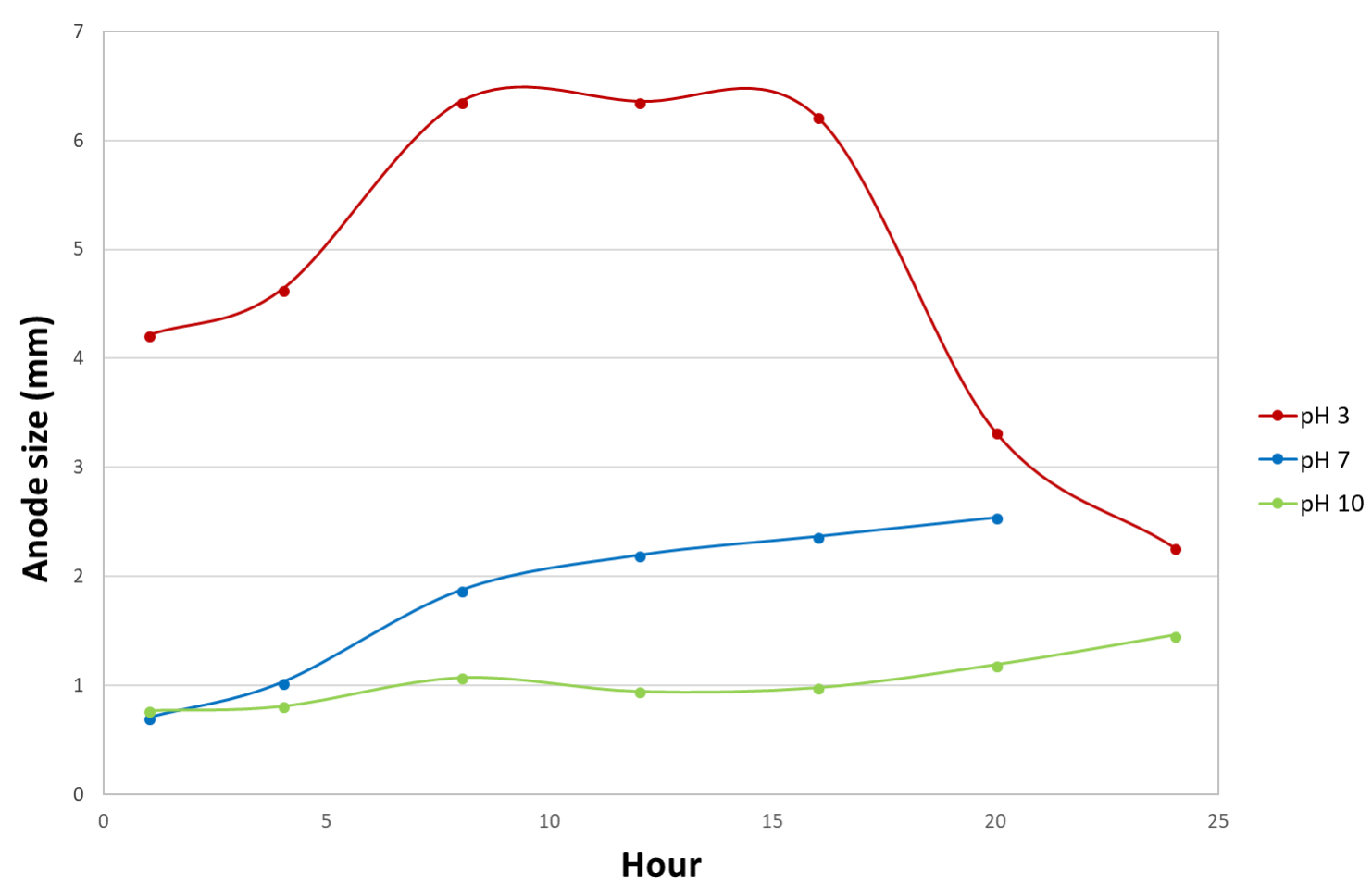

Figure 4.3 Plot of SVET anode size as a function of time for the three $\mathrm{pH}$ conditions

The area-averaged anodic current density $\left(\mathrm{Ja}_{\mathrm{t}}\right)$ plotted as a function of time is given in Figure 4.4, for the three different $\mathrm{pH}$ conditions considered. The plot demonstrated a steep rise in Jat at the initial scan for all $\mathrm{pH}$ conditions. Whilst at $\mathrm{pH} 7$ the curve was observed to increase further at a less steep gradient, the remaining $\mathrm{pH}$ conditions were observed to fall at hour 1 . In the case of $\mathrm{pH} 3$, the curve gradually diminished until approximately hour 10 , whereby the associated anodic activity varied for several hours, stabilising from hour 18. Despite the similarity between $\mathrm{pH} 3$ and $\mathrm{pH} 7$ within the first hour, the $\mathrm{pH} 3$ anodic-going activity notably fell beyond this point comparative to $\mathrm{pH}$ 7. For $\mathrm{pH} 10$, the curve illustrated a sharp fall in anodic activity between hours 1-4, where a slight increase was visible; from this point the plot was evidently more stable and displayed a gradual fall in the anodic-going values from hour 8 and beyond. The $\mathrm{pH} 10$ curve upheld the greatest extent of stability throughout whilst offering the lowest $\mathrm{Ja}_{\mathrm{t}}$ values overall. As the data associated with Figure 4.4 was derived from the SVET data, it understandably parallels the observations that $\mathrm{pH} 10$ offered the most favourable corrosion resistance levels, and $\mathrm{pH} 7$, the least. The results thus far indicate that the ZMA 2 alloy provided a greater degree of protection from corrosion in the more extreme conditions of $\mathrm{pH}$, than that closer to neutral. 


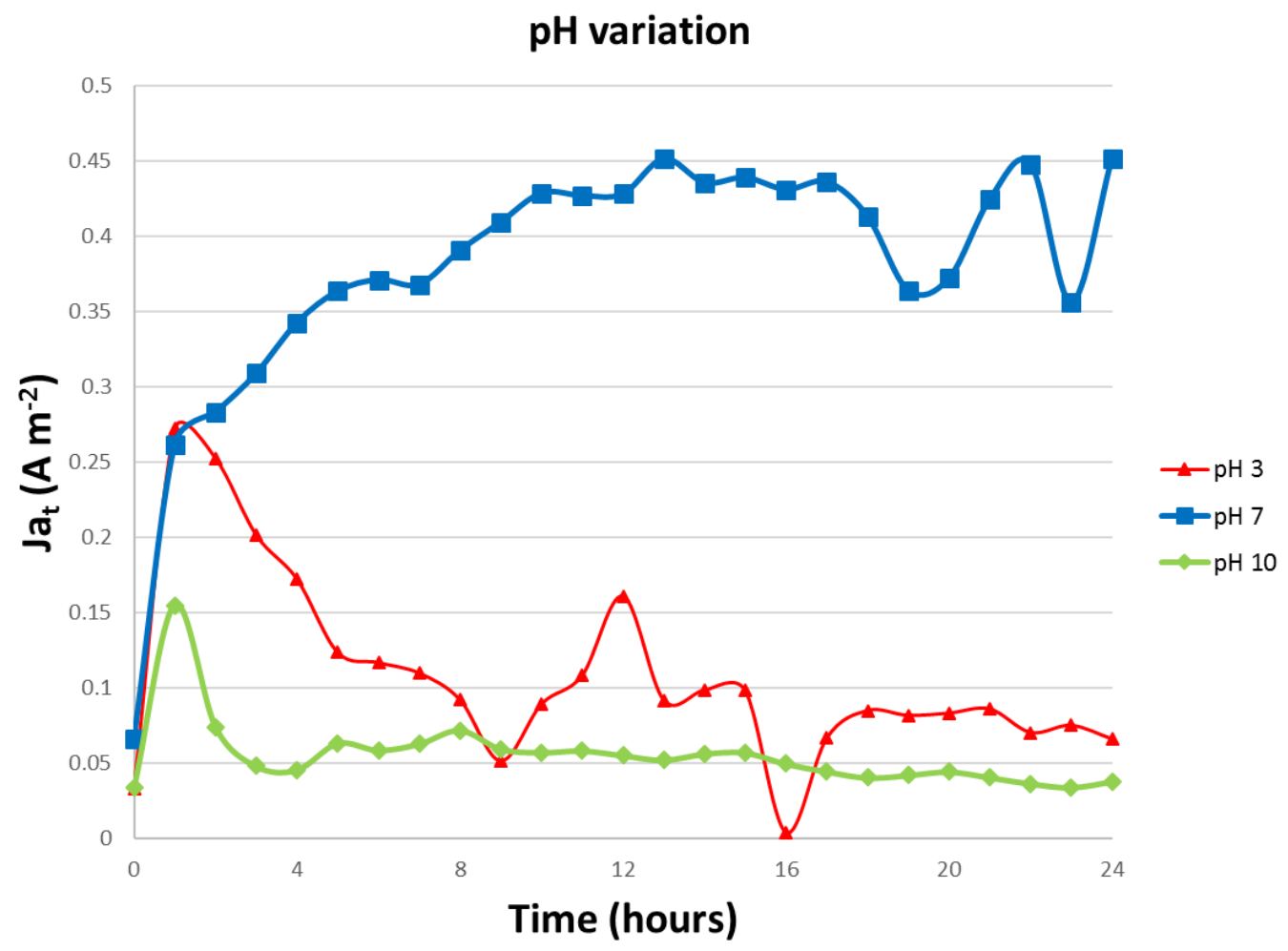

Figure 4.4 The area-averaged anodic-going current density $\left(\mathrm{Ja}_{\mathrm{t}}\right)$ plotted as a function of time for ZMA 2 alloy for the studied $\mathrm{pH}$ range in $1 \% \mathrm{NaCl}$ electrolyte

\subsubsection{Monitoring of ZMA corrosion activity using time-lapse optical microscopy}

The ZMA samples were observed on a microstructural level according to the three $\mathrm{pH}$ conditions, as given in Figure 4.5. Whilst images were captured at two-minute intervals from the onset of the experiment, Figure 4.5 includes images captured at 9-hour intervals. At pH 3 (Figure $4.5 \mathrm{~A}-\mathrm{D}$ ), following 9 hours immersion corresponding to image $\mathrm{B}$, a selection of the surface was noticeably darkened. This darkened appearance was apparent for both eutectic and primary dendrites phases, although there were also regions of primary dendrite that were distinctly unaffected by this darkened appearance. Continuation of the experiment into images C-D demonstrated an increased darkening, whilst leaving a large proportion of the unaffected area in the same state. It can be understood that the darkening observed on select areas of the surface was that of anodic attack, whilst the intact regions of primary dendrites likely adopted cathodic activity. The observed attack by the end of the experiment was somewhat extensive.

As discussed in Chapter 3, the pH 7 experiment of Figure 4.5 E-H displayed the formation of focal anodic activity within the eutectic phases, surrounded by a ring of corrosion product at a given distance from said activity. This provided evidence of the transition from anodic to cathodic activity outside the boundary of the corrosion product ring. Progression of the experiment highlighted the advancing front of anodic activity, accompanied by the 
development of this corrosion product ring. This $\mathrm{pH}$ condition exhibited a localised form of corrosion overall, which was observed to be the case for the pH 10 experiment also. Figure 4.5 I-L displayed distinct anodic features accompanied by a ring of corrosion product. As shown in image $\mathrm{J}$, this ring of corrosion product was notably more proximal to the regions of anodic attack comparative to the $\mathrm{pH} 7$ counterpart. Additionally, the extent of anodic attack was less pronounced for the case of $\mathrm{pH} 10$. Both the anodic activity and development of the corrosion product ring were observed to progress into images $\mathrm{K}-\mathrm{L}$, but in a less marked fashion than for $\mathrm{pH}$ 7. The final interval of image $L$ revealed the corrosion product to extend across a large proportion of the surface, with minimal growth of the anodic sites between image intervals.

In the case of $\mathrm{pH} 7$ and $\mathrm{pH} 10$, anodic attack occurred in a localised manner, and a ring of corrosion product was observed to precipitate in accordance with this attack. However, the $\mathrm{pH} 3$ experiment ensued in a different manner; the corrosive attack appeared to proceed in a more generalised fashion. The anodic attack occurred over larger regions of the surface, in the eutectic and primary zinc-rich phases, with other intact primary dendrites seemingly acting in a cathodic manner. This suggests that the behaviour of a ZMA alloy in different in acidic chloride media, whereby a shift in corrosion mechanism is apparent. This was not the case for both the neutral and alkaline conditions, which maintained a localised form of corrosion during timelapse microscopy studies. 
$\mathrm{pH} 3$
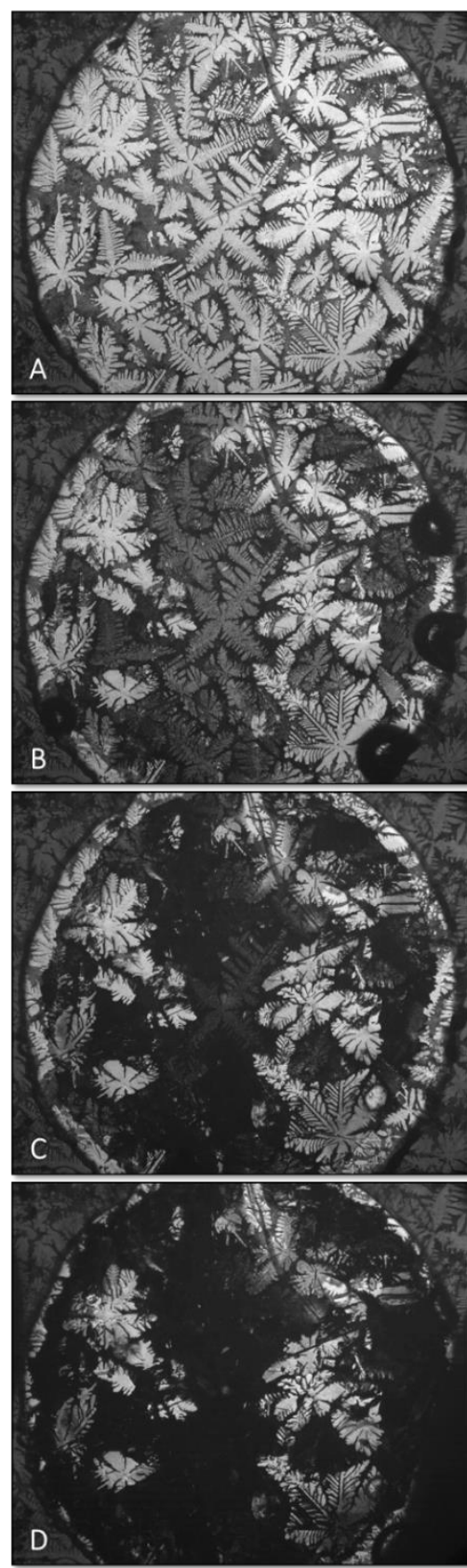

$\mathrm{pH} 7$
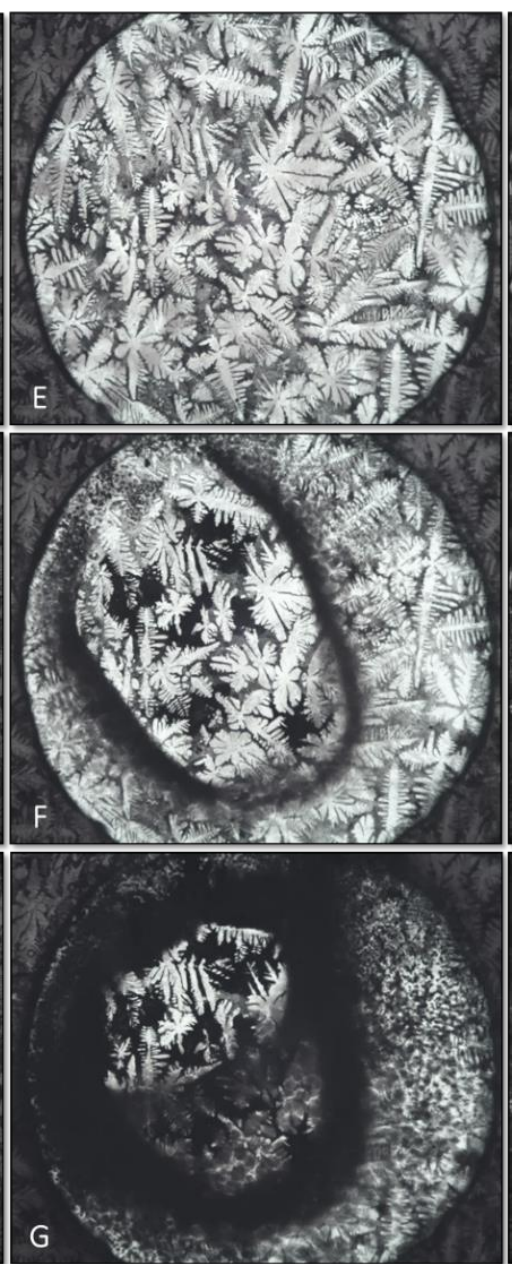

pH 10
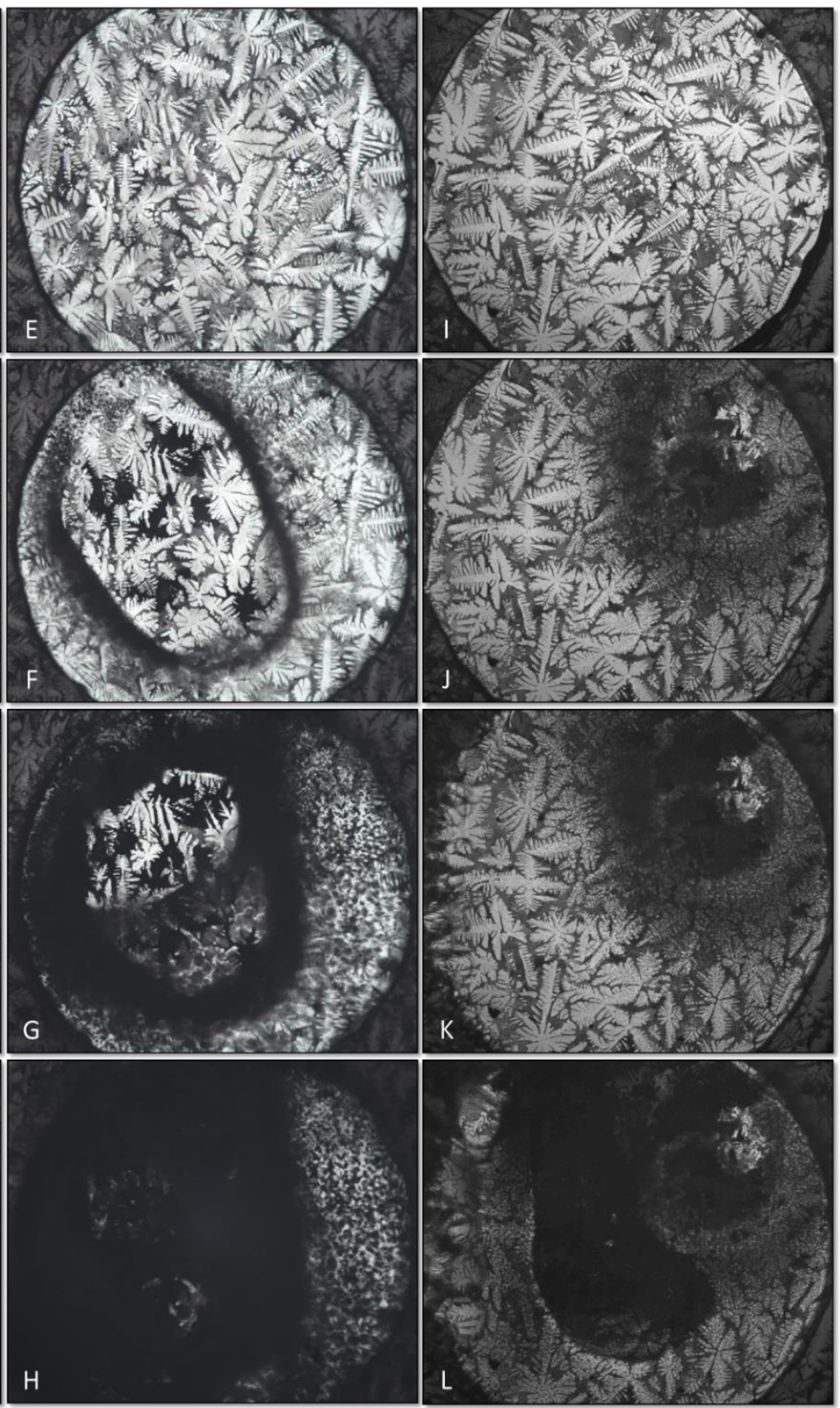

$250 \mu \mathrm{m}$

Figure 4.5 Time-lapse microscopy images taken at intervals of 9 hours in a $1 \% \mathrm{NaCl}$ electrolyte for (A-D) pH 3, (E-H) pH 7, and (I-L) pH 10

\subsubsection{Electrochemical study of $\mathrm{pH}$ variation on ZMA 2 alloy}

A series of electrochemical experiments were performed to further study the effect of varying $\mathrm{pH}$ solution conditions on the behaviour of a ZMA 2 alloy. In conjunction with a potentiostat, both open-circuit potential and potentiodynamic polarisation work was completed. Opencircuit potential testing involved immersing samples in their designated solution to freely corrode for approximately 80 minutes, to reach a stable rest potential value. The polarisation testing was conducted under de-aerated conditions and involved allowing each sample to reach 
a stable value, freely corroding at its open-circuit for $c a$. 80 minutes, before conducting the polarisation sweep. These were conducted between the approximate range of $-1.60 \mathrm{~V} v$ s SCE up to $-0.60 \mathrm{~V}$ vs SCE, beginning from the most cathodic potential.

The results given in Figure 4.6 demonstrated that all samples showed a degree of stabilisation with time. The $\mathrm{pH} 3$ sample exhibited the lowest value of potential (vs SCE), with $E_{\text {corr }}$ values increasing according to higher values of $\mathrm{pH}$. As stipulated by the galvanic series (see Figure 1.3), a decrease in free corrosion potential $\left(E_{\text {corr }}\right)$ corresponds to a heightened thermodynamic propensity for corrosion; accordingly, the anodic shift visible from the $\mathrm{pH} 10$ sample should grant the greatest extent of nobility, with the $\mathrm{pH} 3$ condition contrarily being the most likely to corrode. However, whilst the thermodynamic measurement provides an indicator of the likelihood of corrosion, this does not necessarily translate to corrosion performance or ranking, as kinetic information is not portrayed from this type of measurement [94]. These results were discordant with that obtained via SVET, which suggested that $\mathrm{pH} 3$ yielded better performance than that of $\mathrm{pH} 7$ conditions. Yet, the time-lapse microscopy data showed a greater extent of corrosive attack for the $\mathrm{pH} 3$ condition than its higher $\mathrm{pH}$ counterparts, in agreement with the indications associated with open-circuit potential data.

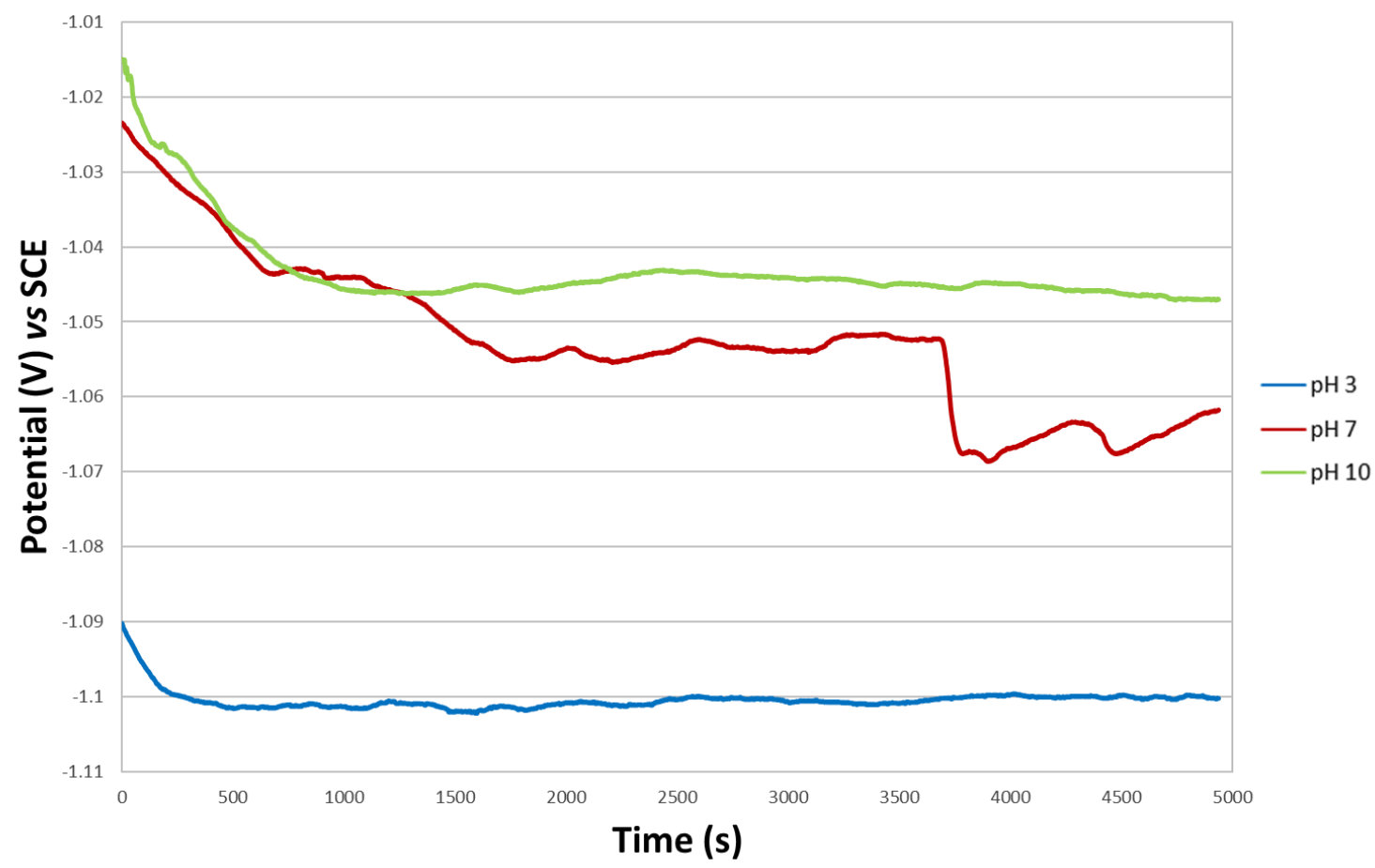

Figure 4.6 Plot of open-circuit potential testing (versus SCE) for the varied $\mathrm{pH}$ condition in $1 \%$ $\mathrm{NaCl}$ electrolyte

The potentiodynamic polarisation data completed under de-aerated conditions is plotted in Figure 4.7. The $\mathrm{E}_{\text {corr }}$ values did not fully align with those obtained in Figure 4.6, revealing the pH 
7 and $\mathrm{pH} 10$ experiments to adopt more negative potentials. For $\mathrm{pH} 7$, the anodic branch of the curve displayed a region of passivity for over-potentials of approximately $70 \mathrm{mV}$. The $\mathrm{pH} 10$ counterpart also demonstrated this, showing an extended state of passivity for over-potentials of $140 \mathrm{mV}$ between $-1.20 \mathrm{~V}$ to $-1.06 \mathrm{~V}$ vs SCE. Comparative to the $\mathrm{pH} 3$ counterpart, these higher $\mathrm{pH}$ conditions showed lower values of $\log (\mathrm{I})$, with $\mathrm{pH} 10$ adopting the lowest values between the three at $-1 \mathrm{~V}$ vs SCE. The $\mathrm{pH} 3$ curve did not exhibit regions of passivity at anodic potentials and generally suggested a more pronounced extent of corrosion corresponding to increased corrosion currents. This conflicts in part with the data gathered by SVET, but aligns more with the time-lapse microscopy data of Figure 4.5 which indicated less favourable corrosion resistance levels for that of $\mathrm{pH} 3$ conditions. However, the $\mathrm{pH} 10$ condition unanimously offered favourable results within the data sets.

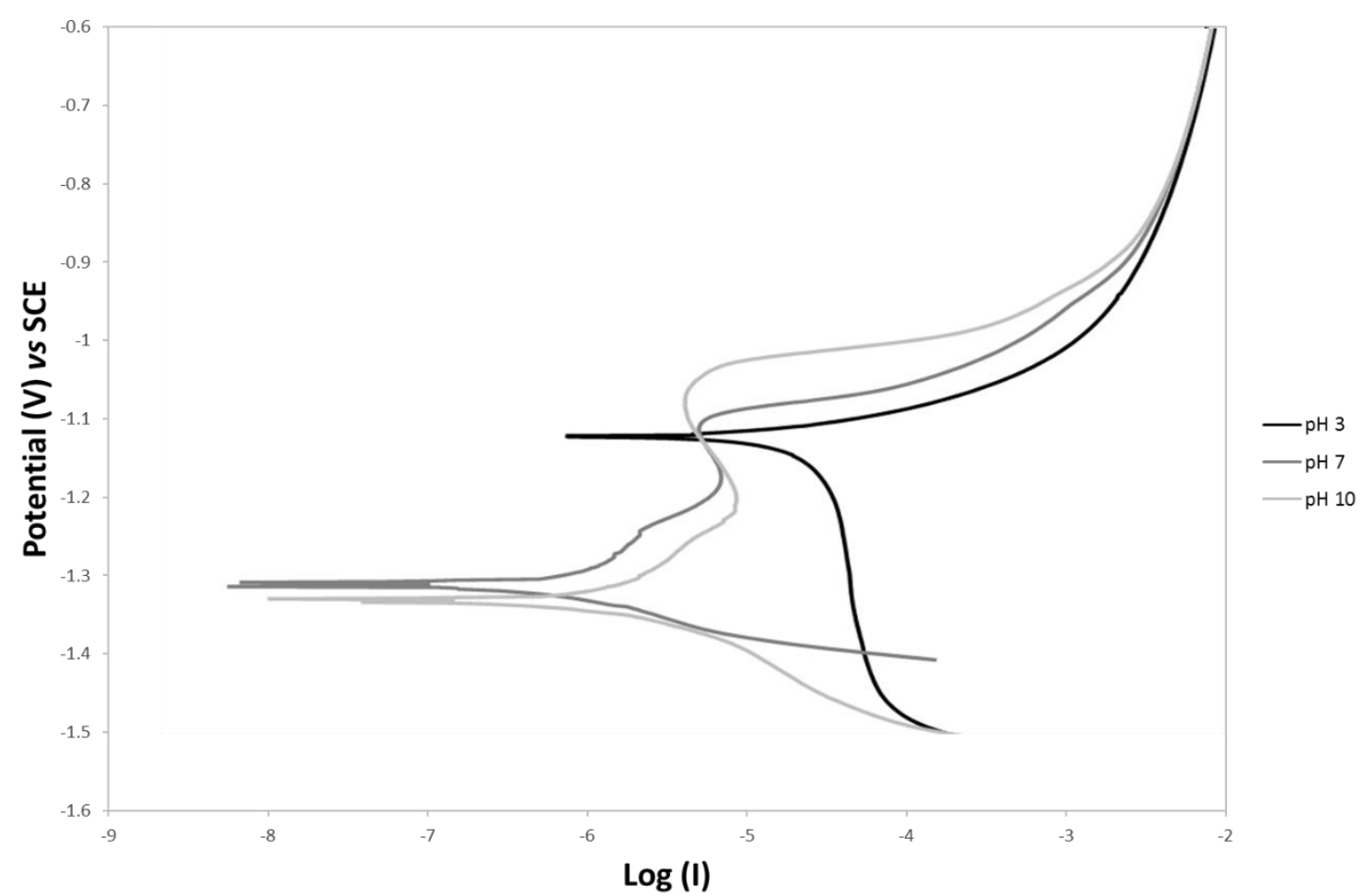

Figure 4.7 Plot of polarisation curves according to $\mathrm{pH}$ variation in a de-aerated $1 \% \mathrm{NaCl}$ electrolyte

\subsubsection{Confirmation of SVET-realised metal loss using gravimetric analysis}

SVET can provide useful data to elucidate corrosion behaviour, including the metal loss values that can be extracted. However, the semi-quantitative nature of such metal loss values may lead to misinterpretation of data. As such, gravimetric analysis was used to support the obtained results. This supporting experiment was performed as set out in ISO 8407, and is fully described in 2.7. For comparability, samples were immersed in solution according to $\mathrm{pH}$ condition for 24 hours so as to replicate that used for SVET experimentation. 


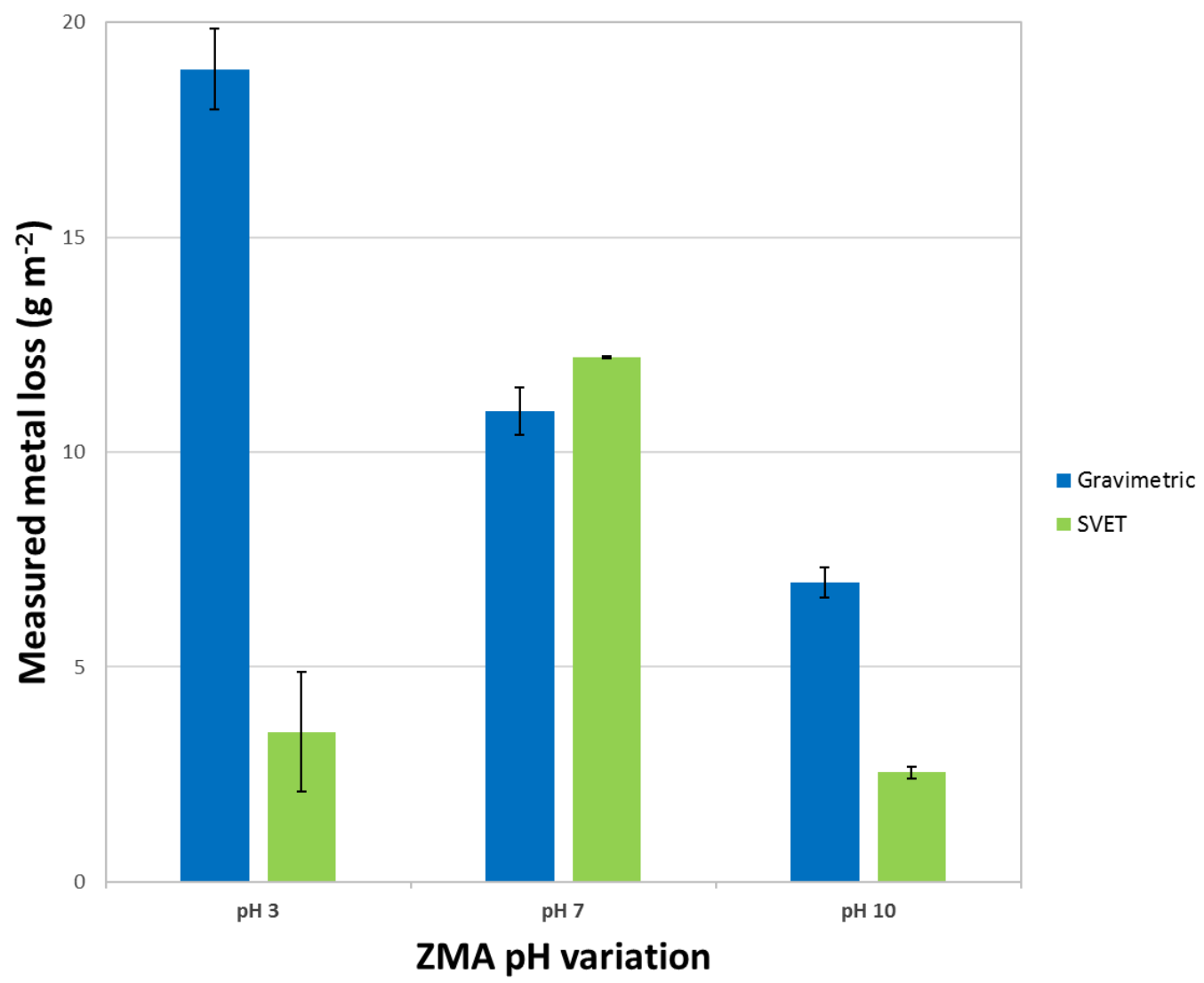

Figure 4.8 Chart of measured metal loss from gravimetric and SVET methods including the studied $\mathrm{pH}$ range in a $1 \% \mathrm{NaCl}$ electrolyte

As plotted in Figure 4.8, the comparison of SVET and gravimetric data for metal loss is given. The $\mathrm{pH} 7$ condition exhibited good agreement, revealing the SVET to have slightly overestimated the extent of corrosion. At $\mathrm{pH} 10$, the data instead suggested an underestimation by SVET, with gravimetric values indicating a more than two-fold increase comparatively. Interestingly, the $\mathrm{pH} 3$ condition revealed a significant disparity, whereby gravimetric measurements realised a much greater metal loss than seen by SVET. The overall trend also strongly suggests that corrosion resistance levels increased according to $\mathrm{pH}$ condition, with $\mathrm{pH} 10$ demonstrating the lowest measured metal loss values.

There are limitations associated with both techniques, which may lead to some discrepancies like that seen in Figure 4.8. The SVET is restricted in that it does not detect the direct surface current density on a corroding sample and is limited in resolution. It also assumes that corrosion is consistent between each SVET scan. However, corrosion is a dynamic process and a consistent corrosion rate may not transpire. This may in part explain the underestimation associated with SVET measurements observed. On the other hand, gravimetric analysis is a post-corrosion, bulk measurement technique that uses an analytical balance; the highly sensitive values associated with this analysis may be subject to reasonable degrees of error. 
This is well suited to the SVET that detects ionic current flux under immersion conditions. Conversion of the data to $\mathrm{g} \mathrm{m}^{-2}$ would also amplify any error corresponding to the measurements. Furthermore, the sample area exposed during gravimetric experiments was larger than that for SVET, potentially affecting the results. This may account for inconsistencies in the gravimetric results. Such limitations may explain the observed differences for both $\mathrm{pH} 7$ and $\mathrm{pH}$ 10. Conversely, the sheer extent of the conflicting result for $\mathrm{pH} 3$ suggested that other factors were responsible. The time-lapse microscopy data of Figure 4.5 revealed a shift in corrosion mechanism at $\mathrm{pH} 3$, demonstrating a more generalised form of attack on the surface. The extent of this attack was also much more prominent than that suggested by SVET. Both open-circuit potential and polarisation data sets agreed with the time-lapse microscopy data, showing $\mathrm{pH} 3$ to possess the least favourable corrosion properties. Ultimately, this strongly indicated that the SVET results corresponding to the $\mathrm{pH} 3$ condition were not as representative, and that the more acidic conditions were in fact detrimental to the ZMA corrosion performance.

\subsubsection{Exploration of the effect of $\mathrm{pH}$ variation on corrosion behaviour of ZMA 2}

As outlined by the Pourbaix diagrams for $\mathrm{Zn}, \mathrm{Mg}$, and $\mathrm{Al}$, in Figure 1.5-1.7, at $\mathrm{pH} 3$ conditions each element is understood to readily corrode, according to Equations 4.1-4.3, respectively. Whilst cathodic oxygen reduction is understood to occur at middle to higher $\mathrm{pH}$ environments, at the lower $\mathrm{pH}$ range, hydrogen evolution is prevalent (Equation 4.4). These correspond to a reduced formation of the poorly soluble corrosion products in the form of zinc hydroxide, magnesium hydroxide, and alumina, all of which can impart reduced rates of corrosion. As these favourable corrosion products are less prevalent in the given acidic conditions, it can be realised that corrosion performance would diminish as a result.

$$
\begin{gathered}
Z n \rightarrow Z n^{2+}+2 e^{-} \\
M g \rightarrow M g^{2+}+2 e^{-} \\
A l \rightarrow A l^{3+}+3 e^{-}
\end{gathered}
$$




$$
\begin{gathered}
2 \mathrm{H}^{+}+2 e^{-} \rightarrow \mathrm{H}_{2} \\
2 \mathrm{H}_{2} \mathrm{O}+\mathrm{O}_{2}+4 e^{-} \rightarrow 4 \mathrm{OH}^{-} \\
\mathrm{Zn}^{2+}+2 \mathrm{OH}^{-} \rightarrow \mathrm{Zn}(\mathrm{OH})_{2} \\
\mathrm{Mg}^{2+}+2 \mathrm{OH}^{-} \rightarrow \mathrm{Mg}(\mathrm{OH})_{2}
\end{gathered}
$$

The Pourbaix diagram also defines that at higher $\mathrm{pH}$ conditions, up to a point, zinc hydroxide, magnesium hydroxide, and alumina are formed more readily, extending passivity to a sample. This coincides with cathodic oxygen reduction (Equation 4.5) in the $\mathrm{pH} 7$ and 10 range, potentially resulting in the formation of beneficial corrosion product species given in Equations 4.6-4.7. However, this alone is not a comprehensive representation of the corrosion products apparent on a ZMA surface, and a range of compounds have been observed experimentally within literature [47], [66], [70]-[72], [90], [91], [95]. The presence of chloride in the electrolyte will also affect the indications drawn from the corresponding Pourbaix diagrams, altering the associated corrosion product formation possible. It can be realised from this that the corrosion reactions occurring on the metal alloy surface will not be as straightforward using Pourbaix diagrams alone. Surplus to this, it cannot be assumed that the corrosion behaviour will coincide according to each alloy individually; the microstructure of the ZMA 2 alloy constitutes multiple phases, all of which contain varying proportions of each metal that constitute the ternary composition. When considering the $\mathrm{pH}$ environments studied, it may well be the case that the local $\mathrm{pH}$ is more extreme; for example, in the case of metallic cation build-up proximal to an anodic site, the $\mathrm{pH}$ may decrease more so than the bulk electrolyte $\mathrm{pH}$. Furthermore, the presence of a surface film such as $\mathrm{Mg}(\mathrm{OH})_{2}$ and its known buffering effect [72] could alter the state of the remaining alloys according to the associated Pourbaix diagrams, again leading to misinterpretation.

The time-lapse microscopy intervals of Figure 4.5 illustrated a shift in corrosion mechanism relative to the $\mathrm{pH} 7$ and 10 counterpart conditions. At these higher $\mathrm{pH}$ conditions, the anodic attack was observed to proceed in the eutectic phases, and only progressing to the more noble phases (zinc-rich dendrites) once anodic attack reached an appropriate size. At the 
$\mathrm{pH} 3$ environment, the shift to a more generalised form of corrosion demonstrated that the zinc-rich dendrites were, in most cases, the more noble phase of the ZMA 2 microstructure. Whilst the initiation of corrosion was still in the eutectic phases, the progression of this more generalised corrosion was less discriminate, proceeding to attack the primary dendrites in some, but not all, cases. The collection of results obtained via SVET may have led to the misinterpretation of the corrosion behaviour associated with pH 3 solution conditions, and so the limiting resolution of the SVET must be considered. As this scanning technique can realise localised corrosion within a given spatial resolution, a modification to generalised corrosion activity can be responsible for an underestimation in activity. If a corrosion cell is separated by a distance less than that of the SVET scan height, then this event will not be registered. This is because the iso-potential lines exist beneath the intersection point of the oscillating SVET probe, and therefore, will not be registered during a scan. Consequently, the prevalence of generalised activity will result in an underestimation of the corrosion events on the surface. This scenario may explain the differences observed between the forms of metal loss measurement at $\mathrm{pH}$ 3. To further this argument, the SVET current density maps of Figure 4.2 demonstrated a different mechanism for the lower $\mathrm{pH}$ condition. The formation of point anodes and their evolution to ring anodes, as seen for $\mathrm{pH} 7$ and $\mathrm{pH} 10$, was not visible; instead, anodic activity dominated large proportions of the surface, as was observed in Figure 4.3. Reinforced by the electrochemical data and gravimetric analysis, the results for the $\mathrm{pH} 3$ condition by SVET have been considered less representative than for the remaining $\mathrm{pH}$ solutions.

The increased corrosion resistance afforded by the higher $\mathrm{pH}$ counterparts can be considered via the formation of beneficial corrosion products. In ZMA alloys, the preferential dissolution of magnesium and its corresponding corrosion products are understood to be advantageous; $\mathrm{MgZn}_{2}$ phase is attacked in the first instance, and a Mg-rich oxide layer has been shown as the native oxide layer for this phase [90]. The author specified that this oxide layer possesses a large bandgap ( $c a .8 \mathrm{eV}$ ) relative to $\mathrm{ZnO}$. This larger bandgap is understood to impart a decreased ability for electron transfer, thereby reducing the rate of the cathodic reaction and providing improvements to the performance [71], [91]. In the case of $\mathrm{pH} 10$, the Pourbaix diagram for $\mathrm{Mg}$ (Figure 1.6) suggests that it would remain in a state of corrosion, having not reached the threshold to permit $\mathrm{Mg}(\mathrm{OH})_{2}$ formation. However, the $\mathrm{pH}$ conditions in the locale of the cathodic site can be understood as greater than that typically observed in bulk solution. This also may have been affected by the fact that the electrolyte in question was a chloride-containing solution, whereas the Pourbaix diagrams referred to are for water. Consequently, this local rise in $\mathrm{pH}$ may in fact have given rise to the development of magnesium 
hydroxide. Accordingly, the rapid formation of $\mathrm{Mg}(\mathrm{OH})_{2}$ and other $\mathrm{Mg}$-containing corrosion products have been proposed to hinder the development of less favourable zinc compounds [95]; this is attributed to a pH buffering effect by the Mg salts [47], [65], [72]. Whilst at pH 7 the distance to the threshold for a passive state is increased in the Pourbaix diagram for $\mathrm{Mg}$, the $\mathrm{pH}$ conditions local to the cathodic site may be sufficient to also encourage such formation (see Equation 3.5). Although, these favourable compounds may not form as extensively as they may have for a pH 10 electrolyte. The Pourbaix diagram corresponding to Al (Figure 1.7) however indicates that passive $\mathrm{Al}_{2} \mathrm{O}_{3}$ should exist at both $\mathrm{pH} 7$ and $\mathrm{pH} 10$ conditions, the presence of which is understood to have a positive impact on corrosion behaviour [88], [89]. Furthermore, Al-containing layered double hydroxides (LDH) have been revealed following corrosion of ZMA alloys [68], [73], [76]; these contribute to corrosion resistance whilst also exhibiting high degrees of stability at $\mathrm{pH} 12$ or greater [95].

The outlined rationale provides good indication as to why corrosion resistance was observed to improve for increasing $\mathrm{pH}$ conditions beyond that of $\mathrm{pH} 3$. The increased presence of the oxide/hydroxide compounds associated with the composition of the alloy likely reduced the rates of corrosion, justifying the enhancements in corrosion performance observed with increasing $\mathrm{pH}$ condition. The overall data set substantiated this concept, including the timelapse intervals of Figure 4.5 which demonstrated increased levels of corrosion product for the higher $\mathrm{pH}$ conditions. Moreover, these time-lapse microscopy images for the alkaline electrolyte of $\mathrm{pH} 10$ displayed an increased proximity of corrosion product to the site of anodic attack. This may be explained by the steep $\mathrm{pH}$ gradient between the anodic site and bulk solution, acting as a buffer and mediating the growth of this site.

\subsection{Conclusions}

The SVET was used to study the corrosion behaviour of a ZMA alloy according to varying $\mathrm{pH}$ conditions. SVET observed a shift in corrosion mechanism according to current density maps for $\mathrm{pH}$ 3. The pH 7 and $\mathrm{pH} 10$ conditions revealed a characteristic point anode to ring anode evolution, whereas anodic 'islands' of large surface coverage were observed at pH 3. Microstructural observation was conducted using time-lapse optical microscopy to study corrosion events in situ. The time-lapse intervals revealed that the extent of corrosive attack decreased for increasing pH levels. At pH 7-10, anodic attack initiated in the eutectic phases, with subsequent formation of localised anodic and cathodic regions, bordered by a ring of corrosion product. The $\mathrm{pH} 3$ experiment exhibited a modified corrosion mechanism, illustrating widespread anodic attack and some of the primary Zn-rich dendrites supporting cathodic 
activity. The perceived performance ranking between SVET and time-lapse microscopy data were contradictory; the extent of corrosion at $\mathrm{pH} 3$ was more pronounced according to timelapse microscopy than realised by SVET. However, the observed change in corrosion mechanism to a more generalised form may have led to a misinterpretation in results obtained by SVET. It is proposed that this mechanistic change is beyond the resolution of the SVET, which led to an underestimation in extent of corrosion activity. This was verified through gravimetric analysis, confirming the trend that corrosion resistance levels increased in line with $\mathrm{pH}$ condition. This was also supported by the remaining data. Open-circuit potential results displayed that the most negative potentials (vS SCE) corresponded to the lowest conditions of $\mathrm{pH}$. The polarisation data revealed a fall in log (I) for increasing $\mathrm{pH}$ levels, accordant with a reduction in rate of corrosion. This was accompanied by notable regions of passivity at anodic potentials at $\mathrm{pH} 7$ and $\mathrm{pH}$ 10. The improvements in corrosion performance at neutral and alkaline conditions were attributed to the enhanced presence and stability of favourable corrosion products on the alloy surface; the greatest extent of this effect was observed at $\mathrm{pH} 10$. 


\section{Chapter 5 The impact of dosed sodium phosphate into solution on the corrosion behaviour of a zinc-magnesium-aluminium alloy}

\subsection{Introduction}

There are multiple drivers behind the ever-present development of coating systems and the galvanising alloys they normally incorporate; the steel manufacturing business is tremendously competitive and so innovating to minimise cost outlay is key. However, the lifetime of the product itself is also crucial and therefore, corrosion performance is equally, if not more, important. A component of ensuring the lifetime of a galvanised steel product is through means of corrosion inhibition. Chromates have long been considered as the benchmark corrosion inhibitor, being utilised as conversion coatings or corrosion inhibitor pigments in the form of zinc yellow within industry. Despite the efficacy of these compounds for such a task, they have a drawback in their hazardous and toxic nature [13]-[18]. Consequently, chromate use for such applications is restricted under REACH legislation within the European Union and will be phased out by 2019. Phosphates are recognised as suitable alternatives to chromates, and within industry are readily utilised in their place as constituents of conversion coatings, paint formulation pigments, or as soluble corrosion inhibitors [11], [20]. Phosphate compounds are realised to protect typically by means of anodic inhibition [20], [21], [27], provided they are in an oxygen-containing environment. However, they have also been found to act as cathodic inhibitors through reaction with metal ions in solution, forming insoluble surface precipitates. With the addition of calcium ions, this was found to occur within cathodic areas, impeding the ability of oxygen to reach the metal surface [22], [23]. The relevancy of phosphates and their incorporation as inhibitors in industry is the reason that sodium phosphate has been selected for this study. The altered corrosion mechanisms that provide ZMA alloys with their higher degree of corrosion resistance, may also shift the efficacy of phosphates as a corrosion inhibitor, and must be studied. As such, an aim of this chapter is to characterise any performance changes resulting from phosphate additions in conjunction with a ZMA class of alloy, and to clarify the underlying mechanisms involved. This portion of work has been published (Appendix 1) and therefore, the realisations from that same work will be presented in this chapter.

This chapter focuses on a single ZMA alloy, specifically ZMA 2, due to the combination of balanced performance (as documented in the previous chapter) and its relevance from an industrial perspective; the alloy composition is detailed in 3.2.1. The effectiveness of sodium 
phosphate on ZMA was examined using scanning vibrating electrode technique (SVET) to resolve localised corrosion features and assess relative corrosion performance across a range of inhibitor concentrations. Time-lapse optical microscopy was used to study these parameters on a microstructural level in situ, and to also enable the examination of the associated corrosion mechanisms of sodium phosphate. These corrosion measurements were supported by potentiodynamic polarisation testing in conjunction with a potentiostat, to expand on any findings. Scanning electron microscopy (SEM) coupled with energy-dispersive X-ray spectroscopy (EDS) was employed for post-corrosion surface analysis.

\subsection{Experimental}

\subsubsection{Metallic specimens}

The details of the selected ZMA alloy composition for this chapter of work are detailed in Table 5.1. This sample alloy was supplied by the French Corrosion Institute, in the as-manufactured state from a commercial producer.

Table 5.1 Composition of the selected zinc-magnesium-aluminium alloy coating

\begin{tabular}{|c|c|c|c|c|}
\hline Sample & Zn (wt\%) & $\mathrm{Mg}(w t \%)$ & $\mathrm{Al}(w \mathrm{t} \%)$ & $\begin{array}{c}\text { Coating thickness } \\
(\mu \mathrm{m})\end{array}$ \\
\hline ZMA 2 & 96 & 2 & 2 & 10 \\
\hline
\end{tabular}

5.2.2 Sample preparation

As given in 2.1.2.1, a sheet of sample alloy was guillotined into $20 \mathrm{~mm} \times 20 \mathrm{~mm}$ coupons, followed by mounting, polishing to a $1 \mu \mathrm{m}$ surface finish, and a cleaning procedure using distilled water and ethanol. A 2\% Nital etchant solution was used to reveal the microstructure, followed by the prior cleaning procedure. To perform SVET experimentation, adhesive PTFE tape was used to mask the sample surface, excluding an area of $c a .10 \mathrm{~mm} \times 10 \mathrm{~mm}$ exposed for study. A largely similar masking procedure was followed for time-lapse microscopy experimentation, whereby instead a circular feature of approximately $0.785 \mathrm{~mm}^{2}$ area $(1 \mathrm{~mm}$ diameter) was exposed. Further electrochemical polarisation testing involved preparations described in 2.6; samples were removed from their mounts prior to masking were subsequently fitted securely in the custom-made sample holder, revealing an area of $0.95 \mathrm{~mm}^{2}$ exposed.

\subsubsection{Experimental methods}

Any differences observed in corrosion performance and localised mechanisms were established in part, using SVET, performing scans according to the concentration range of inhibitor additions. This is provided by SVET in the form of spatially-resolved maps, visually representing 
surface corrosion events, as well as data manipulation leading to a value of semi-quantitative metal loss and area-averaged anodic current density plots. SVET experimentation was performed using samples of ZMA 2 under immersion conditions, specifically in a baseline $1 \%$ $\mathrm{w} / \mathrm{v} \mathrm{NaCl}$ electrolyte adjusted to $\mathrm{pH}$ 7. Over the 24-hour experimental time-frame, samples were allowed to freely corrode. All samples were scanned immediately upon experimental onset, and hourly therein until the close of experiment. The SVET apparatus, operation, and calibration using the two-compartment cell is described in detail in 2.2. To maintain the accuracy of results, a minimum of two repeat scans were performed for each parameter studied.

The sodium phosphate additions were studied at a range of different concentrations to ascertain their impact on ZMA 2. The inhibitor additions were dosed into the electrolyte solution in the range of $1 \times 10^{-4}$ up to $1 \times 10^{-2} \mathrm{~mol} \mathrm{dm}^{3}$ and were adjusted to $\mathrm{pH} 7$. The use of sodium dichromate dihydrate was also studied using SVET, to act as a reference indicator from a known high-performance inhibitor; this was again via dosing of the baseline solution, but using a single concentration of $1 \times 10^{-4} \mathrm{~mol} \mathrm{dm}^{-3}$ and subsequent adjustment to $\mathrm{pH} 7$. In addition to SVET, time-lapse optical microscopy was used to study the experimental parameters within this body of work. This iteration of the technique enables the study of corroding samples under immersion conditions in situ, and at a microstructural level. Following relevant preparation, samples were fitted to the glass tank, subsequently immersed in the $1 \% \mathrm{NaCl}$ electrolyte, and the microscope lens positioned for image capture. Digital images were captured successively in 2-minute intervals over the course of the experiment. The operation of the equipment is explained further in 2.3. The experiments were initiated in the baseline electrolyte in the first instance; following the formation of a distinctive anodic site, the solution was subsequently dosed with the nominated inhibitor concentration. Electrochemical tests, taking the form of potentiodynamic polarisation curves were all conducted versus a saturated calomel electrode (SCE). These tests ranged between approximately $-1.2 \mathrm{~V}$ and $-0.90 \mathrm{~V} v \mathrm{~S}$ SCE, although not all tests reached these extremities; these polarisation tests were conducted at a sweep rate of 1 $\mathrm{mV} \mathrm{s}^{-1}$. Further analysis in the form of metal loss values was also calculated from polarisation data using Tafel extrapolation. This form of electrochemical testing is further detailed in 2.6. Surface analysis using SEM-EDS was also conducted post-corrosion. 


\subsection{Results and discussion}

5.3.1 Study of sodium phosphate as an inhibitor on the behaviour of a ZMA alloy using SVET

The SVET was used to study the relative performance of sodium phosphate as an inhibitor addition over a series of concentrations. The particular concentrations studied were $1 \times 10^{-4} \mathrm{~mol}$ $\mathrm{dm}^{-3}, 1 \times 10^{-3} \mathrm{~mol} \mathrm{dm}^{-3}$, and $1 \times 10^{-2} \mathrm{~mol} \mathrm{dm}^{-3} \mathrm{Na}_{3} \mathrm{PO}_{4}$ in a baseline $1 \% \mathrm{w} / \mathrm{v} \mathrm{NaCl}$ electrolyte neutralised to $\mathrm{pH}$ 7. As given in Figure 5.1, the chart of metal loss values measured by SVET are displayed according to the range of concentrations. The chart demonstrated a definitive trend exhibiting a significant fall in measured metal loss following the progressive additions of sodium phosphate into solution.

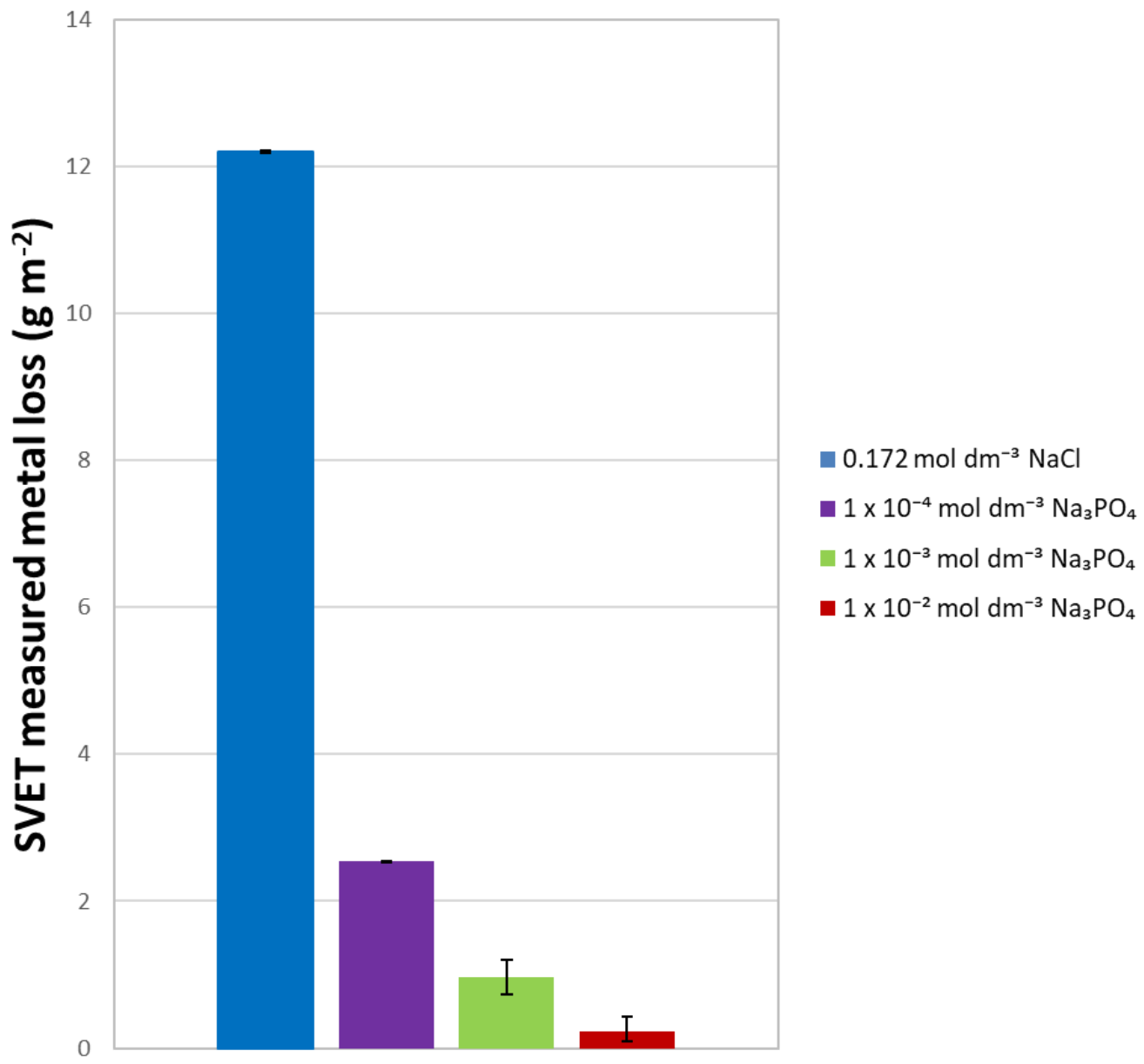

Figure 5.1 SVET estimated metal loss over 24-hour experiment time for ZMA 2 in $1 \% \mathrm{NaCl} \mathrm{pH} 7$ including the range of sodium phosphate inhibitor additions

This data was extracted into Table 5.2, displaying the inhibition efficiency for each studied inhibitor concentration. The introduction of $\mathrm{Na}_{3} \mathrm{PO}_{4}$ enabled a progressive reduction in metal loss coinciding with improvements in inhibition efficiency. This data highlighted the premise of sodium phosphate as an effective corrosion inhibitor for a ZMA 2 alloy. 
Table 5.2 Comparison of inhibition efficiency for sodium phosphate additions as acquired by

\begin{tabular}{|c|c|c|}
\hline $\begin{array}{c}\text { Sodium phosphate } \\
\text { concentration }\left(\mathrm{mol} \mathrm{dm}^{-3}\right)\end{array}$ & $\begin{array}{c}\text { SVET metal loss (g } \\
\left.\mathrm{m}^{-2}\right)\end{array}$ & Inhibition efficiency \\
\hline 0 & 12.20 & N/A \\
\hline $1 \times 10^{-4}$ & 2.54 & $79 \%$ \\
\hline $1 \times 10^{-3}$ & 0.97 & $92 \%$ \\
\hline $1 \times 10^{-2}$ & 0.23 & $98 \%$ \\
\hline
\end{tabular}

The current density maps recorded using SVET corresponding to the lowest and intermediate concentrations of sodium phosphate inhibitor have been plotted in Figure 5.2. The maps pertaining to column A displayed that some weak anodic activity was present at hour 1 . Progression of the experiment to hour 12 revealed continued anodic activity, in the form of larger anodic islands and point anodes, both of which were weak in intensity. At hour 24, the larger islands of weak anodic activity were revealed as persistent, together with the formation of fresh point anode formation; the fresh anodic sites were comparatively greater in intensity, with a single anode highlighting this to the largest extent. In the case of the intermediate inhibitor addition $\left(1 \times 10^{-3} \mathrm{~mol} \mathrm{dm}^{-3}\right)$ of column $B$, the maps demonstrated little activity by hour 1. At hour 12 , clusters of moderate intensity point anodes were visible, exhibiting some level of coalescence at this stage. The final scan of hour 24 presented the continued coalescence of these anodes, instead forming as islands of anodic activity. Their intensity was observably diminished in comparison with hour 12 , including that of the fresh anodic sites. The highest concentration of $1 \times 10^{-2} \mathrm{~mol} \mathrm{dm}^{-3}$, given in column A of Figure 5.3, displayed little perceivable anodic activity at hour 1 . This was also the case for hour 12 up until hour 24. This final scan revealed the formation of a focal point anode, with no other anodic sites discerned on the surface. Due to the understood effectiveness of chromate as a corrosion inhibitor, a $1 \times 10^{-4} \mathrm{~mol}$ $\mathrm{dm}^{-3} \mathrm{Na}_{2} \mathrm{Cr}_{2} \mathrm{O}_{7}$ SVET scan has been included in column $\mathrm{B}$; this was to indicate the performance of sodium phosphate relative to chromate. As suggested by the entirety of the SVET maps included, little activity could be determined as a result of the chromate addition, highlighting the effectiveness of this inhibitor system. 
A

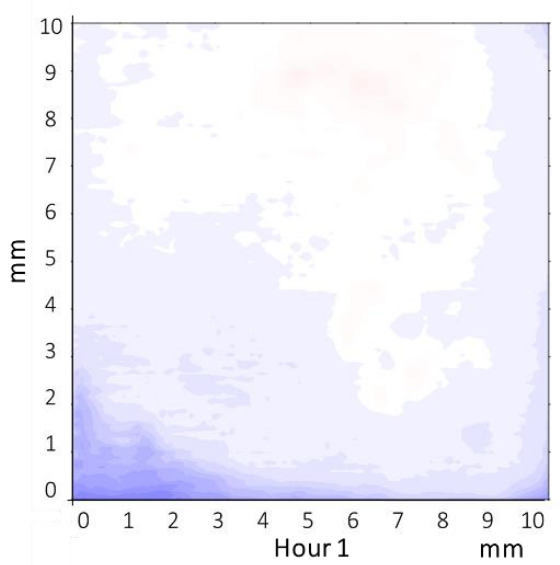

$\mathrm{Am}^{-2} \operatorname{H}_{-2}^{6}$
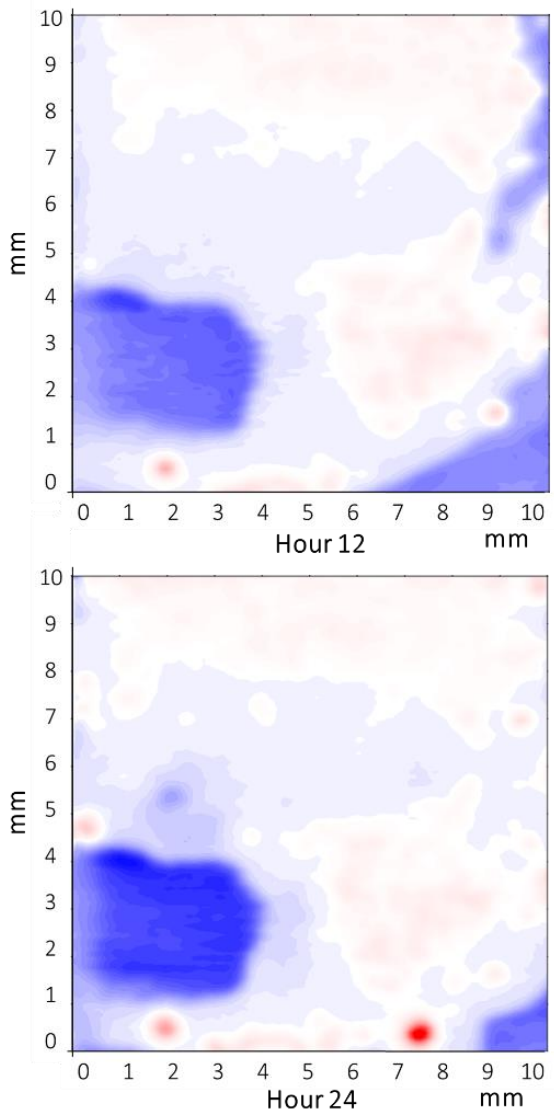

B
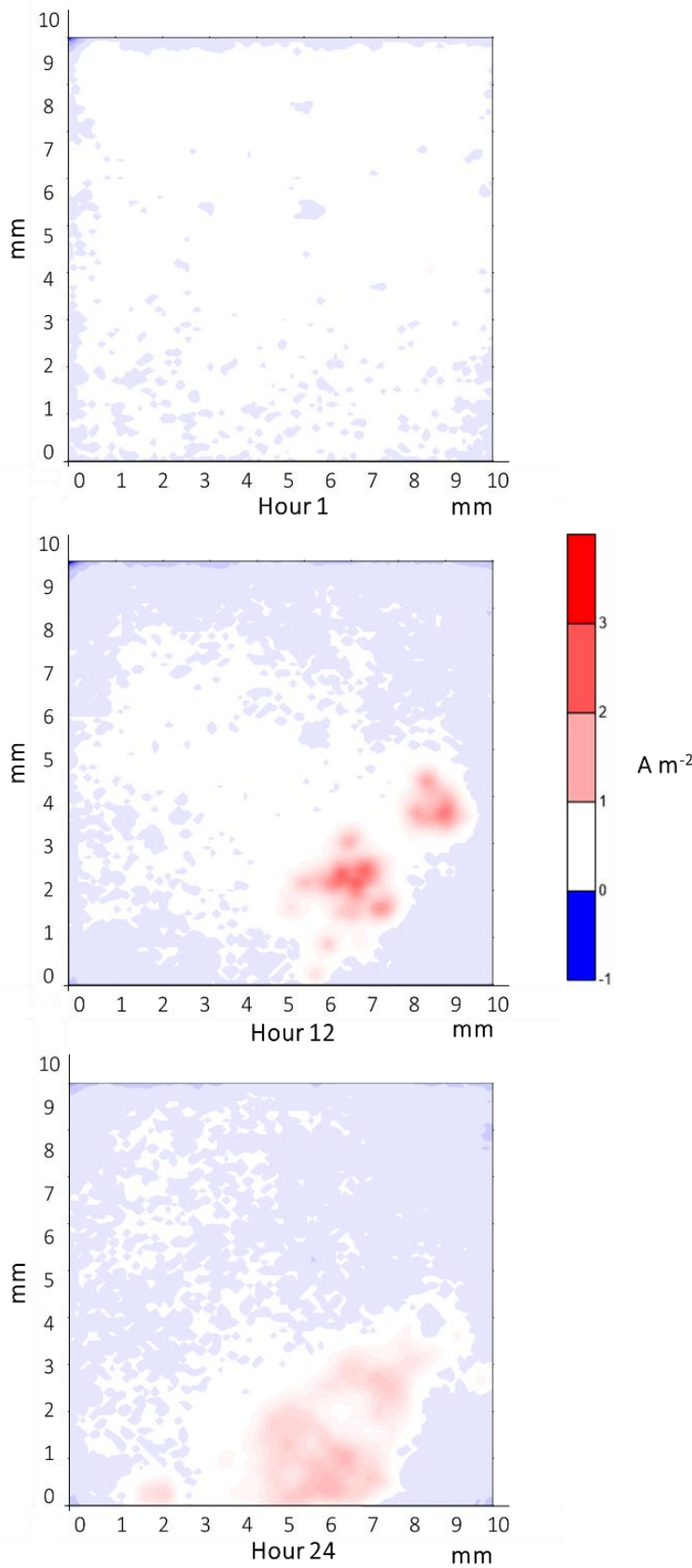

Figure 5.2 SVET-measured current density maps of ZMA 2 in $1 \% \mathrm{NaCl} \mathrm{pH} 7$ electrolyte for A) $1 \mathrm{x}$ $10^{-4} \mathrm{~mol} \mathrm{dm}^{-3}$, and B) $1 \times 10^{-3} \mathrm{~mol} \mathrm{dm}^{-3} \mathrm{Na}_{3} \mathrm{PO}_{4}$ 
A
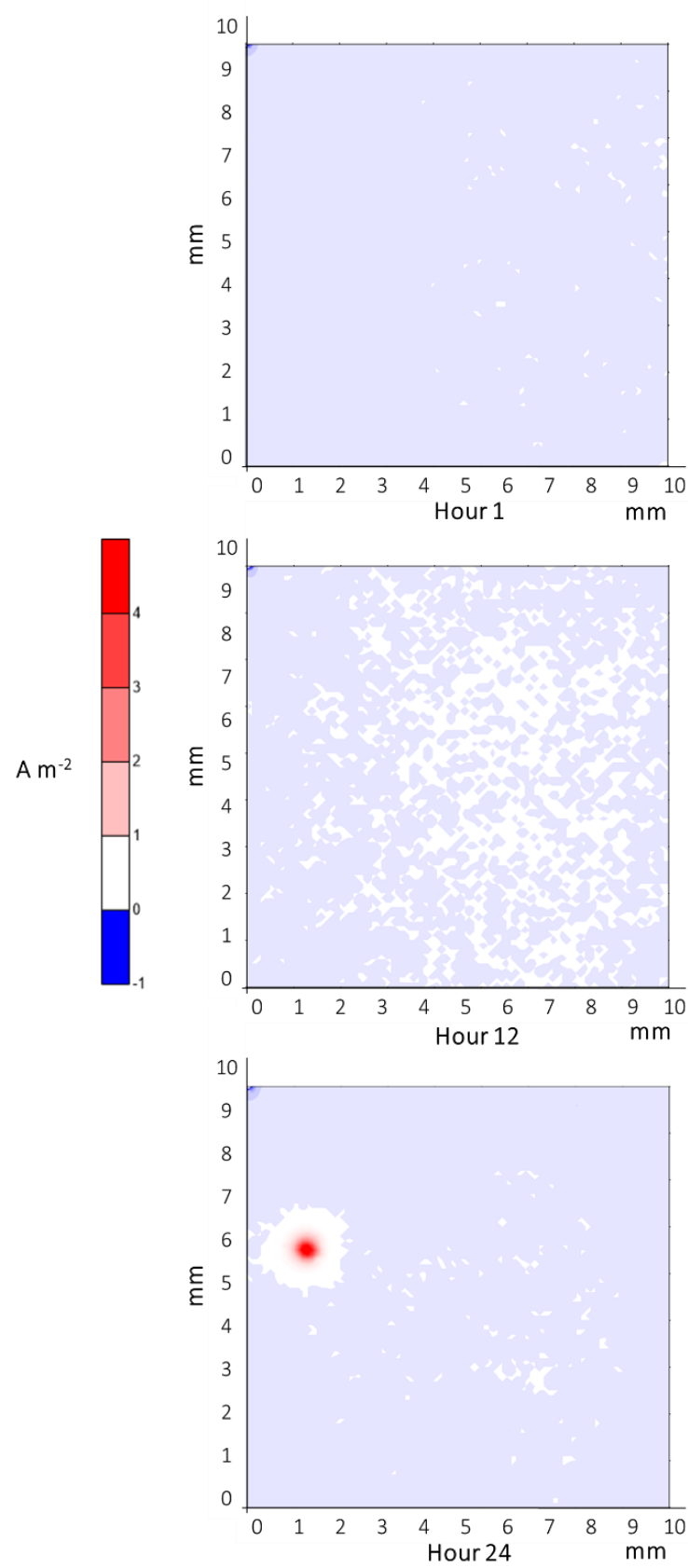

B
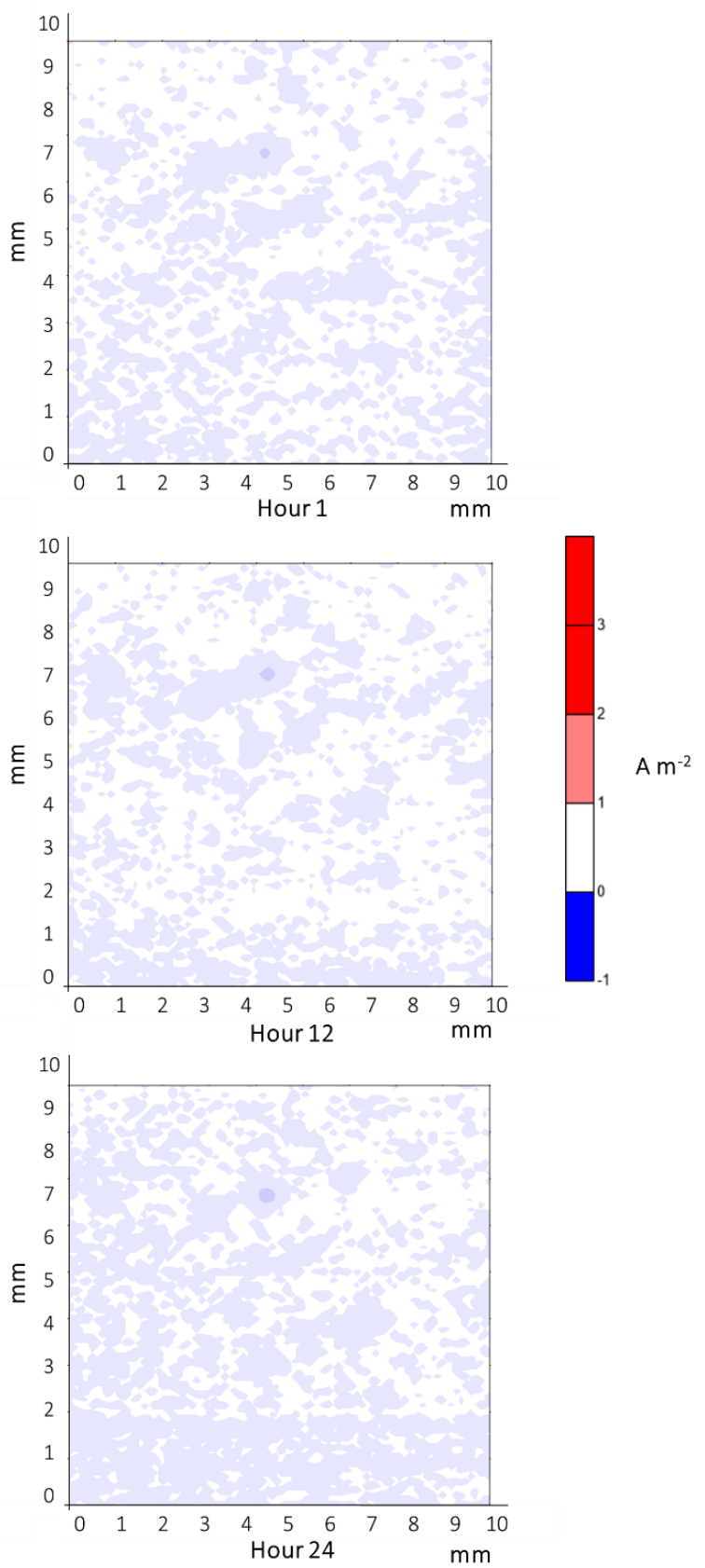

Figure 5.3 SVET-measured current density maps of ZMA 2 in $1 \% \mathrm{NaCl} \mathrm{pH} 7$ electrolyte for A) $1 \mathrm{x}$ $10^{-2} \mathrm{~mol} \mathrm{dm}^{-3} \mathrm{Na}_{3} \mathrm{PO}_{4}$, and B) $1 \times 10^{-4} \mathrm{~mol} \mathrm{dm}^{-3} \mathrm{Na}_{2} \mathrm{Cr}_{2} \mathrm{O}_{7}$

The SVET maps of Figure 5.2 displayed that the addition of sodium phosphate prevented the evolution of point anodic sites into ring anode profile observed for the uninhibited counterpart, given in Figure 3.11. Increasing the concentration of phosphate inhibitor to $1 \times 10^{-3} \mathrm{~mol} \mathrm{dm}^{-3}$ slowed the rate of anodic growth, limiting the extent of anodic evolution and surface coverage. A progressive addition of the phosphate inhibitor given in Figure 5.3 A reaffirmed this effect, evidencing a largely restricted formation of anodic activity over the experimental time-frame. This trend demonstrated that a restriction in onset and growth of anodic activity corresponded 
to an increase in sodium phosphate addition. Whilst the SVET data suggested that the phosphate-based inhibitor was highly effective, the chromate addition was more effective even at a concentration two orders of magnitude less than that of phosphate. This comparison is further demonstrated in Figure 5.4, highlighting the performance differences between the two inhibitor compounds. The SVET anode analysis of Figure 5.5 indicated a gradual rate of growth at the $1 \times 10^{-4} \mathrm{~mol} \mathrm{dm}^{-3}$, but for a larger feature corresponding to the anodic front. The intermediate sodium phosphate concentration offered a more rapid rate of growth for the point anodes present, of which were observed to merge with one another; this explains the larger growth rate observed at this concentration. This also encompassed the majority of the anodic activity on the surface. At the highest concentration, any anodic formation was delayed until the latter stages of the experiment; this was only seen to diminish in size overall, demonstrating the positive impact associated with this concentration.

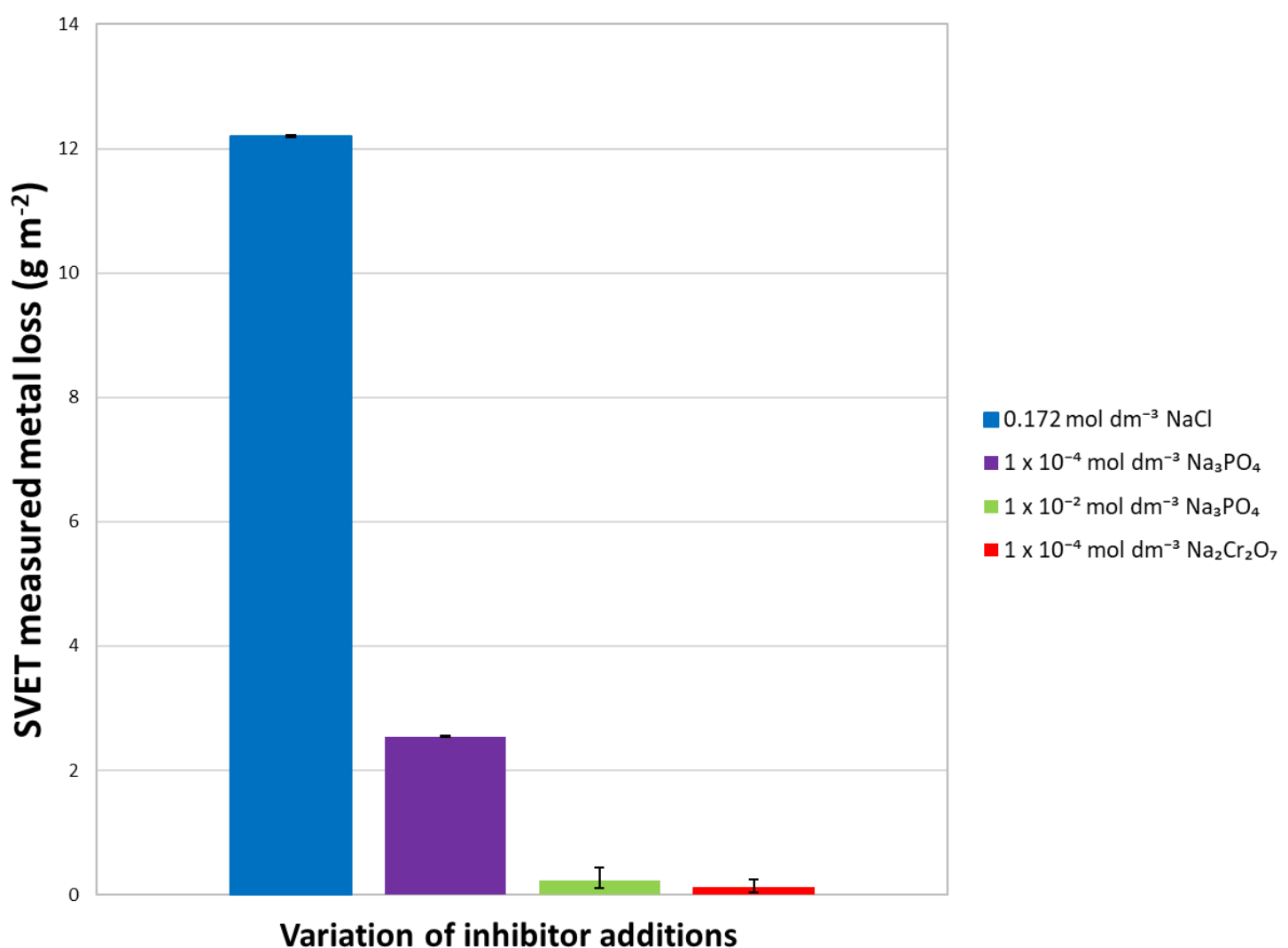

Figure 5.4 SVET estimated metal loss over 24-hour experiment for ZMA 2 in $1 \% \mathrm{NaCl} \mathrm{pH} \mathrm{7,}$ including sodium phosphate and sodium dichromate inhibitor additions 


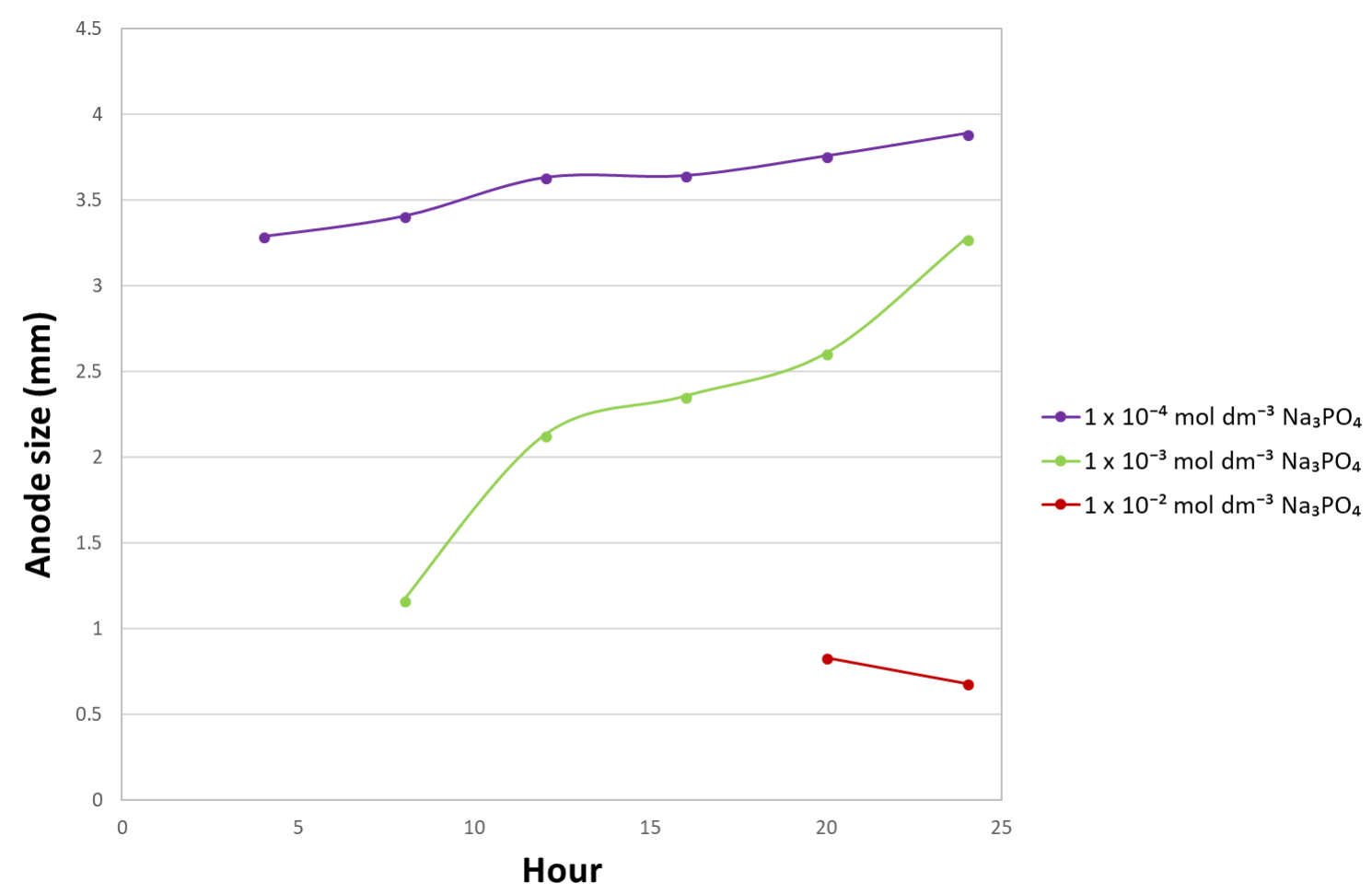

Figure 5.5 Plot of SVET anode size as a function of time for the three different sodium phosphate inhibitor species

The plot of $\mathrm{Ja}_{\mathrm{t}}$ values as a function of time for the different concentrations of sodium phosphate are given in Figure 5.6. At $1 \times 10^{-4} \mathrm{~mol} \mathrm{dm}^{-3}$, the curve exhibited very low levels of activity before hour 4, which displayed a sharp rise in Ja. This was observed to fall and gradually stabilise between hours 8-17, rising towards the tail end of the experiment. The next concentration interval increased beyond hour 1 and remained near to these values for multiple hours. The measured $\mathrm{Ja}_{\mathrm{t}}$ then reduced, exhibiting a stable and gradual fall until the end of the experiment. The highest inhibitor concentration enabled a consistently minute magnitude of activity, only displaying appreciable anodic-going activity at hour 19. At hour 20 the values corresponding to anodic activity noticeably climbed, but once again diminished in the closing hours of the experiment overall. As depicted by the plot, the addition of sodium phosphate to the electrolyte had an appreciable effect on the reduction of anodic-going current density values at any of the concentrations. This fully corroborates the overall SVET data set. Whilst the intermediate concentration did not delay the onset of anodic activity to the same extent as the lower concentration counterpart $\left(1 \times 10^{-4} \mathrm{~mol} \mathrm{dm}^{-3}\right)$, the values offered greater stability and a lower magnitude on the whole. The highest concentration of $1 \times 10^{-2} \mathrm{~mol} \mathrm{dm}^{-3} \mathrm{Na}_{3} \mathrm{PO}_{4}$ however showed a largely interrupted onset of appreciable corrosion activity until hour 19; a moderately increasing $\mathrm{Ja}_{\mathrm{t}}$ value followed, which waivered towards the end of experiment. This may indicate a performance breakdown in light of the chromate addition included, which instead exhibited a 
great degree of stability beyond initial hours of experimentation. This provided evidence of a downturn in performance for sodium phosphate even at the higher concentration in the latter stages, as demonstrated from the point anode formation in Figure 5.3 A.

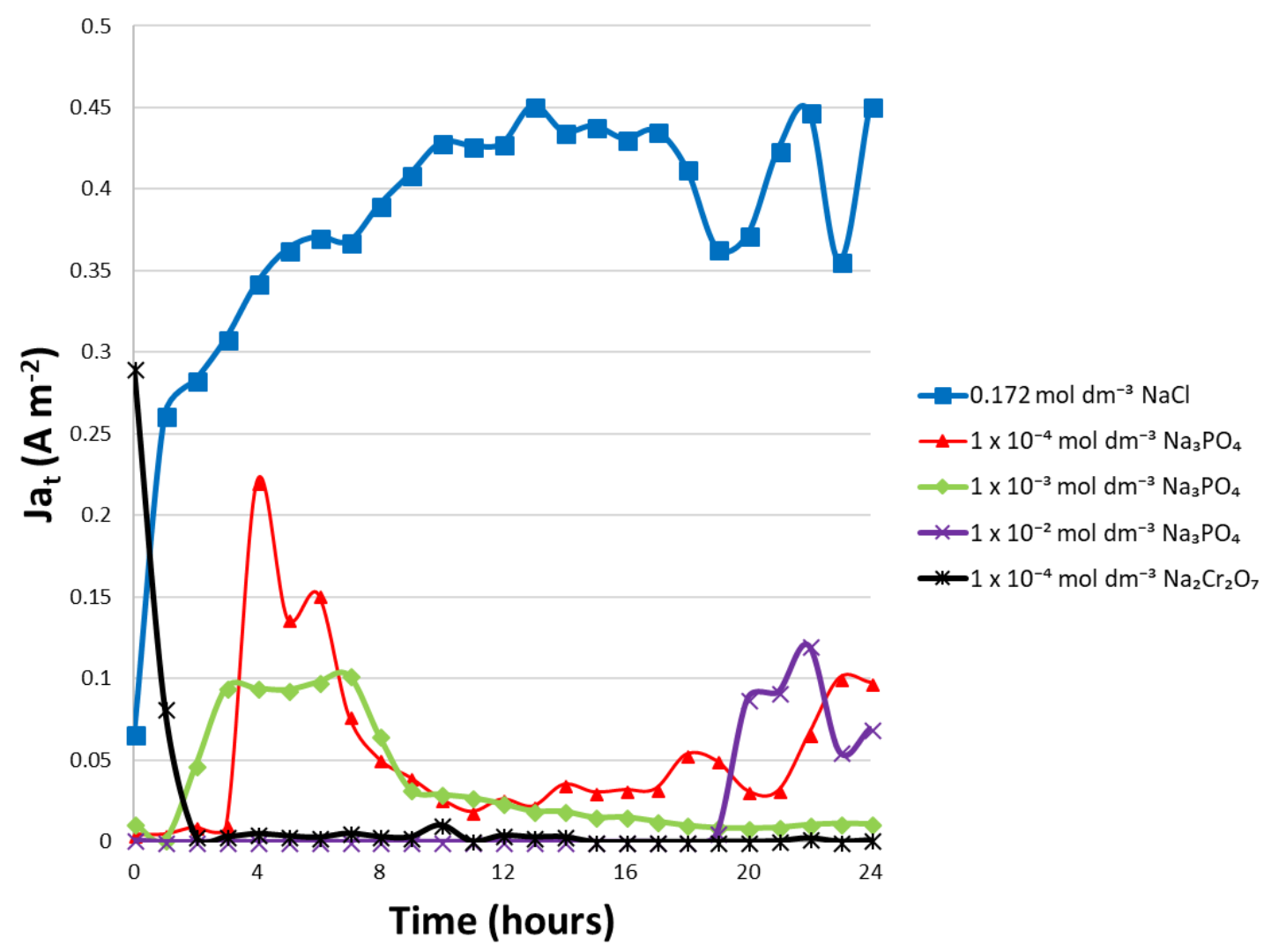

Figure 5.6 The area-averaged anodic current density $\left(\mathrm{Ja}_{\mathrm{t}}\right)$ plotted as a function of time for ZMA 2 alloy in $1 \% \mathrm{NaCl} \mathrm{pH} \mathrm{7,} \mathrm{encompassing} \mathrm{the} \mathrm{range} \mathrm{of} \mathrm{studied} \mathrm{inhibitor} \mathrm{additions}$

5.3.2 Examination of the effects of sodium phosphate inhibitor additions on ZMA 2 using time-lapse optical microscopy

The microstructural examination of ZMA samples was performed using time-lapse optical microscopy, considering the three different concentrations of sodium phosphate inhibitor. This involved allowing a sample to freely corrode in an uninhibited solution until appreciable anodic activity was established. The $1 \% \mathrm{NaCl}$ electrolyte was then dosed with a designated concentration of inhibitor, so as to more easily distinguish between the action of conventional corrosion and that incorporating any inhibitor species. Corresponding to Figure 5.7-5.8, image A was captured at the onset of the experiment, followed by image B taken immediately preceding addition of the inhibitor. Images $C$ and $D$ are those captured at 6-hour intervals following inhibitor introduction into the electrolyte. In the case of Figure 5.9, image A represents the image captured directly prior to the addition of phosphate inhibitor in order to demonstrate the inhibitor action more clearly; the subsequent images (B-D) were captured at 6-hour intervals. 
The images in Figure 5.7 demonstrated the clear formation and growth of an anodic site on the sample surface, initiating in the eutectic regions. Image B revealed the presence of a band of corrosion product appearing diagonally across the surface, and the phosphate inhibitor was introduced immediately following this interval. Images C-D illustrated that the growth of the anodic site developed in tandem with the corrosion product, the latter of which accumulated over the remaining intervals, extending towards the region of cathodic activity. Comparative to the uninhibited counterpart time-lapse experiment examined in Figure 3.11, the extent of anodic attack was visually reduced as a result of the phosphate inhibitor addition. However, whilst phosphate is normally considered to act as an anodic inhibitor [20], [21], the mechanism with which it acted to provide corrosion resistance was not entirely clear from Figure 5.7 alone.

The mechanism associated with this inhibitor system was shown more clearly for the intermediate concentration of $1 \times 10^{-3} \mathrm{~mol} \mathrm{dm}^{-3}$, given in Figure 5.8. The development of a focal anodic feature was demonstrated between images $A$ and $B$, accompanied by the corrosion product at a given distance from the anode. Upon image $C$, the dosing of the inhibitor had a clear effect in the vicinity of the anode, which exhibited little growth from the prior interval. A darkened appearance in the anodic region and surrounding area was present, with the cathodic area visually unaffected. This continued into image $D$, with an enriched and darkened area encompassing the anodic activity; the site of cathodic activity remained unchanged. The observed darkening of the surface was likely a surface precipitate resultant of the inhibitor species, as it was not present prior to dosing of the electrolyte. The fact that this surface film precipitated proximal to the anode provided a good indication of the phosphate species acting as an anodic inhibitor. However, this precipitation was not obvious for any particular phase, and provided general coverage in the vicinity of the anodic site.

In Figure 5.9, pertaining to the greatest addition of sodium phosphate at $1 \times 10^{-2} \mathrm{~mol}$ $\mathrm{dm}^{-3}$, a distinctive anodic region and corrosion product were present in image $A$; once again, this was initiated in the eutectic region The transition to image $B$, whereby the inhibitor was present, displayed a small extent of anodic growth accompanied by sites of precipitation distributed across the surface. The precipitates were observed at the predominant anodic region as well as at other potential anodic sites and surface blemishes; the precipitates themselves could be distinguished by the fact that they permitted the observation of the underlying microstructure, suggesting that they were of a thin disposition. Progressing into images C-D, the surface precipitates continued to form and accumulate, heavily restricting any perceivable growth of anodic activity generally focused at eutectic regions. Surplus to the 
characteristic deposition of species at potentially active sites, the formation of inhibitor-based 'filaments' were visible on the surface. The thin wire-like structures were present at active sites, but progressed in a seemingly independent fashion regardless of the exposed microstructure to which it was adsorbed. This was clarified in Video 5.3.

The time-lapse microscopy results presented a clear ranking in full agreement with the SVET data, showing that sodium phosphate was indeed an effective corrosion inhibitor. The extent of the inhibition increased according to a rise in concentration, with $1 \times 10^{-2} \mathrm{~mol} \mathrm{dm}^{-3}$ revealing considerably improved corrosion resistance. This was further evidenced in Videos 5.15.3.
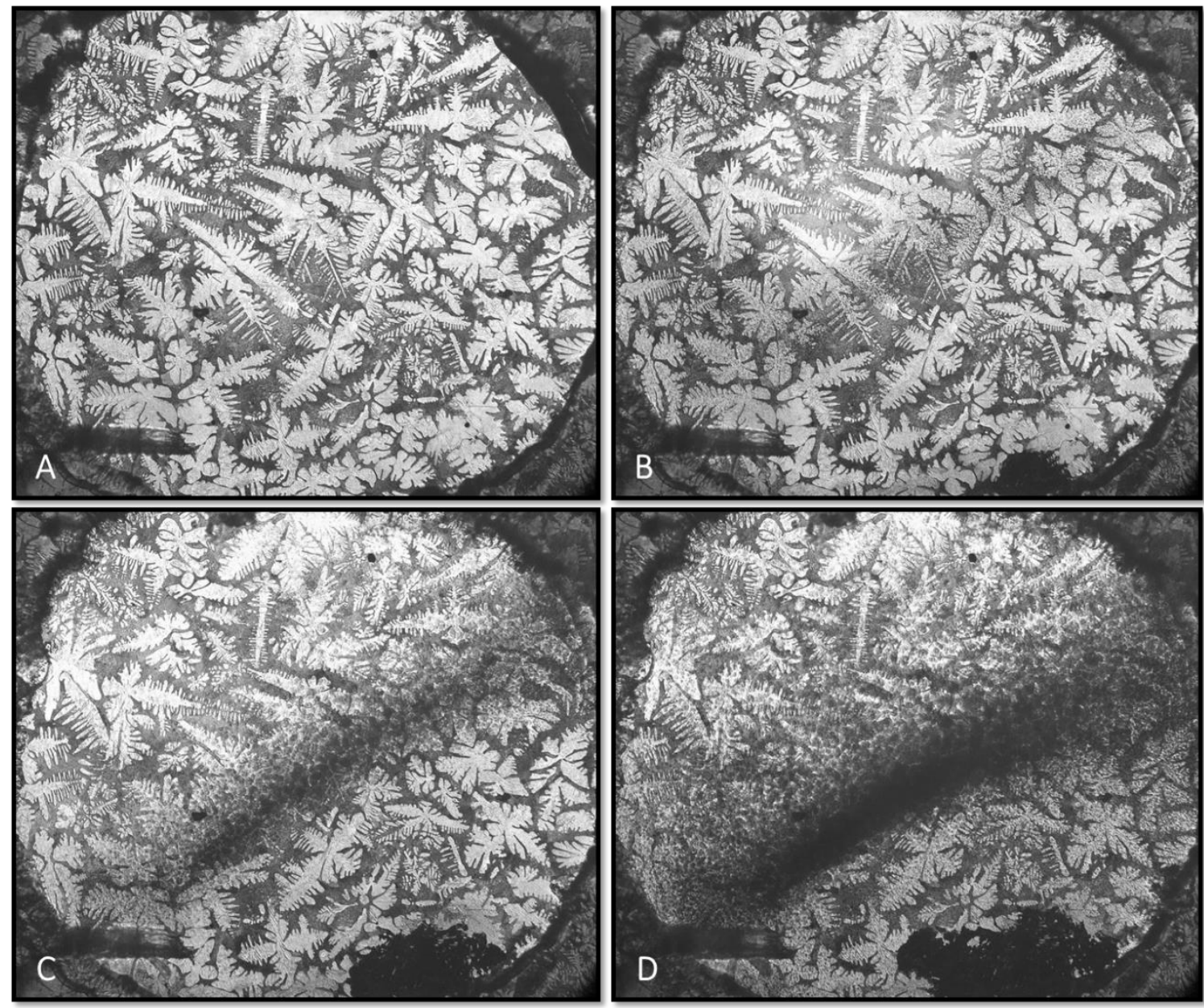

$250 \mu \mathrm{m}$

Figure 5.7 Time-lapse microscopy images of ZMA 2 captured, in a $1 \% \mathrm{NaCl}$ pH 7 electrolyte for (A-D) $1 \times 10^{-4} \mathrm{~mol} \mathrm{dm}^{-3} \mathrm{Na}_{3} \mathrm{PO}_{4}$ 

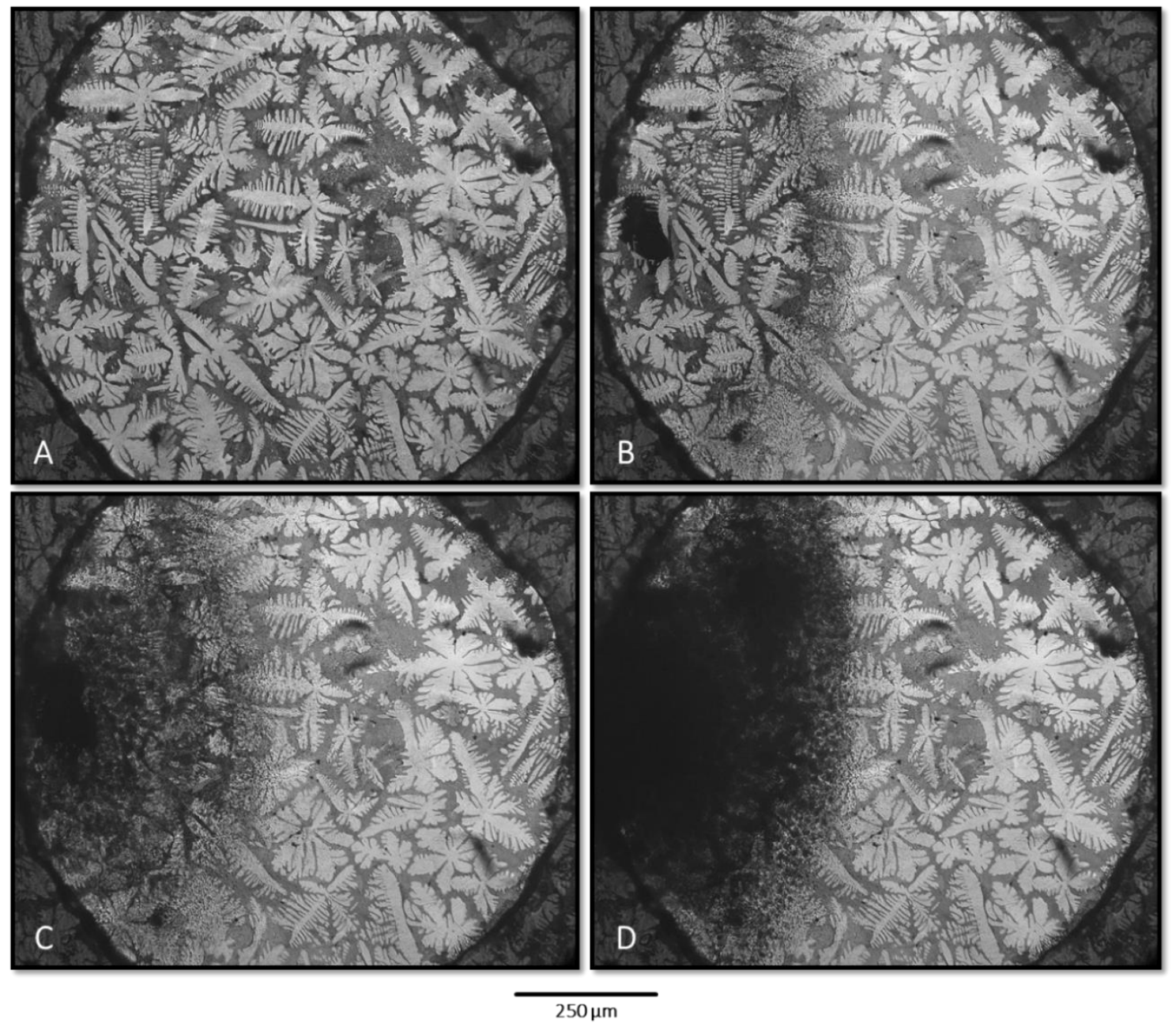

Figure 5.8 Time-lapse microscopy images of ZMA 2 captured, in a $1 \% \mathrm{NaCl} \mathrm{pH} 7$ electrolyte for (A-D) $1 \times 10^{-3} \mathrm{~mol} \mathrm{dm}^{-3} \mathrm{Na}_{3} \mathrm{PO}_{4}$ 

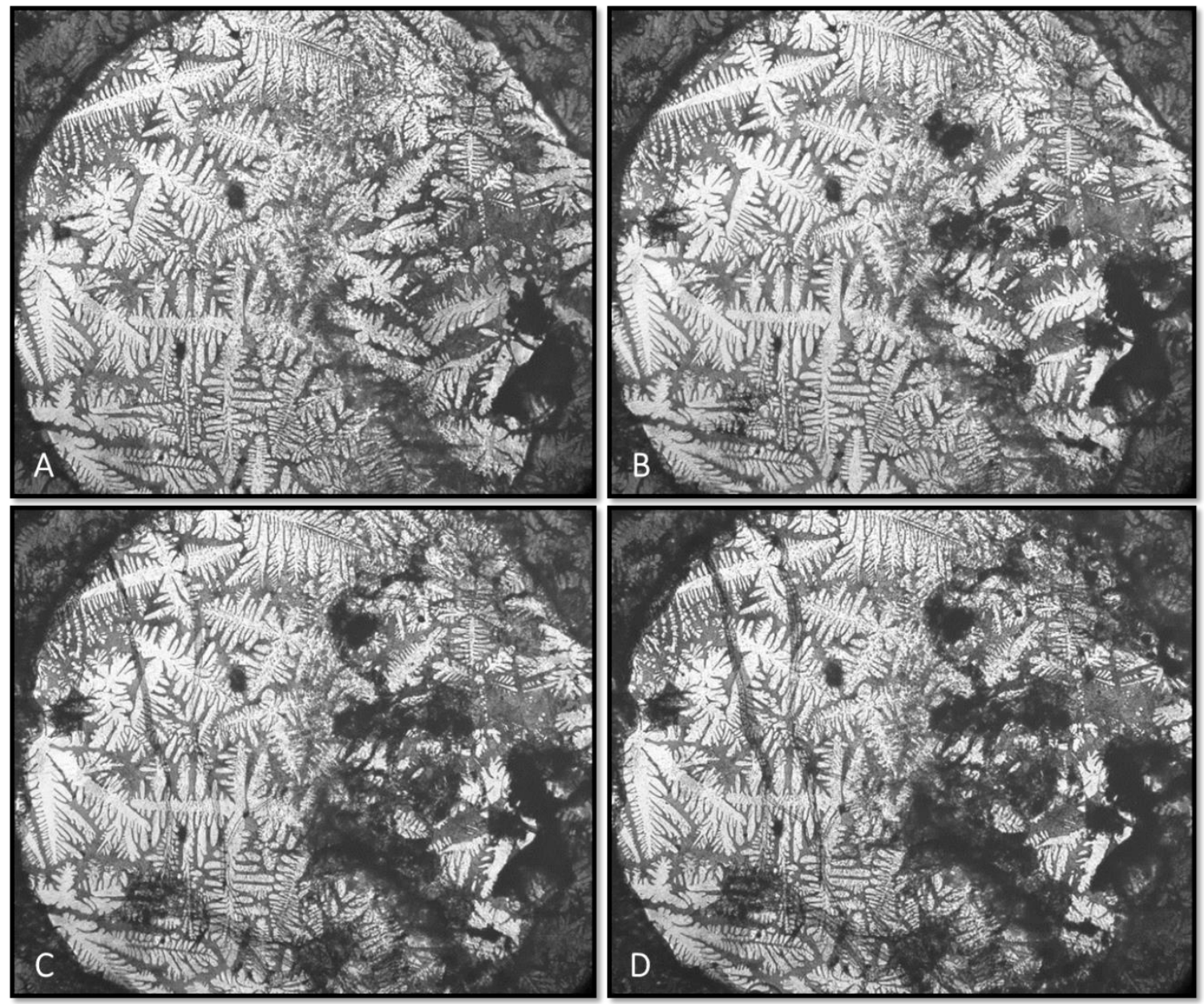

$250 \mu \mathrm{m}$

Figure 5.9 Time-lapse microscopy images of ZMA 2 captured, in a $1 \% \mathrm{NaCl}$ pH 7 electrolyte for (A-D) $1 \times 10^{-2} \mathrm{~mol} \mathrm{dm}^{-3} \mathrm{Na}_{3} \mathrm{PO}_{4}$

5.3.3 Polarisation curves per the studied sodium phosphate inhibitor additions

A set of potentiodynamic polarisation curves were completed in conjunction with a potentiostat as given in Figure 5.10. This experiment involved allowing each sample to freely corrode for approximately 80 minutes to stabilise in solution, and subsequently performing the polarisation sweep. Beginning from the most negative potential, the sweeps were conducted in the range of $-1.20 \mathrm{~V}$ vs SCE up to $-0.90 \mathrm{~V}$ vs SCE. The curves given in Figure 5.10 demonstrated that the $\mathrm{E}_{\text {corr }}$ measurements became more positive following the increase in phosphate inhibitor concentration. This coincides with the Evans diagram for anodic inhibition, plotted in Figure 1.8, exhibiting an increase in $E_{\text {corr }}$ for such systems. At $-1.00 \mathrm{~V}$ vs SCE, the values of log (I) revealed a large drop between the uninhibited sample and that including the smallest concentration of inhibitor; at this potential, the $1 \times 10^{-4} \mathrm{~mol} \mathrm{dm}^{-3} \mathrm{Na}_{3} \mathrm{PO}_{4}$ measured more than an order of magnitude reduction comparatively. This reduction continued between the minimal and intermediate $1 \times 10^{-3} \mathrm{~mol} \mathrm{dm}^{-3}$ addition, but the extent of this was not as severe. A larger fall in $\log (\mathrm{I})$ was once again visible for the highest concentration of phosphate inhibitor, exhibiting a 
value approximately two orders of magnitude less than that of the uninhibited sample. As illustrated by $1 \times 10^{-2} \mathrm{~mol} \mathrm{dm}^{-3} \mathrm{Na}_{3} \mathrm{PO}_{4}$, a large effect to the anodic branch suggestive of passivation occurred in the presence of the inhibitor, exhibiting a large restriction in anodicgoing rate relative to the uninhibited counterpart. The overall trend of the data offered the indication of an improvement in corrosion performance for increasing concentrations of sodium phosphate inhibitor additions in solution, as observed in the above data sets.

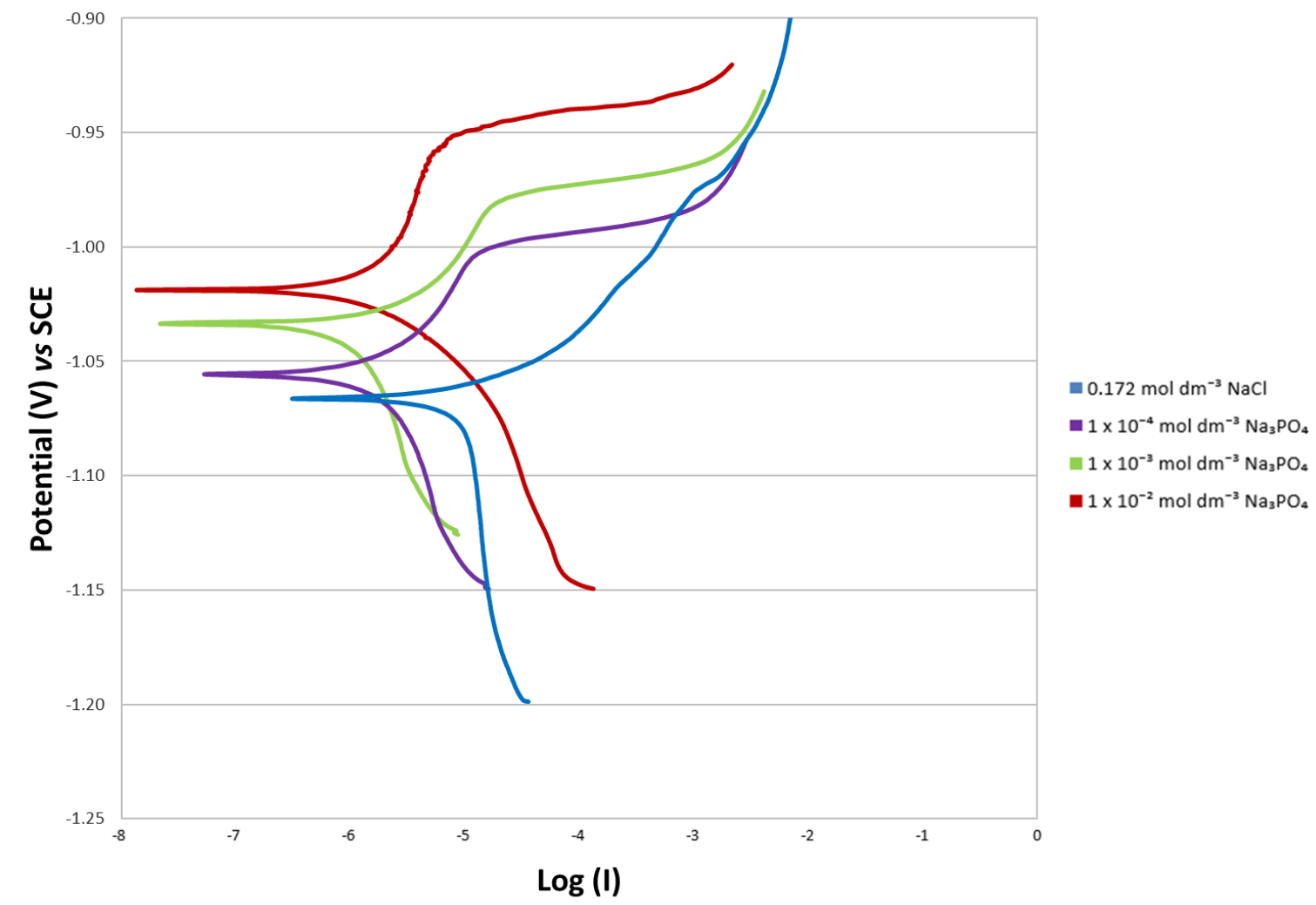

Figure 5.10 Plot of polarisation curves for the range of dosed sodium phosphate concentrations in $1 \% \mathrm{NaCl}$ electrolyte

The Tafel-extrapolated metal loss values from the polarisation data given in Table 5.3 sustained this trend; a reduction in metal loss proportionally similar to SVET was observed between the control experiment and that containing $1 \times 10^{-4} \mathrm{~mol} \mathrm{dm}^{-3}$ of the inhibitor. However, the values realised between the two methods of metal loss approximations were very much different, with Tafel data largely underestimating relative to SVET data. Nevertheless, the Tafel data demonstrated that metal loss diminished for increasing concentrations of phosphate-based corrosion inhibitor. Whilst the Tafel values were divergent to those obtained through SVET, the performance ranking between the different concentrations remained identical. One of the reasons attributable to the observed discrepancy between metal loss values obtained by the different techniques may be based on the typical localised corrosion mechanism apparent for this class of alloy; it is understood that the determination of corrosion rates via Tafel 
extrapolation is less accurate for localised corrosion than it is for generalised corrosion mechanisms [96]. Accordingly, that may have been a contributing factor.

Table 5.3 Metal loss values using SVET and Tafel-extrapolation

\begin{tabular}{|c|c|c|}
\hline $\begin{array}{c}\text { Sodium phosphate } \\
\text { concentration }\left(\mathrm{mol} \mathrm{dm}^{-3}\right)\end{array}$ & $\begin{array}{c}\text { SVET metal } \\
\text { loss }\left(\mathrm{g} \mathrm{m}^{-2}\right)\end{array}$ & $\begin{array}{c}\text { Tafel-derived metal } \\
\text { loss }\left(\mathrm{g} \mathrm{m}^{-2}\right)\end{array}$ \\
\hline 0 & 12.20 & 0.78 \\
\hline $1 \times 10^{-4}$ & 2.54 & 0.15 \\
\hline $1 \times 10^{-3}$ & 0.97 & 0.11 \\
\hline $1 \times 10^{-2}$ & 0.23 & 0.10 \\
\hline
\end{tabular}

\subsubsection{Surface analysis of corrosion inhibitor deposition using SEM-EDS}

Upon completion of a time-lapse microscopy experiment, a sample having been immersed in $1 \% \mathrm{NaCl} \mathrm{pH} 7$ electrolyte containing $1 \times 10^{-2} \mathrm{~mol} \mathrm{dm}^{-3} \mathrm{Na}_{3} \mathrm{PO}_{4}$ was utilised for post-corrosion observation. This was to enable the inspection of the surface and corrosion products consequent for the inhibitor addition. As given in Figure 5.11, image A represents the surface of the sample containing an adsorbed filament associated with the deposition of the inhibitor. Images B-D are that of the corresponding EDS data to identify the elements constituting the deposit of interest. As indicated by the EDS maps, the presence of $\mathrm{Mg}, \mathrm{P}$ and $\mathrm{O}$ were rich on the deposited filament. Accordingly, the compound may have been composed of magnesium phosphate. This can be understood due to the preferential attack of magnesium in the early stages of corrosion, permitting the formation of a complex of magnesium cations and phosphate anions. Likewise, the highly reactive nature of $\mathrm{MgZn}_{2}$ phase may have caused a similar situation for $\mathrm{Zn}$, as deposits rich in $\mathrm{Zn}, \mathrm{P}$, and $\mathrm{O}$ were also found during the SEM-EDS analysis. As the solubility of both magnesium phosphate or zinc phosphate compounds is poor, it can be realised that these would precipitate on the surface of the ZMA sample. Whilst the given data was not definitive, it provided an adequate indication of the composition of the surface precipitates. 

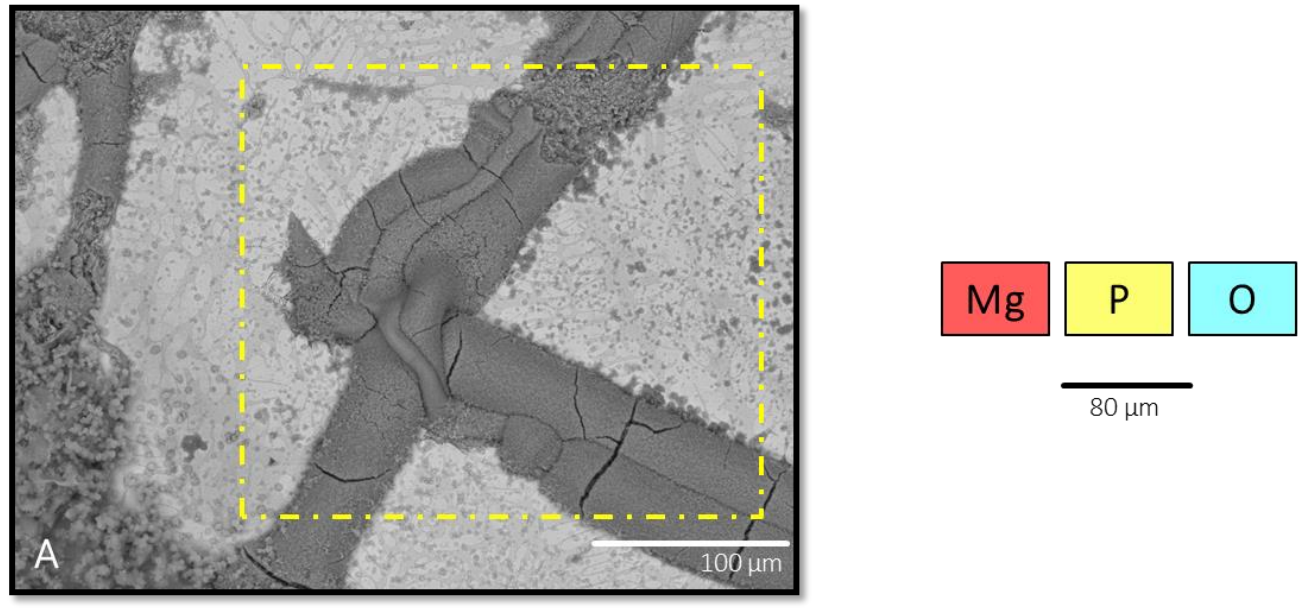

$80 \mu \mathrm{m}$
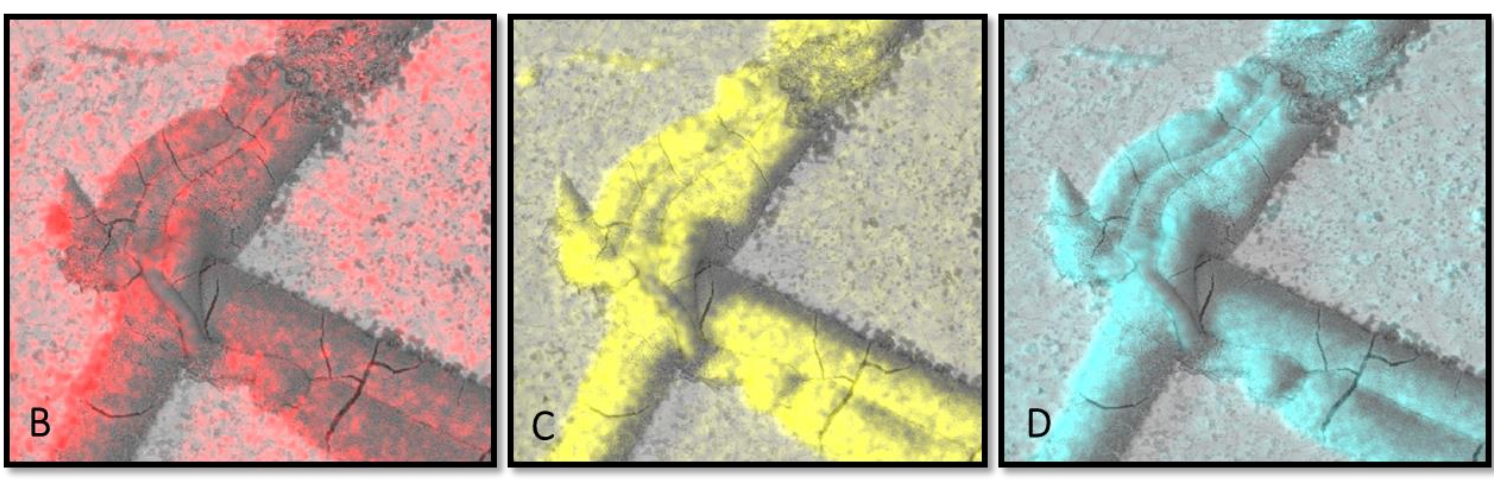

Figure 5.11 (A-D) SEM and EDS analysis of a ZMA sample following immersion in $1 \times 10^{-2} \mathrm{~mol} \mathrm{dm}^{-3}$ $\mathrm{Na}_{3} \mathrm{PO}_{4}$

\subsubsection{Calculation of solubility characteristics for the dosed sodium phosphate inhibitor}

Given that the conditions of a solution can dictate the species of phosphate that may form, this aspect must be considered. Understanding that for an anodic inhibitor addition to prove effective in the tested conditions, it must have solubility characteristics of a sparingly soluble, or insoluble nature in aqueous chloride media. These conditions would enable the precipitation of an adherent, insoluble species on the surface [97], physically shielding the anodic site and consequently regulating the rate of corrosion events. If the phosphate species has an increased solubility in such conditions, then this will not transpire and a film will not deposit, foregoing any advantageous effect on the corrosion rate. In the case of zinc phosphates, the primary species is recognised as soluble in water, whilst the secondary forms are realised to be poorly soluble [98]; it is also understood that tertiary phosphate species are insoluble [99]-[101], enabling the deposition of metal tertiary phosphates onto a substrate [97]. Conventionally, phosphate-based conversion coatings are composed of dilute phosphoric acid solutions, forming a soluble primary phosphate species via reaction between the substrate and free phosphoric acid [97]. 
For a typical ZMA alloy, both magnesium and zinc are understood to corrode, such as $\mathrm{MgZn}_{2}$ phase in the first instance. Accordingly, soluble primary zinc phosphates and magnesium phosphates would be expected to form in the presence of free phosphoric acid. However, given the $\mathrm{pK}_{\mathrm{a}}$ values for phosphoric acid of 2.0, 6.9 and 12.5 [102], as well as the $\mathrm{pH}$ of the electrolyte, the equilibrium concentration of the insoluble tertiary phosphate species $\left(\left[\mathrm{PO}_{4}{ }^{3-}\right]\right)$ can be calculated. The expression to calculate this is given in Equation 5.1, as follows [102]:

$$
\left[\mathrm{PO}_{4}^{3-}\right]=\frac{\left[\mathrm{PO}_{4}^{3-}\right]_{\text {total }}}{1+10^{(-p H+12.5)}+10^{(-2 p H+12.5+6.9)}+10^{(-3 p H+12.5+6.9+2)}}
$$

As $\left[\mathrm{PO}_{4}{ }^{3-}\right]_{\text {total }}$ from Equation 5.1 corresponds to the concentration of phosphate species present in solution, each of the three studied inhibitor concentrations can be assessed. Whilst $\mathrm{Mg}$ is realised to readily corrode on a ZMA alloy in the initial stages, literature has suggested that protective Zn-based corrosion products are prevalent [72], [73]. Additionally, both elements were observed from the SEM-EDS analysis of the filament-like precipitates, of which Mg was present in Figure 5.11. Consequently, the calculations according to Equation 5.1 consider both elements individually. To ascertain the precipitation of the insoluble $\mathrm{Zn}_{3}\left(\mathrm{PO}_{4}\right)_{2}$ and $\mathrm{Mg}_{3}\left(\mathrm{PO}_{4}\right)_{2}$ compounds, each associated solubility product $\left(K_{\mathrm{sp}}\right)$ and solubility quotient $\left(\mathrm{Q}_{\mathrm{s}}\right)$ must be known. Accordingly, the values of $Q_{s}$ are given for both zinc phosphate and magnesium phosphate in Equation 5.2 and Equation 5.3, respectively.

$$
\begin{aligned}
& Q_{s p}=\left[\mathrm{Zn}^{2+}\right]^{3} \cdot\left[\mathrm{PO}_{4}{ }^{3-}\right]^{2} \\
& Q_{s p}=\left[\mathrm{Mg}^{2+}\right]^{3} \cdot\left[\mathrm{PO}_{4}{ }^{3-}\right]^{2}
\end{aligned}
$$

The solubility product values for both $\mathrm{Zn}_{3}\left(\mathrm{PO}_{4}\right)_{2}$ and $\mathrm{Mg}_{3}\left(\mathrm{PO}_{4}\right)_{2}$ are known $\left(9.1 \times 10^{-33}\right.$ [103], and $1.00 \times 10^{-25}$ [102], respectively). Therefore, it can be understood that the insoluble tertiary species will precipitate provided that the solubility quotient exceeds that of the solubility product. Based on these outlined understandings, Microsoft Excel was used to manipulate the data and indicate whether precipitation of the insoluble phosphate species was apparent for a given concentration of divalent $\mathrm{Zn}$ or $\mathrm{Mg}$ ionic species in solution. An estimate of concentration for $\mathrm{Zn}^{2+}$ and $\mathrm{Mg}^{2+}$ was derived from the SVET-based metal loss values associated with an entire 24-hour experiment. Considering the volume of solution beneath the plane of scan height, a value of concentration per hour, taken as an average over the entire area and full experiment 
length was calculated. Whilst the values are by no means definitive due to the assumptions they are based upon, they have been used as an indicator to support other findings from this Chapter. The results for the set of calculations are given in Table 5.4 and Table 5.5 for zinc and magnesium, respectively. These indicated that at each inhibitor concentration studied, the available tertiary phosphate species in solution and free concentration of both $\mathrm{Zn}^{2+}$ and $\mathrm{Mg}^{2+}$ ions enabled the precipitation of the insoluble metal phosphate species. The extent of this indicated that precipitation increased according to higher inhibitor concentration values, fully agreeing with the data acquired from the chapter.

Table 5.4 Estimation of tertiary zinc phosphate precipitation likelihood in according to solution inhibitor concentration variation at $\mathrm{pH} 7$

\begin{tabular}{|c|c|c|c|c|c|c|}
\hline $\begin{array}{c}\text { Phosphate } \\
\text { concentration } \\
\text { in solution } \\
\left(\mathrm{mol} \mathrm{dm}^{-3}\right)\end{array}$ & $\begin{array}{c}\text { Available } \\
\text { tertiary } \\
\text { phosphate } \\
\left(\mathrm{mol} \mathrm{dm}^{-3}\right)\end{array}$ & $\begin{array}{c}\text { SVET- } \\
\text { derived } \\
{\left[\mathrm{Zn}^{2+}\right](\mathrm{mol}} \\
\left.\mathrm{dm}^{-3} \text { hour }\right)\end{array}$ & $\begin{array}{c}\text { Solubility } \\
\text { quotient }\end{array}$ & $\begin{array}{c}\text { Solubility } \\
\text { product }\end{array}$ & $\begin{array}{c}\text { Free }\left[\mathrm{Zn}^{2+}\right] \\
\text { required for } \\
\text { precipitation } \\
\left(\mathrm{mol} \mathrm{dm}^{-3}\right)\end{array}$ & $\begin{array}{c}\text { Precipitation } \\
\text { of zinc } \\
\text { phosphate }\end{array}$ \\
\hline $1 \times 10^{-4}$ & $1.76 \times 10^{-10}$ & $1.62 \times 10^{-2}$ & $1.32 \times 10^{-25}$ & $9.1 \times 10^{-33}$ & $6.64 \times 10^{-5}$ & Yes \\
\hline $1 \times 10^{-3}$ & $1.76 \times 10^{-9}$ & $6.17 \times 10^{-3}$ & $7.28 \times 10^{-25}$ & $9.1 \times 10^{-33}$ & $1.43 \times 10^{-5}$ & Yes \\
\hline $1 \times 10^{-2}$ & $1.76 \times 10^{-8}$ & $1.49 \times 10^{-3}$ & $1.03 \times 10^{-24}$ & $9.1 \times 10^{-33}$ & $3.08 \times 10^{-6}$ & Yes \\
\hline
\end{tabular}

Table 5.5 Estimation of tertiary magnesium phosphate precipitation likelihood in according to solution inhibitor concentration variation at $\mathrm{pH} 7$

\begin{tabular}{|c|c|c|c|c|c|c|}
\hline $\begin{array}{c}\text { Phosphate } \\
\text { concentration } \\
\text { in solution } \\
\left(\mathrm{mol} \mathrm{dm} \mathrm{dm}^{-3}\right)\end{array}$ & $\begin{array}{c}\text { Available } \\
\text { tertiary } \\
\text { phosphate } \\
\left.(\mathrm{mol} \mathrm{dm})^{-3}\right)\end{array}$ & $\begin{array}{c}\text { SVET- } \\
\text { derived } \\
{\left[\mathrm{Mg}^{2+}\right](\mathrm{mol}} \\
\left.\mathrm{dm}^{-3} \text { hour }\right)\end{array}$ & $\begin{array}{c}\text { Solubility } \\
\text { quotient }\end{array}$ & $\begin{array}{c}\text { Solubility } \\
\text { product }\end{array}$ & $\begin{array}{c}\text { Free }\left[\mathrm{Mg}^{2+}\right] \\
\text { required for } \\
\text { precipitation } \\
\left(\mathrm{mol} \mathrm{dm}^{-3}\right)\end{array}$ & $\begin{array}{c}\text { Precipitation } \\
\text { of } \\
\text { magnesium } \\
\text { phosphate }\end{array}$ \\
\hline $1 \times 10^{-4}$ & $1.76 \times 10^{-10}$ & $4.36 \times 10^{-2}$ & $2.57 \times 10^{-24}$ & $1.0 \times 10^{-25}$ & $1.48 \times 10^{-2}$ & Yes \\
\hline $1 \times 10^{-3}$ & $1.76 \times 10^{-9}$ & $1.66 \times 10^{-2}$ & $1.42 \times 10^{-23}$ & $1.0 \times 10^{-25}$ & $3.18 \times 10^{-3}$ & Yes \\
\hline $1 \times 10^{-2}$ & $1.76 \times 10^{-8}$ & $4.02 \times 10^{-3}$ & $2.01 \times 10^{-23}$ & $1.0 \times 10^{-25}$ & $6.85 \times 10^{-4}$ & Yes \\
\hline
\end{tabular}

\subsubsection{Evaluation of sodium phosphate inhibitor action on a ZMA 2 alloy}

The entirety of the data gathered during experimentation highlighted that sodium phosphate acted to improve the levels of corrosion resistance on a ZMA surface. The SVET data showed a reduction in measured metal loss values for increasing concentrations of inhibitor present in solution, and despite the comparative difference in magnitude of the Tafel-extrapolated values, this data reported a parallel trend. The visually representative SVET current density maps and analysis displayed a different evolution and growth of anodic activity as inhibitor concentration was increased. The was due to the broader extent of anodic coverage at $1 \times 10^{-4} \mathrm{~mol} \mathrm{dm}^{-3}$, compared to the intermediate concentration. This can also be understood from the time-lapse microscopy data of Figure 5.7-5.8; the growth rate of anodic sites diminished as phosphate was 
introduced in greater amounts, resulting in the formation of precipitates on the surface. It is entirely possible that as corrosion developed, metal cations emergent from a site of anodic activity went on to react with phosphate anions present in solution. These metal phosphate precipitates seemed to readily form at sites of anodic activity where the concentration of metal ions was likely to accumulate in solution. This was focused within the eutectic regions, seemingly inhibiting these phases at early stages until corrosion progressed and the inhibitorbased precipitates deposited in these areas.

It is understood that different species of phosphate exhibit different solubility characteristics, thereby affecting the ability to deposit onto a surface and provide potentially advantageous properties in a corroding environment. Considering zinc phosphates, consisting of primary, secondary, and tertiary species, the solubility of these are recognised to decrease respectively, with the primary phosphate possessing the highest extent of solubility in water. Calculations from Table 5.4 and Table 5.5 indicated that the more beneficial tertiary species, comparative to the primary and secondary species, would be yielded in the experimental conditions via the solubility quotient exceeding the given solubility product. As discussed in 5.3.5, the insoluble nature of such zinc and magnesium phosphates would yield their precipitation proximal to these sites, as observed in Figure 5.7-5.8. Whilst the extent of this insoluble precipitation was not as marked in the time-lapse experiments for $1 \times 10^{-4} \mathrm{~mol} \mathrm{dm}^{-3}$, the polarisation data of Figure 5.10 indicated a reduction in $\log (\mathrm{I})$ to support this. This was also supported by the SVET data included in 5.3.1, and the Tafel values in Table 5.3. The overall suggestion from the data is that sodium phosphate acts as an anodic inhibitor for this system, forming proximal to sites of anodic activity and consequently restricting their growth through means of passivation.

The formation of the metal phosphate filaments in the time-lapse microscopy intervals of Figure 5.9 were of interest. These were observed to precipitate at sites of anodic activity but proceeded to deposit independently. Repeated experiments also demonstrated that in some cases, independent growth of the filament-like structures continued beyond the exposed sample surface, depositing onto the insulating PTFE adhesive tape. As illustrated in the Video 5.3 , this longitudinal growth was rapid. The combination of independent and rapid growth suggest that such filaments are consequent of a nucleation and growth mechanism initiated by a super saturation effect. It is entirely possible that an abundance of metallic ions in solution instigated the nucleation of a metal phosphate salt proximal to an anodic site. The continued growth of the precipitate may have ensued having already nucleated on the surface, where it grew independently of the underlying microstructural phases. This may explain how it was 
observed to precipitate away from the anodic site beyond the initial point of nucleation. Furthermore, as new filaments were observed to nucleate on existing ones, such events support this theory.

\subsection{Conclusions}

The SVET was used to study the corrosion behaviour of a ZMA alloy and the action of dosed sodium phosphate inhibitor additions in solution. The SVET data illustrated that for increasing concentrations of sodium phosphate inhibitor, the metal loss diminished, providing a large improvement in corrosion resistance levels. The corresponding current density maps revealed a progressively delayed anodic formation and diminished extent of anodic coverage, coincident with greater concentrations of inhibitor present. The time-lapse optical microscopy data established that progressive additions of sodium phosphate corresponded to a restriction in growth of anodic sites. This was via the formation of thin precipitates proximal to the anodic sites, specifically near eutectic regions at early stages of corrosion. At $1 \times 10^{-2} \mathrm{~mol} \mathrm{dm}^{-3} \mathrm{Na}_{3} \mathrm{PO}_{4}$, progression of the experiment led to the formation of a filament-like metal phosphate species. This is proposed to transpire through means of a nucleation and growth mechanism, having initiated from a super saturation of metallic ions in solution. The solubility calculations predicted that the conditions corresponding to all studied inhibitor concentrations enabled an insoluble tertiary phosphate species to precipitate on the surface. This corroborated the evidence gained by SEM-EDS, which indicated that the filaments deposited on the surface may have been of a magnesium phosphate composition. The polarisation data demonstrated a shift to more positive values of $E_{\text {corr }}$ accompanied by diminishing $\log (\mathrm{I})$ for increasing concentrations of sodium phosphate addition, matching the Evans diagram for anodic inhibition. The overall data strongly evidenced sodium phosphate acting to provide effective anodic inhibition. This is proposed to occur through reaction of metal cations emanant from an anodic site with that of phosphate anions, to form insoluble metal phosphate species. Despite the noted effectiveness of sodium phosphate as an inhibitor from the gathered data, it did not realise inhibition to the same degree as for the chromate compound reference point. 


\section{Chapter 6 Study of the inhibition effects of an amino acid during corrosion of a zinc-magnesium-aluminium (ZMA) galvanising alloy}

\subsection{Introduction}

This chapter considers the use of an amino acid, L-tryptophan, as an organic corrosion inhibitor addition within a ZMA system. In Chapter 5, the role of sodium phosphate as an inhibitor was investigated for the same ZMA alloy; the results strongly indicated that sodium phosphate acts as an anodic inhibitor under immersion conditions and that it can offer an appreciable degree of protection. A caveat exists in that it is understood to be harmful to aquatic life and consequently, legislation requires a restricted level of use [25]. A range of research has gone on to understand the effect of amino acids as corrosion inhibitors [29]-[32], [34]-[38]. These organic compounds are understood to be a non-toxic, biodegradable, relatively economical, and ultimately, environmentally-friendly class of inhibitor in comparison to those more traditionally used [104]. It has been suggested that in general, amino acids are difficult to specifically regard as either anodic or cathodic inhibitors, as this can change according to experimental conditions [105]. This results from changes in adsorption behaviour, however, it has been commonly reported that amino acids act as mixed type inhibitors.

Little work into the inhibitory effect of amino acids has been conducted for ZMA systems, and as such, that is the focus of this chapter. The sample material, as documented in previous chapters, is a steel substrate coated with zinc alloy containing small additions of magnesium and aluminium, the details of which are outlined in Table 6.1 below. The objectives of this work are to ascertain the effectiveness of L-tryptophan $\left(\mathrm{C}_{11} \mathrm{H}_{12} \mathrm{~N}_{2} \mathrm{O}_{2}\right)$ as a corrosion inhibitor for the stipulated galvanising alloy composition. The underlying inhibition mechanism of this individual amino acid was also investigated. Additions of L-tryptophan were introduced to the aqueous electrolyte at a given range of concentrations, and as such, corrosion behaviour was studied under immersion conditions.

Changes in localised surface corrosion events on the surface were mapped using the SVET, with varying concentrations of dosed inhibitor addition. Sample performance was compared using SVET-derived current density maps, area-averaged current density plots, and estimated metal loss values. This was in addition to open-circuit potential and potentiodynamic polarisation measurements to build on data obtained via SVET. Time-lapse optical microscopy was used to visualise corrosion in situ, on a microstructural level, to reveal the action of the inhibitor on the sample surface. Gravimetric analysis was performed as an indicator of accuracy 
for the SVET-based metal loss results. The experiments were conducted according to the different concentrations of L-tryptophan addition introduced.

\subsection{Experimental}

\subsubsection{Metallic specimens}

As outlined in Table 6.1 samples utilised for this chapter were a ZMA alloy coated steel, as provided by the French Corrosion Institute. These were manufactured under production line conditions.

Table 6.1 Composition of the zinc-magnesium-aluminium alloy

\begin{tabular}{|c|c|c|c|c|}
\hline Sample & Zn (wt\%) & $\mathrm{Mg}(w t \%)$ & $\mathrm{Al}(w t \%)$ & $\begin{array}{c}\text { Coating thickness } \\
(\mu \mathrm{m})\end{array}$ \\
\hline ZMA 2 & 96 & 2 & 2 & 10 \\
\hline
\end{tabular}

\subsubsection{Sample preparation}

As described in 2.1.2.1, ZMA 2 samples were cut using a guillotine into a $20 \mathrm{~mm} \times 20 \mathrm{~mm}$ profile and subsequently mounted. Mounted samples were metallographically polished to a $1 \mu \mathrm{m}$ finish, cleaned using distilled water and ethanol, etched, and once again cleaned. Identical masking procedures to that stipulated in Chapter 2 were completed, using adhesive PTFE tape to cover the sample surface and reveal $c a .100 \mathrm{~mm}^{2}$ and $0.785 \mathrm{~mm}^{2}$ areas exposed for both SVET and time-lapse microscopy experiments, respectively.

Preparations involving electrochemical testing in conjunction with a potentiostat followed a largely similar procedure, excluding the steps taken for PTFE masking. Instead, samples were removed carefully from their mounts, and securely placed into the customdesigned sample holder. Upon fitting, this exposed a sample area of $0.95 \mathrm{~mm}^{2}$. Details of this equipment can be found in 2.5-2.6.

\subsubsection{Experimental methodologies}

The SVET, electrochemical, and time-lapse microscopy experiments were all conducted by the submersion of samples in aqueous electrolyte. The electrolyte used throughout these experiments was $1 \% \mathrm{w} / \mathrm{v} \mathrm{NaCl}$ solution adjusted to $\mathrm{pH} 7$, as the control or baseline electrolyte free of inhibitor species. For both SVET and electrochemical testing procedures, additions of Ltryptophan (Trp) were added to this baseline solution ranging in concentration from $1 \times 10^{-4}$ up to $1 \times 10^{-2} \mathrm{~mol} \mathrm{dm}^{-3} \mathrm{Trp}$. The time-lapse microscopy experiments differed in that these were initiated in the baseline electrolyte (i.e. $1 \% \mathrm{w} / \mathrm{v} \mathrm{NaCl} \mathrm{pH} \mathrm{7)} \mathrm{beforehand.} \mathrm{Only} \mathrm{upon} \mathrm{formation} \mathrm{of}$ 
a distinctive anodic feature was the solution dosed with a designated concentration of inhibitor. Any $\mathrm{pH}$ adjustment to the electrolyte was made via droplet additions of dilute $\mathrm{NaOH}$ or $\mathrm{HCl}$ solution.

SVET experiment duration was 24 hours, active from the point of electrolyte introduction and being scanned each hour from this point. Setup, calibration, and data analysis associated with the SVET was performed as detailed in 2.2. A minimum of two repeat experiments for each inhibitor concentration was completed to contribute accuracy of the results. The electrochemical testing in the form of open-circuit potential (OCP) and polarisation tests were recorded versus a saturated calomel electrode (SCE). Across the range of inhibitor concentrations considered in this work, polarisation tests were swept at a range of $-1.4 \mathrm{~V}$ up to $0 \mathrm{~V}$, with a sweep rate of $1 \mathrm{mV} \mathrm{s}^{-1}$. The methods utilised in these procedures are stated in further detail in 2.5-2.6. Gravimetric analysis was performed to clarify the accuracy of SVETderived results, the procedures of which are set out in detail in 2.7. This involved testing two concentrations of inhibitor over a 24-hour period and subjecting the samples to multiple chemical cleaning cycles using a saturated glycine solution within an ultrasonic bath. This was performed until the corrosion products were removed from the surface.

\subsection{Results and discussion}

\subsubsection{Determination of corrosion inhibition of a ZMA alloy by L-tryptophan using SVET}

The corrosion inhibition effects of Trp and the associated mechanisms on ZMA coated steel samples (see Table 6.1) were studied. This was conducted in a baseline electrolyte of $1 \% \mathrm{w} / \mathrm{v}$ $\mathrm{NaCl}$ neutralised to $\mathrm{pH} 7$, with experiments including $1 \times 10^{-4}$ up to $1 \times 10^{-2}$ L-tryptophan mol $\mathrm{dm}$ ${ }^{3}$ additions. The freely corroding surfaces of ZMA samples were mapped by SVET, resolved as a distribution of the normal current density at the given scan intervals. The maps depicted in Figure 6.2-6.4 extend from hour 1, the point at which the scans were initiated, to the final scan at hour 24. The results for the uninhibited ZMA 2 sample (1\% w/v NaCl pH 7) from Chapter 3 (Figure 3.11) have been utilised as a point of reference for the performance relative to the inhibited sample conditions. The data extracted from SVET was used to produce a semiquantitative value of metal loss for the range of concentrations tested, given in Figure 6.1. The metal loss data displayed an immediate inverse relationship between concentration of Trp and level of metal loss measured during the testing procedure. The smallest addition to the number of $1 \times 10^{-4} \mathrm{~mol} \mathrm{dm}^{-3}$ exhibited a near $20 \%$ decrease in measured metal loss comparative to the control. This was followed by a decrease to approximately $63 \%$ of the measured value at $1 \times 10$

${ }^{3}$ L-tryptophan $\mathrm{mol} \mathrm{dm}^{-3}$, relative to the uninhibited sample. A very much significant reduction in 
the metal loss value was then observed for the highest inhibitor concentration $\left(1 \times 10^{-2} \mathrm{~mol} \mathrm{dm}^{-}\right.$ ${ }^{3} \operatorname{Trp}$ ) to less than $3 \%$ of the original, uninhibited sample. The results immediately highlighted that the presence of Trp was effective at altering the corrosion performance of the ZMA alloy in question, and to a substantial extent for the greatest concentration.

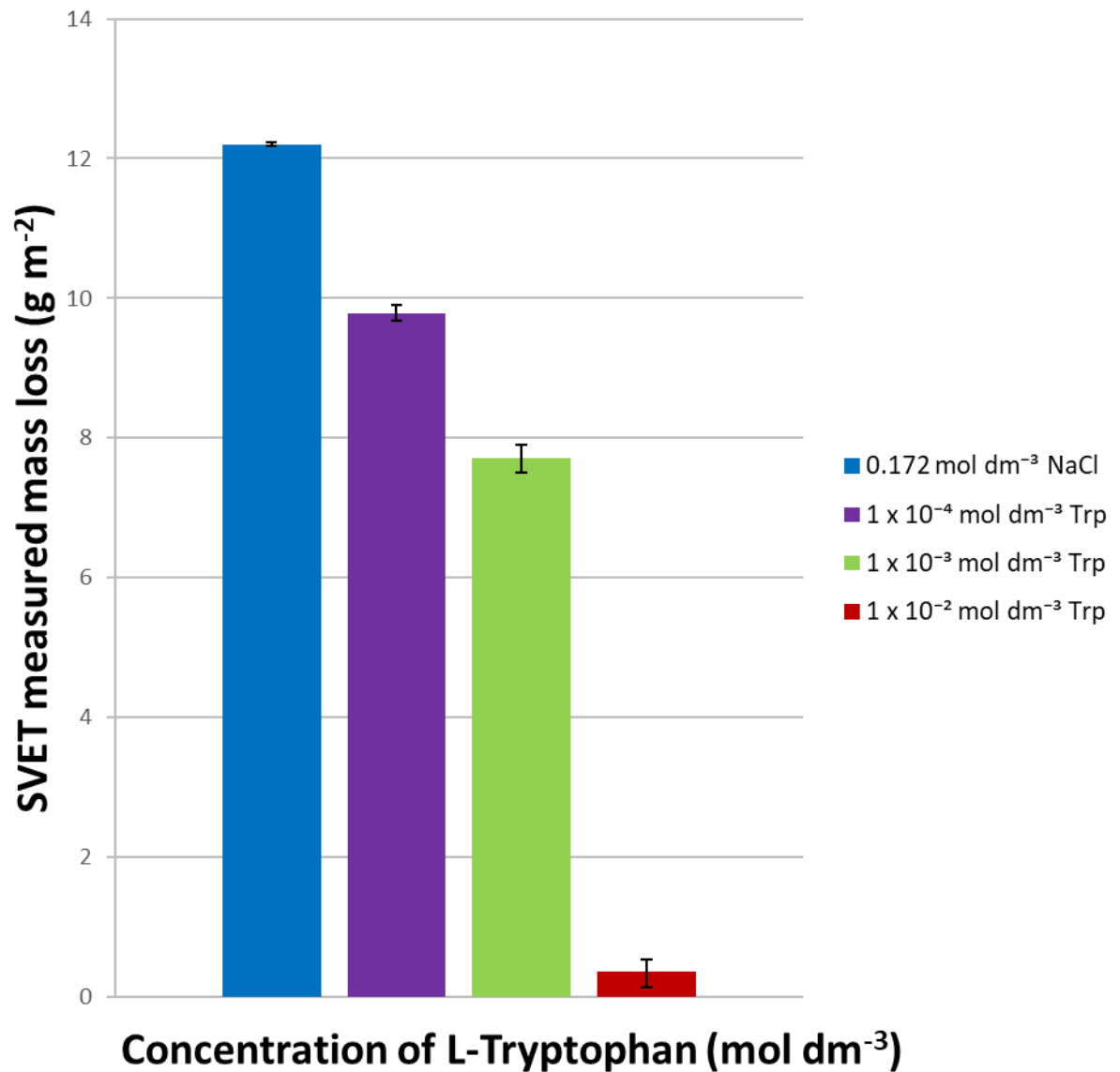

Figure 6.1 SVET estimated metal loss for ZMA 2 over 24-hour experiment time in $1 \% \mathrm{NaCl}$ pH 7 with given concentrations of L-tryptophan inhibitor

The surface corrosion activity captured by SVET for the $1 \times 10^{-4} \mathrm{~mol} \mathrm{dm}^{-3}$ addition is given in Figure 6.2. The map at hour 1 evidenced a range of anodic sites distributed on the surface, most of which were of point anode profile, with some indication of coalescence at the bottom-right hand corner of the sample. The intensity of some of these anodes was seen to fade, whilst other sites were newly formed and again of point anode profile by hour 4 . Hour 8 displayed the continued formation of anodic activity and evidenced the transition of these point sites into coalesced 'islands'. Whilst anodic activity was sustained overall between hours $12-24$, the majority of this encompassed the top half of the sample. During this time, the anodic islands were seen to grow, many of which exhibited an outward radial progression, and were of mild intensity overall. This covered a large proportion of the available surface by hour 24 . Figure 6.3 displayed that of the increased Trp concentration by an order of magnitude; a multitude of 
point anodic sites were present at hour 1 which varied in size, but were smaller than those observed in the Figure 6.2 counterpart. Upon hour 4, the larger anodes possessed diminished intensity, whilst the smaller anodes had experienced clear growth and retained their individual point profile. As the experiment continued, a gradual broadening of the anodic sites was present, in tandem with the coalescence of these sites. The radial, outward progression of existing anodes became apparent at hour 16 , and this continued in a steady fashion until the close of experiment, where the intensity of such sites was wholly reduced. By the final scan at hour 24, a large proportion of the surface was occupied by anodic activity. The current density maps for the highest Trp addition at $1 \times 10^{-2} \mathrm{~mol} \mathrm{dm}^{-3}$ given in Figure 6.4 , was highly divergent relative to the other inhibitor concentrations considered. At hour 1, a small amount of very mild anodic activity was observed. However, very little anodic activity was visible on the surface subsequent to this scan.

There was fitting agreement between data sets from the plot of metal loss results and the current density distribution maps, which displayed appropriate degrees of effectiveness according to inhibitor concentration. Alone, these results exhibited a great extent of effectiveness of corrosion inhibition for the highest concentration of Trp when introduced into solution. The effectiveness of this addition was also clear for the lesser concentrations, but in a less pronounced manner. The anodic growth plots for each inhibitor concentration, as plotted in Figure 6.5, sufficiently supported these trends. The highest Trp concentration displayed no growth beyond hour 1, concurrent with the largely improved values of metal loss. The remaining concentrations offered similar rates of growth to one another, with the intermediate concentration supporting a generally smaller size of anodic feature. This coincided with a lessened extent of anodic coverage and intensity than observed for the lowest concentration. 

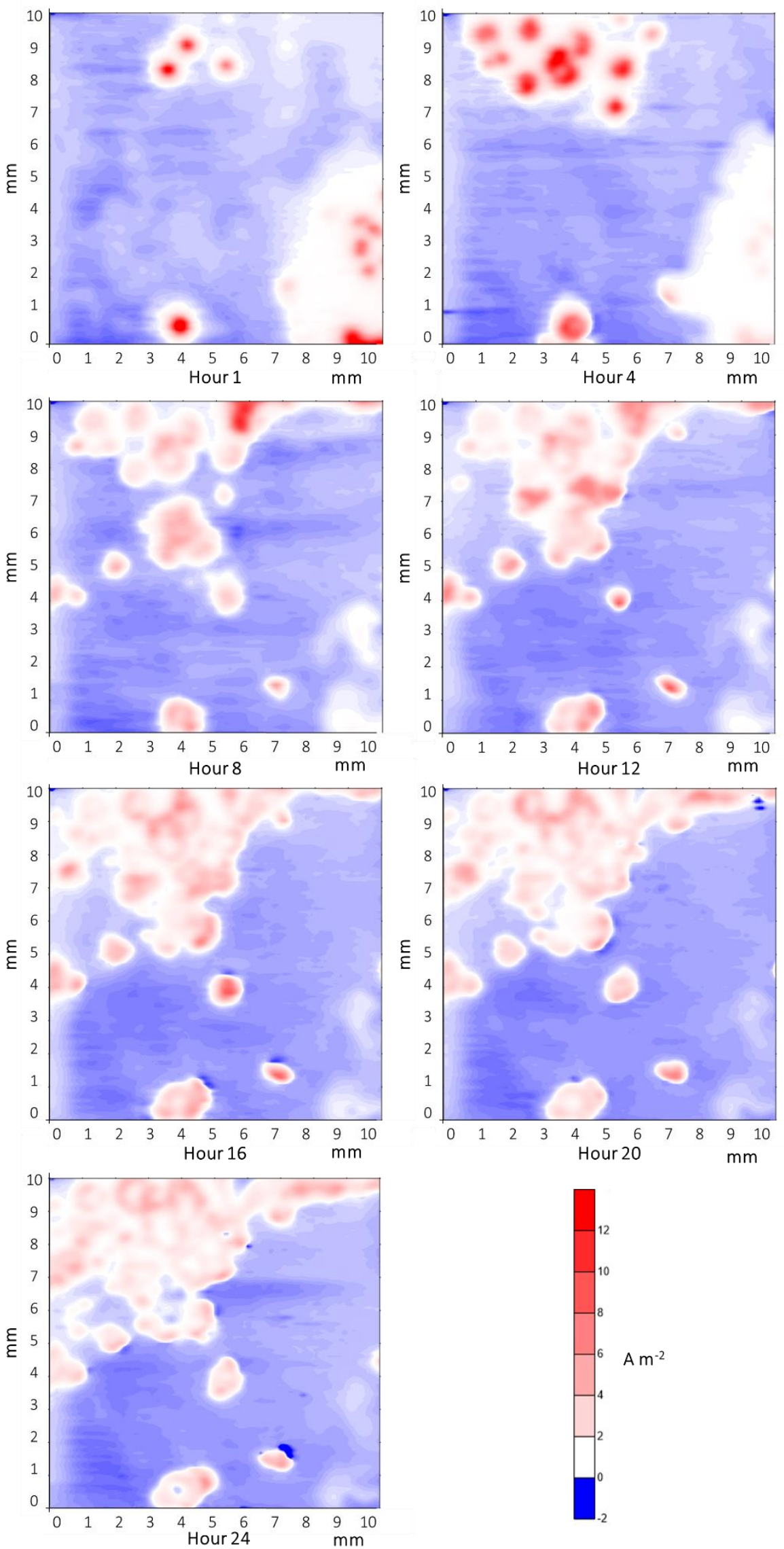

Figure 6.2 SVET normal current density maps of ZMA 2 in $1 \% \mathrm{NaCl} \mathrm{pH} \mathrm{7,} \mathrm{including} 1 \times 10^{-4} \mathrm{~mol}$ $\mathrm{dm}^{-3}$ L-tryptophan 

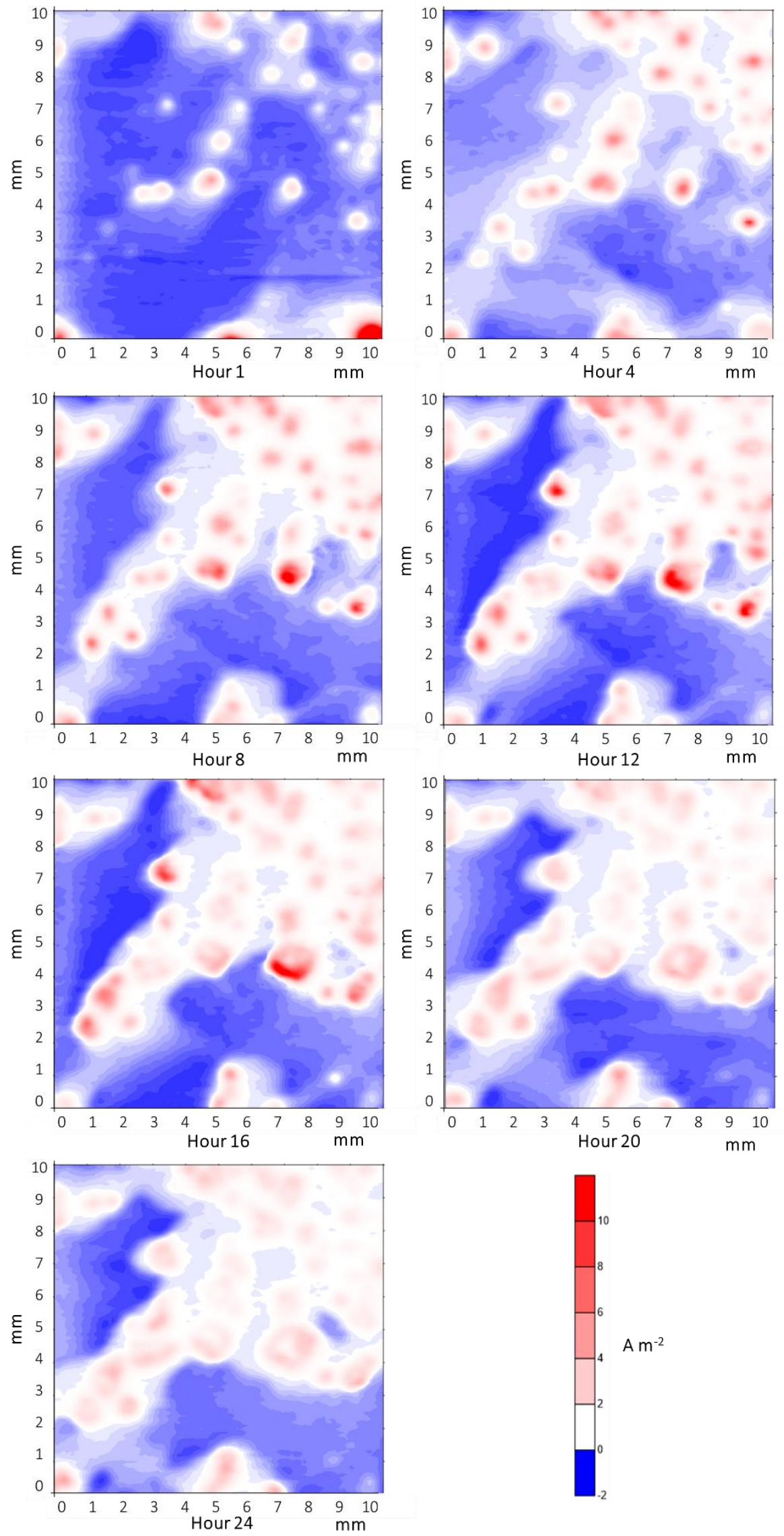

Figure 6.3 SVET normal current density maps of ZMA 2 in $1 \% \mathrm{NaCl} \mathrm{pH} \mathrm{7,} \mathrm{including} 1 \times 10^{-3} \mathrm{~mol}$ $\mathrm{dm}^{-3}$ L-tryptophan 

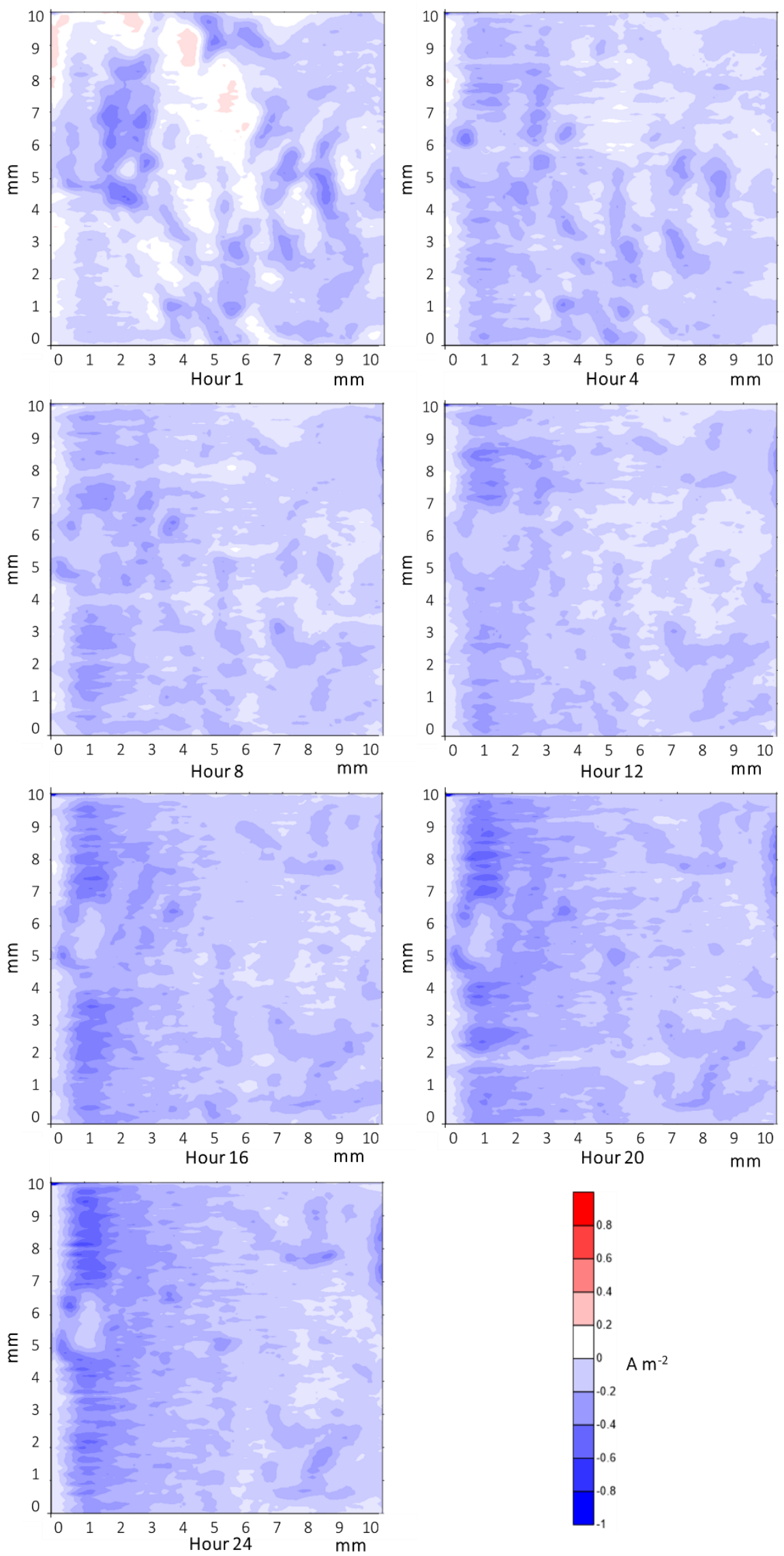

Figure 6.4 SVET normal current density maps of ZMA 2 in $1 \% \mathrm{NaCl} \mathrm{pH} \mathrm{7,} \mathrm{including} 1 \times 10^{-2} \mathrm{~mol}$ $\mathrm{dm}^{-3}$ L-tryptophan 
In Figure 6.6, the progress of anodic activity over selected SVET experiments is given via the area-averaged anodic current density $\left(\mathrm{Ja}_{\mathrm{t}}\right)$ values plotted as a function of time. The inhibited control sample was included as a point of reference for the remaining parameters. The $1 \times 10^{-4}$ mol dm ${ }^{-3} \operatorname{Trp}$ addition did not undergo large variations compared to the control sample and retained the general trend of the plot, maintaining a proportionally lower value of $\mathrm{Ja}_{\mathrm{t}}$ throughout; this can be understood as the effect of the inhibitor lowering the current associated with corrosion, thereby lowering the value registered by SVET. At $1 \times 10^{-3} \mathrm{Trp}$ mol $\mathrm{dm}^{-3}$, an overall fall in $\mathrm{Ja}_{\mathrm{t}}$ was seen to continue accompanied by change in anodic progression. Unlike that of the control sample and smallest inhibitor concentration, the onset of activity was diminished for several hours, before rising sharply and tapering off around hour 10 . This activity was measured to fall sharply at hour 16 , coincident with the decline in anodic intensity observed in Figure 6.3, before stabilising at hour 20 until the end of the experiment. As displayed by the corresponding SVET current density maps, the maximum utilised concentration differed greatly to the remaining plots. Initial Jat measurements were the largest observed, but this declined sharply until hour 2 . From this point, the values were realised to be minimal throughout the remaining length of experiment, fully corroborating the SVET-produced maps. As observed from the SVET-derived, there was a clear difference in inhibition performance for the three different concentrations employed. The highest concentration of inhibitor offered a good degree of effectiveness comparatively, however, the intermediate concentration exhibited signs of diminishing Jat value in the latter hours. Whilst by no means definitive, this may suggest that the inhibitory effects offered at this intermediate concentration $\left(1 \times 10^{-3} \mathrm{Trp}\right.$ mol dm${ }^{-3}$ ) extend beyond the SVET experiment time-frame of 24 hours. 


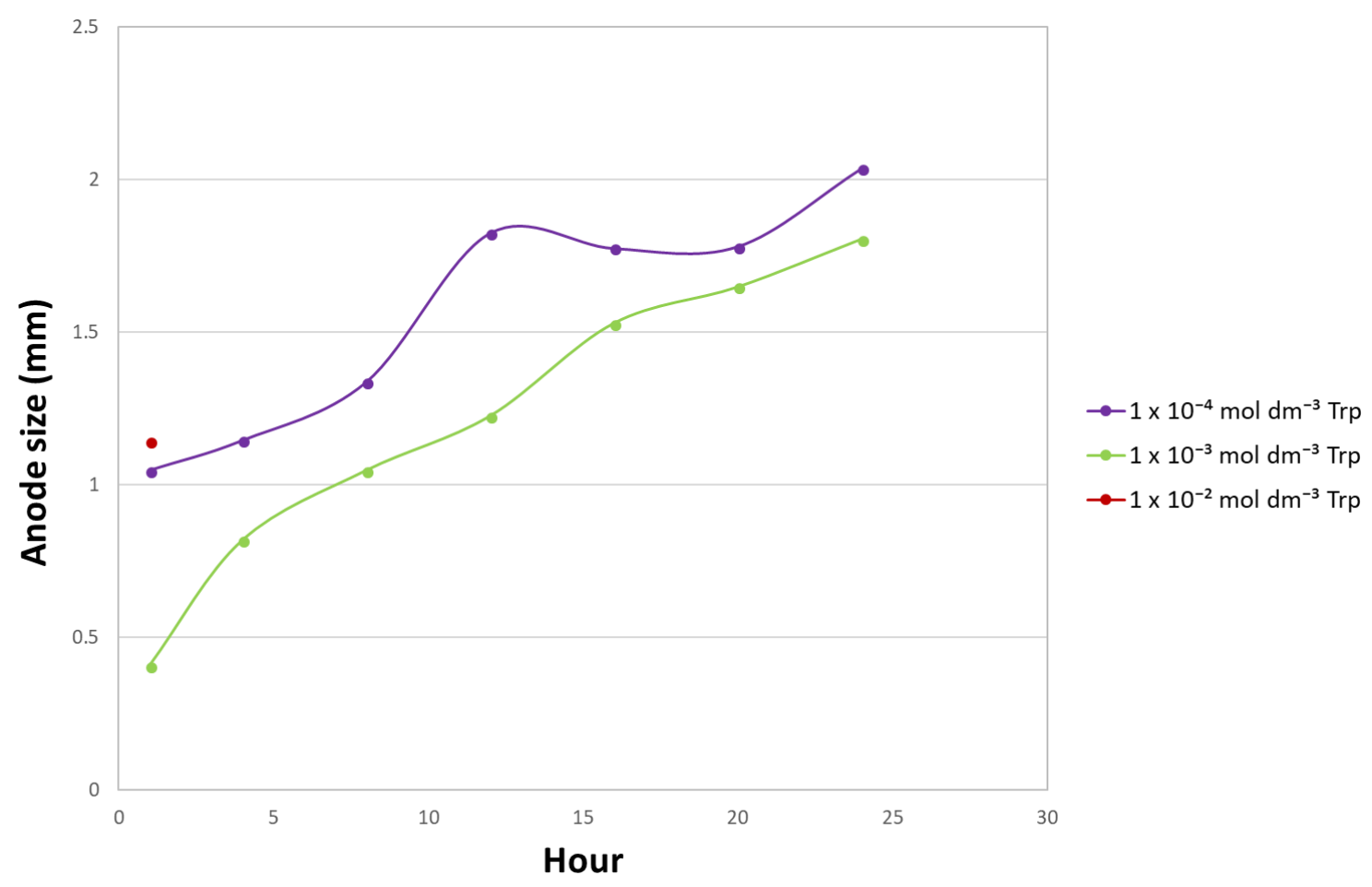

Figure 6.5 Plot of SVET anode size as a function of time for L-tryptophan

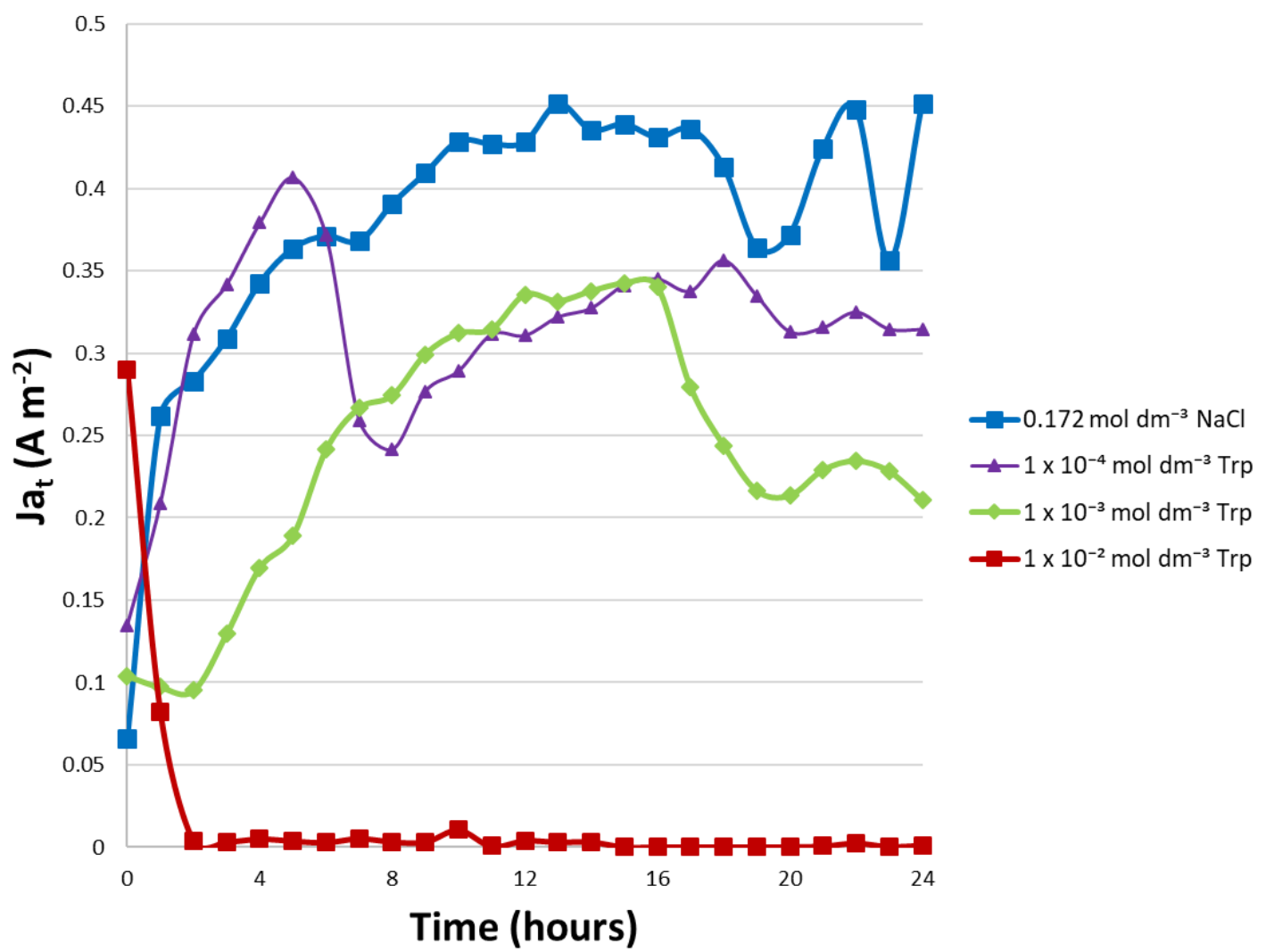

Figure 6.6 The area-averaged anodic current density $(\mathrm{Jat})$ plotted as a function of time for ZMA alloy in $1 \% \mathrm{NaCl} \mathrm{pH} 7$ electrolyte, encompassing the range of L-tryptophan inhibitor additions 


\subsubsection{Time-lapse optical microscopy examination of L-tryptophan interaction as a}

corrosion inhibitor on a ZMA alloy

Time-lapse optical microscopy was utilised to observe the effect and action of L-tryptophan on the ZMA 2 alloy in question. The associated digital images were captured at designated twominute intervals over a minimum period of 20 hours. The aqueous electrolyte was dosed with the inhibitor at the described range of concentrations, following the formation of an established anodic site. The arrangement of Figure 6.7 E-P constitute an initial image from the experiment onset, an image taken immediately prior to the inhibitor introduction, followed by two images captured in 60-minute successive intervals. Figure 6.7 A-D differ in that these images correspond to 60-minute intervals only, taken from Chapter 3 experiments; these are included as a point of reference, as an uninhibited counterpart. Further to the included time-lapse images, the full set of images captured during an experiment were collated into videos according to each of the inhibitor concentrations used. The uninhibited, control sample is given in Video 3.2, whereas Videos 6.1-6.3 are included in order of increasing inhibitor concentration (i.e. Video 6.1 corresponds to the $1 \times 10^{-4} \mathrm{~mol} \mathrm{dm}^{-3}$ addition).

Figure 6.7 E-H represent a time-lapse experiment for the lowest concentration of Trp in this chapter. Image E displayed little visible activity on the surface, but contrasted the prominent anode that was present in the following image $F$. In the latter image, the darker area was the region of focused anodic attack, with no other distinct anodes perceivable elsewhere on the surface. A darkened area was also noticeable at a certain proximity from the anodic site, forming what may be realised as an arc of corrosion product precipitated towards the centre of the sample. This provided good indication of the boundary between anodic and cathodic sites, with the intermediate corrosion product acting as a border [79]. Upon image $\mathrm{G}$, the original anode continued as the focal site, which was seen to grow between these intervals. The attack was visibly preferential in the eutectic regions on this scale, leaving regions of the primary dendrites intact. The corrosion product precipitate progressed via increased darkening, indicating its continued deposition; this also appeared to extend, spreading further away from the focal anode. The growth of the focal anode and enrichment of the corrosion product continued into image $\mathrm{H}$. The anodic attack was sustained, exhibiting attack extending to the local primary dendrites. The layers of corrosion product continued to accumulate and expand, depositing on more of the surface simultaneously, reaching the periphery of the exposed surface. As the inhibitor addition was administered immediately after the image $F$ interval, the surface deposition observed between images G-H may not have exclusively constituted corrosion product conventional to the ZMA 2 alloy; instead, this may have formed in tandem 
with a product yielded by the inhibitor addition. The progression of surface activity was not dissimilar to that of Figure 6.7 A-D of the control sample, indicating that no significant effects transpired within this time-frame as a result of the inhibitor addition.

Figure 6.7 I-L considered Trp additions at $1 \times 10^{-3} \mathrm{~mol} \mathrm{dm}^{-3}$. Comparison of images I-J indicated the onset and growth of an anodic site as the most distinct change, with little other difference visible between these image intervals. The sustained growth of the focal anode was clear upon image $\mathrm{K}$, with the concurrent precipitation of a surface film at a given distance from said activity. The surface film was of a discontinuous ring profile, showing a decreased compactness or density with distance from the initial point of formation. Image $L$ again clarified the continued growth of the anode, but in a more moderate manner than the previously discussed concentration. The enrichment of the surface film was evident, with its progression extending into the cathodic region, exhibiting the formation of clusters of small precipitates. This surface layer appeared to be more continuous relative to the lowest inhibitor addition.

The time-lapse images associated with the highest inhibitor concentration $\left(1 \times 10^{-2} \mathrm{~mol}\right.$ $\mathrm{dm}^{-3} \mathrm{Trp}$ ) are given in Figure 6.7 M-P. Contrasting images M-N, the anodic formation was clear at image $\mathrm{N}$ towards the bottom of the exposed surface. Once again, a corrosion product was also visible, depositing at a distance away from the distinct anodic feature. Upon image $\mathrm{O}$, a significant difference was present on the sample surface. Beyond the corrosion product, and in the regions of cathodic activity, was a translucent and near-continuous film precipitated onto the surface. This deposit also appeared to extend beyond the corrosion product boundary (towards the anode) in a less marked fashion. It was also the case that minimal growth of the focal anodic feature was observed, coincident with the deposition of the surface film. The ability to discern the underlying microstructure indicated that the film was likely to be thin. Image P exhibited an increasingly darkened appearance of the film, probably due to increased deposition of the layer. This was the case for the majority of the surface, including at a distance more proximal to the anode. The anode underwent minimal growth perceivable from the timelapse image, coincident with the formation of the encompassing surface film. Understanding that the Trp addition was introduced following image $\mathrm{N}$, it can be realised that the surface layers present in the latter images were a result, indicating that this was a product of the inhibitor. As indicated more clearly in Video 6.3, upon introduction of the inhibitor into solution, a portion of the existing corrosion product associated with the alloy was seen to resolubilise. This effect may have been due to $\mathrm{pH}$ changes consequent of the inhibitor dosing, allowing the insoluble species to become soluble in solution once more. As the time-lapse sequence continued beyond the intervals outlined in Figure 6.7, the coverage of the surface film 
was observed to extend beyond the cathodic area and was noticed to form around the anodic region also.

As observed, the extent of the deposition associated with the highest concentration of inhibitor was strikingly greater than observed for the other concentrations. This also corresponded to the severe restriction in growth of the anodic site, which was less so the case for the intermediate concentration. As a distinct layer formed following the introduction of the Trp, it can be realised that this was a result of the inhibitor interacting with the surface in some manner. As the concentration of inhibitor increased, the extent of surface film coverage followed, in tandem with a reduced extent of anodic growth. This provided evidence that the inhibitor acted to restrict anodic growth via a surface layer, depositing primarily in areas of cathodic activity. As the anodic attack visibly began in the eutectic phases, the surface layers were not seemingly present in these regions, instead forming at those phases supporting cathodic activity. Consequently, it can be understood that under these conditions, the Trp addition appeared to act as a cathodic inhibitor; this was via deposition within cathodic regions, accumulating over time. This would act as a barrier to oxygen adsorption at the surface, limiting the cathodic oxygen reduction reaction and ultimately, restricting the kinetics of the overall corrosion process.

Additional data was extracted from the time-lapse images to produce a plot of corroded area as a function of time, as given in Figure 6.8. This was based upon measurements of the anodic area. This set of measurements assumed that corrosive attack progressed in a lateral manner across the surface, and that vertical pitting did not occur in excess, due to the limited two-dimensional view of the technique. This analysis was performed using Photoshop CS6 software, whereby the area of anodic attack was selected and measured. The measurements were calibrated on a pixel-distance ratio via an image of identical resolution and magnification. Image intervals for each hour were analysed up to a limit of 20 hours, as beyond this point microstructural features were obfuscated and could not be sufficiently resolved. The control data for the uninhibited sample was also included for its experimental time-frame as a reference indicator. The plot of Figure 6.8 illustrated the differences in anodic progression for the three different concentrations of inhibitor. The plot highlighted that there were clear changes in the rate of anodic growth, with an inverse relationship between concentration of inhibitor and anodic growth rate present. The plot corresponding to $1 \times 10^{-4} \mathrm{~mol} \mathrm{dm}^{-3} \operatorname{Trp}$ revealed a linear growth rate until hour 6 , where this continued at a diminished rate until hour 9; at this point the steeper gradient was observed and present for the remainder of the plot. The control counterpart was generally linear also, but showed an overall reduced extent of 
anodic attack comparative to the lowest inhibitor concentration. The intermediate concentration offered a shallower gradient, understood from the slower anodic growth observable in Figure 6.7. A similar effect was seen for the highest concentration of inhibitor, yet to a much greater degree, and again displaying a relatively linear plot. The plot in its entirety graphically represents the extent of the growth observed during the time-lapse sequences, which highlighted the effect of the inhibitor on the anodic growth process during a corrosion reaction.

The time-lapse data was further manipulated to obtain an overall view of the corroded area for the 20-hour period analysed, as represented in Figure 6.9. As the control experiment was of a shorter time-frame than the inhibited counterparts, an estimated value of total corroded area was included via linear extrapolation of the data presented in Figure 6.8. The plot revealed a maintained inverse relationship between inhibitor concentration and extent of corroded area, as seen in the SVET-derived metal loss values (Figure 6.1). The findings from this total corroded area plot and the SVET metal loss values indicated a deviation of less than $1 \%$ in the instance of the highest inhibitor concentration, where the $1 \times 10^{-2} \mathrm{~mol} \mathrm{dm}^{-3} \operatorname{Trp}$ exhibited a reduction in excess of $97 \%$ and $96 \%$ respectively, relative to the $1 \times 10^{-4} \mathrm{~mol} \mathrm{dm}^{-3}$ addition. The resemblance between data sets was not noticed for the intermediate concentration however, where the time-lapse plot measured a proportionally smaller value than that measured via SVET. A reason for this may have been the two-dimensional restriction of the technique itself, whereby the time-lapse scrutinises the data visually, and the SVET instead realises the emanation of ionic current flux. The results indicated that the $1 \times 10^{-4} \mathrm{~mol} \mathrm{dm}^{-3} \mathrm{Trp}$ addition brought about a diminished performance relative to the control sample estimated value. However, the intermediate concentration and beyond indicated that the inhibitor was effective via means of restricted anodic growth. 

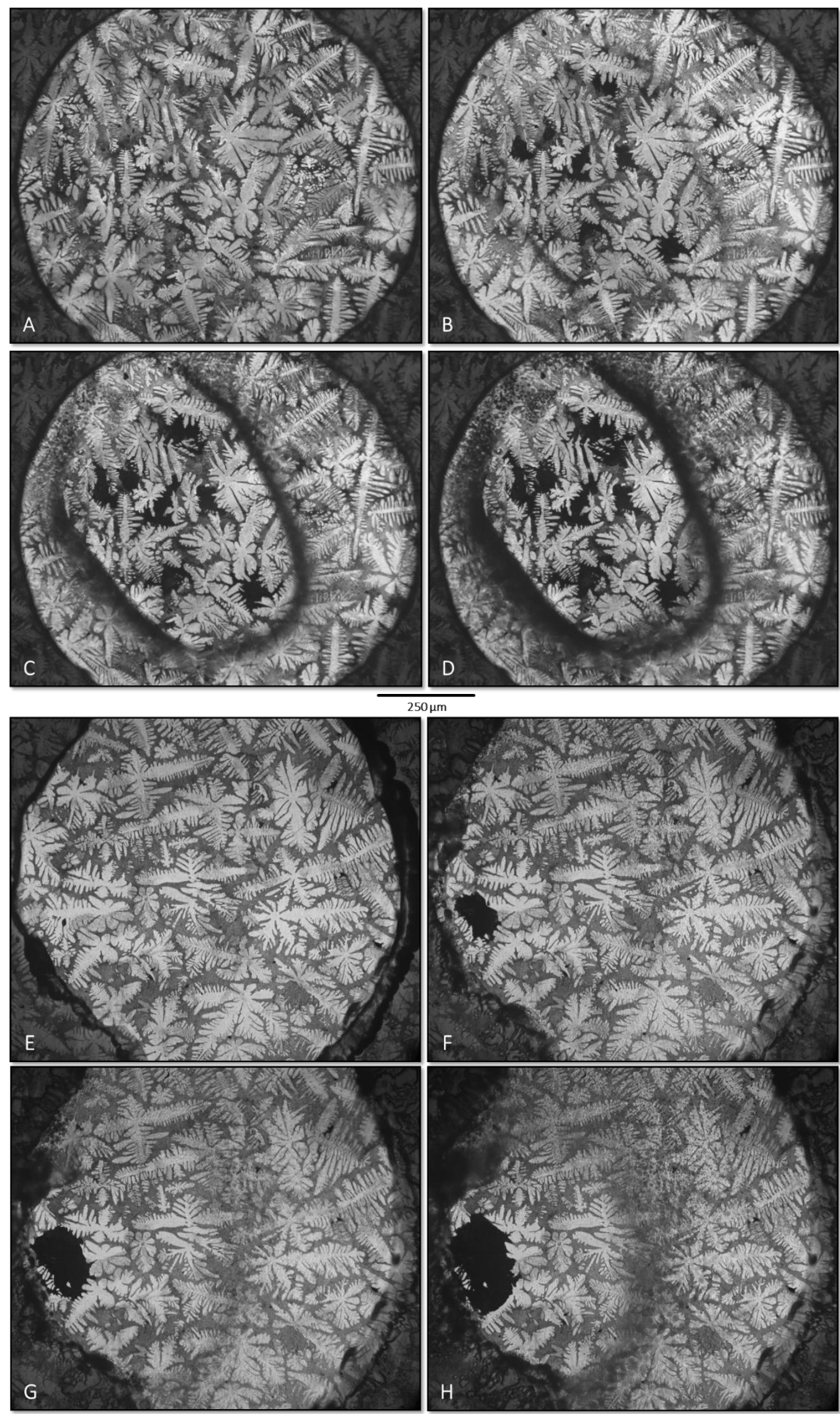

Figure 6.7 Time-lapse microscopy images taken at hourly intervals in a baseline $1 \% \mathrm{NaCl}$ electrolyte, including: (A-D) uninhibited control, (E-H) $1 \times 10^{-4} \mathrm{~mol} \mathrm{dm}^{-3} \operatorname{Trp},(\mathrm{I}-\mathrm{L}) 1 \times 10^{-3} \mathrm{~mol} \mathrm{dm}^{-3}$ Trp, (M-P) $1 \times 10^{-2} \mathrm{~mol} \mathrm{dm}^{-3} \operatorname{Trp}$ 

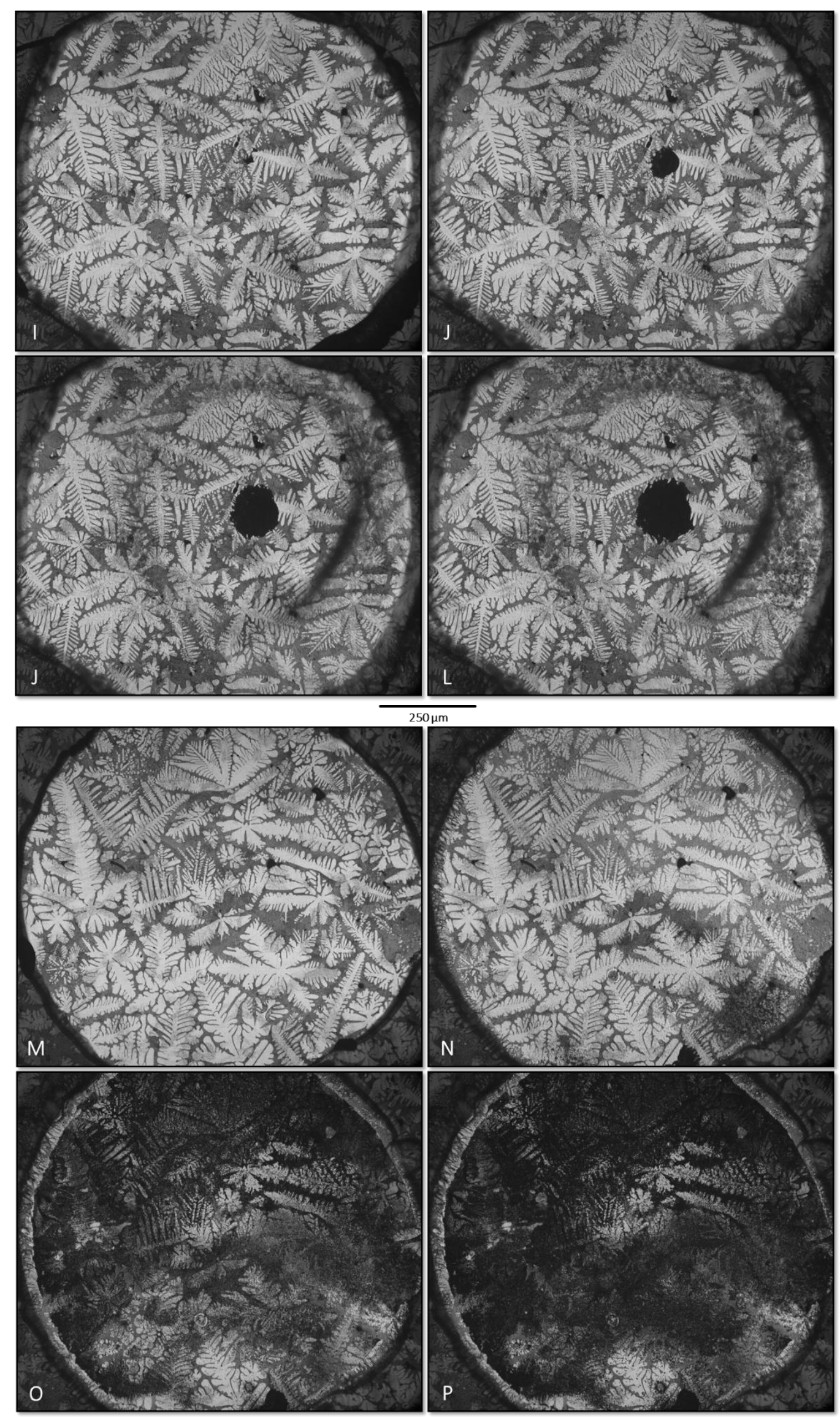

Figure 6.7 Continued 


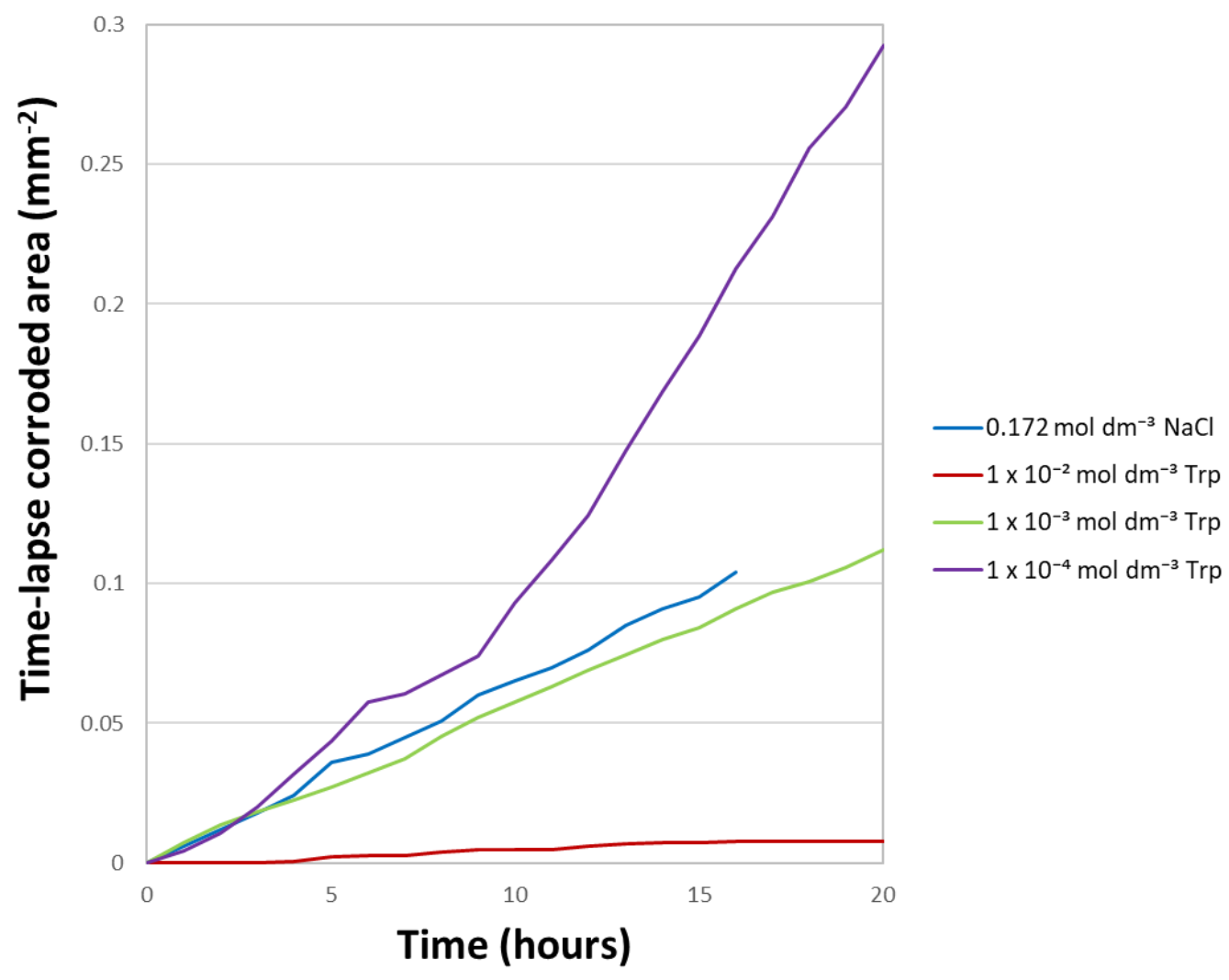

Figure 6.8 Size of corroded area versus time for each inhibitor concentration in $1 \% \mathrm{NaCl}$ electrolyte

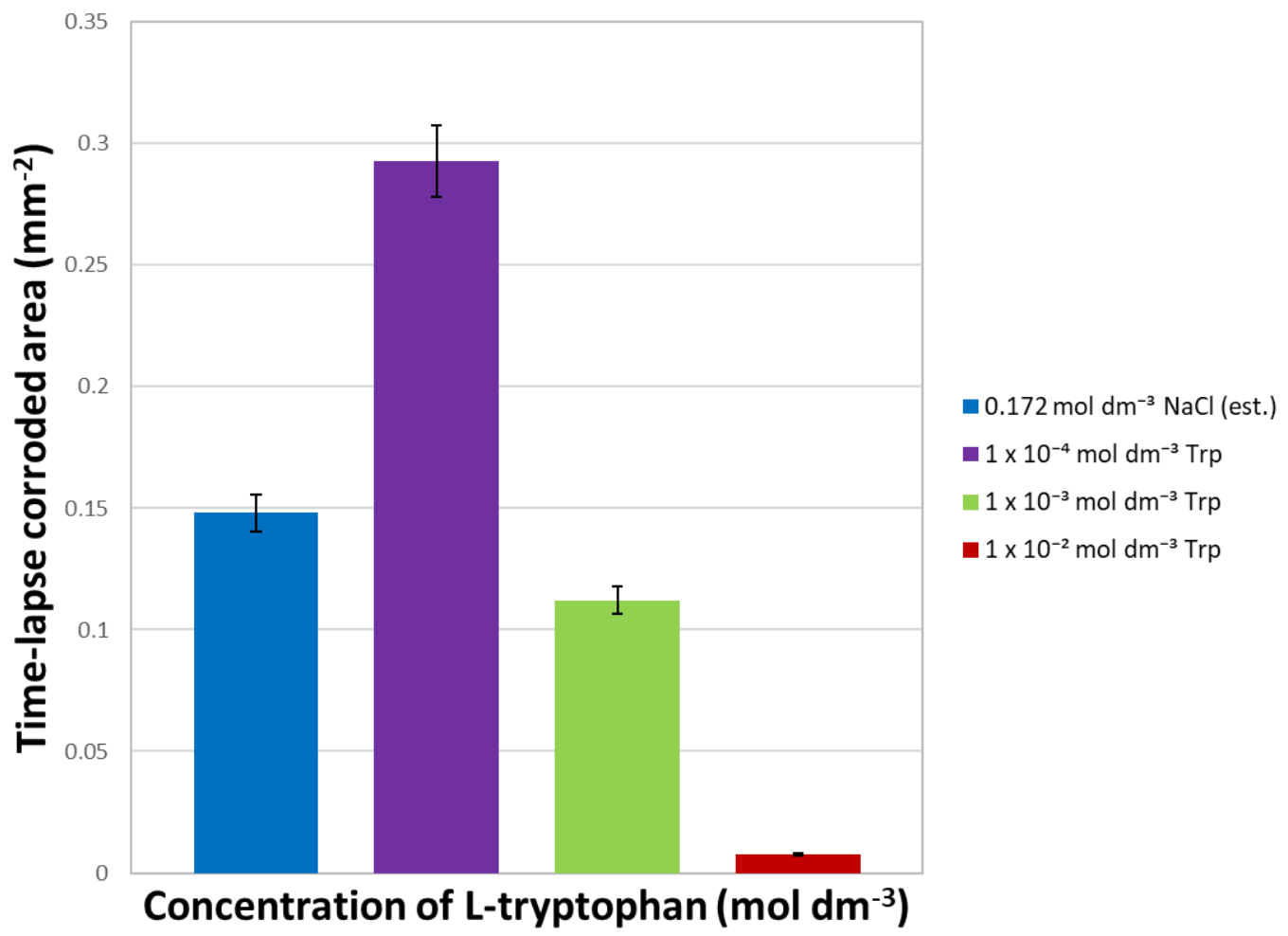

Figure 6.9 Total corroded area for each inhibitor concentration in $1 \% \mathrm{NaCl}$ electrolyte 
6.3.3 Open-circuit potential and potentiodynamic polarisation of varying concentrations of L-tryptophan

Further experimentation was performed to elaborate on the mechanisms of action for Ltryptophan within the ZMA system. This was achieved via electrochemical testing in the form of open-circuit potential and potentiodynamic polarisation work, in conjunction with a potentiostat. For open-circuit potential testing, samples were immersed in solution for 30 minutes where they were free to corrode. The electrolyte considered for this work was a baseline $1 \% \mathrm{w} / \mathrm{v} \mathrm{NaCl} \mathrm{pH} 7$ solution free of inhibitor, and the range of Trp inhibitor additions were tested from $1 \times 10^{-4} \mathrm{~mol} \mathrm{dm}^{-3}$ up to $1 \times 10^{-2} \mathrm{~mol} \mathrm{dm}^{-3}$. This same range of electrolyte was utilised for the polarisation work. Whilst polarisation testing typically involves sweeping cathodically and anodically (respective to the open-circuit measurement) in a single run, the results included for this work were performed differently. To study the impact of the Trp additions on sample corrosion behaviour, the tests involved instigating individual sweeping protocols relative to their open-circuit. The sample was immersed into solution and left to corrode at its open-circuit for 30 minutes, to reach a stable value. The sample was polarised marginally positive of the open-circuit and was swept in a cathodic-going fashion from -1 $\mathrm{V}$ to 1.5 $\mathrm{V}$ vs SCE. In a similar fashion for the anodic sweep, a further sample was left to corrode at its open-circuit for 30 minutes. The sample was subsequently polarised somewhat negative to its open-circuit value, and then swept towards $0 \mathrm{~V}$ vs SCE. The individual polarisation tests were conducted for each parameter to determine the impact of the inhibitor additions on the system, rather than in a consolidated fashion that may distort the interpretation of the results. 


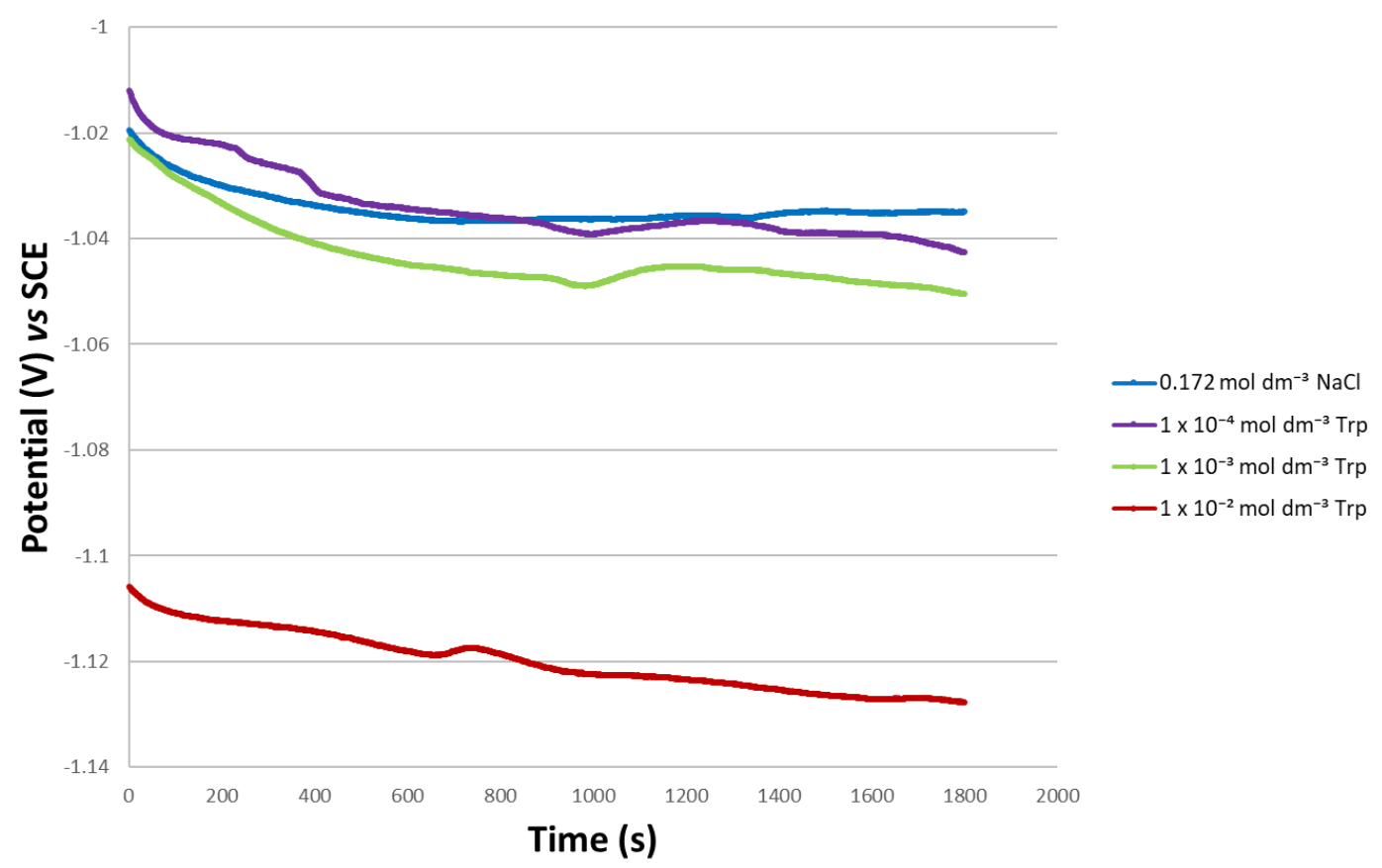

Figure 6.10 Plot of open-circuit potential testing (versus SCE) over the range of dosed inhibitor concentrations in $1 \% \mathrm{NaCl} \mathrm{pH} 7$ baseline electrolyte

The open-circuit potentials measured, as displayed in Figure 6.10, were all observed to fall from their initial measurement. After 30 minutes, significant differences in potential were observed. The results provided a definite trend, with the uninhibited, control sample offering the highest $E_{\text {corr }}$ of $-1.035 \mathrm{~V}$ (vs SCE). A progressive decrease in the $E_{\text {corr }}$ value was coincident following the increasing concentrations of Trp additions into solution, extending to $-1.128 \mathrm{~V} v \mathrm{~S}$ SCE for the $1 \mathrm{x}$ $10^{-2} \mathrm{~mol} \mathrm{dm}^{-3}$ addition. This exhibited an inverse relationship between $\mathrm{E}_{\text {corr }}$ and the inhibitor concentration, highlighting that any increase in Trp addition correlated with a measured fall in $E_{\text {corr. }}$ The Evans diagram for cathodic protection (see Figure 1.9) demonstrates that a cathodic inhibitor acts to reduce the value of $i_{\text {corr }}$ concurrently with $E_{\text {corr }}$. The diminishing $E_{\text {corr }}$ values from this plot link well with such a mechanism, which is highly suggestive that the Trp additions acted in this manner. This was especially the case for the highest inhibitor concentration, which revealed a much larger decrease in $\mathrm{E}_{\text {corr }}$ than the other tested parameters. This was further highlighted by the associated improvements in corrosion performance from both SVET and time-lapse data sets. The combination of evidence strongly suggested that under these circumstances, Trp acted primarily as a cathodic inhibitor.

The polarisation curves relating to the cathodic-going sweep are given in Figure 6.11. The value of $E_{\text {corr }}$ for the $1 \times 10^{-2} \mathrm{~mol} \mathrm{dm}^{-3}$ Trp once again displayed a shift to a more negative potential comparative to the control sample, with little change visible for the lower inhibitor concentrations. Considering the set of curves, a steep rise into cathodic currents (log (I)) existed 
from the point of open-circuit. Beyond this initial gradient, a plateau was present, indicating a point of limiting diffusion kinetics; this is where the cathodic reaction is governed by the rate of oxygen diffusion towards the metal-electrolyte interface. The small peaks at approximately -1.2 V (vs SCE) for the control sample and lowest inhibitor addition could indicate the formation of a surface oxide or hydroxide, although this would require clarification. A subtle peak was also noted to a much smaller extent in the $1 \times 10^{-3} \mathrm{~mol} \mathrm{dm}^{-3}$ addition. Interestingly, this peak was not visible at the highest inhibitor concentration, suggesting that a more developed surface layer adsorbed onto the metal was present, thereby hindering formation of the oxide/hydroxide. Another set of peaks were observable at more negative potentials, such as that present from ca. $1.35 \mathrm{~V}$ (vs SCE) for $1 \times 10^{-4} \mathrm{~mol} \mathrm{dm}^{-3} \mathrm{Trp}$. Once again, these peaks may correspond to the reduction of an oxide/hydroxide formation, as this was not perceivable in the curve for the highest concentration of Trp. The values of $\log (\mathrm{I})$ were observed to return to similar levels previously discerned as $E_{\text {corr }}$ values were swept towards the final value of $-1.5 \mathrm{~V}$ vs SCE. The highest concentration of $\operatorname{Trp}\left(1 \times 10^{-2} \mathrm{~mol} \mathrm{dm}^{-3}\right)$ revealed the most prominent initial increase in values of $\log (\mathrm{I})$ from the open-circuit value, generally displaying larger values for the duration of the test indicative of an increased corrosion current associated with the cathodic reaction.

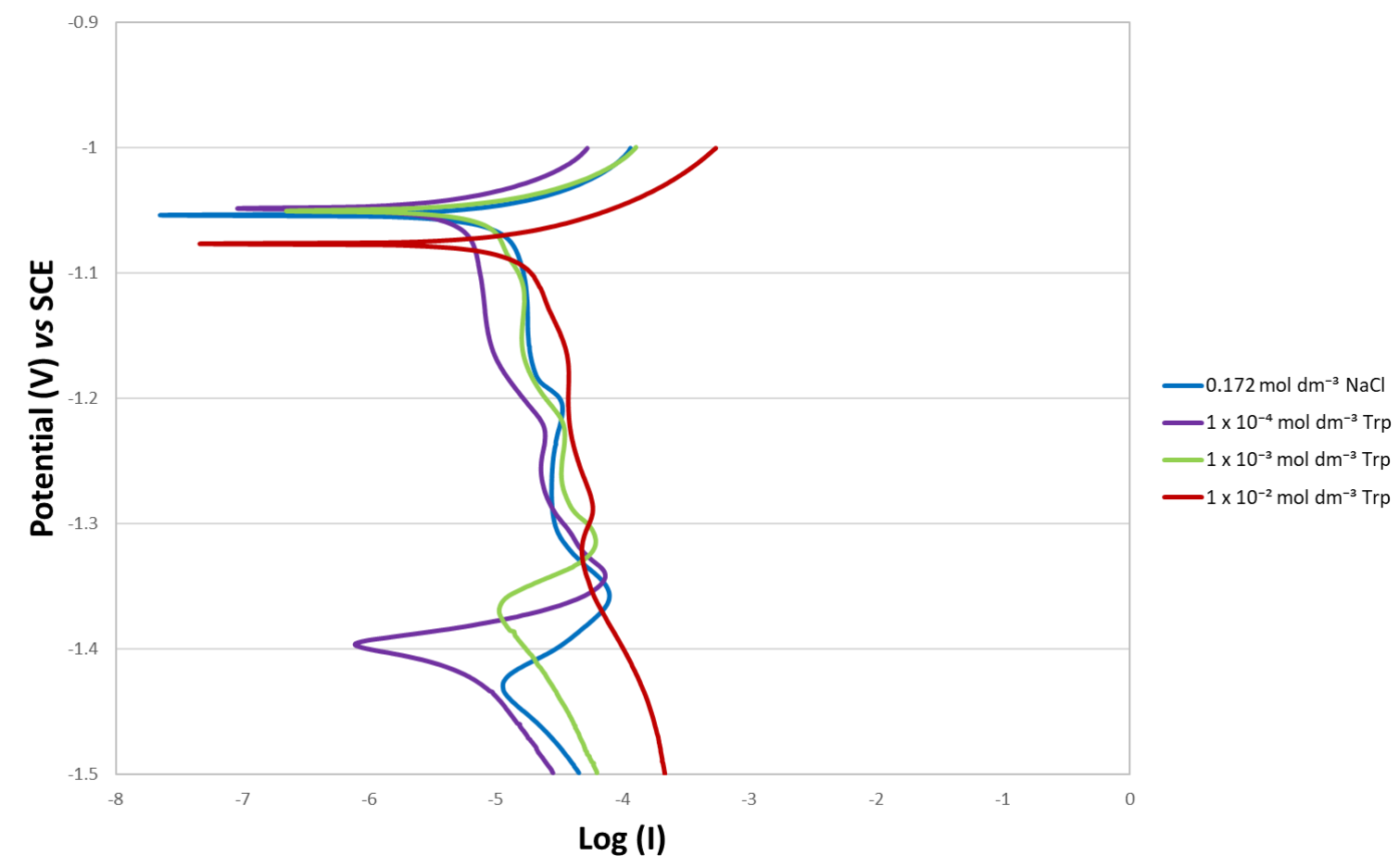

Figure 6.11 Plot of cathodic-going polarisation curves for the range of dosed inhibitor concentrations in $1 \% \mathrm{NaCl} \mathrm{pH} 7$ baseline electrolyte

The anodic-going sets of the polarisation curves are given in Figure 6.12. The plot demonstrated a general reduction in $E_{\text {corr }}$ for increasing concentrations of Trp. The most pronounced fall in $E_{\text {corr }}$ was observed for $1 \times 10^{-2} \mathrm{~mol} \mathrm{dm}^{-3} \mathrm{Trp}$. The curves showed that no significant impact to the 
anodic branch was visible, with the greatest Trp addition showing a marginally less sharp incline from its open-circuit reading. All of the curves appeared to merge with one another at approximately -0.85 vs SCE, following a steady and continual increase in log (I) as they were swept towards the $0 \mathrm{~V}$ vs SCE point.

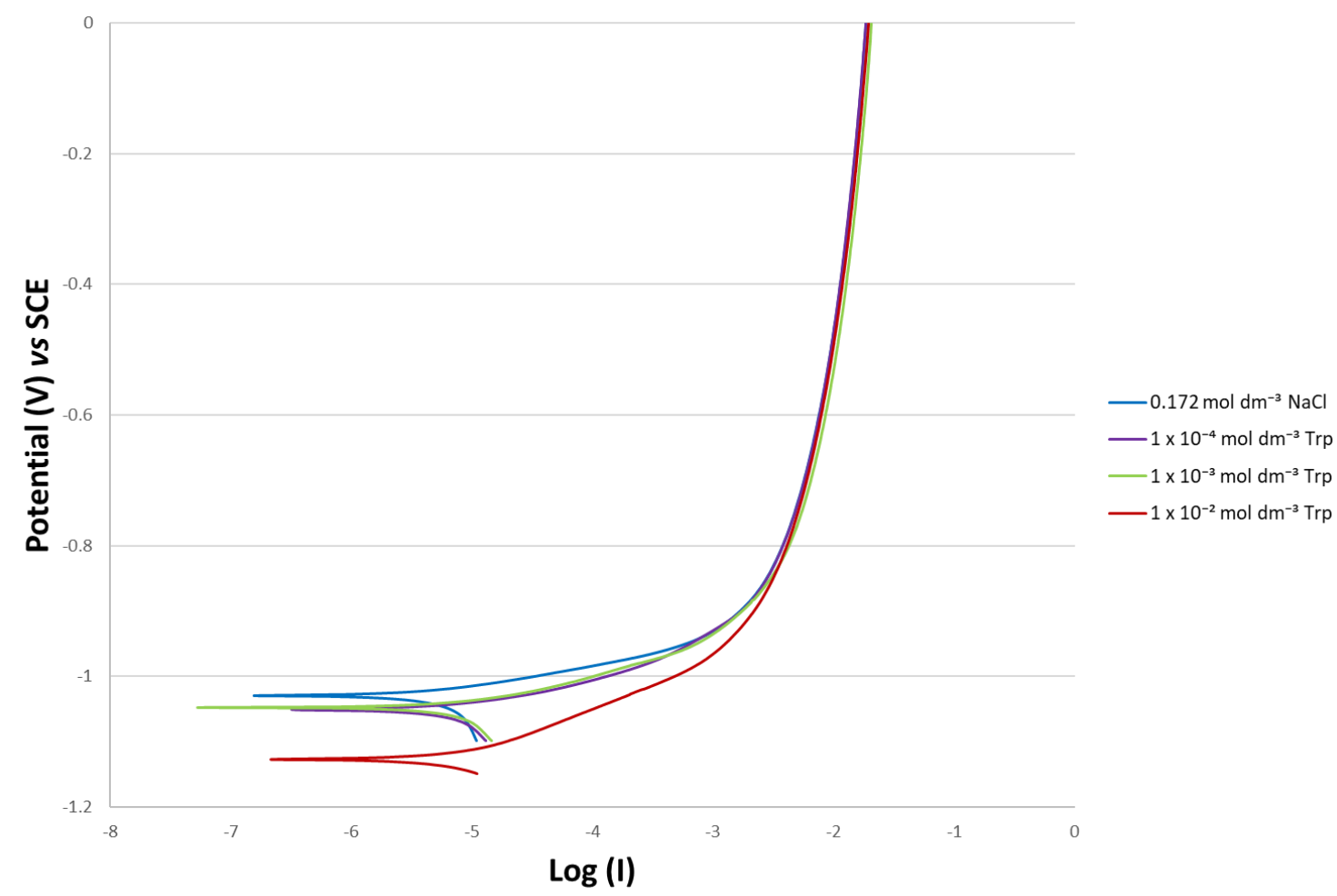

Figure 6.12 Plot of anodic-going polarisation curves for the range of dosed inhibitor concentrations in $1 \% \mathrm{NaCl}$ pH 7 baseline electrolyte

Further analysis in the form of Tafel-extrapolated metal loss was completed using the potentiodynamic polarisation data. This is presented in Table 6.2. Contrary to the SVET data, the reported trend from Tafel data exhibited an increase in metal loss for increasing quantities of Trp inhibitor into the electrolyte. Whilst SVET realised a beneficial reduction in metal loss at 1 $\times 10^{-2} \mathrm{~mol} \mathrm{dm}^{-3} \operatorname{Trp}$, the Tafel data did not, suggesting a sizeable increase in metal loss instead.

Table 6.2 Metal loss data from SVET and Tafel-extrapolation

\begin{tabular}{|c|c|c|}
\hline $\begin{array}{c}\text { Trp concentration } \\
\left(\mathrm{mol} \mathrm{dm}^{-3}\right)\end{array}$ & $\begin{array}{c}\text { SVET metal loss } \\
\left(\mathrm{g} \mathrm{m}^{-2}\right)\end{array}$ & $\begin{array}{c}\text { Tafel-derived metal } \\
\text { loss }\left(\mathrm{g} \mathrm{m}^{-2}\right)\end{array}$ \\
\hline 0 & 12.20 & 1.07 \\
\hline $1 \times 10^{-4}$ & 9.79 & 2.06 \\
\hline $1 \times 10^{-2}$ & 0.35 & 6.06 \\
\hline
\end{tabular}

In light of the other experiments conducted in the form of SVET and time-lapse, the polarisation results presented were not accordant with this data. The polarisation results showed no 
inhibition due to the presence of Trp but did provide evidence of film formation in the counterpart cathodic curves. This counterintuitive evidence obtained via polarisation curves may be due to the fact that potentiodynamic polarisation is a perturbing technique; this is because a voltage is driven across the circuit to polarise the sample with respect to a reference point. The other data in the form SVET, time-lapse microscopy, and even open-circuit potential allow the sample to freely corrode and may provide a more accurate, or consistent indicator of diminished corrosion. In addition to this, subsequent to the cathodic polarisation sweeps, visual inspection of the highest inhibitor concentration sample revealed a surface film highly similar to that observed upon completion of SVET experimentation. Despite the surface changes, the curve did not suggest any effects of this kind. It must be considered that the nature of this perturbing technique may have altered the mechanism of inhibition that was observed from the other data under free corrosion conditions. Furthermore, Tafel extrapolation has been suggested to provide less accurate information under conditions of localised corrosion [96], potentially contributing to the misaligned results.

\subsubsection{Surface analysis of corrosion inhibitor deposition via SEM and EDS}

At the conclusion of SVET experimentation, following immersion in $1 \times 10^{-2} \mathrm{~mol} \mathrm{dm}^{-3} \operatorname{Trp}$, a corroded sample was prepared to enable examination of the surface and any associated surface products. This was completed with the use of a Hitachi TM3000 Tabletop Scanning Electron Microscope, and the Bruker Quantax70 EDS system. 

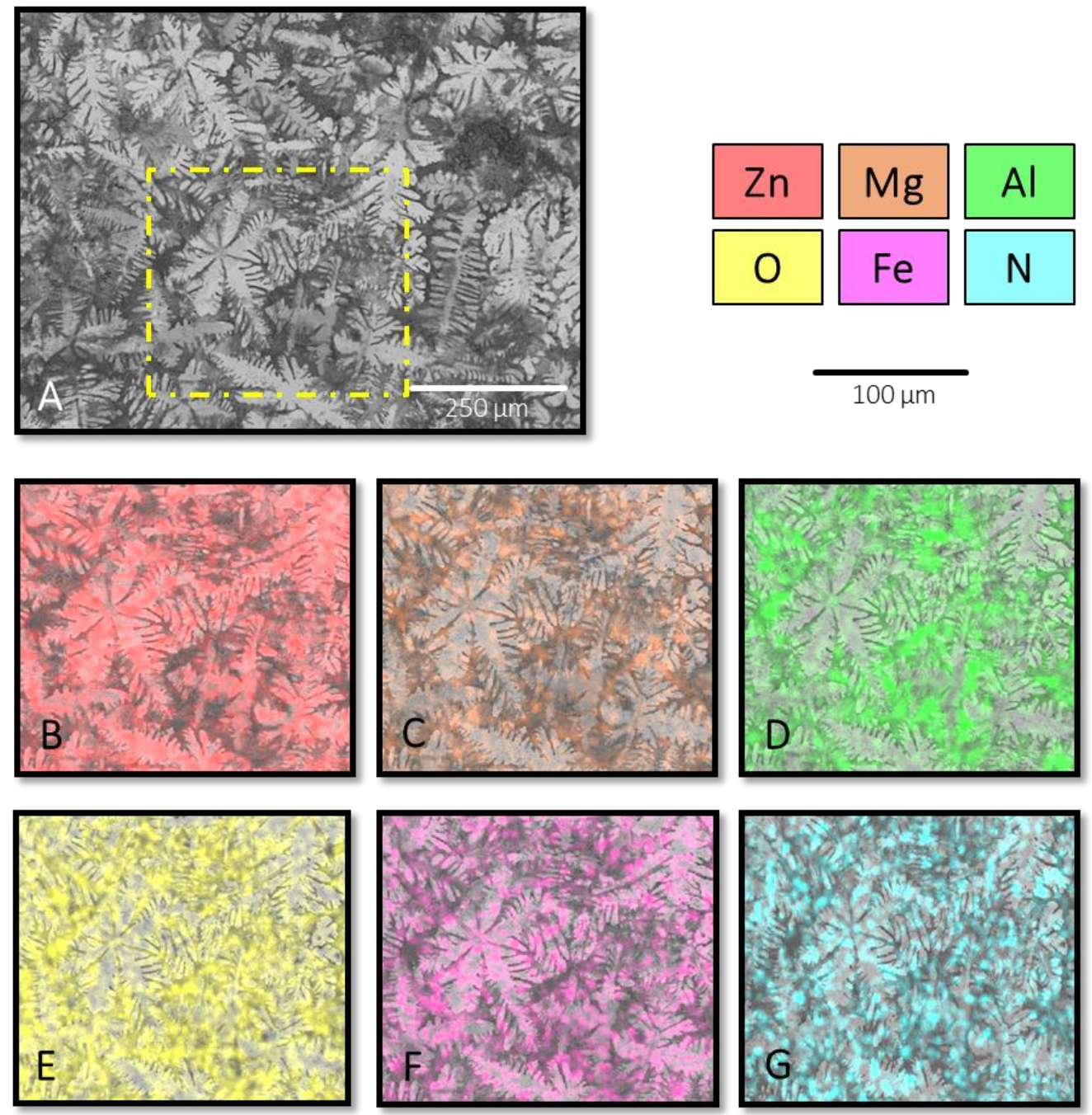

Figure $6.13\left(\right.$ A-G) SEM and EDS analysis of a ZMA sample following immersion in $1 \times 10^{-2} \mathrm{~mol} \mathrm{dm}^{-3}$ L-tryptophan

A representative image captured from the exposed surface is given in Figure 6.13 A. Elemental analysis in the form of EDS mapping is given for a selected area in Figure 6.13 B-G. The images demonstrated that the compositional elements of zinc, magnesium, and aluminium were unsurprisingly present on the surface, with zinc dominating the trio of elements detected. The presence of the individual constitutional elements may have simply been as a conventional corrosion product, or alternatively as a complex with the inhibitor. The presence of oxygen was also prominent on the surface, which was more than likely in the form of oxide-based corrosion product. Iron was also visible from the EDS map, which may be present due to accumulated iron in a galvanising bath following immersion of the strip steel substrate. Nitrogen was observable on the surface, which may have indicated Trp inhibitor interaction. As it has been suggested in other work that an excess of nitrogen atoms was in part responsible for Trp adsorption to a metal surface [106], that may explain its presence here. Whilst the overall evidence from this 
chapter indicated a precipitated surface film because of the Trp addition, the composition of this was not entirely clear.

\subsubsection{Validation of SVET-measured metal loss using gravimetric analysis}

Whilst SVET can provide somewhat unique data, the metal loss values derived from this technique are semi-quantitative due to not only its associated limitations, but also due to the assumptions involved in subsequent manipulation of the data. Therefore, gravimetric analysis was used to verify such data. The analysis was conducted according to the protocol set out in ISO 8407, as explained in 2.7. The samples were tested using two inhibitor concentrations, $1 \mathrm{x}$ $10^{-2} \mathrm{~mol} \mathrm{dm}^{-3}$ and $1 \times 10^{-4} \mathrm{~mol} \mathrm{dm}^{-3} \mathrm{Trp}$, in the baseline $1 \% \mathrm{NaCl} \mathrm{pH} 7$ electrolyte, and an uninhibited control counterpart. The samples of ZMA 2 were tested in their electrolyte, being immersed in solution for 24 hours to parallel the experimental time-frame utilised by SVET. Following the allocated immersion time, samples were removed and subjected to chemical cleaning cycles using a saturated glycine solution. The resulting comparison between SVET and gravimetric metal loss data is shown in Table 6.3.

Table 6.3 Comparison of measured metal loss values via gravimetric and SVET analysis

\begin{tabular}{|c|c|c|c|c|}
\hline $\begin{array}{c}\text { Trp concentration } \\
\left(\mathrm{mol} \mathrm{dm}^{-3}\right)\end{array}$ & $\begin{array}{c}\text { SVET metal } \\
\text { loss }\left(\mathrm{g} \mathrm{m}^{-2}\right)\end{array}$ & $\begin{array}{c}\text { Gravimetric metal } \\
\text { loss }\left(\mathrm{g} \mathrm{m}^{-2}\right)\end{array}$ & $\begin{array}{c}\text { SVET-measured } \\
\text { inhibition } \\
\text { efficiency }\end{array}$ & $\begin{array}{c}\text { Gravimetric- } \\
\text { measured } \\
\text { inhibition efficiency }\end{array}$ \\
\hline 0 & 12.20 & 10.95 & N/A & N/A \\
\hline $1 \times 10^{-4}$ & 9.79 & 11.94 & $19.8 \%$ & $-9 \%$ \\
\hline $1 \times 10^{-2}$ & 0.35 & 5.97 & $97.1 \%$ & $45.5 \%$ \\
\hline
\end{tabular}

It was immediate that the SVET values were lower in the case of both inhibitor additions, although this was not evident for the control data. In the case of $1 \times 10^{-2} \mathrm{~mol} \mathrm{dm}^{-3} \operatorname{Trp}$, the results confirmed a reduction in metal loss justifying an improved corrosion resistance. However, the lower inhibitor concentration demonstrated a greater extent of corrosion than the uninhibited counterpart. This corroborates the observations made upon comparison of the time-lapse microscopy data, indicating a diminished performance at this lower concentration. The primary reasons behind the observed differences may be the result of the SVET limitations and corresponding data. The SVET is limited in resolution and as such is only able to detect corrosion to a certain degree. The SVET will only detect localised corrosion features where anodes and cathodes are separated by a distance approximately equal to 1.5 times the scan height; lower level corrosion currents that do not intersect the SVET plane of scan of $100 \mu \mathrm{m}$ will not be registered. It is also limited in that the SVET realises a vector component of current density, and not the direct surface current density. Furthermore, the metal loss calculations 
assume that the progression of corrosion is constant between each hourly scan, which may not necessarily be the case.

Yet, additional factors may have affected the more substantial discrepancy visible for the highest Trp concentration. The data obtained using SVET was done so in situ, and readings are based on ionic current flux consequent of aqueous corrosion. This makes the readings highly sensitive, whereas gravimetric analysis is a bulk technique that measures corrosion activity in a samples' entirety, following said activity. This was performed using an analytical balance of $0.1 \mathrm{mg}$ readability, and given the scale of metal loss from the samples studied, it can be considered that these readings were subject to a degree of error. Moreover, any potential error margins may be amplified when scaling results to $\mathrm{g} \mathrm{m}^{-2}$ for means of consistency. The size of the exposed surface area between SVET and gravimetric testing differed, and also may have impacted the degree of corrosion or even the associated mechanism of inhibition. A combination of the above factors likely influenced the results given in Table 6.3. At the very least, a degree of confidence can be obtained from the results in that the performance ranking according to inhibitor variation was correct.

\subsubsection{Evaluation of the corrosion inhibition properties of L-tryptophan in a ZMA alloy}

The data presented within this chapter demonstrated that Trp indeed functioned as a corrosion inhibitor for the ZMA system for concentrations of $1 \times 10^{-3} \mathrm{~mol} \mathrm{dm}^{-3}$ or greater. The SVET current density maps revealed that at the lowest concentration of Trp, multiple point anodic features formed on the surface. These were observed to grow and coalesce over time, and the intensity of this activity was sustained throughout a large portion of the experiment. Increasing to the intermediate concentration still offered the formation of a multitude of point anodes on the surface, but with a reduced extent of growth, coalescence, and intensity. The greatest concentration instead offered very little SVET-resolved activity, coinciding with the most prolific extent of corrosion inhibition observed; such observations were clarified in the SVET anode analysis plot of Figure 6.5. The corresponding SVET Jat plots displayed the minimal progression of anodic activity at the highest Trp concentration, whereas the lowest concentration followed a similar trend to the control sample yet of a lower magnitude. The intermediate concentration provided a delayed onset of corrosion and a relatively lower magnitude; a fall at hour 16 and the lower values realised potentially indicated the length of time required to achieve stable inhibition via the accumulation of the surface film in a continuous manner. The continuity of the surface film associated with the inhibitor is likely related to the ability of Trp to inhibit corrosion; the highest concentration exhibited the greatest film continuity coupled with 
performance, whereas the less continuous layer for the intermediate concentration maintained a less effective performance. This is likely due to restriction of access to the surface in either a continuous or otherwise discontinuous manner. The time-lapse microscopy data echoed the performance ranking realised through SVET somewhat, showing improvement in corrosion resistance levels for increasing concentrations of Trp addition. Although, the time-lapse data instead revealed the $1 \times 10^{-4} \mathrm{~mol} \mathrm{dm}^{-3} \operatorname{Trp}$ addition to be less favourable than the control counterpart free of inhibitor. The time-lapse corroded area data generally correlated well with the SVET-measured metal loss values in terms of proportion. As stated above, the results obtained via SVET do have limitations associated with them, possibly underestimating the extent of corrosion; however, combined with the remaining data sets, corrosion performance ranking was substantiated with the support of gravimetric analysis. This can be further realised by the summary of metal loss values given in Table 6.4, obtained via the three techniques. The Tafel extrapolation largely underestimated the extent of corrosion, except for that of the highest Trp concentration. The performance ranking reached between SVET and gravimetric testing procedures was generally in agreement, with SVET realising an underestimated metal loss for the smallest inhibitor addition.

Table 6.4 Summary of metal loss data for different concentrations of L-tryptophan

\begin{tabular}{|c|c|c|c|}
\hline $\begin{array}{c}\text { Trp concentration } \\
\left(\mathrm{mol} \mathrm{dm}^{-3}\right)\end{array}$ & $\begin{array}{c}\text { SVET metal } \\
\text { loss }\left(\mathrm{g} \mathrm{m}^{-2}\right)\end{array}$ & $\begin{array}{c}\text { Gravimetric metal } \\
\text { loss }\left(\mathrm{g} \mathrm{m}^{-2}\right)\end{array}$ & $\begin{array}{c}\text { Tafel-derived } \\
\text { metal loss }\left(\mathrm{g} \mathrm{m}^{-2}\right)\end{array}$ \\
\hline 0 & 12.20 & 10.95 & 1.07 \\
\hline $1 \times 10^{-4}$ & 9.79 & 11.94 & 2.06 \\
\hline $1 \times 10^{-2}$ & 0.35 & 5.97 & 6.06 \\
\hline
\end{tabular}




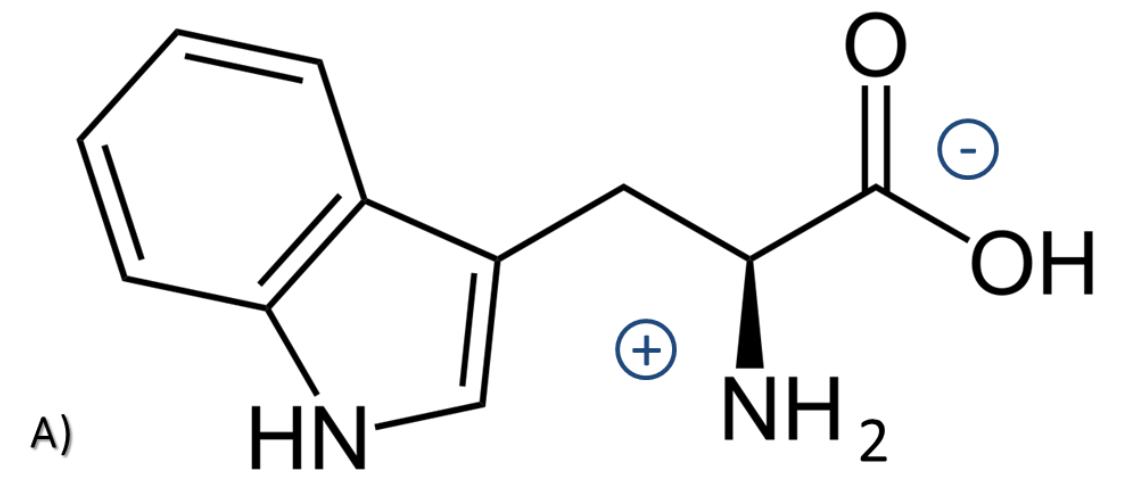

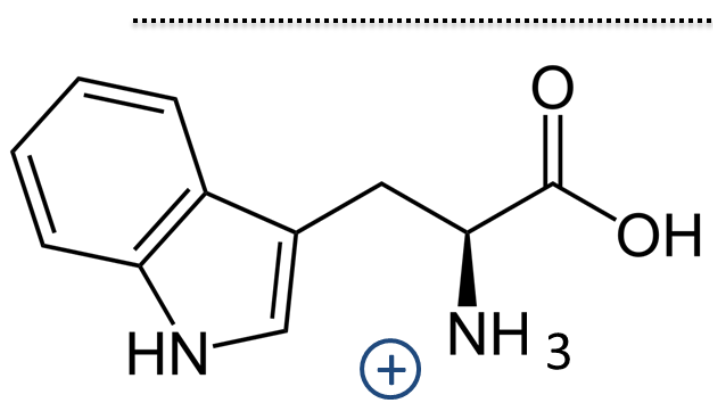

B)

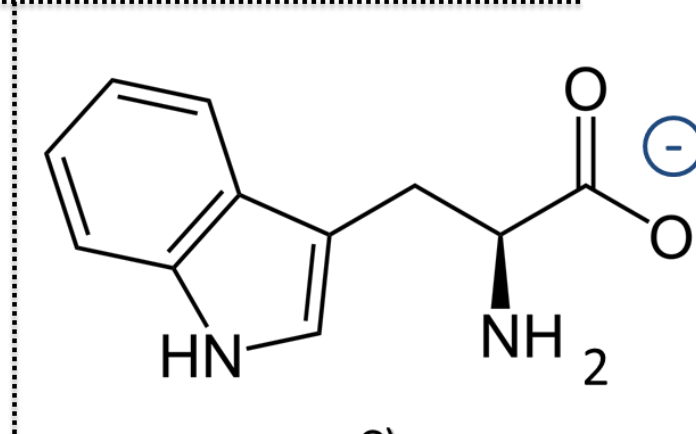

C)

Figure 6.14 Chemical structure of L-tryptophan in A) zwitterion, B) protonated, and C) deprotonated forms

Whilst the time-lapse microscopy data revealed that a film was precipitated onto the surface, seemingly within regions or phases supporting cathodic activity, it was not entirely clear as to how this transpired. It is well known that organic inhibitors adsorbed on metallic surfaces can do so via nitrogen and oxygen atoms [107], and the same can be considered in the case of Ltryptophan. The presence of the indole ring has also been attributed to its adsorption characteristics, as well as the sheer size of the molecule increasing its coverage ability comparative to other amino acids [106]. Work considering copper in acidic media exhibited appreciable degrees of efficiency in conjunction with L-tryptophan [108]; this was also the case for low carbon steel, which found that Trp acted as an effective cathodic inhibitor within acidic conditions [109]. The reason that Trp is so effective in acidic conditions may be due to $\mathrm{pH}$ relative to the isoelectric point. Bereket et al. stated that amino acids possess greater inhibition effects with increasing distance from their isoelectric point, consequent of higher dipole moments [34]. L-tryptophan exists as a zwitterion structure at median $\mathrm{pH}$ ranges. The zwitterion state of the molecule, as depicted in Figure 6.14 A, means that the environment can essentially dictate the behaviour of the Trp within the given $\mathrm{pH}$ threshold. However, outside the pKa ranges of $\mathrm{pKa}_{1} 2.38$ for the carboxylic acid, and $\mathrm{pKa}_{2} 9.39$ for the amine group [110], it behaves as a cation or an anion (indicated in Figure 6.14 B and C), respectively. As such, at more extreme $\mathrm{pH}$ conditions, Trp can be understood to function more effectively as an inhibitor. 
Within neutral, chloride-containing environments, it was reported that an amino acid present as a zwitterion should undergo enhanced adsorption due to the presence of chloride ions [107]. The author postulated that the chloride ions present at active sites on a metal surface underwent stabilised adsorption leading to the decreased corrosion rate. This would result in the adsorption of the cationic form of Trp. As this would occur in the vicinity of active sites, it would enable Trp to act as an anodic inhibitor, explaining the moderate coverage observed in this region for the time-lapse experiments. As the $\mathrm{pH}$ environment local to the site of the cathode would be higher than that of the bulk pH 7 solution, anionic behaviour of the inhibitor may have transpired. This could lead to the reaction with metal cations emanating from an anodic site, and thus to anodic inhibition and the consequent formation of a film proximal to the anode. This is supported by other work conducted on a pure zinc substrate [111]; Trp was observed to precipitate local to the site of metal dissolution at higher $\mathrm{pH}$ conditions, where Trp is in anionic form. This is suggestive of a metal-Trp complex. Alternatively, on the proviso that in the stages leading up to the addition of inhibitor that the corrosion products on the surface were composed of a metal oxide or hydroxide, the isoelectric point of the product would be greater than the bulk $\mathrm{pH}$; this would render the substrate surface positively charged, enabling the Trp in anionic form to readily adsorb onto the surface. Furthermore, the beneficial properties of ZMA are in part associated with the rapid dissolution of magnesium and the ensuing corrosion products [72], [74], so it is entirely possible that this aided the presence of Trp in its anionic form.

Whilst inhibitor deposition was evidenced to a small extent in the anodic region, the gathered evidence demonstrated a deposited layer predominantly within regions considered as sites of cathodic activity, which suggests that inhibitor deposition may have occurred mostly by other means. Additionally, the $\mathrm{E}_{\text {corr }}$ values observable from the open-circuit potential testing are concurrent with that of the Evans diagram for cathodic protection. The work surrounding ZMA alloys has demonstrated that the presence of magnesium-based corrosion product is understood to buffer the $\mathrm{pH}$ though the formation of magnesium carbonate compounds [72]; this may in fact discourage deprotonation of the amino group, or even the local adsorption of the anionic form of Trp to the substrate, to some extent. Other mechanisms may have aided Trp deposition; the metal cations emanating from the anodic site, diffusing towards the cathodic region, may have reacted with Trp anions to form a metal-Trp complex. In a study considering a complex of $\mathrm{Zn}(\mathrm{II})$ and an assortment of amino acids, that of $\mathrm{Zn}$ (II)-tryptophan was realised to be poorly soluble [112]. This event could occur to form a sparingly soluble film at the site of the cathode, hindering mass transport of oxygen to the surface, and thereby limiting the 
cathodic half reaction. However, it is equally possible that the abundance of negatively charged ions generated at the cathodic site reacted with cationic Trp to form a product that ultimately precipitated at the cathode; this too would bring about retardation of the cathodic reaction. The surface film formation was supported by the SEM-EDS results given in Figure 6.13, but a specific film or complex could not be distinguished from the data. In the case of Video 6.3, introduction of the Trp inhibitor illustrated the formation of a film in the cathodic area at the greatest distance away from the anode. This film was then seen to extend over the cathodic region rapidly and in a hemispherical profile towards the anode. There was also evidence of some film deposition towards the anodic site itself, but in a much more moderate manner. This strongly indicated that the cathodic process instigated either the formation of a metal-Trp complex, or adsorption at the metal surface.

\subsection{Conclusions}

The influence of an amino acid in the form of L-tryptophan at varying concentrations on a commercially produced ZMA alloy has been considered. The data clarified that Trp additions ranging from $1 \times 10^{-3} \mathrm{~mol} \mathrm{dm}^{-3}$ to $1 \times 10^{-2} \mathrm{~mol} \mathrm{dm}^{-3}$ provided appreciable improvements in corrosion resistance levels, especially so for the latter concentration. However, for $1 \times 10^{-4} \mathrm{~mol}$ $\mathrm{dm}^{-3}$, the control counterpart offered favourable performance comparatively. An inverse relationship between the inhibitor concentration and the extent of corrosion was accordingly realised. The data obtained using SVET revealed that the intensity of anodic attack in the form of multiple point anodes, decreased for greater inhibitor concentrations. The growth, coalescence, and intensity of anodic activity diminished following an increase in Trp addition, with $1 \times 10^{-2} \mathrm{~mol} \mathrm{dm}^{-3} \mathrm{Trp}$ showing little activity beyond the early stages of the experiment. SVET metal loss data revealed significant reduction in values associated with the largest inhibitor addition, the extent of which was more gradual for the intermediate concentration. The time-lapse microscopy data reaffirmed this trend, exhibiting progressively restricted anodic growth as Trp additions were increased. The area-averaged anodic current density plots demonstrated a reduced magnitude of values as the inhibitor addition was increased, with the highest concentration demonstrating the rapid decline in anodic activity beyond the initial stages. The intermediate concentration evidenced a secondary fall in anodic progression later on in the experiment; this may have coincided with sufficient time to form a more continuous surface film, limiting the rate of corrosive attack. The time-lapse corroded area analysis data revealed a performance ranking similar to that of SVET with respect to inhibitor concentration, excluding that of the lowest concentration. The same time-lapse analysis realised a lessened extent of corrosion for the intermediate concentration of Trp, potentially explained by the two- 
dimensional limitation of the technique. The SVET-derived metal loss values were contrasted to that of gravimetric analysis; the comparison exhibited a matching trend, but SVET-generated values were lower comparative to gravimetric tests. The extent of the discrepancy between techniques was notably greater for the $1 \times 10^{-2} \mathrm{~mol} \mathrm{dm}^{-3} \mathrm{Trp}$ addition, potentially rooted in the limitations associated with both techniques. Contrary to this, the Tafel-extrapolated metal loss was more accurate for the highest Trp concentration, whilst the remaining tests were then more divergent.

The addition of the amino acid, L-tryptophan, was observed to provide favourable changes in corrosion properties, attributable to the precipitation of a film on the surface of the alloy. As visible from the time-lapse sequences, whilst a conventional corrosion product was apparent, a superfluous film was increasingly present in line with larger additions of inhibitor. As best demonstrated by the highest concentration, this film originated in areas of cathodic activity and was observed to accumulate over time becoming increasingly darkened. This was discerned to cover the cathodic region in a rapid manner, but also to form proximal to the anode as the experiment progressed. The increased coverage was associated with the declining growth rate of the focal anodic feature. This can be understood through hindering the adsorption of oxygen at the surface, thereby impeding the rate of cathodic reaction. A restriction to the cathodic reaction will impact upon the anodic reaction and thus, slow the overall rate of corrosion.

The post-corrosion analysis performed using SEM-EDS revealed the presence of nitrogen and oxygen in addition to the compositional elements, potentially indicating the deposition of the inhibitor. However, this was by no means definitive and further work is required for a more certain characterisation. The potentiostat-based open-circuit potential testing revealed a fall in $\mathrm{E}_{\text {corr }}$ coincident with an increase in concentration of inhibitor; the results aligned with that of the Evans diagram for cathodic protection, indicating that the Trp inhibitor should offer a cathodic inhibition response. The polarisation curves exhibited results discordant with the remainder of the data sets, instead showing that the greatest Trp addition revealed the largest values of overall log (I) for the cathodic sweep. This was relayed in the Tafel-extrapolated metal loss data also. Accordingly, it has been proposed that the perturbation associated with potentiodynamic polarisation impacted upon the mechanism of action for the inhibitor, thereby affecting the outcome comparative to the other freely corroding techniques utilised. The presence of a continuous film apparent on the surface following visual inspection also supports this notion. The corresponding anodic polarisation sweep revealed little effect regardless of inhibitor concentration, excluding a shift in open-circuit potential value. 
The possible mechanisms of inhibition for Trp have been discussed. Time-lapse microscopy experiments indicated the adsorption of a film on the ZMA surface. However, the specific action associated with the protective film is unclear. It may well be the case that localised changes to $\mathrm{pH}$ permitted the formation of a sparingly soluble complex composing metal cations and inhibitor anions, or negatively charged ions from the cathodic site and cations of Trp. Alternatively, the overall charge at regions of the bulk surface permitted the zwitterion to adsorb as a film and protect the surface. Moreover, the presence of adsorbed chloride ions at the interface of the metal may have enhanced adsorption of Trp cations. Nevertheless, the in situ time-lapse observations demonstrated predominant deposition at the cathodic site, followed by moderate precipitation at the focal anode for the highest concentration.

Ultimately, L-tryptophan has been shown as a corrosion inhibitor with environmentally-friendly prospects for the growing use of ZMA, but highlights the importance of further work to clarify the effect of $\mathrm{pH}$ and thus, its application capabilities. 


\section{Chapter 7 Examination of the inhibition properties of a rare earth element during corrosion of a zinc-magnesium-aluminium (ZMA) galvanising alloy}

\subsection{Introduction}

Within this chapter, a rare earth metal in the form of $\mathrm{CeCl}_{3}$ is used as a species of corrosion inhibitor addition, as a prospective alternative to chromium-based compounds. Chapter 5 demonstrated not only the effectiveness of sodium phosphate as a chromate-free inhibitor within the ZMA system, but the likelihood of it being classified as an anodic inhibitor. Continuing with this theme, cerium(III) chloride is contrarily thought to act as a cathodic inhibitor [40]. Once again, to represent the ZMA system, a zinc alloy containing small additions of $\mathrm{Mg}$ and $\mathrm{Al}$ was used as the sample of choice. This is the same alloy as used in the two previous chapters and details of the alloy are outlined in the following section, within Table 7.1. The reason for employment of this particular ZMA alloy is based in its industrial relevancy as well as its balanced level of performance seen within Chapter 3.

This investigation is founded in the potential that an inhibitor of this nature may have for this class of ZMA alloy. Bethencourt et al. concluded that the optimal concentration of cerium salts for inhibition purposes varies considerably according to alloy [39]. As such, the overall objectives were to establish the extent of corrosion inhibition offered by $\mathrm{CeCl}_{3}$ on a ZMA surface, and to understand the mechanisms underpinning the observed behaviour. This was conducted through additions of the rare earth inhibitor to the electrolyte solution at a range of concentrations, and subsequent testing of corrosion behaviour under immersion conditions. Despite its classification as a rare earth metal, cerium is considered to be akin to copper in terms of earth abundance [39].

The SVET has been employed to map the changes in localised surface corrosion events according to the variation in concentration of inhibitor introduced. Accordingly, the SVET data was used to also compare relative performances of the ZMA alloy via estimated metal loss values and area-averaged anodic current density plots. Time-lapse optical microscopy has proved valuable in visualisation of corrosion mechanisms on a microstructural level and was utilised for that same purpose within this chapter. Open-circuit potential and potentiodynamic polarisation experiments were carried out in conjunction with a potentiostat to further clarify the transpiring inhibition mechanisms. The series of testing within this chapter was performed with respect to the varying concentrations of $\mathrm{CeCl}_{3}$ inhibitor additions. 


\subsection{Experimental}

\subsubsection{Metallic specimens}

The ZMA alloy coated steel utilised throughout this investigation is detailed in Table 7.1. This was supplied by the French Corrosion Institute and manufactured under production line conditions.

Table 7.1 Composition of the zinc-magnesium-aluminium alloy under scrutiny

\begin{tabular}{|c|c|c|c|c|}
\hline Sample & Zn (wt\%) & Mg (wt\%) & Al $(w t \%)$ & $\begin{array}{c}\text { Coating thickness } \\
(\mu \mathrm{m})\end{array}$ \\
\hline ZMA 2 & 96 & 2 & 2 & 10 \\
\hline
\end{tabular}

\subsubsection{Sample preparation}

Following the procedures set out in 2.1.2.1, samples of ZMA 2 were cut into coupons of $20 \mathrm{~mm}$ x $20 \mathrm{~mm}$ dimensions, prior to mounting. Mounted samples were subsequently polished to a 1 $\mu \mathrm{m}$ surface finish, followed by cleaning with distilled water and then ethanol. Samples were etched using a solution of Nital, and the above cleaning practice repeated. The PTFE taping procedures for masking of the sample surface were identical to those outlined previously in 2.1.2, exposing areas of $c a .100 \mathrm{~mm}^{2}$ and $0.785 \mathrm{~mm}^{2}$ for the SVET and time-lapse experiments, respectively.

Samples undergoing potentiostat-based electrochemical testing, following metallographic preparation, were carefully removed from their mount. This was to allow the alloy coupon to fit securely within the custom-designed sample holder, exposing a sample area of $0.95 \mathrm{~mm}^{2}$ when fitted. This apparatus is described fully in sections 2.5-2.6.

\subsubsection{Experimental methodologies}

The time-lapse, SVET and electrochemical experiments were all conducted under immersion conditions. The electrolyte used for these immersion conditions was an aerated $1 \% \mathrm{w} / \mathrm{v} \mathrm{NaCl} \mathrm{pH}$ 7 electrolyte in all cases. This was considered the baseline electrolyte and was used as the control, or solution free of corrosion inhibitor. Dosing additions of the $\mathrm{CeCl}_{3}$ inhibitor were introduced to this baseline electrolyte, ranging from a concentration of $1 \times 10^{-4}$ to $1 \times 10^{-2} \mathrm{~mol}$ $\mathrm{dm}^{-3} \mathrm{CeCl}_{3}$. The dosed electrolytes were used from the onset of the SVET and electrochemical tests, however this differed for the time-lapse experiments. The time-lapse testing was initiated in the baseline $1 \% \mathrm{w} / \mathrm{v} \mathrm{NaCl} \mathrm{pH} 7$ solution, and once a sufficient anodic feature was visible, additions of the $\mathrm{CeCl}_{3}$ were introduced. Any and all adjustments to the electrolyte $\mathrm{pH}$ were performed by droplet additions of dilute $\mathrm{NaOH}$ or $\mathrm{HCl}$ solution. 
The SVET experiments were of 24-hour duration, beginning from the point of electrolyte introduction and being scanned hourly therein. The SVET setup, calibration and data analysis has been performed in the same fashion as described in section 2.2. A minimum of two repeat scans for each concentration of inhibitor was conducted to ensure the accuracy of the gathered results. The potentials measured during open-circuit potential (OCP) and polarisation tests were all recorded versus a saturated calomel electrode (SCE). Polarisation tests carried out on the ZMA 2 samples across the range of inhibitor concentrations were swept from -1.4 V up to $0 \mathrm{~V}$, at a sweep rate of $1 \mathrm{mV} \mathrm{s}^{-1}$. Further details of the methods utilised above are stated in Chapter 2.

\subsection{Results and discussion}

\subsubsection{Corrosion inhibition effects of $\mathrm{CeCl}_{3}$ on a ZMA alloy via SVET}

The SVET was used to examine the impact of $\mathrm{CeCl}_{3}$ as a corrosion inhibitor on a ZMA coated steel sample, as specified Table 7.1. Samples were allowed to freely corrode in the $1 \% \mathrm{w} / \mathrm{v} \mathrm{NaCl}$ electrolyte, neutralised to $\mathrm{pH} 7$, including additions ranging from $1 \times 10^{-4}$ up to $1 \times 10^{-2} \mathrm{CeCl}_{3} \mathrm{~mol}$ $\mathrm{dm}^{-3}$. This enabled the distribution of normal current density on a freely corroding surface to be mapped over a given time and across the range of dosed inhibitor concentrations. The maps displayed in Figure 7.2-7.5 span from the scan taken at the onset of the experiment at hour 0, up to hour 24. Additionally, Figure 7.1 reveals an estimation of the sample metal loss produced via manipulation of data, outlined in 2.2.5. The experiment conducted within Chapter 3 followed the same procedure and conditions used for this section of work, and as such, the data obtained for ZMA 2 in a 1\% w/v NaCl pH 7 electrolyte has been included as a point of reference for the relative performance of the inhibitor species (Figure 7.2). Figure 7.1 indicated an immediate $39 \%$ reduction in the metal loss values upon introduction of the $\mathrm{CeCl}_{3}$ at a concentration of $1 \times 10^{-4} \mathrm{~mol} \mathrm{dm}^{-3}$ comparative to the control sample. Increasing the inhibitor concentration to $1 \times 10^{-3} \mathrm{~mol} \mathrm{dm}^{-3}$ demonstrated a reduction of $92 \%$ in comparison with that of the uninhibited sample. However, increasing the inhibitor concentration by an order of magnitude up to $1 \times 10^{-2} \mathrm{CeCl}_{3} \mathrm{~mol} \mathrm{dm}^{-3}$ yielded little improvement on the previous inhibitor concentrations, revealing only a minor reduction in the value of metal loss comparatively. On the whole, this indeed showcased the ability of $\mathrm{CeCl}_{3}$ to act as an inhibitor through the reduction in sample metal loss calculations. Whilst the introduction of inhibitor species up to $1 \mathrm{x}$ $10^{-3} \mathrm{~mol} \mathrm{dm}^{-3}$ demonstrated a great impact on the metal loss values as the concentration was altered, the highest concentration did not offer an equivalent effect. The less significant upturn in performance seen for $1 \times 10^{-2} \mathrm{CeCl}_{3} \mathrm{~mol} \mathrm{dm}^{-3}$ compared to the second highest inhibitor 
quantity was suggestive of a threshold at which point increasing the quantity of inhibitor provides diminishing returns. This could potentially indicate a performance limit for $\mathrm{CeCl}_{3}$ as a corrosion inhibitor in this system. Likewise, this could instead suggest an onset time for the inhibitor to react sufficiently under these conditions.

The current density SVET maps given in Figure 7.2-7.5 show the distribution of anodic and cathodic activity resolved on the surface for the experiments conducted, including the range of inhibitor additions. Figure 7.3 displayed low levels of activity in the form of several point anodes at hour 0 . Some of these anodes persisted, showing little change, whereas others progressed and increased in size and intensity. Anodes were seen to progress on the surface throughout the experiment, where some point anodes coalesced and formed larger islands of anodic activity, and others persisted independently. The plots for $1 \times 10^{-3} \mathrm{CeCl}_{3} \mathrm{~mol} \mathrm{dm}^{-3}$ in Figure 7.4 demonstrated a noticeable change in surface corrosion events, with little activity visible from hour 0 . As the experiment developed, a small number of highly localised anodes were apparent, and their growth was visibly restricted in comparison with the experiments for the control and lowest $\mathrm{CeCl}_{3}$ additions. Whilst outward radial progression of anodes was not visible in Figure 7.3, it was evident in the $1 \times 10^{-3} \mathrm{CeCl}_{3} \mathrm{~mol} \mathrm{dm}^{-3}$ maps that anodic activity was much more stable overall. The clear reduction in anodic activity linked suitably with the trend from the metal loss calculations, where a considerable increase in corrosion resistance was observed for higher concentrations of inhibitor. From Figure 7.5, very little activity was resolved by the SVET at hour 0 . The plot at hour 4 proceeded to show the formation of several localised anodes, which endured until the end of the experiment. The characteristic radial progression of anodes was once again discernible, and present in more than one site. The anodic activity throughout the experiment appeared very stable, and mostly independent, with a single anode exhibiting coalescence between hours 20-24. Whilst there were similarities between Figure 7.5 and Figure 7.4, the magnitude of the anodic-going current density realised by SVET was reduced for the highest concentration of inhibitor on the whole. The SVET-derived maps fully matched the trend observed from the estimated metal loss results across the range of $\mathrm{CeCl}_{3}$ inhibitor concentrations. The agreement between data sets reveals the ability of $\mathrm{CeCl}_{3}$ to act as a corrosion inhibitor, improving performance with increasing concentrations seemingly in a nonlinear fashion and up to a threshold. Once SVET experimentation had elapsed, upon inspection of the samples it was noted that a distinct film had formed on the surface for the two highest $\mathrm{CeCl}_{3}$ concentrations. Whilst this was less so the case for the $1 \times 10^{-4} \mathrm{~mol} \mathrm{dm}^{-3} \mathrm{CeCl}_{3}$ experiments, the observed films had an evident gold-yellow hue. This appearance is apparent 
for cerium(III) oxide, and may provide an indication of the presence of this compound as a surface product following corrosion events.

The SVET anode analysis plotted in Figure 7.6 upheld the evidence indicating that the lowest concentration of $\mathrm{CeCl}_{3}$ provided less corrosion protection than observed for the higher concentrations. However, the intermediate inhibitor concentration suggested greater benefit to corrosion resistance compared to the $1 \times 10^{-2} \mathrm{~mol} \mathrm{dm}^{-3}$ addition. This was not the case when considering the SVET current density maps also; these exhibited a lessened overall intensity of anodic activity and fewer number of established sites on the surface at the higher concentration.

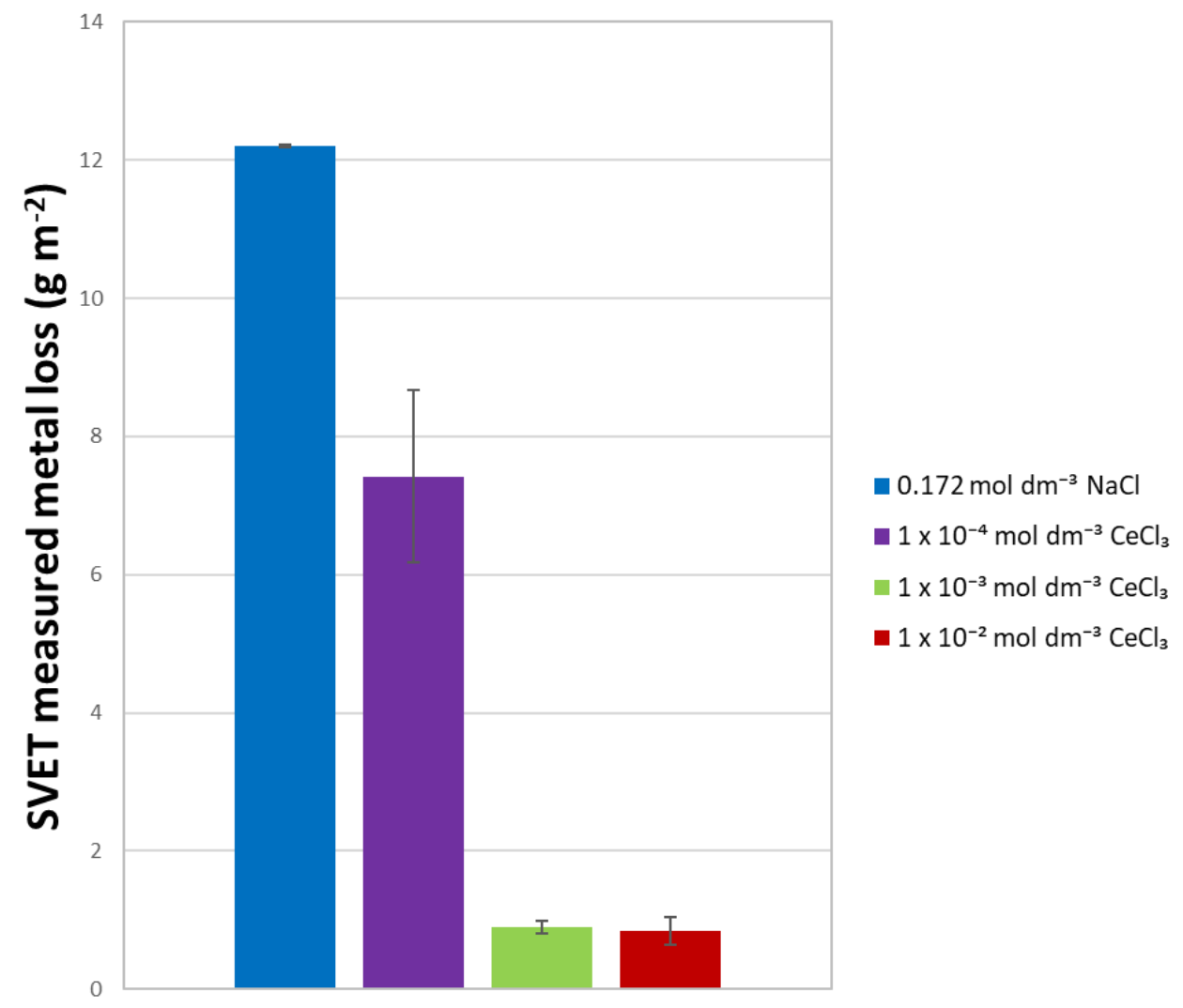

\section{Concentration of $\mathrm{CeCl}_{3}\left(\mathrm{~mol} \mathrm{dm}^{-3}\right)$}

Figure 7.1 SVET estimated metal loss over 24-hour experiment time for ZMA 2 in $1 \% \mathrm{NaCl} p H$ with varying concentrations of $\mathrm{CeCl}_{3}$ inhibitor

The area-averaged anodic current density values $\left(\mathrm{Ja}_{\mathrm{t}}\right)$ as a function of time plotted in Figure 7.7 highlighted the rate of anodic progression with time for the duration of each experiment and for each parameter of interest. The control experiment showed a sharp climb in Ja from the onset, which diminished by hour 1 . The introduction of $\mathrm{CeCl}_{3}$ at the lowest concentration displayed a similar trend but to a lesser extent, and revealed a reasonable variation in these 
values across the experimental time-frame. The values of $\mathrm{Ja}_{t}$ increased with time for the most part, which coincided with a larger proportion of the surface becoming visibly occupied with anodic features. The highest concentrations of $1 \times 10^{-3}$ and $1 \times 10^{-2} \mathrm{~mol} \mathrm{dm}^{-3} \mathrm{CeCl}_{3}$ did not exhibit the stark $\mathrm{Ja}_{t}$ increase visible in the other samples from the beginning of the experiment up to hour 1 ; instead they displayed a very mild reduction. The plots for the higher concentrations appeared to be much more consistent over time, especially so for the $1 \times 10^{-2}$ mol dm${ }^{-3} \mathrm{CeCl}_{3}$. The latter end of the experiment displayed a gradual rise in $\mathrm{Ja}_{\mathrm{t}}$ for the $1 \times 10^{-3}$ mol dm${ }^{-3} \mathrm{CeCl}_{3}$, which was suggestive of a decline in the effectiveness of inhibition. The highest $\mathrm{CeCl}_{3}$ addition did not indicate any signs of undulation towards the latter part of the experiment; this could not be appreciated from the metal loss values or even the normal current density plots alone. This may relate to a more pronounced corrosion inhibition effect at $1 \times 10^{-2} \mathrm{~mol} \mathrm{dm}^{-3} \mathrm{CeCl}_{3}$, but over a time-frame beyond the given length of the experiment. Therefore, whilst the estimated metal loss results from Figure 7.1 displayed only minor differences between the two highest inhibitor concentrations, the corrosion performance associated with the $1 \times 10^{-2} \mathrm{~mol} \mathrm{dm}^{-3} \mathrm{CeCl}_{3}$ may be more prolonged than that of the intermediate concentration. Considering this further, the performance observed for the highest concentration may be somewhat limited in terms of restraining overall metal lost from the sample visible during the length of experiment; it may actually possess longer-term effects than that seen within this 24-hour time-frame. Overall, it can be seen from SVET that the measured current density values were reduced following the increasing concentrations of $\mathrm{CeCl}_{3}$ inhibitor, demonstrating its ability to inhibit corrosion under these circumstances. 

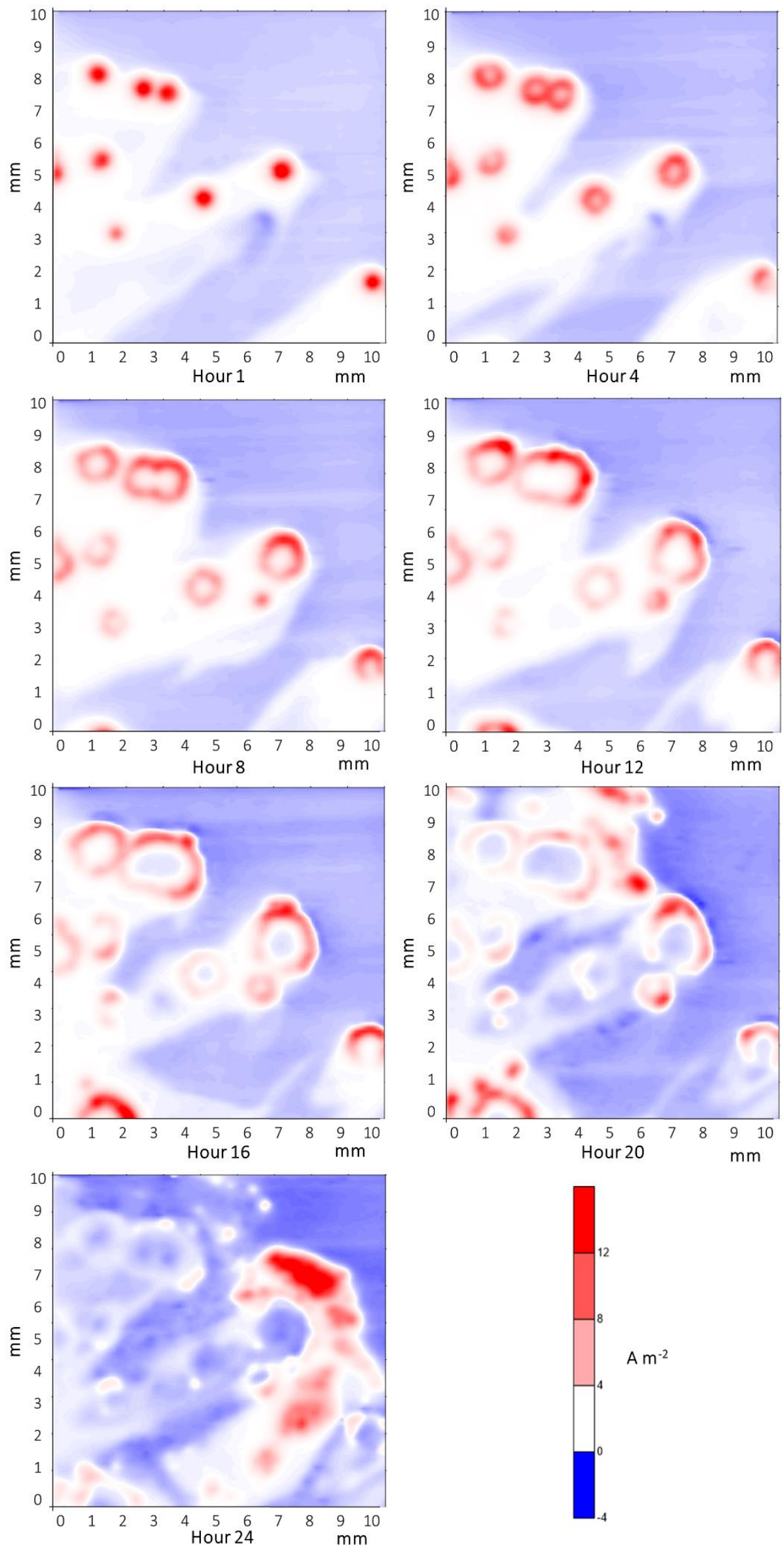

Figure 7.2 SVET normal current density maps of ZMA 2 in $1 \% \mathrm{NaCl} p H 7$ 

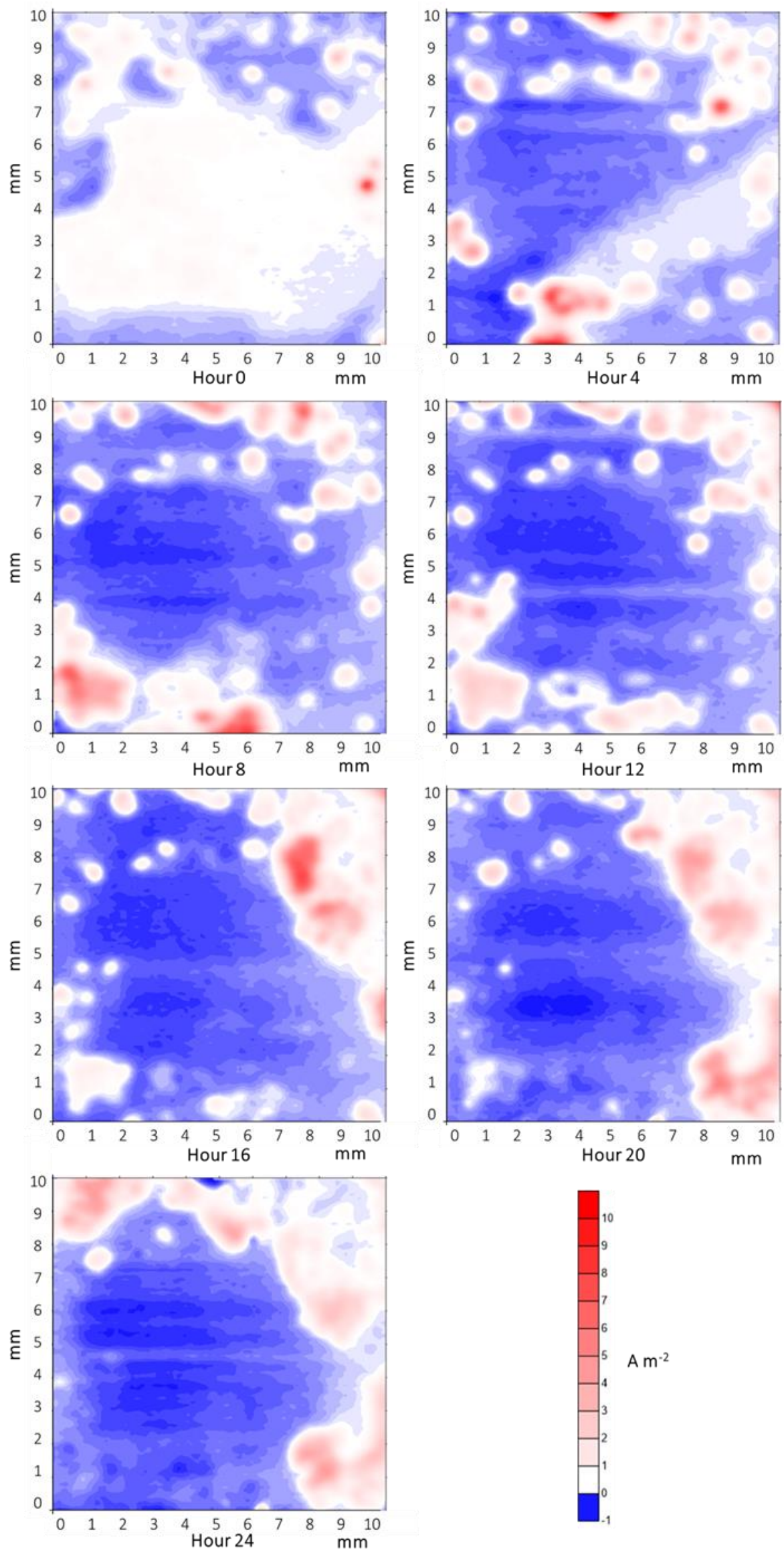

Figure 7.3 SVET normal current density maps of ZMA 2 in $1 \% \mathrm{NaCl} \mathrm{pH} 7$, including $1 \times 10^{-4} \mathrm{~mol}^{-1}$ $\mathrm{dm}^{-3} \mathrm{CeCl}_{3}$ 

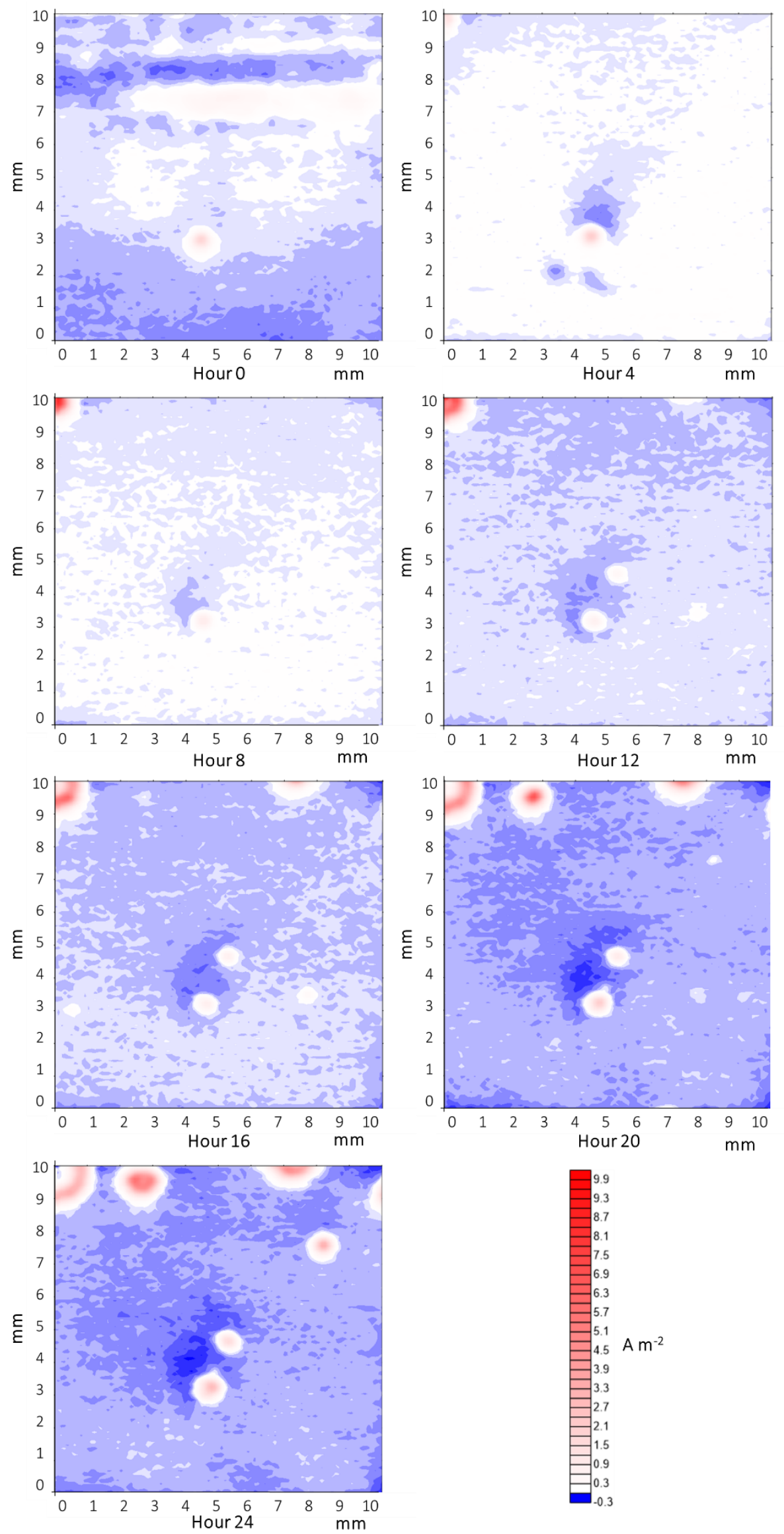

Figure 7.4 SVET normal current density maps of ZMA 2 in $1 \% \mathrm{NaCl} \mathrm{pH} 7$, including $1 \times 10^{-3} \mathrm{~mol}$ $\mathrm{dm}^{-3} \mathrm{CeCl}_{3}$ 

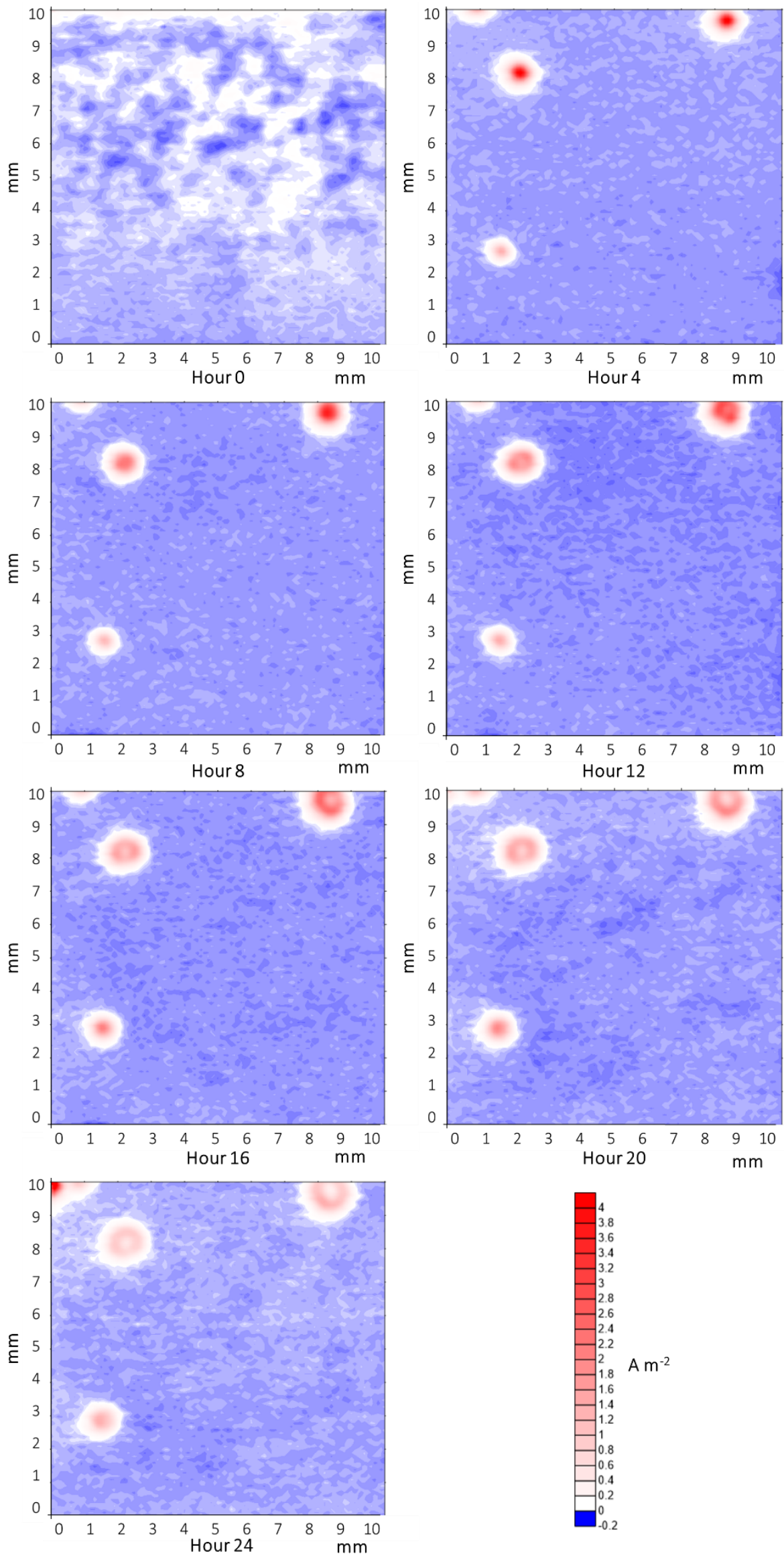

Figure 7.5 SVET normal current density maps of ZMA 2 in $1 \% \mathrm{NaCl} p H 7$, including $1 \times 10^{-2} \mathrm{~mol}^{-}$ $\mathrm{dm}^{-3} \mathrm{CeCl}_{3}$ 


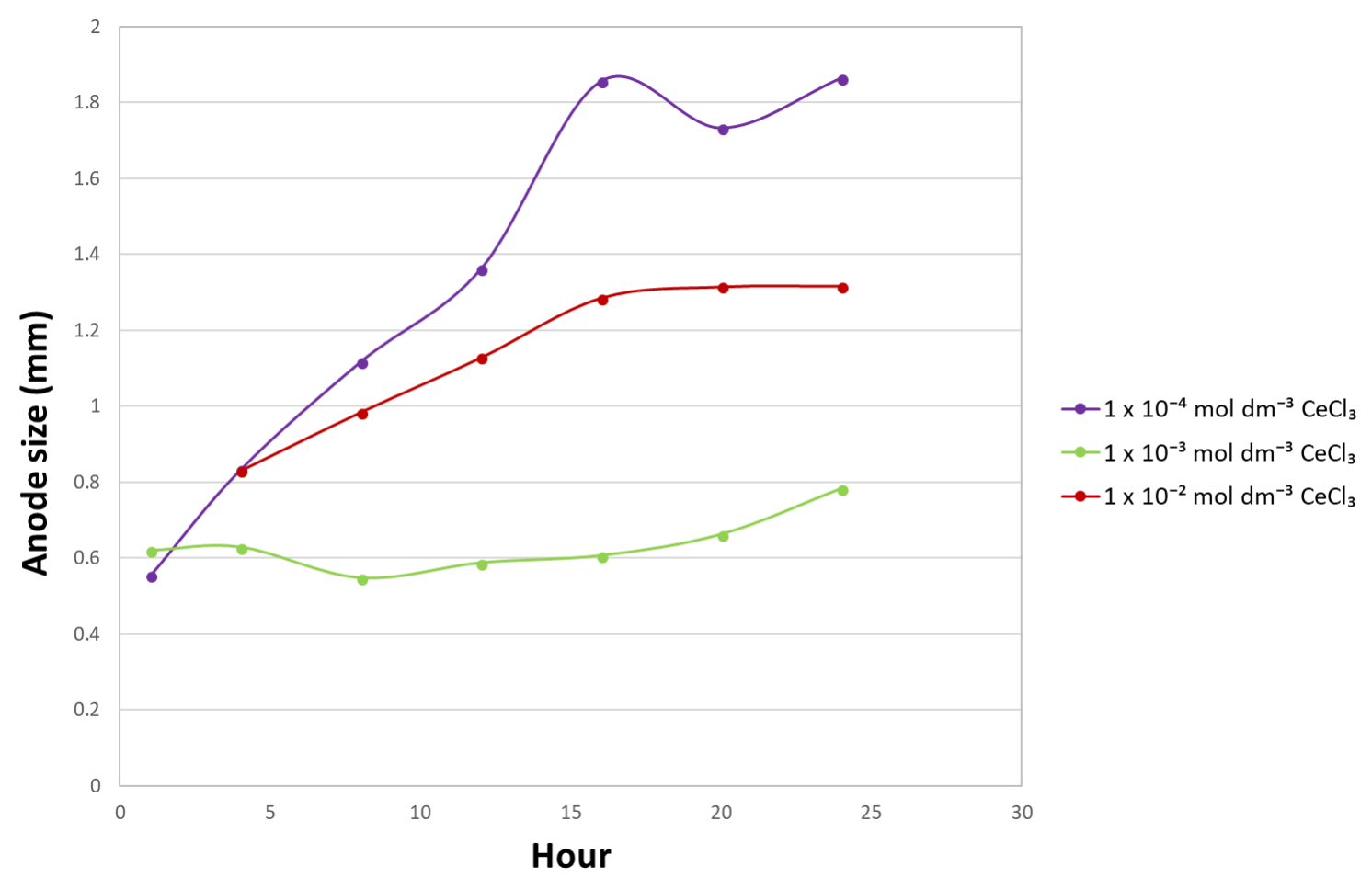

Figure 7.6 Plot of SVET anode size as a function of time for $\mathrm{CeCl}_{3}$

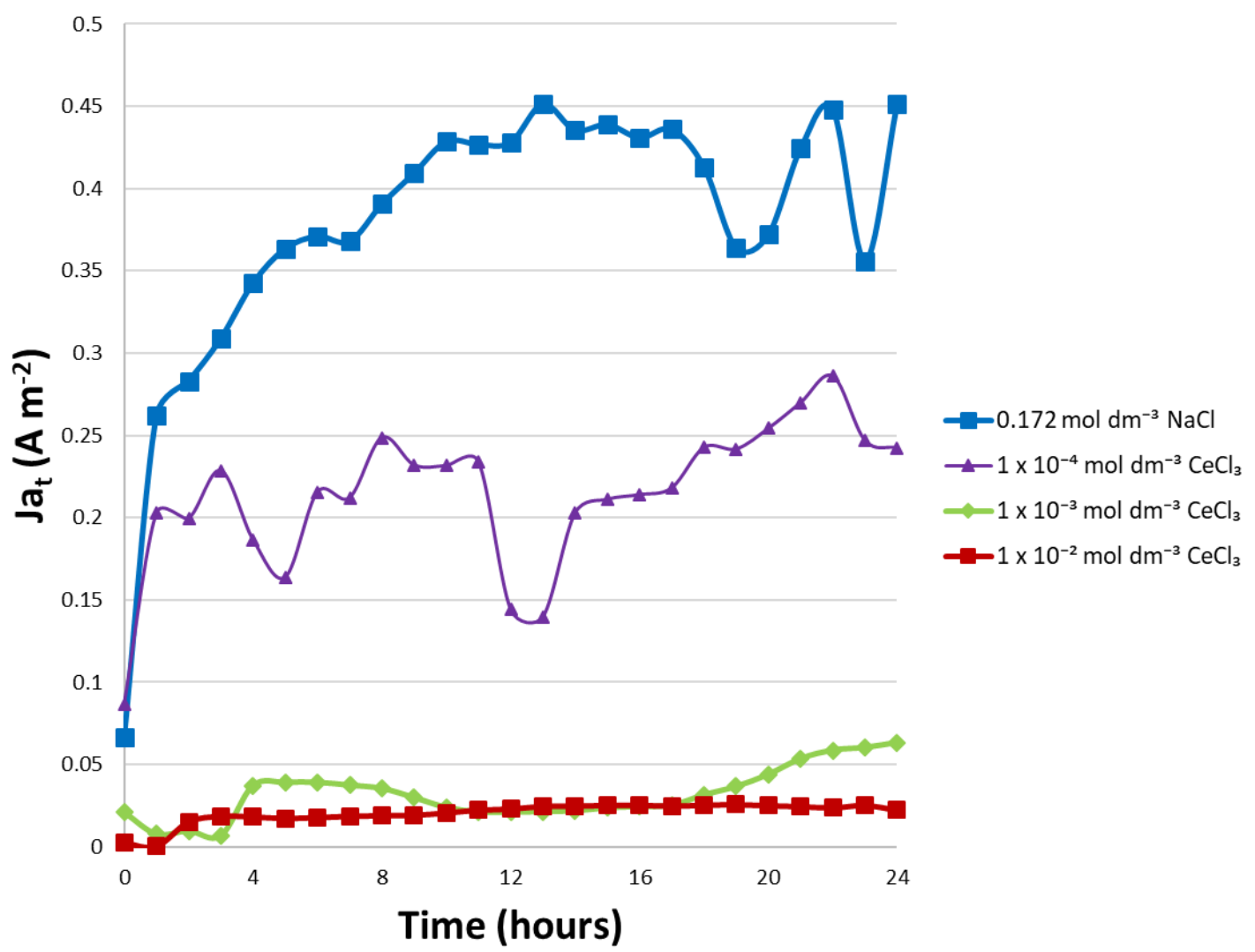

Figure 7.7 The area-averaged anodic-going current density $\left(\mathrm{J} \mathrm{a}_{\mathrm{t}}\right)$ plotted as a function of time for ZMA 2 alloy in $1 \% \mathrm{NaCl}$ pH 7 electrolyte, encompassing the range of $\mathrm{CeCl}_{3}$ inhibitor additions 
7.3.2 Examination of $\mathrm{CeCl}_{3}$ interaction as a corrosion inhibitor on a $\mathrm{ZMA}$ alloy using timelapse optical microscopy

The mechanisms of interaction for $\mathrm{CeCl}_{3}$ on the ZMA 2 alloy have been studied using time-lapse optical microscopy. This was carried out via digital image capture at set two-minute intervals over a period of 50 hours. The inhibitors at the given range of concentrations were dosed into the electrolyte once an anode of sufficient size was established. For the range of concentrations, initial images captured were taken at the onset of the experiment, followed by those captured immediately preceding the addition of $\mathrm{CeCl}_{3}$ inhibitor. The ensuing images were those taken every 60 minutes. Surplus to the still time-lapse images included in Figure 7.8 , the images captured at given intervals were collated into videos for each concentration of inhibitor species. Videos 7.1-7.3 begin from the lowest concentration of inhibitor addition, up to the highest concentration (i.e. Video 7.3 corresponds to $1 \% \mathrm{NaCl}$ containing $1 \times 10^{-2} \mathrm{~mol} \mathrm{dm}^{-3} \mathrm{CeCl}_{3}$ ).

Figure 7.8 A evidenced the rapid formation of several anodes on the surface of the alloy from the experiment onset, notably within the eutectic. Once an hour had elapsed, Figure 7.8 B clearly demonstrated the evolution of these anodic features, as seen by their growth and agglomeration. A corrosion product was also highly visible within the same figure. In the hours proceeding, the progression of these anodic features was palpable, accompanied by the advancing front of corrosion product depositing on the surface. Notably, two distinct regions of corrosion product intersected, stemming from the definite anodic regions of the sample surface. This corrosion product was seen to develop concurrently with the evolution and growth of the anodic sites. A film deposition continuing adjacent to the expected corrosion product was noticeable in Figure 7.8 C-D, potentially originating from the introduction of the cerium salt inhibitor.

Considering Figure 7.8 E-H, the initial image showed little activity present on the surface of the ZMA 2 alloy. After the first hour of immersion, immediately prior to the addition of $\mathrm{CeCl}_{3}$ at $1 \times 10^{-3} \mathrm{~mol} \mathrm{dm}^{-3}$, both a distinct anodic feature and corrosion product formation was present. The anode displayed a steady progression into Figure $7.8 \mathrm{G}$ attacking the eutectic regions, despite the addition of $\mathrm{CeCl}_{3}$; the corrosion product continued to develop in tandem, advancing at a given distance from the anode. However, Figure $7.8 \mathrm{H}$ demonstrated a reduced development of this predominant anodic feature within the timescale. There was an observable growth of this anode, but appeared somewhat restricted in comparison with the growth rate of the previous hours. At the boundary of the corrosion product away from the anodic feature, the presence of cathodic activity was apparent; distributed across the exposed alloy surface was 
again a surface precipitation seemingly divergent to the conventional corrosion product. This was indicative of the deposition of a surface film resulting from the $\mathrm{CeCl}_{3}$ inhibitor addition.

The images captured for the highest concentration of $\mathrm{CeCl}_{3}$ inhibitor, Figure $7.8 \mathrm{I}-\mathrm{L}$, demonstrated a restraint in anodic growth upon inhibitor introduction once again. However, the extent of this restraint was more apparent than the other experiments. Upon dosing the electrolyte with $\mathrm{CeCl}_{3}$, a discernible layer formed over the surface of the cathodic region surplus to the existing corrosion product. This echoes the notion of the corrosion inhibitor playing a role in deposition of a surface film. This is very much suggestive that the surface film was acting to limit the growth of the anode, and is the mechanism in which the $\mathrm{CeCl}_{3}$ addition inhibits corrosion. Little change was observed between Figure 7.8 K-L in terms of growth of the focal anode. An enrichment of the surface film over the cathodic region was apparent, seeming to add to its existing layer with time. The development or accumulation of this surface film was increasingly noticeable parallel to the increase in inhibitor concentration levels throughout the series of time-lapse images and accompanying videos. This fosters the belief that the $\mathrm{CeCl}_{3}$ was actively hindering the growth of anodic features during corrosion events via the formation of a surface film at cathodic sites. 

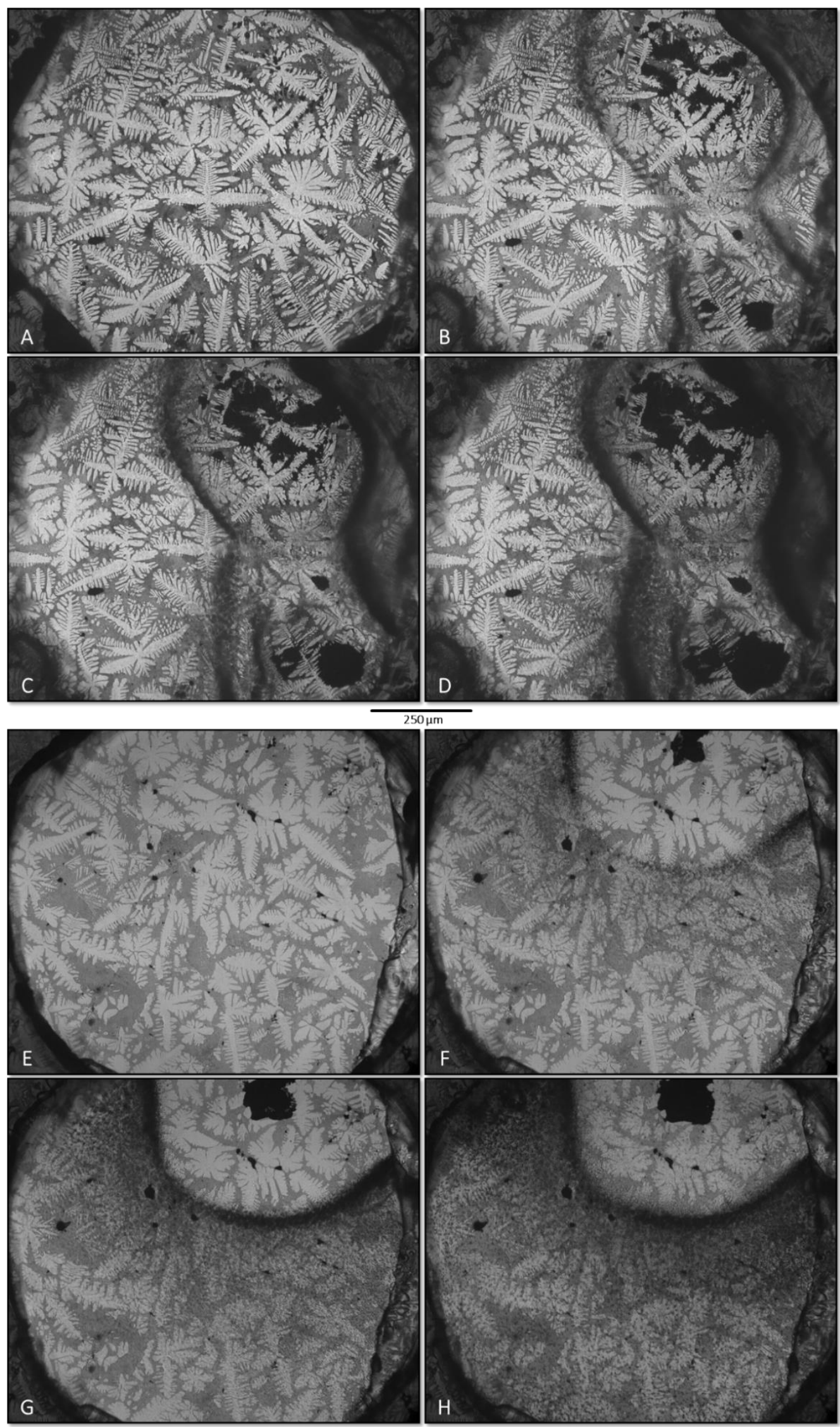

Figure 7.8 Time-lapse microscopy images taken at hourly intervals in a baseline $1 \% \mathrm{NaCl}$ electrolyte, including: (A-D) $1 \times 10^{-4} \mathrm{~mol} \mathrm{dm}^{-3} \mathrm{CeCl}_{3}$, (E-H) $1 \times 10^{-3} \mathrm{~mol} \mathrm{dm}^{-3} \mathrm{CeCl}_{3}$, (I-L) $1 \times 10^{-2} \mathrm{~mol}^{-3}$ $\mathrm{dm}^{-3} \mathrm{CeCl}_{3}$ 


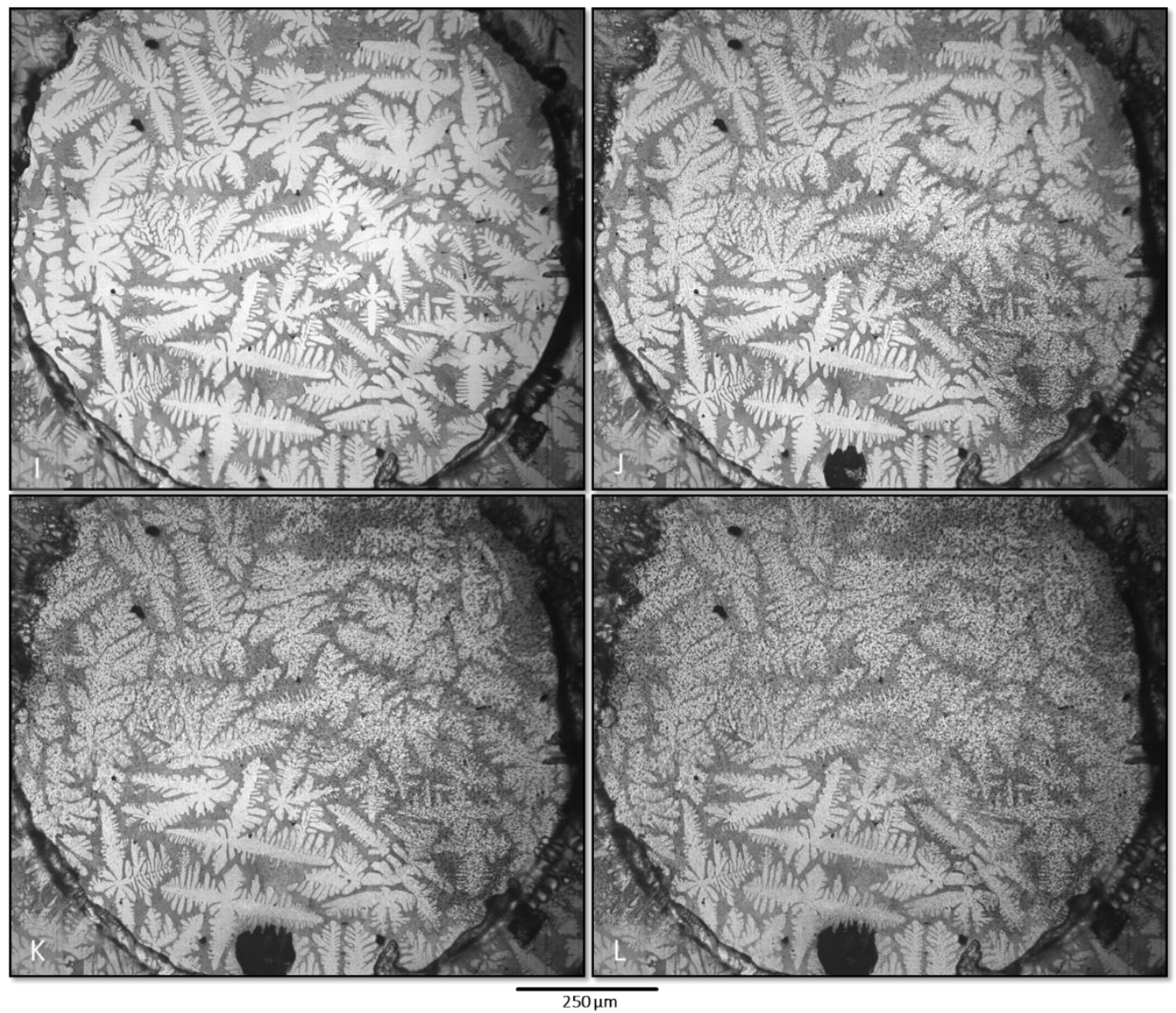

Figure 7.8 Continued

Using the time-lapse images, the corroded area for a given unit of time was plotted in Figure 7.9, based upon the area of anodic attack. An assumption was made that excessive vertical pitting did not occur during these experiments, and instead that corrosion progressed across the sample surface due to the two-dimensional restrictions of this technique. Using Photoshop CS6, the anodic areas were selected and then measured in $\mathrm{mm}^{2}$. The measurements were performed through calibration on a pixel-to-distance basis, using an image of known area with identical resolution and magnification. This analysis was performed for time-lapse images at hourly intervals and over a 30-hour total time-frame. The lateral progression of the corroded area against time plotted in Figure 7.9 demonstrated apparent differences across the spectrum of inhibitor concentrations. The rate of anodic development was markedly increased for the lowest concentration of inhibitor comparative to the remainder. The escalation of the corroded area for this lower concentration was sustained throughout, until the surface was seen to be entirely consumed by corrosive attack at Hour 28. The two higher concentrations of inhibitor species highlighted a significantly reduced extent of anodic evolution; the rate of this diminished in the hours following the introduction of the $\mathrm{CeCl}_{3}$ succeeding hour 1 . The rate of corroded area for $1 \times 10^{-2} \mathrm{~mol} \mathrm{dm}^{-3} \mathrm{CeCl}_{3}$ was seen to fall following hour 2, and this same drop- 
off in growth rate was noted for $1 \times 10^{-3} \mathrm{~mol} \mathrm{dm}^{-3} \mathrm{CeCl}_{3}$ an hour later. The observable difference was likely associated to the ability of the inhibitor to form a cerium-rich film on the surface. The rate of corroded area for the highest concentration inhibitor was evidently stable for the 30hour time-frame, displaying a small increase overall. The $1 \times 10^{-3} \mathrm{~mol} \mathrm{dm}^{-3} \mathrm{CeCl}_{3}$ plot followed a similar stable trend for several hours, yet of a higher magnitude. The line was seen to taper away increasingly so with time. This suggests that increasing quantities of inhibitor have a longer-lasting corrosion inhibition effect and the ability to react more readily to form a surface film. Additionally, the total corroded area over the 30-hour time-frame was measured using image analysis, as plotted in Figure 7.10. This indicated the overall measure of anodic attack on the surface, and the plot showed a distinct ranking between the inhibitor concentration variation. The plot illustrated that increasing concentrations of $\mathrm{CeCl}_{3}$ acted to reduce the extent of corrosive attack on the surface, tying in firmly with SVET-derived metal loss results given in Figure 7.1.

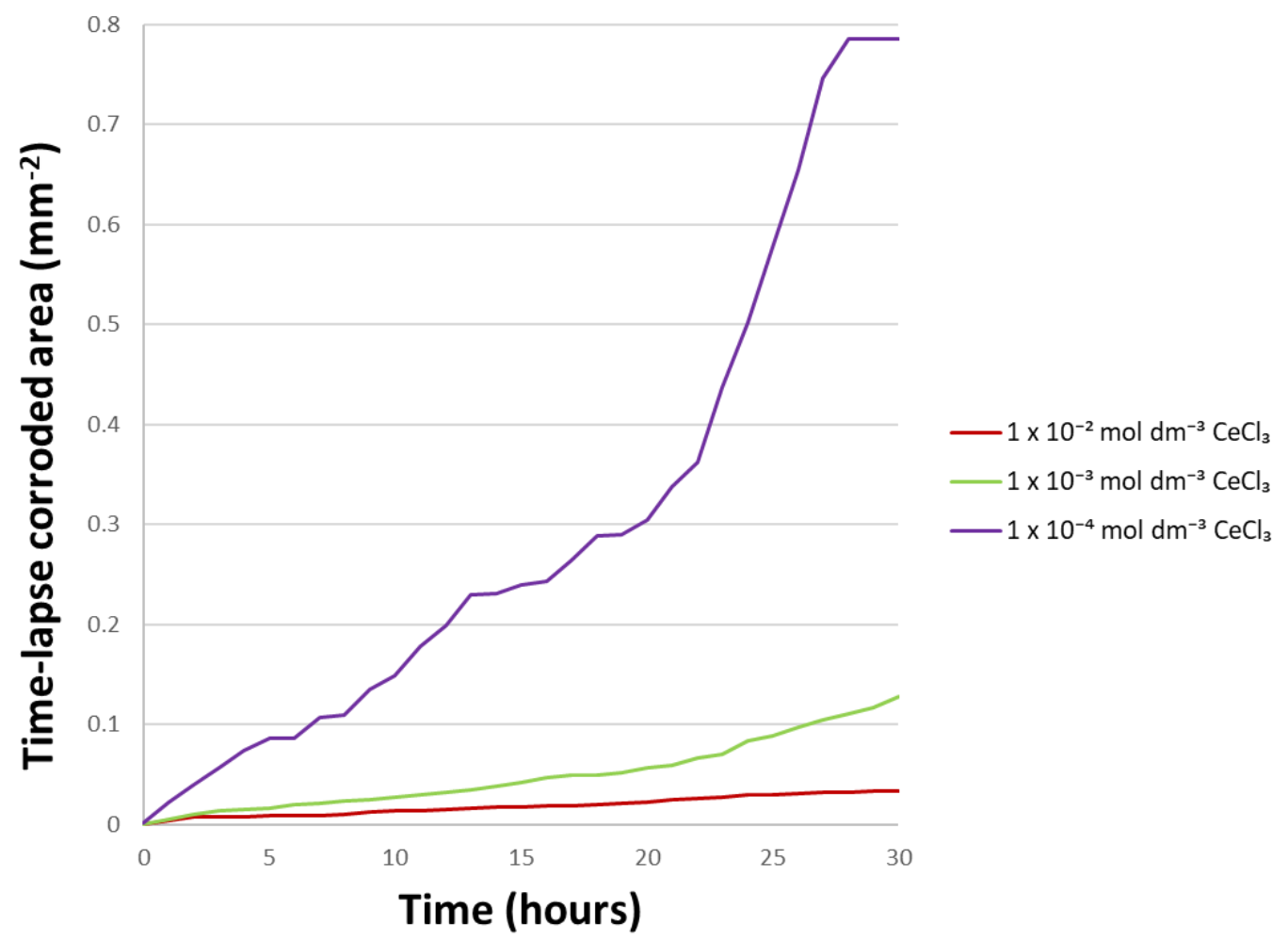

Figure 7.9 Size of corroded area versus time for each inhibitor concentration in $1 \% \mathrm{NaCl}$ electrolyte 


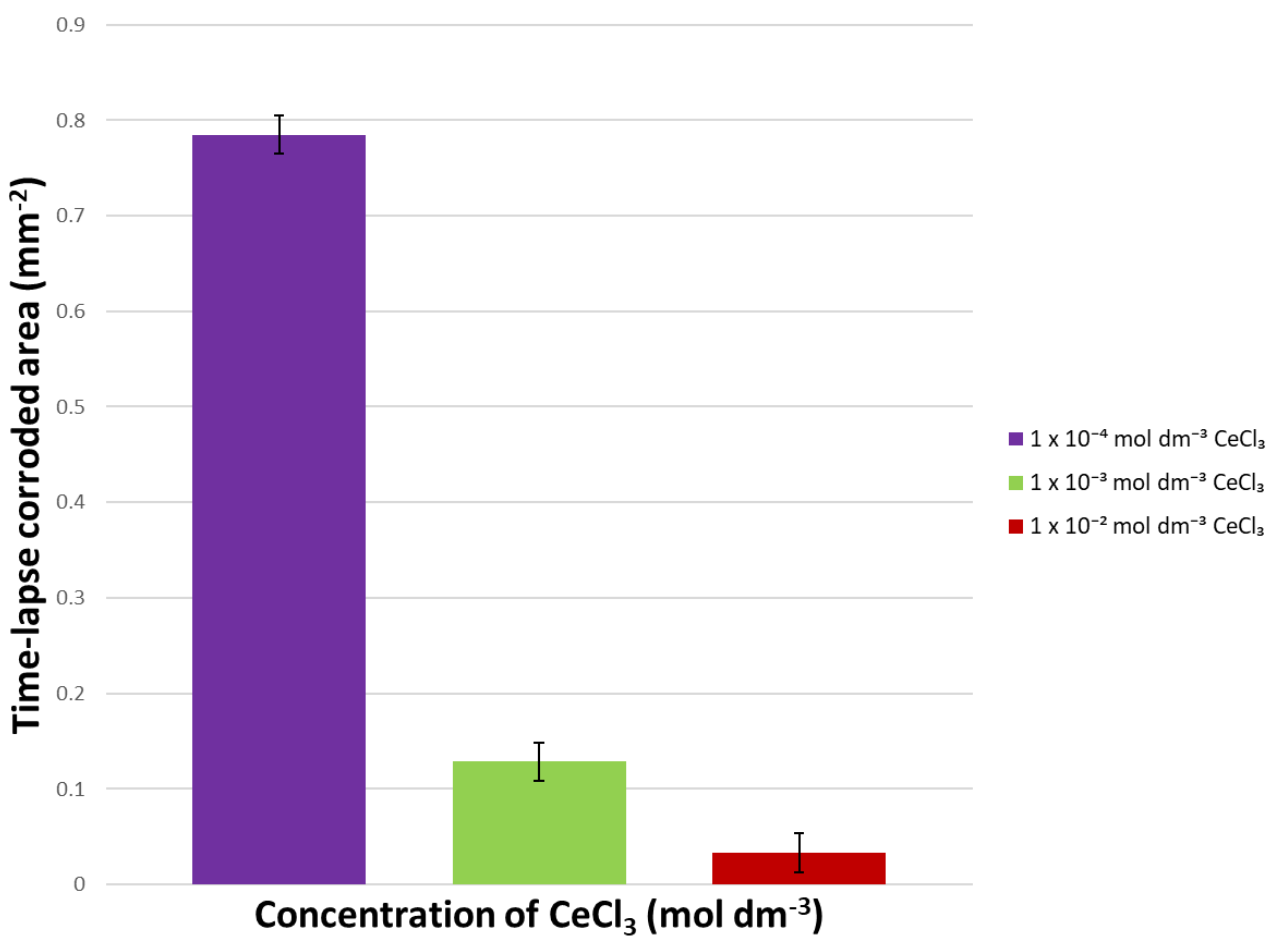

Figure 7.10 Total corroded area for each inhibitor concentration in $1 \% \mathrm{NaCl}$ electrolyte

7.3.3 Open-circuit potential and potentiodynamic polarisation of $\mathrm{CeCl}_{3}$ additions

To further appreciate the mechanisms underlying the action of $\mathrm{CeCl}_{3}$ additions for a ZMA 2 alloy, other electrochemical testing was performed. During open-circuit potential testing, samples were allowed to freely corrode for 30 minutes and were conducted over the range of inhibitor concentrations $\left(1 \times 10^{-4}\right.$ up to $\left.1 \times 10^{-2} \mathrm{~mol} \mathrm{dm}^{-3} \mathrm{CeCl}_{3}\right)$, including an uninhibited control solution of $1 \% \mathrm{w} / \mathrm{v} \mathrm{NaCl}$. Potentiodynamic polarisation was also performed for the same electrolyte range. Polarisation testing is traditionally swept cathodically and anodically, with respect to the open-circuit of the system, as a single experiment; that was not the case for this. The experiment was performed in a different manner to ascertain the impact of $\mathrm{CeCl}_{3}$ on corrosion behaviour, fundamentally arising from any variations perceived during separate sweeping routines. This regimen constituted isolating the anodic and cathodic regions relative to the open-circuit. A ZMA 2 sample was immersed in solution and was allowed to corrode at its' open-circuit for 30 minutes, establishing a stable value. The sample was then polarised slightly positive of its open-circuit, sweeping in a cathodic-going fashion from approximately 1.05 V to -1.4 V versus SCE. Likewise, for the anodic-going procedure, a fresh sample was allowed to rest at its open-circuit potential for 30 minutes. Subsequently, this was polarised marginally negative to the open-circuit of the system, ranging from approximately $-1.06 \mathrm{~V}$ to $0 \mathrm{~V}$ versus SCE. This isolated sweeping protocol was utilised for each different electrolyte as a 
means of more accurately determining the mechanism or action of the $\mathrm{CeCl}_{3}$ on the ZMA system, comparative to a single polarisation sweep.

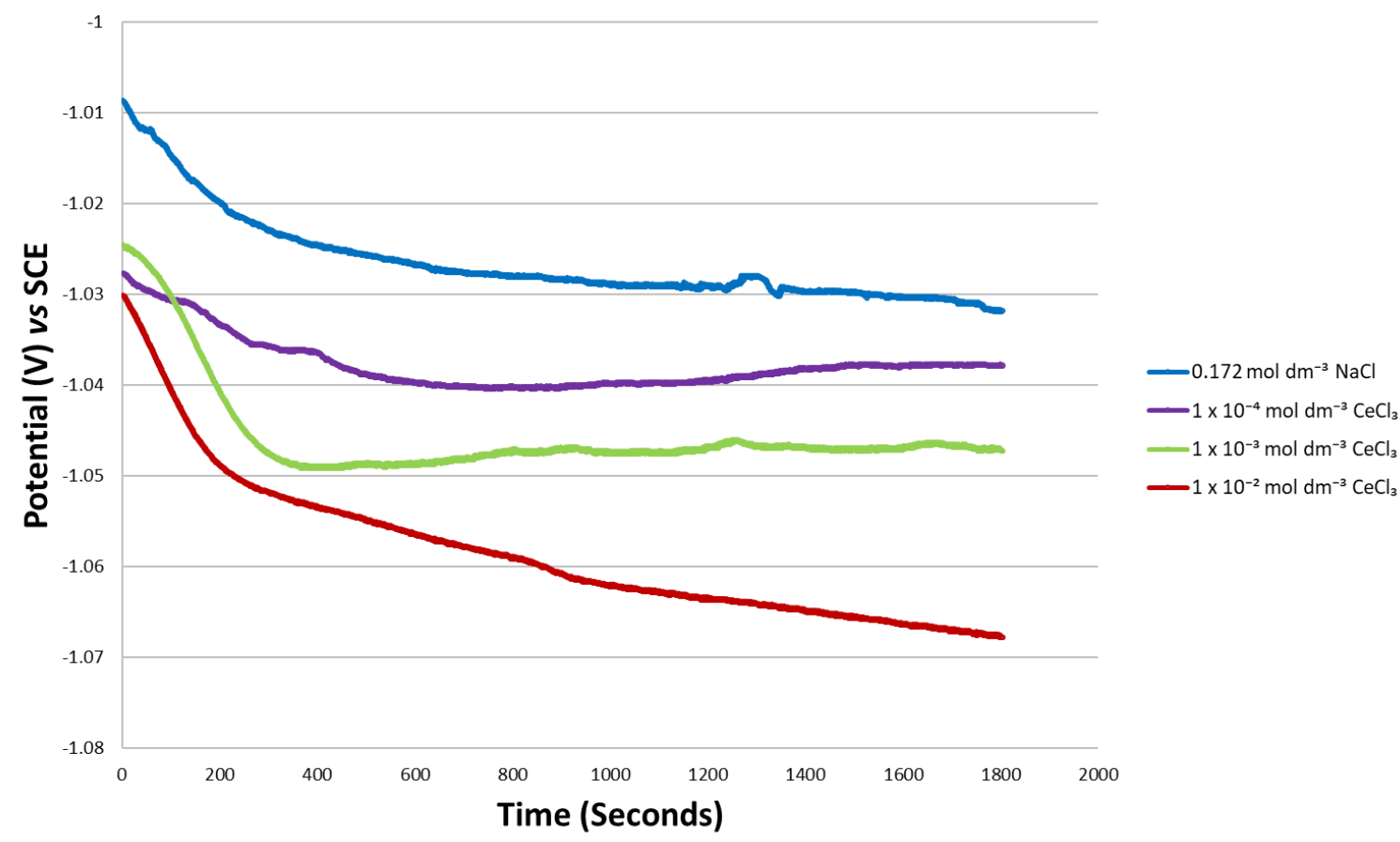

Figure 7.11 Plot of open-circuit potential testing (versus SCE) over the range of dosed inhibitor concentrations in $1 \% \mathrm{NaCl} \mathrm{pH} 7$ baseline electrolyte

As shown in Figure 7.11, all measured potentials were seen to diminish from the point of experiment onset, achieving a degree of stability in terms of measurement. The overall trend of the results indeed demonstrated that the uninhibited sample possessed the largest value of free corrosion potential $\left(\mathrm{E}_{\text {corr }}\right)$. The results then revealed a reduction in $\mathrm{E}_{\text {corr }}$ upon introduction of $\mathrm{CeCl}_{3}$ at the lowest concentration. Accordingly, an inverse relationship between inhibitor concentration and $\mathrm{E}_{\text {corr }}$ became apparent, as an increase in concentration of inhibitor species depressed the measured $E_{\text {corr }}$. The Evans diagram for cathodic protection (see Figure 1.9) can be used to better understand the action of corrosion inhibitor species in a corrosive electrolyte; this predicts that an inhibitor acting to suppress the cathodic reaction would decrease not only the cathodic current density, but the overall $\mathrm{E}_{\text {corr }}$ value as well. This explains the drop in measured free corrosion potential with progressive increase in inhibitor addition, and adds weight to the concept of $\mathrm{CeCl}_{3}$ acting as a cathodic inhibitor for this ZMA system. 


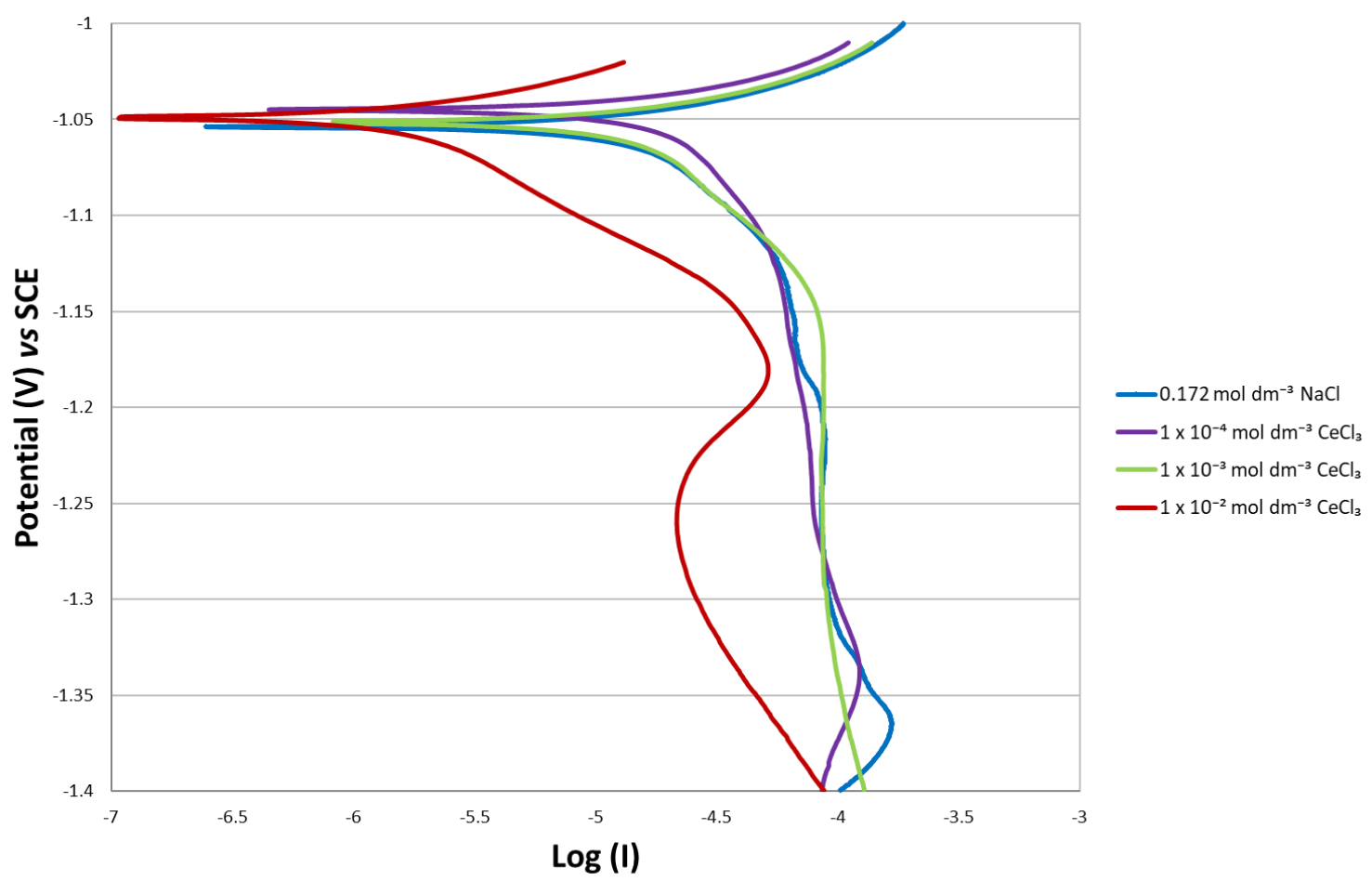

Figure 7.12 Plot of cathodic-going polarisation curves for the range of dosed inhibitor concentrations in $1 \% \mathrm{NaCl}$ pH 7 baseline electrolyte

In Figure 7.12, the polarisation curves isolated for the cathodic-going potentiodynamic sweep, relative to the open-circuit are displayed. The uninhibited sample and that of the lowest two inhibitor additions, $1 \times 10^{-4} \mathrm{~mol} \mathrm{dm}^{-3}$ and $1 \times 10^{-3} \mathrm{CeCl}_{3}$, followed particularly similar trends over the duration of the testing. However, the highest concentration of $\mathrm{CeCl}_{3}$ offered obvious differences from the polarisation curve. Whilst the other curves demonstrated a moderately sharp increase in log (I) values from their open-circuit, this effect was dampened for the higher concentration. This was indicative of a reduction in mass transport of oxygen from bulk solution to the surface of the sample, up to a peak $\log (\mathrm{I})$ value at $-1.18 \mathrm{~V}$ (vS SCE). From this peak value, $\log (\mathrm{I})$ diminished readily until $-1.26 \mathrm{~V}$, slowly returning to values observed for the other completed curves. The observed fall in measured current once again suggested a restriction in oxygen availability to the alloy surface, but to a much greater extent. The implication is that $\mathrm{CeCl}_{3}$ reacted to form a surface film, impeding the ability of oxygen to reach the ZMA surface, and limiting the rate of the cathodic reaction. 


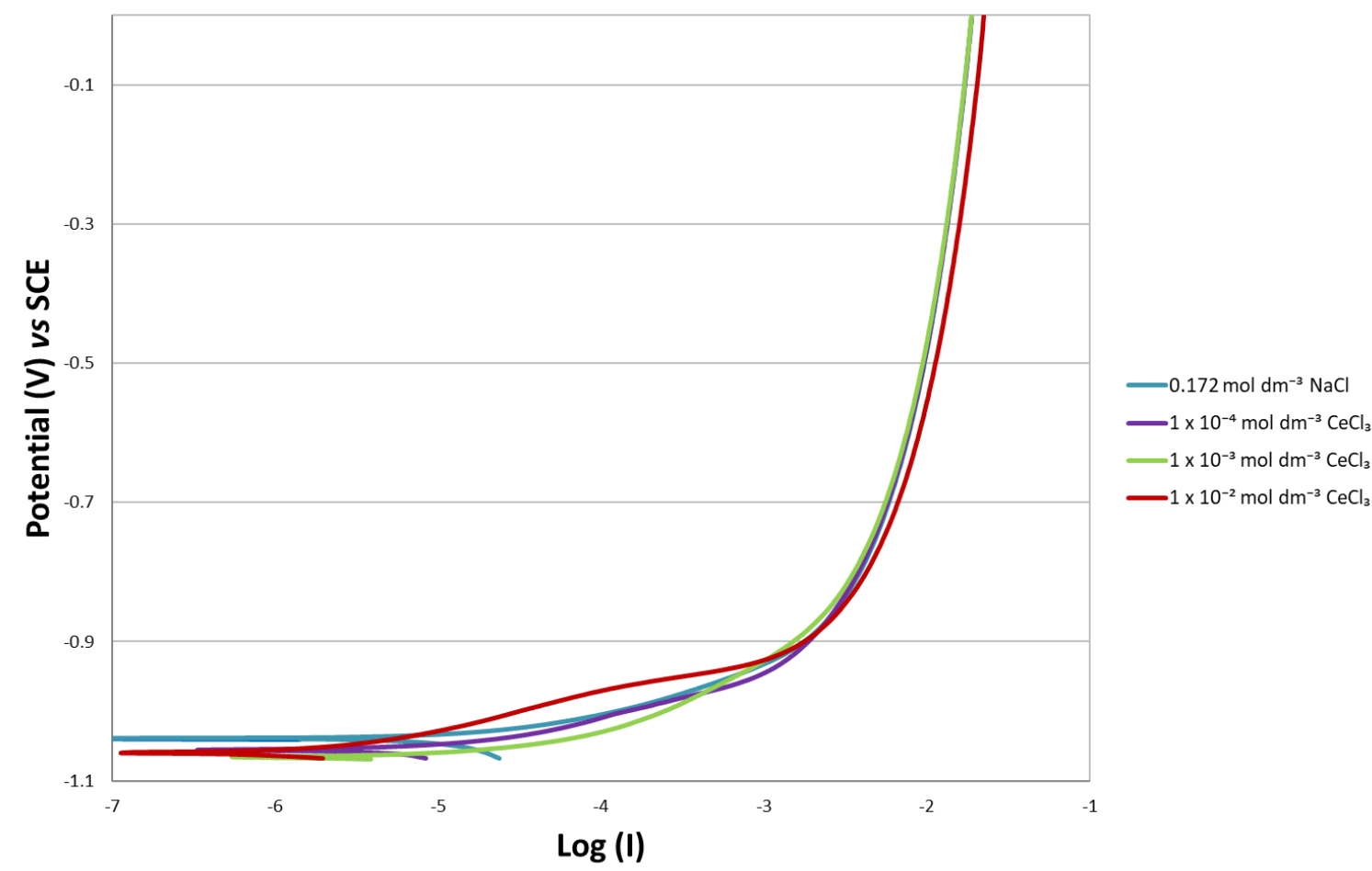

Figure 7.13 Plot of anodic-going polarisation curves for the range of dosed inhibitor concentrations in $1 \% \mathrm{NaCl} \mathrm{pH} 7$ baseline electrolyte

The anodic-going potentiodynamic polarisation curves given in Figure 7.13 elaborate on the mechanism of action for the $\mathrm{CeCl}_{3}$ inhibitor. The two lowest inhibitor concentrations and uninhibited sample all displayed curves resembling one another; whilst the highest concentration of $\mathrm{CeCl}_{3}$ addition was generally very similar to the entire set of polarisation curves, a subtle difference was observable. The highest concentration curve showed minor evidence of passivation between approximately $-1.02 \mathrm{~V}$ up to $-0.94 \mathrm{~V}$ vs SCE. Previous work concluded that the presence of sufficient cerium ions in solution interfered with the mechanism governing dissolution of zinc. This same study subsequently proposed that a cerium complex adsorbate can act as a nucleation site for a cerium hydroxide/oxide [43]. This may explain the subtle passivation perceivable during the anodic-going polarisation test for the highest inhibitor concentration.

Table 7.2 Measured metal loss values via SVET and Tafel-extrapolation

\begin{tabular}{|c|c|c|}
\hline $\begin{array}{c}\mathrm{CeCl}_{3} \text { concentration } \\
\left(\mathrm{mol} \mathrm{dm}^{-3}\right)\end{array}$ & $\begin{array}{c}\text { SVET metal } \\
\text { loss }\left(\mathrm{g} \mathrm{m}^{-2}\right)\end{array}$ & $\begin{array}{c}\text { Tafel-derived metal } \\
\text { loss }\left(\mathrm{g} \mathrm{m}^{-2}\right)\end{array}$ \\
\hline 0 & 12.20 & 6.09 \\
\hline $1 \times 10^{-4}$ & 7.41 & 5.59 \\
\hline $1 \times 10^{-2}$ & 0.84 & 0.76 \\
\hline
\end{tabular}

The Tafel-extrapolated values given in Table 7.2 indicated a reduction in metal loss as the concentration of inhibitor dosed in solution was increased. The values for the inhibitor- 
containing experiments were more comparable to those found by SVET. However, the control experiment free of inhibitor showed largely different values between the two techniques, with Tafel extrapolation underestimating the metal loss values on the whole. Some degree of confidence can be realised from this data, which illustrated an equal trend between both experimental techniques.

\subsubsection{Study of inhibitor surface deposition using SEM and EDS}

Following corrosion testing using the SVET, a sample used for the $1 \times 10^{-2} \mathrm{~mol} \mathrm{dm}^{-3} \mathrm{CeCl}_{3}$ was kept aside upon completion of the experiment. The sample was examined post-corrosion to determine the composition of any surface films or products following the 24-hour SVET test. This was performed using a Hitachi TM3000 Tabletop Scanning Electron Microscope and Bruker Quantax70 EDS system.
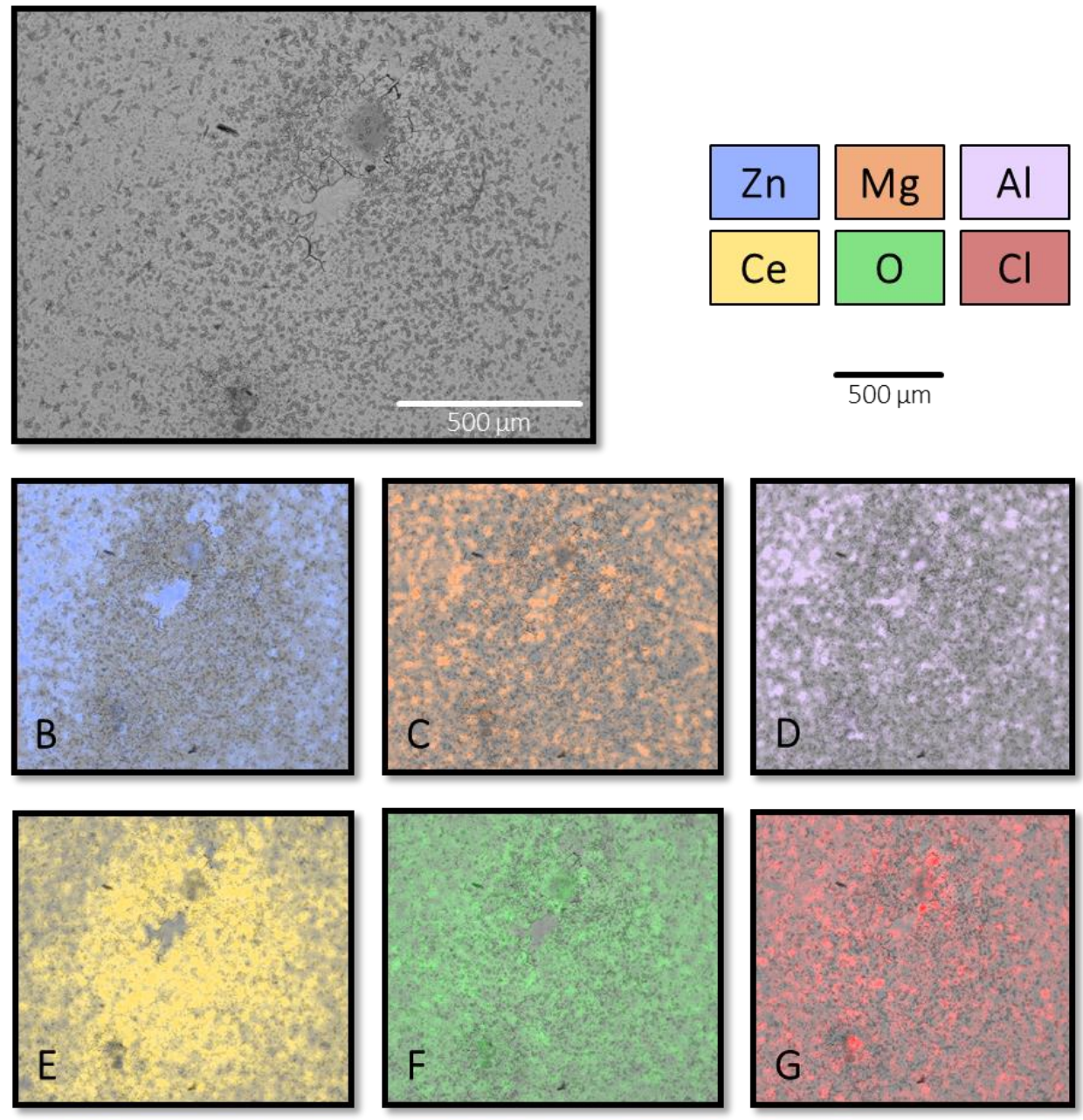

Figure 7.14 (A-G) SEM and EDS analysis of a ZMA sample following immersion in $1 \times 10^{-2} \mathrm{~mol} \mathrm{dm}^{-3}$

$\mathrm{CeCl}_{3}$

Figure 7.14 A was taken as a representative image from a central position of the corroded sample area. Figure 7.14 B-G go on to show the elemental analysis obtained from EDS mapping 
of this surface. The constitutional alloying elements of zinc, magnesium and aluminium were all present on the surface, including a small nodule towards the centre primarily made up of these three elements. The elemental maps went on to show that zinc was unsurprisingly apparent on the surface, but to a lesser degree on the interior of the image. However, this interior area was shown to be distinctly rich in cerium, indicating that the inhibitor certainly acted to deposit a Ce-based film on the ZMA surface under immersion conditions. The presence of oxygen was also clear, covering the majority of the sample and indicating the presence of oxide or hydroxide-based corrosion product. Previous works considering cerium salts have concluded that cerium-based oxides or hydroxides are precipitated on the surface under accelerated corrosion conditions [42]-[46]. As such, for the purposes of this body of work it is assumed that the composition is of a cerium oxide or hydroxide.

\subsubsection{Validation of SVET-measured metal loss using gravimetric analysis}

Any SVET-derived metal loss values are considered semi-quantitative due to the limitations inherent to the technique and the associated data manipulation. Consequently, gravimetric analysis was performed for select samples as a means of evaluating the accuracy of the metal loss quantities drawn from SVET experimentation. This was conducted according to ISO 8407, and a full explanation of the procedure and associated sample preparation is explained in 2.7. Samples underwent gravimetric testing using two different inhibitor concentrations dosed into the baseline $1 \% \mathrm{NaCl} \mathrm{pH} 7$ electrolyte, consisting of $1 \times 10^{-2} \mathrm{~mol} \mathrm{dm}^{-3}$ and $1 \times 10^{-4} \mathrm{~mol} \mathrm{dm}^{-3}$ $\mathrm{CeCl}_{3}$. The control sample data has also been included to confirm the effectiveness of the inhibitor. All samples were prepared in a similar fashion to those for SVET experimentation, undergoing a polishing, etching, and masking procedure. A pre-corroded weight was recorded prior to masking with PTFE tape. The ZMA samples were then immersed in their inhibitorcontaining electrolyte for 24 hours, to mimic the time-frame employed during SVET experimentation. Upon sample removal at the end of the allocated immersion time, samples underwent chemical cleaning cycles using a saturated glycine solution in an ultrasonic bath. Following each cleaning cycle, samples were cleaned, dried, and their weight recorded. This process was repeated until corrosion products were removed from the surface.

Considering Table 7.3, for the highest concentration of $\mathrm{CeCl}_{3}$, SVET-derived results indicated an underestimation in the weight lost from the sample relative to the gravimetric value. Contrarily, the lower concentration inhibitor addition revealed a larger quantity of metal loss according to SVET than recorded gravimetrically. Some reasons for the observed discrepancies may relate to the limitations of the SVET technique itself, and assumptions made 
during data interpretation. One factor leading to an underestimation of metal loss may be the fact that the SVET records a vector component of current density, rather than the direct surface current density. Another consideration is the resolution of SVET, whereby anodic and cathodic sites separated by a distance less than the scan height are undetectable and will not be recorded. Additionally, it has been assumed that the rate of corrosion is identical between the hourly scan intervals, which may not be the case. These may go on to explain the underestimated value from SVET. However, this does not explain a SVET-recorded overestimation in value for the lowest concentration inhibitor addition. Instead, this may simply allude to the variation possible from one experiment to another, and the limitation of the apparatus utilised for gravimetric analysis. The magnitude of weight loss from samples during this form of testing is minute, and as such is highly sensitive. SVET-derived results were recorded in situ and are based upon ionic current flux emanating from a surface corrosion site, whereas gravimetric analysis is a post-corrosion bulk measurement of the entire sample. For gravimetric analysis, an analytical balance was utilised, capable of $0.1 \mathrm{mg}$ readability $( \pm 0.2 \mathrm{mg}$ linearity) and given the scale of metal loss from the samples, readings were subject to a margin of error. Furthermore, for consistency of results, recorded values were scaled to $\mathrm{g} \mathrm{m}^{-2}$ and as such, any degree of error would be amplified.

Table 7.3 Comparison of measured metal loss values via gravimetric and SVET analysis

\begin{tabular}{|c|c|c|c|c|}
\hline $\begin{array}{c}\mathrm{CeCl}_{3} \\
\text { concentration (mol } \\
\mathrm{dm}^{-3} \text { ) }\end{array}$ & $\begin{array}{c}\text { SVET metal } \\
\text { loss }\left(\mathrm{g} \mathrm{m}^{-2}\right)\end{array}$ & $\begin{array}{c}\text { Gravimetric metal } \\
\text { loss }\left(\mathrm{g} \mathrm{m}^{-2}\right)\end{array}$ & $\begin{array}{c}\text { SVET-measured } \\
\text { inhibition } \\
\text { efficiency }\end{array}$ & $\begin{array}{c}\text { Gravimetric- } \\
\text { measured } \\
\text { inhibition efficiency }\end{array}$ \\
\hline 0 & 12.20 & 10.95 & $\mathrm{~N} / \mathrm{A}$ & $\mathrm{N} / \mathrm{A}$ \\
\hline $1 \times 10^{-4}$ & 7.41 & 6.97 & $39 \%$ & $36 \%$ \\
\hline $1 \times 10^{-2}$ & 0.84 & 1.49 & $93 \%$ & $86 \%$ \\
\hline
\end{tabular}

Whilst discrepancies were present between the differing forms of experiment, they were by no means outside the realms of error on this scale. Moreover, the area of surface exposed to the electrolyte differed between SVET and gravimetric analysis, which may have influenced the extent of corrosion under these conditions. Overall, the comparison of the differing experiments was a good indication of the accuracy of SVET-derived results and considered sufficient to verify data obtained by this technique. The comparison exhibited similar inhibition efficiencies and established that the inhibitor was effective even at the lowest concentration.

It is worth noting that the Tafel-extrapolated metal loss data presented in Table 7.4 was less comparable, as indicated by the SVET and gravimetric data sets. This was most extreme for the control experiment containing no inhibitor, although the remaining data was more akin 
overall. The reasons for the stronger deviations noted by Tafel extrapolation may be due to the fact that potentiodynamic polarisation is a perturbing technique, or simply that this form of data acquisition is less accurate for localised corrosion [96].

Table 7.4 Summary of measured metal loss values

\begin{tabular}{|c|c|c|c|}
\hline $\begin{array}{c}\mathrm{CeCl}_{3} \text { concentration } \\
\left(\mathrm{mol} \mathrm{dm}^{-3}\right)\end{array}$ & $\begin{array}{c}\text { SVET metal } \\
\text { loss }\left(\mathrm{g} \mathrm{m}^{-2}\right)\end{array}$ & $\begin{array}{c}\text { Gravimetric metal } \\
\text { loss }\left(\mathrm{g} \mathrm{m}^{-2}\right)\end{array}$ & $\begin{array}{c}\text { Tafel-derived metal } \\
\text { loss }\left(\mathrm{g} \mathrm{m}^{-2}\right)\end{array}$ \\
\hline 0 & 12.20 & 10.95 & 6.09 \\
\hline $1 \times 10^{-4}$ & 7.41 & 6.97 & 5.59 \\
\hline $1 \times 10^{-2}$ & 0.84 & 1.49 & 0.76 \\
\hline
\end{tabular}

7.3.6 Exploration of the corrosion inhibition behaviour of $\mathrm{CeCl}_{3}$ in a $\mathrm{ZMA}$ alloy

Previous work considering cerium salts and the effect on a range of metallic substrates concluded that cerium acts to inhibit the cathodic reaction via the formation of an insoluble film [40]-[43]. Considering the studies completed for this section of work, a similar conclusion can be drawn. The data attained from SVET demonstrated that introduction of the $\mathrm{CeCl}_{3}$ into solution altered the mechanism of corrosion on the surface of the alloy, seen in Figure 7.3-7.5. The evolution of anodic activity was shown to decrease with additions of $\mathrm{CeCl}_{3}$ inhibitor, with focal anodes displaying a marked restriction in growth in general. The SVET anode size analysis revealed that progressive growth of the anode was not necessarily more restricted for the highest inhibitor concentration; instead, the intensity and number of anodic sites was diminished compared to the intermediate concentration. Thereby explaining the differences. The SVET-measured metal loss results exhibited palpable increases in corrosion performance via a fall in recorded metal loss values, parallel to increasing concentrations of rare earth inhibitor. This alone indicated the ability of $\mathrm{CeCl}_{3}$ to inhibit corrosion as concentration was increased, within the ZMA system.

The area-averaged anodic current density values found in Figure 7.7 highlighted differences over the duration of the SVET experiments. Despite the similarity in measured metal loss values for the two highest concentrations, the plot suggested that towards the latter stages of the experiment, the $1 \times 10^{-3} \mathrm{~mol} \mathrm{dm}^{-3}$ addition diminished in performance. However, the $1 \mathrm{x}$ $10^{-2} \mathrm{~mol} \mathrm{dm}^{-3}$ addition was much more consistent and revealed an element of stability up until the end of the test. Consequently, it is entirely possible that whilst the strict magnitude of performance may be limited for this inhibitor system, the higher quantity of addition may have had a longer lasting inhibition effect beyond the scope of the 24-hour test. This was echoed by Figure 7.5 , where the current density map clearly displayed a lessened degree of activity 
overall. The results obtained from time-lapse microscopy reaffirmed such observations and illustrated a distinct ranking.

Figure 7.8 displayed a restriction of anodic growth for those with increasing quantities of inhibitor present once again, clarifying that higher inhibitor concentrations offered improved corrosion resistance levels. The introduction of the inhibitor following the onset of corrosion allowed the conventional corrosion product to be more easily distinguished from inhibitor interaction. The images demonstrated that the presence of $\mathrm{CeCl}_{3}$ additions encouraged the formation of a surface film dissimilar to the conventional corrosion product, precipitating in the cathodic regions of the exposed surface. This cerium-rich film was seen to cover a significant proportion of the surface area available in Figure $7.8 \mathrm{I}-\mathrm{L}$, masking only the region that would be considered an area of cathodic activity. There appeared to be no direct deposition immediate to the anodic site, however the anode growth was certainly restricted comparative to lower inhibitor concentrations and the uninhibited experiment considered from Chapter 3 . It is reasonable to deduce that the anodic restriction was a consequence of deposition of the cerium-based film at the cathodic region, limiting the ability of oxygen to adsorb on the surface of the metal-to-electrolyte interface. It is well-established within galvanising alloys that the primary cathodic mechanism is that of oxygen reduction, which therefore, justifies this effect. Accordingly, this limitation of mass transport to the surface would act to throttle the anodic reaction, becoming a rate-determining step for the overall corrosion kinetics. Investigations into cerium salts have shown that in cathodic regions where oxygen reduction predominates, it is understood that cerium oxides and hydroxides are formed [43]-[46]. The valence of these cerium-rich layers, namely cerium(III) or cerium(IV), have been reported to vary across the literature [41], and it has been suggested that this is partially dependant on the time immersed in the cerium salt [44], [45]. For this reason, it has been theorised that these formed layers are of a cerium oxide/hydroxide composition. The formation of cerium oxide could occur per Equation 7.1, and in the case of trivalent cerium, production of an insoluble cerium hydroxide could occur via Equation 7.2. Upon close scrutiny of Figure 7.8 K-L, the cerium film was seen to possess a darkened layer amongst an array of precipitated particles. The abundance of these particles was observed to increase as the experiment progressed, forming an increasingly compact layer. This was seen to a lesser degree for the $1 \times 10^{-3} \mathrm{~mol} \mathrm{dm}^{-3} \mathrm{CeCl}_{3}$ images, the morphology of which was less compact for the equivalent time interval; very little of the cerium film was perceivable for the lowest concentration within the given image intervals. 


$$
\mathrm{Ce}+\mathrm{O}_{2} \rightarrow \mathrm{CeO}_{2}
$$

$$
2 \mathrm{Ce}^{3+}+6 \mathrm{OH}^{-} \rightarrow 2 \mathrm{Ce}(\mathrm{OH})_{3}
$$

The fact that similar levels of corrosion performance were recognised via SVET for the two higher inhibitor concentrations again suggested a factor of time-dependency; this was based on the SVET-derived values of $\mathrm{Ja}_{\mathrm{t}}$ offering more stable values over time associated with the highest concentration of $\mathrm{CeCl}_{3}$. The analysis of the time-lapse images endorsed this understanding, indicating that the highest concentration of $\mathrm{CeCl}_{3}$ provided a greater degree of inhibition over a longer time-frame. This was made clear by the discrepancy in performance between the two highest inhibitor quantities, which was more pronounced in Figure 7.9-7.10 than that from SVET-recorded metal loss values. The image analysis time-frame was beyond that of the SVET experiment, and only towards the latter stages of the time-lapse sequences was an obvious fall in performance revealed for $1 \times 10^{-3} \mathrm{~mol} \mathrm{dm}^{-3} \mathrm{CeCl}_{3}$. This perhaps highlights that the presence of increasing quantities of $\mathrm{CeCl}_{3}$ facilitates the process of inhibition, but that the cerium can only form oxide and/or hydroxide layers at a limited rate. If the overall corrosion process is slowed, then products from the cathodic reaction (i.e. hydroxide ions) will be less abundant. A lower availability of such products would limit the ability of the inhibitor to react and form cerium-rich layers, allowing the inhibition to continue, yet at a reduced rate. Alternatively, increased cerium concentrations may yield a prompter reaction, permitting the formation of cerium layers more readily and allowing a more continuous barrier effect. This would make the route for dissolved oxygen more problematic, acting as a chokehold on the anodic reaction for prolonged periods of time. This is in agreement with the literature; Arenas et al. concluded that higher concentrations of $\mathrm{CeCl}_{3}$ additions formed a less porous cerium-based corrosion product, and that lower concentrations allowed galvanised steel to form zinc-based corrosion products more rapidly, providing a less adherent surface for cerium deposition [43]. The extended inhibition effect realised via time-lapse analysis, combined with the increase in observable compactness of the cerium surface products for $1 \times 10^{-2} \mathrm{~mol} \mathrm{dm}^{-3} \mathrm{CeCl}_{3}$ ties in with the understanding that higher concentrations of this inhibitor yield less porous layers. Based on the above evidence, progressively larger additions of $\mathrm{CeCl}_{3}$ may extend the length of time that an inhibition effect is seen.

It can be realised from the results that both SVET and time-lapse microscopy arrived at the same ranking of performance for the inhibitor quantities between the techniques. The SVET 
can detect current flux associated with the corrosion reaction on the sample surface, whereas time-lapse microscopy is limited to two-dimensional observations. Ordinarily, a consequence of this would be that time-lapse microscopy would underestimate the results seen comparative to SVET. Instead, the difference in magnitude of corrosion activity was more pronounced for timelapse microscopy, which was likely attributable to the extended time-frame of those experiments over that of SVET.

The SEM-EDS maps of a ZMA sample following 24 hours immersion in the $1 \% \mathrm{w} / \mathrm{v} \mathrm{NaCl}$ including $1 \times 10^{-2} \mathrm{~mol} \mathrm{dm}^{-3} \mathrm{CeCl}_{3}$ clearly highlighted the presence of cerium-rich compounds on the sample surface. The maps indicated a significant proportion of cerium and oxygen, suggesting the existence of cerium oxide/hydroxide, confirming their presence following introduction of the inhibitor species into the corrosive electrolyte. Regarding the most immediate corrosion products to form upon the onset of corrosion, it is understood that for magnesium-containing zinc alloys, the presence of Mg-rich phases causes rapid formation of magnesium-based corrosion products [72], [78], [79], [113], such as that given in Equation 3.5. A consequence of this is a $\mathrm{pH}$ buffering effect from magnesium carbonate compounds, deterring the formation of zinc oxide [72]. As to whether magnesium-based corrosion products provided an adherent surface for deposition of cerium-based films however, is difficult to comment on without additional characterisation. Nevertheless, the presence of these magnesium compounds are proposed to facilitate the formation of insoluble zinc-based corrosion products [47], thereby potentially affecting the ability of cerium to form stable, adherent precipitates. Whilst the $\mathrm{pH}$ buffering effect brought about by magnesium may initially hinder formation of cerium oxide/hydroxide, the ensuing surface corrosion products may prove beneficial for adhesion overall.

The potentiostat-based open-circuit potential and potentiodynamic polarisation work featured in 7.3.3 extended support to $\mathrm{CeCl}_{3}$ acting as a cathodic inhibitor. The relative opencircuit potentials ( $E_{\text {corr }}$ ) clearly depicted an inverse relationship, falling as concentrations of the inhibitor species were increased. This trend corroborates with the Evans diagram for cathodic protection, for increasing quantities of inhibitor. The polarisation curves went on to reveal a pronounced effect to the cathodic branch, extending from approximately $-1.05 \mathrm{~V}$ to $-1.4 \mathrm{~V} v \mathrm{~s}$ $\mathrm{SCE}$, for the highest $\mathrm{CeCl}_{3}$ concentration. This effect took the form of diminished measured log (I) values. The anodic-going polarisation curves contrasted with the cathodic-going curves showed minimal effects, clarifying the impact on cathodic activity for $\mathrm{CeCl}_{3}$. However minimal the observed changes in the anodic-going curves were, a small degree of passivation was seen for the highest inhibitor addition. Research into the effect of $\mathrm{CeCl}_{3}$ on commercially galvanised 
steel theorised that initial chloride surface adsorption was disrupted by the adsorption of cerium ions, which go on to act as nucleation sites for the development of cerium layers [43]. A similar effect may have been evident here, marginally slowing the rate of the anodic process at the highest concentration of cerium salt.

\subsection{Conclusions}

The techniques utilised for this section of work have proved to be highly useful in terms of elucidating an understanding of the effect of $\mathrm{CeCl}_{3}$ on a ZMA system. A distinct trend emerged demonstrating that for concentrations up to $1 \times 10^{-2} \mathrm{~mol} \mathrm{dm}^{-3} \mathrm{CeCl}_{3}$, the addition offered significant reductions to the extent of corrosion. The series of results were in firm agreement, unanimously finding the same ranking of performance across the range of dosed additions; an inverse relationship between inhibitor concentration and extent of corrosive attack was shown, where the highest quantity of inhibitor species provided the lowest degree of corrosion. The development of anodic features was shown to be restricted with increasing concentrations of $\mathrm{CeCl}_{3}$, as given by both time-lapse microscopy and SVET current density map data sets. SVET metal loss data revealed only mild performance improvements associated with the increase from $1 \times 10^{-3} \mathrm{~mol} \mathrm{dm}^{-3} \mathrm{CeCl}_{3}$ to $1 \times 10^{-2} \mathrm{~mol} \mathrm{dm}^{-3} \mathrm{CeCl}_{3}$. However, SVET-derived area-averaged anodic current density plots suggested a greater degree of stability with respect to time for the highest concentration, and this conflict was further clarified. The time-lapse experiments and corresponding data conducted over a longer experimental time-frame indicated a larger performance gap between the two greatest inhibitor additions. The time-lapse corroded area analysis indicated a fall in performance for $1 \times 10^{-3} \mathrm{~mol} \mathrm{dm}^{-3} \mathrm{CeCl}_{3}$ toward the latter stages of the experiment; this was not evident for the highest inhibitor addition counterpart. Accordingly, the extended time-frame highlighted the ability of the higher $\mathrm{CeCl}_{3}$ concentration to offer continued corrosion inhibition effects. The estimated values of metal loss obtained via SVET were contrasted to that generated from gravimetric analysis, and this comparison was considered sufficient to substantiate SVET-related data. However, the Tafel-extrapolated metal loss data was less accurate, especially so for the control solution free of inhibitor addition. Otherwise, this was closer to the SVET-derived values, and overall showing an underestimation in metal loss comparative to both SVET and gravimetric results.

The inhibition effects corresponding to the cerium salt additions were a result of the deposition of a surface film. Time-lapse microscopy images demonstrated that for higher $\mathrm{CeCl}_{3}$ concentrations surface layers were deposited dissimilar to the conventional corrosion products. Although, these layers were not as evident in time-lapse images for $1 \times 10^{-4} \mathrm{~mol} \mathrm{dm}^{-3} \mathrm{CeCl}_{3}$. The 
precipitates consisted of an array of particles amongst surface films, the compactness of which increased in parallel with inhibitor addition quantity. The surface deposition was notably visible away from the anodic site and instead, formed within regions of cathodic activity. The restrained growth of anodic sites was associated with the formation of the insoluble surface films. It was deduced from EDS maps that following 24-hour immersion in $1 \% \mathrm{NaCl}$ electrolyte with $1 \times 10^{-2} \mathrm{~mol} \mathrm{dm}^{-3} \mathrm{CeCl}_{3}$, cerium-based compounds were indeed rich on the surface. Consistent with the literature, it has been assumed that such surface films were of a cerium oxide/hydroxide composition. The mechanism by which the surface films inhibited corrosion was via moderation of oxygen adsorption at the metal-to-electrolyte interface. The presence of these layers at the cathodic site imposed a restriction on the overall cathodic reaction, which is primarily $\mathrm{O}_{2}$ reduction for zinc-based alloys. The inflicted rate limitation consequently impacted on the rate of anodic reaction also. This in turn explained the marked restriction in visible growth of anodic features for increasing concentrations of inhibitor. Furthermore, the rate of cerium-rich compound deposition was seen to increase in accordance with a rise in quantity of inhibitor species. From this, it was understood that the ability to form a more continuous barrier layer would improve corrosion resistance levels by strangling the kinetics of ensuing corrosion events.

Open-circuit potential testing revealed a fall in measured free corrosion potential for increasing $\mathrm{CeCl}_{3}$ inhibitor additions. The outcome aligned with the Evans diagram for cathodic protection, adding weight to the argument that $\mathrm{CeCl}_{3}$ acts as a cathodic inhibitor. With respect to the potentiodynamic polarisation curves, isolated sweeping protocols were utilised and demonstrated that the action of $\mathrm{CeCl}_{3}$ as an inhibitor was primarily seated in its ability to affect the cathodic reaction. At $1 \times 10^{-2} \mathrm{~mol} \mathrm{dm}^{-3} \mathrm{CeCl}_{3}$, the cathodic sweep exhibited a notable depression in log (I). The anodic counterpart showed only a subtle impact, potentially a result of cerium ions interfering with adsorption of chloride ions at the sample surface. However, little effect was drawn from the remaining inhibitor concentration variations for both sweeping protocols. 


\section{Chapter 8 Conclusions and further work}

\subsection{Conclusions}

The work presented within this thesis considering zinc-based galvanising alloys has reached a range of conclusions, as outlined below.

ZMA alloys have been studied according to three different magnesium and aluminium additions, to ascertain their impact on microstructure and corrosion behaviour. As studied using optical-light microscopy and SEM-EDS, the microstructures of these alloys were observed to constitute a primary zinc-rich dendrite phase amongst a eutectic matrix, and smaller quantities of an aluminium-rich dendritic phase. The eutectic phases were made up of a coarse binary eutectic, and a fine ternary eutectic; the binary lamellar eutectic was composed of $\mathrm{Zn}$ and $M g Z n_{2}$ phases, and the ternary, $Z n, M g Z n_{2}$, and Al. The microstructural composition was not exactly as predicted by the ternary phase diagram, which predicted the formation of $\mathrm{Mg}_{2} \mathrm{Zn}_{11}$ rather than $\operatorname{MgZn}_{2}$. However, this $M g Z n_{2}$ phase can be understood to form consequent of the non-equilibrium cooling conditions. A change in the alloy content demonstrated an increase in eutectic phase volume fraction with a corresponding drop in primary zinc-rich dendrites, resulting from increasing $\mathrm{Mg}$ and $\mathrm{Al}$ content; this also led to a reduction in size of individual primary $\mathrm{Zn}$ dendrites.

The SVET revealed that corrosion resistance levels improved in tandem with increasing quantities of both $\mathrm{Mg}$ and $\mathrm{Al}$ alloying additions, in the studied aqueous $1 \% \mathrm{w} / \mathrm{v}(0.172 \mathrm{M}) \mathrm{NaCl}$ electrolyte. This was observed through a reduced extent of anodic evolution and number of anodic sites present, alongside a fall in SVET-measured metal loss values. Time-lapse optical microscopy demonstrated that corrosion initiated in the eutectic phases, specifically attacking the $M g Z n_{2}$ phase as clarified by SEM. Beyond eutectic attack in the first instance, corrosion was seen to progress on primary $\mathrm{Zn}$ phases and the remaining microstructure. The time-lapse microscopy data and subsequent analysis showed full agreement with SVET-based corrosion ranking, indicating improved corrosion resistance in conjunction with greater alloying quantities. The observed improvements were associated with preferential attack of Mg-rich phases and the formation of beneficial corrosion products, understood to reduce corrosion kinetics and hinder zincite formation. The presence of favourable protective species likely composed magnesium and zinc-based compounds during initial stages, such as zinc hydroxide and magnesium hydroxide; this was followed by zinc-based compounds in the form of hydrozincite and simonkolleite, and Al-containing layered double hydroxides, ultimately 
providing enhanced corrosion properties. Furthermore, oxide layers of magnesium and aluminium, in comparison with zincite, are understood to be advantageous to corrosion resistance levels; this was, in part, noted to contribute to increased resistance to corrosion as the alloying elements were increased.

A selected ZMA 2 alloy composing Zn-2wt.\% Mg-2wt.\% Al was subsequently studied, to appreciate the effect of $\mathrm{pH}$ on the corrosion properties under immersion conditions. At neutral and alkaline $\mathrm{pH}$ conditions, a characteristic point anode to ring anode evolution was observed by SVET, accompanied by localised anodic attack and ring of corrosion product detected via time-lapse microscopy. At higher conditions of $\mathrm{pH}$, hydroxides and oxides are thought to form, and grant passivity to the alloy surface, as indicated by the discussed Pourbaix diagrams. However, the overall surface states of the alloy predicted by the Pourbaix diagrams were only considered as an indicator, due to the presence of chloride media, and factors such as localised $\mathrm{pH}$ conditions at the alloy surface. At acidic conditions, the ZMA 2 alloy evidenced a shift to a more generalised corrosion mechanism, displaying widespread attack across the exposed surface; this altered corrosion mechanism was also observed by SVET. This was partly attributed to the prevalence of cathodic hydrogen evolution in place of oxygen reduction. The corrosion process was observed to initiate in the eutectic phases for all $\mathrm{pH}$ conditions, however the progression differed between $\mathrm{pH} 3$, and for $\mathrm{pH} 7-10$. The more acidic media revealed that the primary Zn-rich dendrites generally supported cathodic activity amongst distributed anodic attack; cathodic activity for the higher pH electrolytes was more discrete on the surface at some distance from the focal anodic features, and only proceeded to attack more noble phases once of an established size. Open-circuit potential values became increasingly negative for lower $\mathrm{pH}$ conditions, and potentiodynamic polarisation data showed diminishing log (I) values as $\mathrm{pH}$ was increased. The time-lapse microscopy experiments illustrated a more pronounced extent of attack at $\mathrm{pH} 3$ than realised by SVET. This is proposed to be a consequence of the change in corrosion mechanism beyond the resolution of the SVET, and thus, leading to an underestimation in corrosion activity as validated by gravimetric analysis. The experimental data generally conferred improvements in corrosion performance at higher $\mathrm{pH}$ conditions, attributable to the enhanced presence and stability of favourable corrosion products.

A range of corrosion inhibitor additions have been studied at three different concentrations by dosing into a baseline $1 \% \mathrm{w} / \mathrm{v}(0.172 \mathrm{M}) \mathrm{NaCl} \mathrm{pH} 7$ electrolyte. The corrosion inhibitors considered were that of a compound widely utilised in industry, a rare-earth element, and a more novel organic inhibitor, taking the form of sodium phosphate, cerium chloride, and L-tryptophan, respectively. This set of experimentation was performed to ascertain their 
mechanism and impact on the ZMA alloy system, more specifically, on a designated commercial ZMA 2 alloy. Generally, the SVET revealed that anodic formation and extent of evolution was progressively reduced according to increasing concentrations of inhibitor species. However, this was not the case for the lowest concentration of the organic inhibitor, L-tryptophan; this instead exhibited a lessened resistance to corrosion relative to the control experiment according to gravimetric analysis. For the most part, the time-lapse microscopy data agreed with the results obtained via SVET, with the exception being for the lowest L-tryptophan concentration mentioned above. Otherwise, time-lapse investigations revealed restrictions in anodic growth for increasing quantities of the inhibitors, coincident with the formation of surface precipitates. The extent of the precipitate formation and associated restriction in anodic growth was a function of inhibitor species and concentration. The time-lapse microscopy generally revealed that corrosion was initiated in the eutectic phases at early stages of the experiment regardless of introduced inhibitor; the site of precipitation corresponding to the inhibitor was dependent on the species. Inhibition of any particular phase was not apparent from the time-lapse experiments.

In the case of sodium phosphate, metal phosphate precipitates were observed to deposit at sites of anodic activity. These were predicted to form as insoluble tertiary phosphate species, as indicated by solubility calculations and evidence from SEM-EDS results. The potentiodynamic polarisation data demonstrated increasingly positive potentials (vs SCE) and reductions in log (I) for higher concentrations of the sodium phosphate inhibitor. Corresponding to the Evans diagram for anodic inhibition, this effect was proposed via reaction of metal cations with phosphate anions, producing insoluble metal phosphate species proximal to the site of anodic dissolution. In time-lapse microscopy experiments, an excess of phosphate precipitates was observed to form with time, in the form of filament-like species. These were suggested to propagate via a nucleation and growth mechanism, stemming from a supersaturation of metallic ions in solution. A chromate inhibitor benchmark was studied using SVET also, which revealed that the inhibition ability of this compound was greater than sodium phosphate, even at a concentration two orders of magnitude less.

The other studied inhibitors in the form of L-tryptophan and $\mathrm{CeCl}_{3}$, offered different mechanisms of corrosion inhibition to sodium phosphate. For L-tryptophan, the two higher concentrations of inhibitor were observed to restrict anodic growth rates; a film superfluous to the conventional corrosion product was apparent, originating in cathodic regions of the sample surface. This film was observed to form rapidly, accumulating with time and eventually extending minor coverage to the anodic site. The mechanism of inhibition was understood to 
hinder mass transport of oxygen at the sample surface, thereby restricting the growth of anodic features. However, the mechanism of action for the protective film was unclear. The formation of a poorly soluble metal-L-tryptophan complex was entirely possible, as well as an adsorbed film consequent of bulk surface charge. Regardless, precipitation of the film was predominantly in cathodic regions. In the case of $\mathrm{CeCl}_{3}$, surface films dissimilar to the conventional corrosion product were again visible during time-lapse experiments. These precipitated layers consisted of an array of particles amongst surface films, which were noted to become more compact according to increased concentrations. These surface layers were observed to form away from the focal anodic activity, appearing instead in cathodic regions. The growth of anodic sites was visibly restrained according to the formation of these insoluble surface layers; these were considered to be a cerium oxide/hydroxide, and this was indicated by the supporting SEM-EDS data. In the same fashion as for L-tryptophan, this is understood to limit adsorption of oxygen at the metal-to-electrolyte interface; with oxygen reduction understood to prevail on zinc-based alloys, the presence of such surface layers at cathodic regions would restrict the overall cathodic reaction rate, and consequently, the anodic reaction rate. It was noted that the higher concentration of rare-earth inhibitor enabled an increased rate of deposition on the surface, providing a more continuous barrier layer.

Open-circuit potential testing demonstrated a reduction in potential (vS SCE) as concentrations of both L-tryptophan and $\mathrm{CeCl}_{3}$ were increased. These results aligned with the Evans diagram for cathodic protection and contributed to evidence suggesting that these inhibitors acted by means of cathodic inhibition. The $\mathrm{CeCl}_{3}$ inhibitor at the highest concentration demonstrated a clear influence on the cathodic branch during potentiodynamic polarisation. The corresponding anodic branch showed subtle impact, possibly a result of interference of chloride ion adsorption at the sample surface by cerium ions. However, little impact was drawn from the other studied concentrations. The polarisation data for Ltryptophan was discordant with the data gathered under freely corroding conditions; this was attributed to the perturbation associated with the technique, potentially affecting the mechanism of inhibitor action. This was echoed in the Tafel-extrapolated values of metal loss. The summary of metal loss values included in Table 8.1 revealed some discrepancy between the different techniques utilised for L-tryptophan. The associated variation was attributed to the limitations corresponding to the techniques themselves. The metal loss values for $\mathrm{CeCl}_{3}$ were more substantiated between gravimetric and SVET data sets, with Tafel-extrapolation offering the less accurate results; however, the performance ranking was identical between each technique. 
The summary of data for the corrosion inhibitors studied using SVET Figure 8.1 suggested that the highest concentration of species considered were all certainly effective in reducing the extent of corrosion via diminished values of recorded metal loss. The addition of sodium phosphate into solution offered seemingly the greatest benefit of the selected inhibitors. However, the chromate compound utilised as a point of reference indicated an excellent degree of inhibition in comparison, especially considering the largely reduced concentration included. The gravimetric data summary Figure 8.2 clarified the performance ranking of the L-tryptophan and $\mathrm{CeCl}_{3}$ inhibitors, instead revealing that the rare-earth compound outperformed the amino acid inhibitor, contrary to SVET estimation. Aside from the known efficacy of the chromate compound, sodium phosphate was observed to provide the greatest extent of corrosion protection from the corrosion inhibitors studied.

Table 8.1 Summary of quantitative corrosion data for the corrosion inhibitor additions studied

\begin{tabular}{|c|c|c|c|c|}
\hline Inhibitor species & $\begin{array}{c}\text { Concentration } \\
\left(\mathrm{mol} \mathrm{dm}^{-3}\right)\end{array}$ & $\begin{array}{c}\text { SVET metal } \\
\text { loss }\left(\mathrm{g} \mathrm{m}^{-2}\right)\end{array}$ & $\begin{array}{c}\text { Gravimetric metal } \\
\text { loss }\left(\mathrm{g} \mathrm{m}^{-2}\right)\end{array}$ & $\begin{array}{c}\text { Tafel-derived metal } \\
\text { loss }\left(\mathrm{g} \mathrm{m}^{-2}\right)\end{array}$ \\
\hline Sodium dichromate & $1 \times 10^{-4}$ & 0.12 & - & - \\
\hline \multirow{3}{*}{ Sodium phosphate } & 0 & 12.20 & - & 0.78 \\
\cline { 2 - 5 } & $1 \times 10^{-4}$ & 2.54 & - & 0.15 \\
\cline { 2 - 5 } & $1 \times 10^{-2}$ & 0.23 & - & 0.10 \\
\hline \multirow{3}{*}{ L-tryptophan } & 0 & 12.20 & 10.95 & 1.07 \\
\cline { 2 - 5 } & $1 \times 10^{-4}$ & 9.79 & 11.94 & 2.06 \\
\cline { 2 - 5 } & $1 \times 10^{-2}$ & 0.35 & 5.97 & 6.06 \\
\hline \multirow{3}{*}{$\mathrm{CeCl}_{3}$} & 0 & 12.20 & 10.95 & 6.09 \\
\cline { 2 - 5 } & $1 \times 10^{-4}$ & 7.41 & 6.97 & 5.59 \\
\cline { 2 - 5 } & $1 \times 10^{-2}$ & 0.84 & 1.49 & 0.76 \\
\hline
\end{tabular}




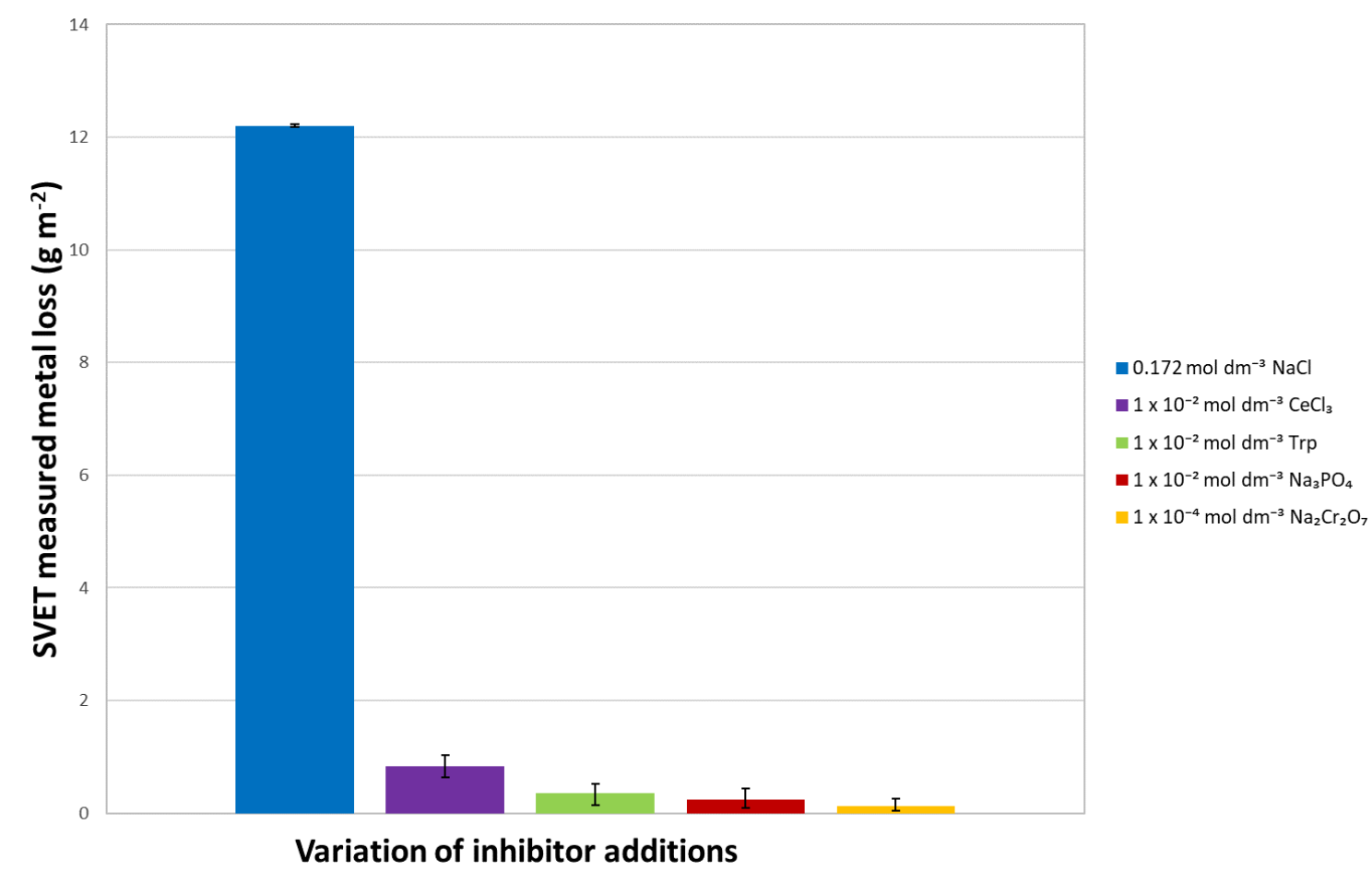

Figure 8.1 Summary of SVET-recorded metal loss values for the studied corrosion inhibitors

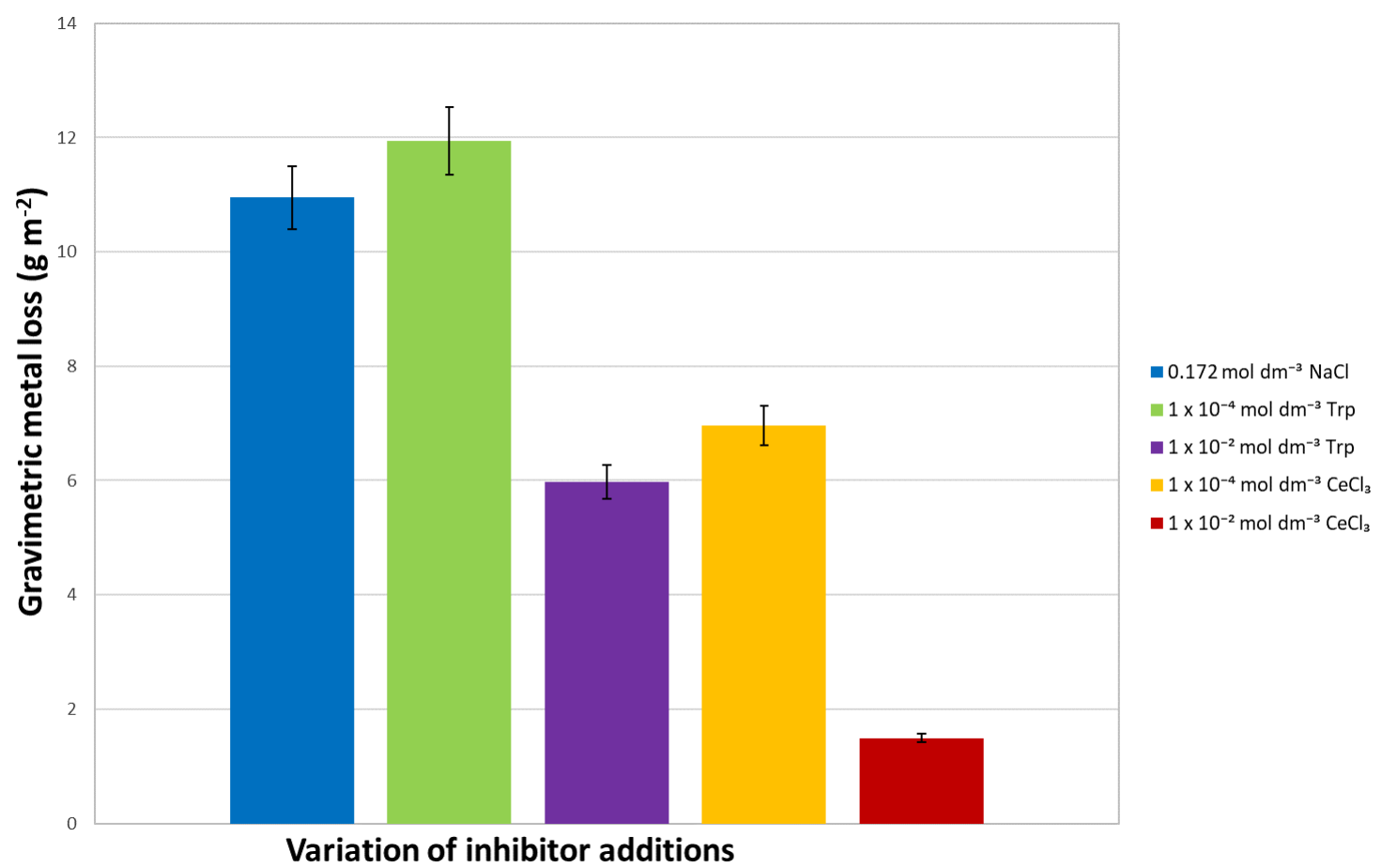

Figure 8.2 Summary of gravimetric analysis for the studied corrosion inhibitors

\subsection{Further work}

To build on the work conducted, other testing may prove useful for further identification or clarification. In terms of the differing ZMA alloy coated steels, additional variants of smaller increments between alloying additions may be of interest; the aim of this would be to assess 
the impact of the additions in an iterative fashion. A closer analysis of the $M g Z n_{2}$ preferential attack using higher magnification studies would be of interest, to further clarify the attack on this phase in the first instance. An analysis of the inter-lamella spacing corresponding to the eutectic could also provide greater insight into the differing corrosion rates associated with the range of ZMA alloys. For the ZMA 2 alloy, a study of additional pH conditions at both greater extremes and more intermediate values than those already considered could be investigated. This may further elucidate whether this ZMA type alloy would prove beneficial in specific applications over other galvanising alloys compositions. Furthermore, the $\mathrm{pH}$ range studies could be extended to the other ZMA alloys examined in Chapter 3, to observe the effect of corrosion performance in different $\mathrm{pH}$ environments according to a change in alloy quantity. Additional characterisation of the inhibitor species pertaining to sodium phosphate, Ltryptophan, and cerium chloride would be of value in order to identify composition of the surface films consequent of such additions. This could be facilitated by techniques such as X-ray photoelectron spectroscopy (XPS) or grazing angle X-ray diffraction (XRD). The interfacial, or polarisation, resistance via electrochemical impedance spectroscopy would also aid in the clarification of the inhibitor interaction with regard to the formation of any passivating films on the metal surface. A more in-depth study of the underlying mechanism for L-tryptophan interaction would also be an avenue for further investigation. 


\section{Chapter 9 References}

\subsection{References}

[1] K. R. Trethewey and J. Chamberlain, Corrosion for students of science and engineering. Longman Scientific \& Technical, 1988.

[2] N. Corlett, L. E. Eiselstein, and N. Budiansky, '2.03 - Crevice Corrosion', in Shreir's Corrosion, B. Cottis, M. Graham, R. Lindsay, S. Lyon, T. Richardson, D. Scantlebury, and H. Stott, Eds. Oxford: Elsevier, 2010, pp. 753-771.

[3] E. Mattsson, Basic corrosion technology for scientists and engineers, the institute of materials. London, 1996.

[4] M. Pourbaix, 'Atlas of electrochemical equilibria in aqueous solutions', M Pourbaix Publ. 1974 NACE 644, 1974.

[5] M. B. Kannan, H. Khakbaz, and A. Yamamoto, 'Understanding the influence of HEPES buffer concentration on the biodegradation of pure magnesium: An electrochemical study', Mater. Chem. Phys., vol. 197, pp. 47-56, Aug. 2017.

[6] 'DoITPoMS - TLP Library The Nernst Equation and Pourbaix Diagrams - Examples of a Pourbaix Diagram'. [Online]. Available: https://www.doitpoms.ac.uk/tlplib/pourbaix/pourbaix_example.php. [Accessed: 03-Aug2017].

[7] R. G. Buchheit, S. B. Mamidipally, P. Schmutz, and H. Guan, 'Active Corrosion Protection in Ce-Modified Hydrotalcite Conversion Coatings', CORROSION, vol. 58, no. 1, pp. 3-14, Jan. 2002.

[8] L. A. Hernandez-Alvarado, L. S. Hernandez, and S. L. Rodriguez-Reyna, 'Evaluation of Corrosion Behavior of Galvanized Steel Treated with Conventional Conversion Coatings and a Chromate-Free Organic Inhibitor', Int. J. Corros., vol. 2012, p. e368130, Nov. 2011.

[9] A. C. Bastos, M. G. Ferreira, and A. M. Simões, 'Corrosion inhibition by chromate and phosphate extracts for iron substrates studied by EIS and SVET', Corros. Sci., vol. 48, no. 6 , pp. 1500-1512, Jun. 2006.

[10] H. S. Isaacs, S. Virtanen, M. P. Ryan, P. Schmuki, and L. J. Oblonsky, 'Incorporation of Cr in the passive film on Fe from chromate solutions', Electrochimica Acta, vol. 47, no. 19, pp. 3127-3130, Jul. 2002.

[11] A. C. Bastos, M. G. S. Ferreira, and A. M. Simões, 'Comparative electrochemical studies of zinc chromate and zinc phosphate as corrosion inhibitors for zinc', Prog. Org. Coat., vol. 52, no. 4, pp. 339-350, Apr. 2005.

[12] M. Svoboda and J. Mleziva, 'Properties of coatings determined by anticorrosive pigments', Prog. Org. Coat., vol. 12, no. 3, pp. 251-297, Jul. 1984.

[13] S. A. Katz, 'The analytical biochemistry of chromium.', Environ. Health Perspect., vol. 92, pp. 13-16, May 1991.

[14] Z. Fang, M. Zhao, H. Zhen, L. Chen, P. Shi, and Z. Huang, 'Genotoxicity of Tri- and Hexavalent Chromium Compounds In Vivo and Their Modes of Action on DNA Damage In Vitro', PLOS ONE, vol. 9, no. 8, p. e103194, Aug. 2014.

[15] K. Salnikow and A. Zhitkovich, 'Genetic and Epigenetic Mechanisms in Metal Carcinogenesis and Cocarcinogenesis: Nickel, Arsenic and Chromium', Chem. Res. Toxicol., vol. 21, no. 1, pp. 28-44, Jan. 2008.

[16] M. Costa and C. B. Klein, 'Toxicity and Carcinogenicity of Chromium Compounds in Humans', Crit. Rev. Toxicol., vol. 36, no. 2, pp. 155-163, Jan. 2006.

[17] R. M. Park, J. F. Bena, L. T. Stayner, R. J. Smith, H. J. Gibb, and P. S. J. Lees, 'Hexavalent Chromium and Lung Cancer in the Chromate Industry: A Quantitative Risk Assessment', Risk Anal., vol. 24, no. 5, pp. 1099-1108, Oct. 2004. 
[18] H. J. Gibb, P. S. J. Lees, P. F. Pinsky, and B. C. Rooney, 'Lung cancer among workers in chromium chemical production', Am. J. Ind. Med., vol. 38, no. 2, pp. 115-126, Aug. 2000.

[19] E. T. Snow, 'A possible role for chromium(III) in genotoxicity.', Environ. Health Perspect., vol. 92, pp. 75-81, May 1991.

[20] I. M. Zin, S. B. Lyon, and V. I. Pokhmurskii, 'Corrosion control of galvanized steel using a phosphate/calcium ion inhibitor mixture', Corros. Sci., vol. 45, no. 4, pp. 777-788, Apr. 2003.

[21] K. Aramaki, 'The inhibition effects of chromate-free, anion inhibitors on corrosion of zinc in aerated $0.5 \mathrm{M} \mathrm{NaCl}^{\prime}$, Corros. Sci., vol. 43, no. 3, pp. 591-604, Mar. 2001.

[22] H. H. Uhlig, D. N. Triadis, and M. Stern, 'Effect of Oxygen, Chlorides, and Calcium lon on Corrosion Inhibition of Iron by Polyphosphates', J. Electrochem. Soc., vol. 102, no. 2, pp. 59-66, Feb. 1955.

[23] Z. Szklarska-Smialowska and J. Mańkowski, 'Cathodic inhibition of the corrosion of mild steel in phosphate, tungstate, arsenate and silicate solutions containing $\mathrm{Ca} 2+\mathrm{ions}$ ', $\mathrm{Br}$. Corros. J., vol. 4, no. 5, pp. 271-275, Sep. 1969.

[24] A. M. Simões, J. Torres, R. Picciochi, and J. C. S. Fernandes, 'Corrosion inhibition at galvanized steel cut edges by phosphate pigments', Electrochimica Acta, vol. 54, no. 15, pp. 3857-3865, Jun. 2009.

[25] P. de Lima-Neto, A. P. de Araújo, W. S. Araújo, and A. N. Correia, 'Study of the anticorrosive behaviour of epoxy binders containing non-toxic inorganic corrosion inhibitor pigments', Prog. Org. Coat., vol. 62, no. 3, pp. 344-350, May 2008.

[26] D.-J. Choi, S.-J. You, and J.-G. Kim, 'Development of an environmentally safe corrosion, scale, and microorganism inhibitor for open recirculating cooling systems', Mater. Sci. Eng. A, vol. 335, no. 1-2, pp. 228-235, Sep. 2002.

[27] S. M. Powell, H. N. McMurray, and D. A. Worsley, 'Use of the Scanning Reference Electrode Technique for the Evaluation of Environmentally Friendly, Nonchromate Corrosion Inhibitors', CORROSION, vol. 55, no. 11, pp. 1040-1051, Nov. 1999.

[28] K. Aramaki, 'An Attempt to Prepare Nonchromate, Self-Healing Protective Films Containing Molybdate on Iron', CORROSION, vol. 55, no. 11, pp. 1020-1030, Nov. 1999.

[29] D.-Q. Zhang, Q.-R. Cai, X.-M. He, L.-X. Gao, and G.-D. Zhou, 'Inhibition effect of some amino acids on copper corrosion in $\mathrm{HCl}$ solution', Mater. Chem. Phys., vol. 112, no. 2, pp. 353-358, Dec. 2008.

[30] D.-Q. Zhang, Q.-R. Cai, X.-M. He, L.-X. Gao, and G. S. Kim, 'Corrosion inhibition and adsorption behavior of methionine on copper in $\mathrm{HCl}$ and synergistic effect of zinc ions', Mater. Chem. Phys., vol. 114, no. 2-3, pp. 612-617, Apr. 2009.

[31] W. A. Badawy, K. M. Ismail, and A. M. Fathi, 'Corrosion control of Cu-Ni alloys in neutral chloride solutions by amino acids', Electrochimica Acta, vol. 51, no. 20, pp. 4182-4189, May 2006.

[32] K. M. Ismail, 'Evaluation of cysteine as environmentally friendly corrosion inhibitor for copper in neutral and acidic chloride solutions', Electrochimica Acta, vol. 52, no. 28, pp. 7811-7819, Nov. 2007.

[33] L. Bazzi, S. Kertit, and M. Hamdani, 'Some Organic Compounds as Inhibitors for the Corrosion of Aluminum Alloy 6063 in Deaerated Carbonate Solution', CORROSION, vol. 51, no. 11, pp. 811-817, Nov. 1995.

[34] G. Bereket and A. Yurt, 'The inhibition effect of amino acids and hydroxy carboxylic acids on pitting corrosion of aluminum alloy $7075^{\prime}$, Corros. Sci., vol. 43, no. 6, pp. 1179-1195, Jun. 2001.

[35] H. Ashassi-Sorkhabi, M. R. Majidi, and K. Seyyedi, 'Investigation of inhibition effect of some amino acids against steel corrosion in $\mathrm{HCl}$ solution', Appl. Surf. Sci., vol. 225, no. 1-4, pp. 176-185, Mar. 2004. 
[36] M. A. Amin, S. S. Abd El Rehim, and H. T. M. Abdel-Fatah, 'Electrochemical frequency modulation and inductively coupled plasma atomic emission spectroscopy methods for monitoring corrosion rates and inhibition of low alloy steel corrosion in $\mathrm{HCl}$ solutions and a test for validity of the Tafel extrapolation method', Corros. Sci., vol. 51, no. 4, pp. 882894, Apr. 2009.

[37] M. A. Amin, S. S. A. E. Rehim, M. M. El-Naggar, and H. T. M. Abdel-Fatah, 'Assessment of EFM as a new nondestructive technique for monitoring the corrosion inhibition of low chromium alloy steel in $0.5 \mathrm{M} \mathrm{HCl}$ by tyrosine', J. Mater. Sci., vol. 44, no. 23, pp. 62586272, Dec. 2009.

[38] M. A. Amin, K. F. Khaled, Q. Mohsen, and H. A. Arida, 'A study of the inhibition of iron corrosion in $\mathrm{HCl}$ solutions by some amino acids', Corros. Sci., vol. 52, no. 5, pp. 1684-1695, May 2010.

[39] M. Bethencourt, F. J. Botana, J. J. Calvino, M. Marcos, and M. A. Rodríguez-Chacón, 'Lanthanide compounds as environmentally-friendly corrosion inhibitors of aluminium alloys: a review', Corros. Sci., vol. 40, no. 11, pp. 1803-1819, Nov. 1998.

[40] A. J. Aldykewicz, H. S. Isaacs, and A. J. Davenport, 'The Investigation of Cerium as a Cathodic Inhibitor for Aluminum-Copper Alloys', J. Electrochem. Soc., vol. 142, no. 10, pp. 3342-3350, Oct. 1995.

[41] A. J. Aldykiewicz, A. J. Davenport, and H. S. Isaacs, 'Studies of the Formation of CeriumRich Protective Films Using X-Ray Absorption Near-Edge Spectroscopy and Rotating Disk Electrode Methods', J. Electrochem. Soc., vol. 143, no. 1, pp. 147-154, Jan. 1996.

[42] M. A. Arenas, A. Conde, and J. J. de Damborenea, 'Cerium: a suitable green corrosion inhibitor for tinplate', Corros. Sci., vol. 44, no. 3, pp. 511-520, Mar. 2002.

[43] M. A. Arenas and J. de Damborenea, 'Interference by cerium cations during the multi-step zinc dissolution process in a chloride-containing electrolyte', Corros. Sci., vol. 48, no. 10, pp. 3196-3207, Oct. 2006.

[44] M. F. Montemor, A. M. Simões, and M. G. S. Ferreira, 'Composition and behaviour of cerium films on galvanised steel', Prog. Org. Coat., vol. 43, no. 4, pp. 274-281, Dec. 2001.

[45] M. A. Arenas and J. J. de Damborenea, 'Growth mechanisms of cerium layers on galvanised steel', Electrochimica Acta, vol. 48, no. 24, pp. 3693-3698, Oct. 2003.

[46] M. A. Arenas and J. J. de Damborenea, 'Surface characterisation of cerium layers on galvanised steel', Surf. Coat. Technol., vol. 187, no. 2-3, pp. 320-325, Oct. 2004.

[47] N. C. Hosking, M. A. Ström, P. H. Shipway, and C. D. Rudd, 'Corrosion resistance of zincmagnesium coated steel', Corros. Sci., vol. 49, no. 9, pp. 3669-3695, Sep. 2007.

[48] D. Christian, 'Performance of Prepainted and Unpainted Galfan Test Panels in Corrosive Environments', in Proc. 8th Galfan Licencees Meeting, held April, 1986, vol. 21.

[49] J. Elvins, J. A. Spittle, and D. A. Worsley, 'Microstructural changes in zinc aluminium alloy galvanising as a function of processing parameters and their influence on corrosion', Corros. Sci., vol. 47, no. 11, pp. 2740-2759, Nov. 2005.

[50] F. Hinterberger, W. Maschek, and J. Faderl, 'Influence of Typical Process Parameters on the Microstructure of Galfan Coatings', in Zinc-Based Steel Coating Systems: Production and Performance, The Minerals, Metals \& Materials Society, 1998, pp. 281-292.

[51] F. E. Goodwin and R. N. Wright, 'The Process Metallurgy of Zinc-Coated Steel Wire and Galfan ${ }^{\circledR}$ Bath Management', Apr. 2017.

[52] J. Elvins, J. A. Spittle, J. H. Sullivan, and D. A. Worsley, 'The effect of magnesium additions on the microstructure and cut edge corrosion resistance of zinc aluminium alloy galvanised steel', Corros. Sci., vol. 50, no. 6, pp. 1650-1658, Jun. 2008.

[53] H. N. McMurray, 'Localized Corrosion Behavior in Aluminum-Zinc Alloy Coatings Investigated Using the Scanning Reference Electrode Technique', Corrosion, vol. 57, no. 4, pp. 313-322, Apr. 2001. 
[54] Y. H. Zhu, H. C. Man, and W. B. Lee, 'Exothermic reaction in eutectoid Zn-Al based alloys', Mater. Sci. Eng. A, vol. 268, no. 1, pp. 147-153, Aug. 1999.

[55] J. Peng, L. Zhong, Y. Wang, Y. Lu, and F. Pan, 'Effect of extrusion temperature on the microstructure and thermal conductivity of $\mathrm{Mg}-2.0 \mathrm{Zn}-1.0 \mathrm{Mn}-0.2 \mathrm{Ce}$ alloys', Mater. Des., vol. 87, pp. 914-919, Dec. 2015.

[56] S. Bannour, K. Abderrazak, S. Mattei, J. E. Masse, M. Autric, and H. Mhiri, 'The influence of position in overlap joints of $\mathrm{Mg}$ and $\mathrm{Al}$ alloys on microstructure and hardness of laser welds', J. Laser Appl., vol. 25, no. 3, p. 032001, Feb. 2013.

[57] A. R. Marder, 'The metallurgy of zinc-coated steel', Prog. Mater. Sci., vol. 45, no. 3, pp. 191-271, Jun. 2000.

[58] M. S. I. T. Msit ${ }^{\circledR}$, 'Al-Mg-Zn (Aluminium - Magnesium - Zinc)', in Light Metal Systems. Part 3, Springer, Berlin, Heidelberg, pp. 1-19.

[59] S. Schuerz et al., 'Corrosion behaviour of $\mathrm{Zn}-\mathrm{Al}-\mathrm{Mg}$ coated steel sheet in sodium chloridecontaining environment', Corros. Sci., vol. 51, no. 10, pp. 2355-2363, Oct. 2009.

[60] A. Dong et al., 'Effect of Mg on the Microstructure and Corrosion Resistance of the Continuously Hot-Dip Galvanizing Zn-Mg Coating', Materials, vol. 10, no. 8, p. 980, Aug. 2017.

[61] T. Prosek, N. Larché, M. Vlot, F. Goodwin, and D. Thierry, 'Corrosion performance of ZnAl-Mg coatings in open and confined zones in conditions simulating automotive applications', Mater. Corros., vol. 61, no. 5, pp. 412-420, 2010.

[62] Y. Morimoto et al., 'Excellent Corrosion-resistant Zn-Al-Mg-Si Alloy Hot-dip Galvanized Steel Sheet" SUPER DYMA"', Apr. 2017.

[63] A. Komatsu, T. Tsujimura, K. Watanabe, N. Yamaki, A. Andoh, and T. Kittaka, 'Hot-dip Zn$\mathrm{Al}-\mathrm{Mg}$ coated steel sheet excellent in corrosion resistance and surface appearance and process for the production thereof', US6235410 B1, 22-May-2001.

[64] R. Bleeker and F. Hannour, 'Corus' new hot dip ZnAlMg coated steel for automotive applications', in Galvatech, 2007, vol. 7.

[65] P. Volovitch, C. Allely, and K. Ogle, 'Understanding corrosion via corrosion product characterization: I. Case study of the role of $\mathrm{Mg}$ alloying in $\mathrm{Zn}-\mathrm{Mg}$ coating on steel', Corros. Sci., vol. 51, no. 6, pp. 1251-1262, Jun. 2009.

[66] P. Volovitch, T. N. Vu, C. Allély, A. Abdel Aal, and K. Ogle, 'Understanding corrosion via corrosion product characterization: II. Role of alloying elements in improving the corrosion resistance of Zn-Al-Mg coatings on steel', Corros. Sci., vol. 53, no. 8, pp. 2437-2445, Aug. 2011.

[67] S. Schuerz et al., 'Corrosion behaviour of $\mathrm{Zn}-\mathrm{Al}-\mathrm{Mg}$ coated steel sheet in sodium chloridecontaining environment', Corros. Sci., vol. 51, no. 10, pp. 2355-2363, Oct. 2009.

[68] S. Schürz et al., 'Chemistry of corrosion products on $\mathrm{Zn}-\mathrm{Al}-\mathrm{Mg}$ alloy coated steel', Corros. Sci., vol. 52, no. 10, pp. 3271-3279, Oct. 2010.

[69] T. Koll, K. Ullrich, J. Faderl, J. Hagler, B. Schuhmacher, and A. Spalek, 'Properties and potential applications of novel ZnMg alloy coatings on steel sheet', Rev. Métallurgie, vol. 101, no. 7-8, pp. 543-550, Sep. 2004.

[70] N. LeBozec et al., 'Corrosion performance of Zn-Mg-Al coated steel in accelerated corrosion tests used in the automotive industry and field exposures', Mater. Corros., 2013.

[71] T. Prosek, A. Nazarov, U. Bexell, D. Thierry, and J. Serak, 'Corrosion mechanism of model zinc-magnesium alloys in atmospheric conditions', Corros. Sci., vol. 50, no. 8, pp. 22162231, Aug. 2008.

[72] T. Prosek, D. Persson, J. Stoulil, and D. Thierry, 'Composition of corrosion products formed on $\mathrm{Zn}-\mathrm{Mg}$, Zn-Al and $\mathrm{Zn}-\mathrm{Al}-\mathrm{Mg}$ coatings in model atmospheric conditions', Corros. Sci., vol. 86, pp. 231-238, Sep. 2014. 
[73] D. Persson, D. Thierry, N. LeBozec, and T. Prosek, 'In situ infrared reflection spectroscopy studies of the initial atmospheric corrosion of Zn-Al-Mg coated steel', Corros. Sci., vol. 72, pp. 54-63, Jul. 2013.

[74] R. Hausbrand, M. Stratmann, and M. Rohwerder, 'Corrosion of zinc-magnesium coatings: Mechanism of paint delamination', Corros. Sci., vol. 51, no. 9, pp. 2107-2114, Sep. 2009.

[75] T. Prosek et al., 'Effect of the microstructure of Zn-Al and Zn-Al-Mg model alloys on corrosion stability', Corros. Sci., vol. 110, pp. 71-81, Sep. 2016.

[76] M. Salgueiro Azevedo, C. Allély, K. Ogle, and P. Volovitch, 'Corrosion mechanisms of $\mathrm{Zn}(\mathrm{Mg}, \mathrm{Al})$ coated steel in accelerated tests and natural exposure: 1 . The role of electrolyte composition in the nature of corrosion products and relative corrosion rate', Corros. Sci., vol. 90, pp. 472-481, Jan. 2015.

[77] M. Vlot, M. Zuijderwijk, M. Toose, L. Elliot, R. Bleeker, and T. Maalman, 'Hot dip ZnAIMg coatings: microstructure and forming properties', Proc. Galvatech '07 Osaka Jpn., 2007.

[78] J. Sullivan, S. Mehraban, and J. Elvins, 'In situ monitoring of the microstructural corrosion mechanisms of zinc-magnesium-aluminium alloys using time lapse microscopy', Corros. Sci., vol. 53, no. 6, pp. 2208-2215, Jun. 2011.

[79] J. Sullivan, N. Cooze, C. Gallagher, T. Lewis, T. Prosek, and D. Thierry, 'In situ monitoring of corrosion mechanisms and phosphate inhibitor surface deposition during corrosion of zinc-magnesium-aluminium (ZMA) alloys using novel time-lapse microscopy', Faraday Discuss., vol. 180, no. 0, pp. 361-379, Jul. 2015.

[80] D. A. Worsley, D. Williams, and J. S. G. Ling, 'Mechanistic changes in cut-edge corrosion induced by variation of organic coating porosity', Corros. Sci., vol. 43, no. 12, pp. 23352348, Dec. 2001.

[81] S. Böhm, H. N. McMurray, S. M. Powell, and D. A. Worsley, 'Photoelectrochemical investigation of corrosion using scanning electrochemical techniques', Electrochimica Acta, vol. 45, no. 14, pp. 2165-2174, Mar. 2000.

[82] D. J. Penney, J. H. Sullivan, and D. A. Worsley, 'Investigation into the effects of metallic coating thickness on the corrosion properties of $\mathrm{Zn}-\mathrm{Al}$ alloy galvanising coatings', Corros. Sci., vol. 49, no. 3, pp. 1321-1339, Mar. 2007.

[83] J. Sullivan, C. Weirman, J. Kennedy, and D. Penney, 'Influence of steel gauge on the microstructure and corrosion performance of zinc alloy coated steels', Corros. Sci., vol. 52, no. 5, pp. 1853-1862, May 2010.

[84] A. M. Simões and J. C. S. Fernandes, 'Studying phosphate corrosion inhibition at the cut edge of coil coated galvanized steel using the SVET and EIS', Prog. Org. Coat., vol. 69, no. 2, pp. 219-224, Oct. 2010.

[85] D. A. Worsley, H. N. McMurray, J. H. Sullivan, and I. P. Williams, 'Quantitative Assessment of Localized Corrosion Occurring on Galvanized Steel Samples Using the Scanning Vibrating Electrode Technique', Corrosion, vol. 60, no. 5, pp. 437-447, May 2004.

[86] H. N. McMurray and D. A. Worsley, in Research in Chemical Kinetics Volume IV, 1 edition., vol. IV, R. Compton and G. Hancock, Eds. Amsterdam; New York: Wiley-Blackwell, 1997, p. 256.

[87] H.-K. Sohn and J.-W. Lee, 'Corrosion Behaviors of Zn-MgZn2 eutectic structure in Zn-Al-Mg coatings', in Gal-vatech, II, 8th International Conference on Zinc and Zinc Al-loy Coated Steel Sheet [Cj. Genova: IZA, 2011. 144.

[88] H. N. McMurray, G. Parry, and B. D. Jeffs, 'Corrosion resistance of Zn-Al alloy coated steels investigated using electrochemical impedance spectroscopy', Ironmak. Steelmak. Lond., vol. 25, no. 3, p. 210, 1998.

[89] H. Dafydd, D. A. Worsley, and H. N. McMurray, 'The kinetics and mechanism of cathodic oxygen reduction on zinc and zinc-aluminium alloy galvanized coatings', Corros. Sci., vol. 47, no. 12, pp. 3006-3018, Dec. 2005. 
[90] R. Hausbrand, M. Stratmann, and M. Rohwerder, 'The Physical Meaning of Electrode Potentials at Metal Surfaces and Polymer/Metal Interfaces: Consequences for Delamination', J. Electrochem. Soc., vol. 155, no. 7, pp. C369-C379, Jul. 2008.

[91] C. Yao, Z. Wang, S. L. Tay, T. Zhu, and W. Gao, 'Effects of Mg on microstructure and corrosion properties of Zn-Mg alloy', J. Alloys Compd., vol. 602, pp. 101-107, Jul. 2014.

[92] T. Falk, J.-E. Svensson, and L.-G. Johansson, 'The Influence of $\mathrm{CO} 2$ and $\mathrm{NaCl}$ on the Atmospheric Corrosion of Zinc A Laboratory Study', J. Electrochem. Soc., vol. 145, no. 9, pp. 2993-2999, Sep. 1998.

[93] D. Thierry, T. Prosek, N. LeBozec, and E. Diler, 'Corrosion protection and corrosion mechanisms of continuous galvanised steel sheet with focus on new coating alloys', Proc. Galvatech'11 Int. Conf. Zinc Zinc Alloy Coat. Steel, Jun. 2011.

[94] F. L. Floyd, S. Tatti, and T. Provder, 'Using DC electrochemical techniques to assess the relative corrosiveness of water-based coatings and their ingredients', J. Coat. Technol. Res., vol. 4, no. 2, pp. 111-129, Jun. 2007.

[95] M. Salgueiro Azevedo, C. Allély, K. Ogle, and P. Volovitch, 'Corrosion mechanisms of $\mathrm{Zn}(\mathrm{Mg}, \mathrm{Al})$ coated steel: 2 . The effect of $\mathrm{Mg}$ and Al alloying on the formation and properties of corrosion products in different electrolytes', Corros. Sci., vol. 90, pp. 482490, Jan. 2015.

[96] E. McCafferty, 'Validation of corrosion rates measured by the Tafel extrapolation method', Corros. Sci., vol. 47, no. 12, pp. 3202-3215, Dec. 2005.

[97] T. S. Narayanan, 'Surface pretreatment by phosphate conversion coatings - a review', Rev Adv Mater Sci, vol. 9, no. 2, pp. 130-177, 2005.

[98] W.-H. Kok, 'Formation of zinc phosphate coatings on aluminum alloys', University of British Columbia, 2001.

[99] D. B. Freeman, 'Phosphating and metal pre-treatment', B Freeman Woodhead-Faulkner Camb. 1986 229, p. 30, 1986.

[100] K. Ravichandran and T. S. Narayanan, 'Studies on acceleration of the low temperature zinc phosphating processes', Trans. IMF, vol. 79, no. 4, pp. 143-145, 2001.

[101] T. S. N. Sankara Narayanan and M. Subbaiyan, 'Overheating-Its decisive role in phosphating', Met. Finish., vol. 93, no. 1, pp. 30-31, Jan. 1995.

[102] G. Williams, H. N. McMurray, and R. Grace, 'Inhibition of magnesium localised corrosion in chloride containing electrolyte', Electrochimica Acta, vol. 55, no. 27, pp. 7824-7833, Nov. 2010.

[103] X. Zhou et al., 'Synthesis of zinc phosphate and zinc ammonium phosphate nanostructures with different morphologies through pH control', Mater. Charact., vol. 108, pp. 22-28, Oct. 2015.

[104] M. Mobin, S. Zehra, and M. Parveen, 'I-Cysteine as corrosion inhibitor for mild steel in $1 \mathrm{M}$ $\mathrm{HCl}$ and synergistic effect of anionic, cationic and non-ionic surfactants', J. Mol. Liq., vol. 216, pp. 598-607, Apr. 2016.

[105] K. F. Khaled, 'Corrosion control of copper in nitric acid solutions using some amino acids A combined experimental and theoretical study', Corros. Sci., vol. 52, no. 10, pp. 32253234, Oct. 2010.

[106] H. Ashassi-Sorkhabi, Z. Ghasemi, and D. Seifzadeh, 'The inhibition effect of some amino acids towards the corrosion of aluminum in $1 \mathrm{M} \mathrm{HCl}+1 \mathrm{M} \mathrm{H} 2 \mathrm{SO} 4$ solution', Appl. Surf. Sci., vol. 249, no. 1, pp. 408-418, Aug. 2005.

[107] H. Nady, 'Tricine [N-(Tri(hydroxymethyl)methyl)glycine] - A novel green inhibitor for the corrosion inhibition of zinc in neutral aerated sodium chloride solution', Egypt. J. Pet., Feb. 2016.

[108] G. Moretti and F. Guidi, 'Tryptophan as copper corrosion inhibitor in $0.5 \mathrm{M}$ aerated sulfuric acid', Corros. Sci., vol. 44, no. 9, pp. 1995-2011, Sep. 2002. 
[109] J.-J. Fu, S.-N. Li, L.-H. Cao, Y. Wang, L.-H. Yan, and L.-D. Lu, 'I-Tryptophan as green corrosion inhibitor for low carbon steel in hydrochloric acid solution', J. Mater. Sci., vol. 45, no. 4, pp. 979-986, Feb. 2010.

[110] R. C. Armstrong and A. J. Swallow, 'Pulse- and Gamma-Radiolysis of Aqueous Solutions of Tryptophan', Radiat. Res., vol. 40, no. 3, pp. 563-579, Dec. 1969.

[111] N. Wint, J. H. Sullivan, and D. J. Penney, 'The Role of pH on the Inhibition of Aqueous Zinc Corrosion by L-tryptophan', J. Electrochem. Soc., vol. 164, no. 7, pp. C356-C366, Jan. 2017.

[112] B. Dolińska, 'The properties of solid Zn(II)-amino acid complexes in the form of suspensions', Il Farm., vol. 56, no. 10, pp. 737-740, Sep. 2001.

[113] J. Duchoslav et al., 'Nanoscopic view on the initial stages of corrosion of hot dip galvanized Zn-Mg-Al coatings', Corros. Sci., vol. 83, pp. 327-334, Jun. 2014. 


\section{Appendix}

Appendix 1

As stipulated in the relevant chapters, some of the work completed has gone to journal

publication. Consequently, this can be found via the following reference:

J. Sullivan, N. Cooze, C. Gallagher, T. Lewis, T. Prosek, and D. Thierry, 'In situ monitoring of corrosion mechanisms and phosphate inhibitor surface deposition during corrosion of zincmagnesium-aluminium (ZMA) alloys using novel time-lapse microscopy', Faraday Discuss., vol. 180, no. 0, pp. 361-379, Jul. 2015. 